\title{
INFLUENCE OF TOPOGRAPHY ON THE DYNAMICS OF BAROCLINIC OCEANIC EDDIES
}

by

\section{Kirill Konstantinovich Pankratov}

M.S. Moscow Institute of Physics and Technology, 1988.

Doctor of Philosophy

Massachusetts Institute of Technology

and

MARINE

BHOLOGICAL

Submitted in partial fulfillment of the requirements for the degree of

LABORATORY

Woods Hole Oceanographic Institution

LIBRARY

WOBS HOLE, KASE.

พ.. 11.0 .1 .

February 1994

(C) Kirill Pankratov, 1994

The author hereby grants to MIT and to WHOI permission to reproduce and to distribute copies of this thesis document in whole or in part.

Certified by

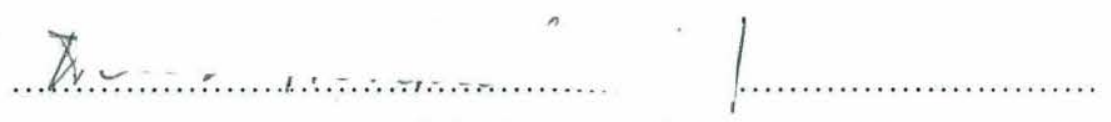

Joint Program in Physical Oceanography

Massachusetts Institute of Technology

Woods Hole Oceanographic Institution

Signature of Author

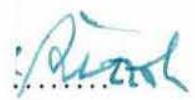

Paola Malanotte-Rizzoli

Thesis Advisor

Accepted by

Lawrence J. Pratt

Chairman ${ }_{6}=$ for $_{0}$ mittee for Physical Oceanography

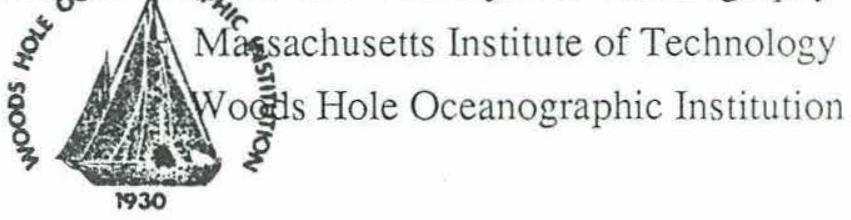




\title{
INFLUENCE OF TOPOGRAPHY ON THE DYNAMICS OF BAROCLINIC OCEANIC EDDIES by \\ Kirill Pankratov
}

\begin{abstract}
Submitted in partial fulfillment of the requirements for the degree of Doctor of Philosophy at the Massachusetts Institute of Technology - Woods Hole Oceanographic Institution
\end{abstract}

February 1994.

\begin{abstract}
In this work we study motion of a baroclinic upper-ocean eddy over a large-scale topography which simulates a continental slope. We use a quasigeostrophic f-plane approximation with continuous stratification. To study this problem we develop a new numerical technique which we call "semi-lagrangian contour dynamics". This technique resembles the traditional 2-D contour dynamics method but differs significantly from it in the numerical algorithm. In addition to "Lagrangian" moving contours it includes an underlying "Eulerian" regular grid to which vorticity or density fields are interpolated. To study topographic interactions in a continuously stratified model we use density contours at the bottom in a similar manner as vorticity contours are used in the standard contour dynamics. For the case of a localized upper-ocean vortex moving over a sloping bottom the problem becomes computationally 2-dimensional (we need to follow only bottom density contours and the position of the vortex itself) although the physical domain is still 3-dimensional.

Results of the numerical model indicate importance of baroclinic effects in the vortextopography interaction. After the initial surge of topographic Rossby waves a vortex moves almost steadily due to the interaction with a bottom density anomaly which is created and supported by a vortex itself. This anomaly is equivalent to a region of opposite-signed vorticity with a total circulation exactly compensating that of a vortex. This results in a vertically aligned dipolar structure with the total barotropic component equal to zero. Analytical considerations explaining this effect are presented and formulated in a more general statement which resembles but does not coincide with the "zero angular momentum theorem" of Flierl, Stern and Whitehead, 1983.

In such steady translation the centroid of a bottom density anomaly is displaced horizontally from the center of an upper-ocean vortex so the whole system moves due to this misalignment, which is known as a "hetonic mechanism". Cyclonic vortices go generally upslope, and anticyclones - in a downslope direction. The along-slope component of their motion depends upon the strength of a vortex, curvature of the bottom slope and background flows. When surrounded by a bowl-shaped topography anticyclonic vortices tend to stay near the deepest center of a basin, even resisting ambient flows which advect them outward. Application of this results to various oceanic examples (particularly to the "Shikmona eddy" in the Eastern Mediterranian) is discussed.

Our results show that the behavior of a vortex over a sloping bottom differs significantly from its motion on the planetary beta-plane (but with a flat bottom). To explain this difference we introduce the concept of a "wave-breaking regime" relevant for the case of a planetary beta-effect, and a "wave-gliding regime" which characterizes the interaction of an eddy with a topographic slope.
\end{abstract}




\section{Acknowledgements}

In preparation of this document I would like to extend my appreciation and gratitude to many people who helped me during the work. First of all I would like to mention the following persons:

My adviser - Paola Rizzoli who encouraged me to do this work and constantly provided scientific, moral (and material) support in the course of it.

All other members of my comittee - David Chapman, Glenn Flierl, Joe Pedlosky and Larry Pratt, who followed this work attentively and occasionally gave me tough times during our meetings (which helped a lot to improve this thesis). They also added hundreds of useful suggestions and corrections to this document.

Especially I want to thank Glenn Flierl for many ideas and insight into the problem and his constant interest and help in the course of this work.

Numerous scientists at MIT and WHOI with whom I discussed the ideas and results of this study.

I also had an opportunity to present various parts of this work in many other places, during the conferences and occasional visits. Among the numerous scientists with whom I had productive discussions on the related topics I would like to mention Steve Meacham, Melvin Stern, Benoit Cushman-Roisin from various institutions here in United States, and Vladimir Zhmur, George Sutyrin, Vladimir Kamenkovich from the Shirshov Institute of Oceanology in Moscow.

I would like also to thank warmly the Joint Program as a whole, all the people whose efforts make it (I have no doubts about this) the best graduate program in the world. In particular I am grateful to Abbie Jackson, who helped me to come here and during my first days in the United States, and to Mary-Jane Tucci who provides us (students) with such a nice accomodations in Woods Hole where I had a wonderful time with my wife and friends during the summers of my stay here.

This work was supported by the NSF grant \# OCE 90-12821. 


\section{Contents}

Abstract $\quad$.......................................................................... 2

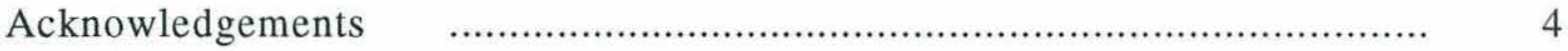

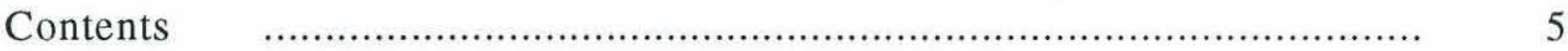

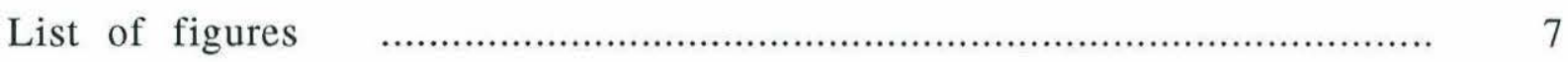

\section{Introduction}

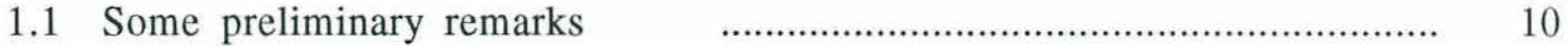

1.2 Observations $\quad$......................................................................... 14

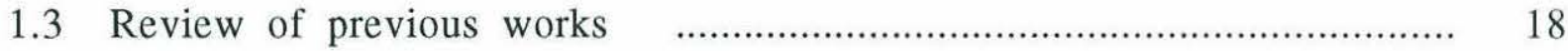

\section{Mesoscale flows over topography:} some theoretical considerations

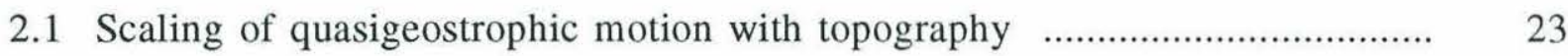

2.2 The bottom boundary condition …................................................. 30

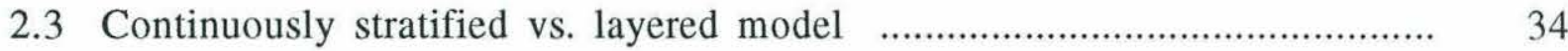

\section{Steady motion of a baroclinic point vortex} along a sloping bottom

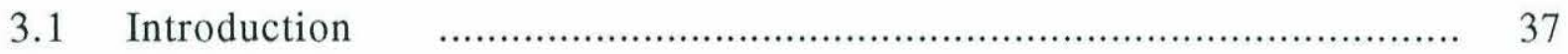

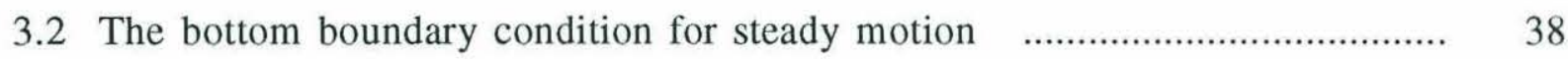

3.3 Formulation of the problem for a steadily translating point vortex .................... 41

3.4 Solution for the limiting cases f..................................................... 45

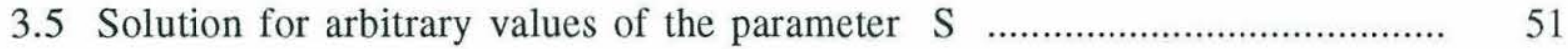

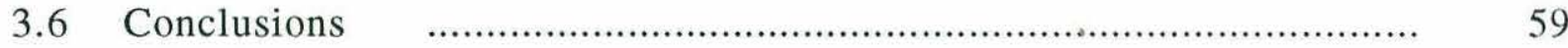

\section{Semi-lagrangian contour dynamics}

for a continuously stratified ocean

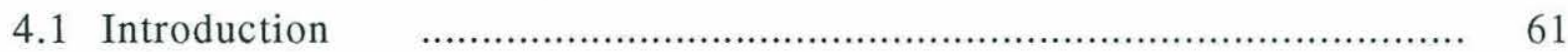

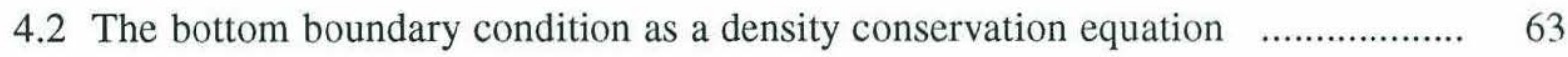

4.3 Formulation of the problem …........................................................... 66

4.4 Green's function for PV and density anomalies for the flat bottom case $\quad . . . . . . . . . . . \quad 68$

4.5 Green's function for a variable bottom …............................................ 71 
5 Interaction of a baroclinic vortex with a sloping bottom

5.1 Model setup and initial conditions ...................................................... 80

5.2 Scaling and nondimensionalization ……….......................................... 86

5.3 Initial evolution of a vortex …….................................................... 89

5.4 Later stage: steady motion …....................................................... 93

5.5 "Hetonic" translation mechanism ………............................................. 103

5.6 "No barotropic component" theorem ………....................................... 106

6 Different factors influencing vortex-topography interaction

6.1 Curvature of the bottom and topographic irregularities .............................. 113

6.2 Presence of background flows ………......................................... 125

6.4 Non-uniform ambient stratification ……........................................ 134

6.5 Finite-volume vortex ….......................................................... 135

7 Planetary vs. topographic beta-effect

7.1 Initial evolution: similarities ………................................................. 140

7.2 Differences: "wave-gliding" vs. wave-breaking" regimes ……..................... 142

7.3 Comparison of a vortex representation in a continuously stratified and 2-layer model

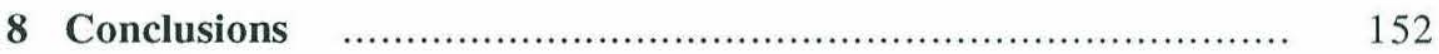

Appendix 1 Parameterization of variable buoyancy frequency

A 1.1 Introduction ...................................................................... 156

A1.2 "Parametric" buoyancy frequency profiles ……………....................... 158

A1.3 Vertical structure and dispersion relationship for topographic and planetary Rossby waves ……........................................................ 165

A1.4 Green's function and azimuthal velocity for the case of "parametric" variable stratification

Appendix 2 Some details of the numerical implementation of the semilagrangian contour dynamics method ….................... 180

References 


\section{List of figures}

$1.1(\mathrm{a}, \mathrm{b}, \mathrm{c})$. Observations of vortices over continental slopes.

1.2. Observed positions of the "Shikmona eddy" in the Levantine basin.

1.3. Schematic propagation tendency for planetary and topographic $\beta$.

3.1 A baroclinic vortex above a sloping bottom.

3.2 Coordinate systems (x,y,z) and (x,y, $\left.\mathrm{z}^{\prime}\right)$ used for the solution.

$3.3(a, b)$ Real and image vortices for limiting values of the parameter $S$.

3.4 (a,b) The system of images when surface is present.

3.5 Dependence of parameter $v$ on the angle $\alpha$.

3.6(a,b) (a) Dependence of $v$ on $S$ for a semi-infinite ocean

(b) Dependence of $v$ on the non-dimensional strength $\mathrm{A}$ of a vortex

3.7 Numerator B versus $\mathrm{S}$ at the second-order pole in the equation (3.28)

$3.8 \quad$ (a) Dependence of $v$ on $S$ in the presence of a surface.

(b) Dependence of $v$ on the non-dimensional strength A of a vortex.

4.1 Stratified ocean with bottom topography.

4.2 System of images for a flat bottom.

4.3 Lagrangian moving contours and Eulerian regular grid.

$5.1(a, b) \quad$ (a) Top view of the domain with a vortex.

(b) Side view of the domain configuration.

$5.2(a, b) \quad$ (a) Mesh plot of contour displacements in the wave dispersion test.

(b) Evolution of the middle contour in the test run.

5.3 Typical "sponge matrix" for displacements of density contours.

$5.4 \quad$ Velocity and angular velocity from a surface point vortex.

5.5 Velocities at the bottom with unperturbed density contours.

5.6 Initial dipolar structure in the vorticity field at the bottom.

5.7 (a,b) Displacement of density contours and velocity field at the early stage.

$5.8(\mathrm{a}, \mathrm{b})$ The vortex trajectory and a bottom density anomaly for a cyclonic vortex with $\mathrm{A}=.4$

5.9 (a,b) Same as 5.8 but for $\mathrm{A}=1$.

5.10 (a,b) Same as 5.8 but for $A=1.5$.

$5.11(\mathrm{a}, \mathrm{b})$ The vortex trajectory and a bottom density anomaly for a cyclonic vortex with $\mathrm{A}=.6$ 
and the bottom boundary condition at the actual depth.

5.12 (a,b) Same as 5.11 but the bottom boundary condition at the reference depth.

5.13 Trajectories of identical-twin runs with a fixed grid with bottom boundary condition at the reference level and at the actual depth.

5.14 Velocity field at the bottom for a steadily translating state.

5.15 Evolution of a total circulation from a bottom density anomaly, normalized by the vortex circulation.

5.16 "Hetonic efficiency parameter" vs. the amplitude A for different runs.

$5.17(a, b) \quad$ (a) Sketch of a steadily moving perturbation.

(b) Displacement of tracer isolines.

$5.18(\mathrm{a}, \mathrm{b})$ Streamfunction from a perturbation with a zero and non-zero net barotropic component

$6.1(\mathrm{a}, \mathrm{b})$ Motion of a cyclonic vortex over a slope with a positive curvature.

6.2 (a,b) Motion of a cyclonic vortex over a slope with a negative curvature.

6.3 (a,b) Motion of a cyclone (a) and anticyclone (b) in a fixed grid domain.

$6.4(\mathrm{a}, \mathrm{b})$ Motion of a cyclonic vortex over a "parabolic hill".

6.5 (a,b) Motion of an anticyclonic vortex over a "parabolic valley".

$6.6(a, b)$ Sketch of directions of vortex motion for different types of topography.

6.7 (a,b) Motion of a cyclonic vortex over a slope with irregularities.

$6.8(\mathrm{a}, \mathrm{b})$ Motion of an anticyclonic vortex over a slope with a "canyon".

$6.9(\mathrm{a}, \mathrm{b})$ Motion of a deep cyclonic vortex.

$6.10(a, b)$ Motion of a deep cyclone with a background "northward" flow, $v_{\text {flow }}=5$.

$6.11(\mathrm{a}, \mathrm{b})$ Motion of a deep cyclone with a background "southward" flow, $\mathrm{v}_{\text {flow }}=-5$.

6.12 (a,b) Motion of a deep cyclone with a background "southward" flow, $v_{\text {flow }}=-10$.

6.13 (a,b) Motion of a deep cyclone with a background "southward" flow, $v_{\text {flow }}=-5$ over a slope with a positive curvature.

$6.14(\mathrm{a}, \mathrm{b})$ Motion of a deep cyclone with a background "southward" flow, $\mathrm{v}_{\text {flow }}=-5$ over a slope with a negative curvature.

6.15 (a,b) Motion of a cyclonic vortex over a uniform slope and with a "parametric" stratification profile $\mathrm{N}_{\text {surface }} / \mathrm{N}_{\text {bottom }}=20$.

$6.16(a, b)$ Motion of a finite-volume vortex with a radius $R=2 R_{\text {def }}$ over a uniform slope.

7.1 Near-steady vortex motion on the planetary $\beta$-plane: the "wave-breaking" regime.

7.2 Steady vortex motion over a uniform slope: the "wave-gliding" regime.

7.3 Sketch of a vortex for a two-layer and a continuously stratified model. 
$7.4(\mathrm{a}, \mathrm{b}) \quad$ Velocities and angular velocities at the bottom from the vortex for a two-layer and a continuously stratified model.

A1.1 (a,b) Examples of "parametric" stratification profiles.

A1.2 Parameter plane $\left(\mathrm{m}_{0}, \tau\right)$.

A1.3 (a,b) Quasigeostrophic vertical modes for "parametric profiles".

A1.4 (a,b) Dispersion relation and vertical structure for topographic Rossby waves.

A1.5 (a,b) Examples of azimuthal velocity profiles from a point vortex.

A2.1 Evolution of the short waves packet.

A2.2 Comparison of theoretical and computed group velocities of short waves.

A2.3 Evolution of the dipolar perturbation. 


\section{Chapter 1}

\section{Introduction}

\subsection{Some preliminary remarks}

Bottom topography, as one of the factors influencing oceanic circulation, has probably received less attention in oceanographic literature (relative to its importance). One can think of the several reasons which could contribute to this situation.

Obvious importance of other factors like the wind stress and thermal forcing in driving the ocean circulation and apparent isolation of the deep ocean from these driving forces led to the widespread notion that deep waters are almost motionless and sluggish and this was one of the reasons to neglect deep flows and topographic influence in favor of other effects.

Introduction of realistic topography in a homogeneous ocean model produces unrealistically large torques acting upon water parcels due to their cross-isobath motion in the geostrophic dynamics. The resulting circulation looks much less like the observed one than in the case of a flatbottom ocean, because actual contours of $\mathrm{f} / \mathrm{H}$ are shifted too strongly from latitudinal circles. This introduced oceanographers to the idea that stratification must reduce effects of topography. It should happen because deep isopycnal surfaces can be displaced in such a manner as to diminish upperocean pressure anomalies and a complete compensation is achieved somewhere in the bulk of the ocean (Veronis, 1981). If topography does not protrude above this level then deeper waters are dynamically isolated and will not influence upper-ocean flows.

Certain observational considerations probably also contributed to this line of reasoning: oceanographers enjoyed relative abundance of hydrographic data in comparison with expensive direct current measurements, especially in deep waters. This urged them to try to extract velocities just from a density field using the "thermal wind" relation and presumptions about the "reference level" or "level of no motion" where the velocity goes to zero. It was usually presumed that this level should be somewhere at the large depths, for example near the bottom. This search for the "level of no motion" was a Quest for the Holy Grail, although not very successful one, for several generations

of oceanographers. Gradually increasing amount of direct measurements showed that the deep ocean is far from motionless and instead a dynamically active and changing medium. 
One can certainly imagine some (dissipative) mechanism of the adjustment of deep isopycnal surfaces to compensate for upper-ocean pressure anomalies in a slow steady circulation. But for time-dependent large-scale and especially for strong and turbulent mesoscale currents it is hardly possible: simple scaling arguments suggest that the influence of a surface flow should be felt throughout the whole water column for scales larger than the deformation radius. Although stratification can evidently reduce the coupling between the upper ocean and a bottom flow, this is unlikely to occur in such a simple manner as to make abyssal waters completely stagnant. The role of stratification also requires a more detailed analysis.

In this work we attempt to contribute to one particular area of this problem, namely to the issue of interaction of upper-ocean eddies with large-scale topography.

A rather general question that we are going to address can be formulated as following:

What effects on mesoscale dynamics can be produced by a combination of baroclinicity (stratification) and topographic variations (like a sloping bottom ) ?

Generally one can consider "purely barotropic" and surface-confined "equivalent barotropic" cases as two extreme views of the role of bottom topography. The first one - a homogeneous ocean model in which a total depth is included in the potential vorticity conservation for a fluid parcel and which presumably exaggerates the topographic influence. An opposite extreme is a class of reducedgravity models (the passive, infinitely deep lower layer approximation) where topography is shielded by stratification and completely ignored unless it protrudes into dynamically active layers. At a first glance one can suppose that the truth is somewhere in between these extreme cases for the realistically stratified ocean. But it can also happen that a correct combination of these two factors topography and stratification - can produce effects that are completely different from "purely barotropic " and "equivalent barotropic" models, or an unusual combination of some of their properties.

Before discussing some specific questions which we address in the present work we would like to accentuate several points in an attempt to explain and justify our approach.

In this study we would especially like to explore baroclinic effects, a combination of topography and stratification, for several reasons. Barotropic flows are better studied and easier to understand in terms of a simple vorticity - stretching balance. But observations show that the baroclinic mode is usually dominant for oceanic mesoscales, the more so in the presence of prominent topographic features. Topographic variations in the real ocean are large enough to act as very strong constraints for a barotropic flow. Yet, as we mentioned above, upper-ocean currents do not seem to be too constrained, although certainly influenced, by a variable relief. So stratification and baroclinicity play a major role. Moreover, topography in the presence of stratification provides an efficient mechanism for upper-ocean flows to adjust for various kinds of forcing. 
Another thing we would like to point out is that although a general problem which we have in mind is the interaction of mesoscales with topography, in this work we are going to concentrate on dynamics of an individual eddy. Yet we believe that study of a single eddy can substantially contribute to our understanding of a mesoscale ensemble over topography. There are several reasons why this can be so. Firstly, energetic mesoscale flows are dominated by strong localized vortices and it is this fully nonlinear regime we want to understand. Another reason is that the interaction of an eddy with a relief is most probably confined to a relatively quiet domain "just under" an eddy itself, while the influence of other factors like the beta-effect or an ambient shear are more distributed to surrounding turbulent waters. That is, the topographic interaction is probably more "individual" and "elementary" than other factors and more dependent on properties of a given eddy than on a whole ensemble. This approach to study mesoscales can be viewed as opposite to the purely statistical one operating in a wavenumber instead of a physical space. The presented arguments are rather questionable and speculative and we shall return to this issue below after discussing the results of the present study.

More specific questions that we are going to address in this work are following:

- In what direction and how fast a vortex can be forced to move by topographic forcing? Can topographic variations below influence its shape, strength and some internal dynamical processes significantly?

This is still a rather general question that one can ask about a vortex-topography interaction and we can hope to answer it here only partially, concerning some integral features, like motion of a whole vortex and far-field velocities.

- Does large-scale smooth topography produce effects similar to the planetary beta-effect? In a homogeneous ocean model the planetary beta-term is dynamically equivalent to a uniform bottom slope. But to what extent is the analogy between the planetary and the topographic beta-effect valid in a stratified ocean?

This question is particularly relevant to the eddy-topography interaction because the behavior of vortices was extensively studied on the planetary beta-plane and the simple analogy mentioned above is often invoked to explain or predict behavior of eddies over continental slopes.

- Is there a significant difference, symmetry or asymmetry, in the behavior of cyclonic and anticyclonic eddies due to bottom topography?

If so it can imply significant eddy momentum and heat fluxes induced by topography, anisotropic and "anomalous" diffusion effects. These fluxes can be important corrections to the momentum and heat balance of the ocean general circulation. Such effects apparently can not be parameterized in terms of simple eddy diffusion coefficients but rather can be determined from the geometry of ocean basins. 
- Which factors influence the eddy-topography interaction processes: a curvature and irregularities of topography, the strength and the size of an eddy, ambient flows, stratification, etc.? This question is interesting also in relation to the planetary beta-effect, which describes uniform variation of the coriolis parameter with a latitude. For a topographic case the situation is different: we do not have many uniform slopes of the oceanic bottom but instead often curved, ragged and irregular topographic features. Many numerical models dealt with simplified straight-line topography - strips of uniform slope or exponential depth variations. It is not quite clear how results of these models can hold for more realistic cases with convoluted isobaths. Here we do not however attempt to study very rough topographic features such as isolated seamounts. But even a rather smooth bathymetry can result in a different behavior than, for example, in a uniform slope case.

In this study we will use a continuously stratified model because in our view it is more capable of describing baroclinicity of a flow in a realistic ocean. This, as we shall demonstrate below, allows to avoid in our problem the vertical discretization and hence truncation of vertical degrees of freedom. We shall postpone now a detailed discussion and comparison of a continuous stratification and layered model until chapters 2 and 7.

This work is organized as following. After a brief review of some of the observational results and related theoretical and numerical works we consider in some details our assumptions and approach to this study. Then in chapter 2 we analyze the validity of the quasigeostrophic approximation for a flow over topography and compare a continuously stratified ocean model with a layered one. Since we work with a continuously stratified model we discuss some properties of stratification profiles (in Appendix 1). In particular we find a class of buoyancy frequency profiles which allow a simple analytical relationship between a streamfunction and a potential vorticity in a quasigeostrophic case similarly to a uniform stratification profile.

After that in chapter 3 we develop a semi-analytical model of motion of mesoscale vortex over a uniformly sloping bottom. In this model a vortex is allowed to move steadily along isobath, its velocity and direction of motion depend on its strength, the bottom slope, stratification and the depth of an ocean.

In chapter 4 we present the numerical model based on a new technique which we call "semilagrangian contour dynamics". It is with this model the main results of our study are obtained. This method is applied for 3-dimensional stratified problem. But in our case when a potential vorticity anomaly is localized in a single vortex the problem is reduced to a two-dimensional one without any truncation in the vertical, which is a very convenient simplification. Our technique uses positions of density contours at the bottom explicitly in a similar fashion as vorticity contours are used in the classical 2-D contour dynamics method.

In chapters 5 and 6 we use this method to study the initial value problem of evolution of a vortex over a sloping bottom. At the beginning density contours at the bottom are unperturbed. The 
velocity induced by a vortex displaces these contours which generates topographic Rossby waves. A vortex itself moves due to the interaction with a wave field. After a timescale of less than a characteristic period of topographic waves the whole system approaches a near-steady state. In this state the circulation induced by the bottom density anomaly compensates exactly that of the vortex so the total barotropic component vanishes. This appears to be a very robust result independent of the initial conditions and parameters of the problem. We present some analytical arguments explaining this effect and formulate a rather general statement resembling the "zero angular momentum" theorem of Flierl, Stern and Whitehead (1983).

The centroid of a bottom density anomaly is not completely aligned vertically with the center of a vortex so the resulting "hetonic" structure advects itself. Motion is predominantly cross-slope with cyclonic vortices going in the uphill direction and anticyclones - downhill. Accompanying alongisobath motion can be associated with some cross-slope asymmetries, like a bottom curvature or a background along-slope shear flow. It is interesting to note that the along-isobath component can be either to the right or to the left of the upslope direction in different situations. This contrasts to vortex motion on the planetary beta-plane where the westward translation is common for both cyclones and anticyclones. We present a qualitative explanation (in chapter 7) why this can be so.

Finally we present some conclusions and discuss possible relevance and significance of our results in a general context of mesoscales-topography interaction. We also consider limitations of our model and some suggestions for its improvement.

\subsection{Observations}

A majority of the energetic ocean eddies are produced in the regions not far from a coast - for example near western (and eastern) boundary currents. These areas typically have rather steep continental slopes as well as other topographic features such as seamounts, canyons etc. No wonder there are many evidences of the topographic influence on dynamics of these eddies. Yet it is usually difficult to distinguish between topography and other important factors. Because these eddies exist in a very turbulent environment it is not easy to extract the topographic influence in its "pure" form. The interpretation of observational results is made difficult due to the fact that most of the existing data are confined to the upper ocean without simultaneous hydrographic and velocity measurements near the bottom, where these topographic interactions can be inferred.

Two areas where motion of strong eddies near continental slopes were studied most extensively are the Gulf Stream - Sargasso Sea region and the western part of the Gulf of Mexico. 
In the Gulf Stream we have an abundance of observations of large cyclonic and anticyclonic rings detached from the stream itself as well as many smaller eddies of different types. Warm core rings tend to go northwest until reaching the continental slope. After that their behavior can vary but they often go to the southwest approximately along the depth contours until being reabsorbed into the Stream again.

The trajectory of the ring WCR-82B - one of the best observed warm-core rings - showed a remarkable coincidence with contours of 2500-3000 isobaths (see figure 1.1a) as it moved to the southwest along the continental slope with the velocity of $5-15 \mathrm{~cm} / \mathrm{s}$ (Evans et al, 1985). Several times its motion was apparently perturbed over irregularities in the bottom relief, like the Hudson canyon, which suggest a strong topographic influence. Still these rings do not always behave in such a simple way. For example, Cornillon et al (1989) analyzed trajectories of many warm core rings relative to the slope waters and found a large scatter in speeds and directions of their motion.

Observations of near-bottom velocities on the continental slope and rise north of the Gulf Stream show occasional bursts of topographic Rossby waves associated with the Gulf Stream meanders and passages of warm core rings (Kelley and Weatherly, 1985). This indicates at least the strong coupling between upper-ocean structures like rings and motion in deep waters. Yet such measurements are rare and spatially too isolated to infer the detailed characteristics of such interactions. The interpretation of these data is also complicated by the presence of relatively strong and unsteady near-bottom flows like the "cold filament" of Weatherly and Kelley (1982), associated with the deep western boundary current.

On the other side of the Gulf Stream - in the Sargasso Sea and the Blake Plateau region - there are many observations of strong cyclonic eddies like cold core rings. They often seem to move in a different way relative to a bottom relief than anticyclonic warm core rings. One localized subsurface cyclone was studied for several months during the POLYMODE experiment (Ivanov and Paramonov, 1980). Its trajectory followed $5000 \mathrm{~m}$ isobath quite accurately as it moved to the northwest - to the right of the upslope direction (figure 1.2b). Many cold core rings penetrate into rather shallow waters up to a depth of about 1000m (Cheney et al, 1976). This upslope and predominantly westward motion can be caused by the planetary beta-effect but topography can also play an important role.

Large anticyclonic rings of the Gulf of Mexico drift usually westward after detaching from the Loop current until reaching the steep continental slope of this area (Lewis and Kirwan, 1985). Then they usually move northward, their trajectories can be very curved, as if they tend to depart from the slope but then are pushed onto it again. These observations were made usually from surface drifters (see, for example, the drifter trajectory in figure 1.1c) and simultaneous measurements of a nearbottom flow structure are not available. 

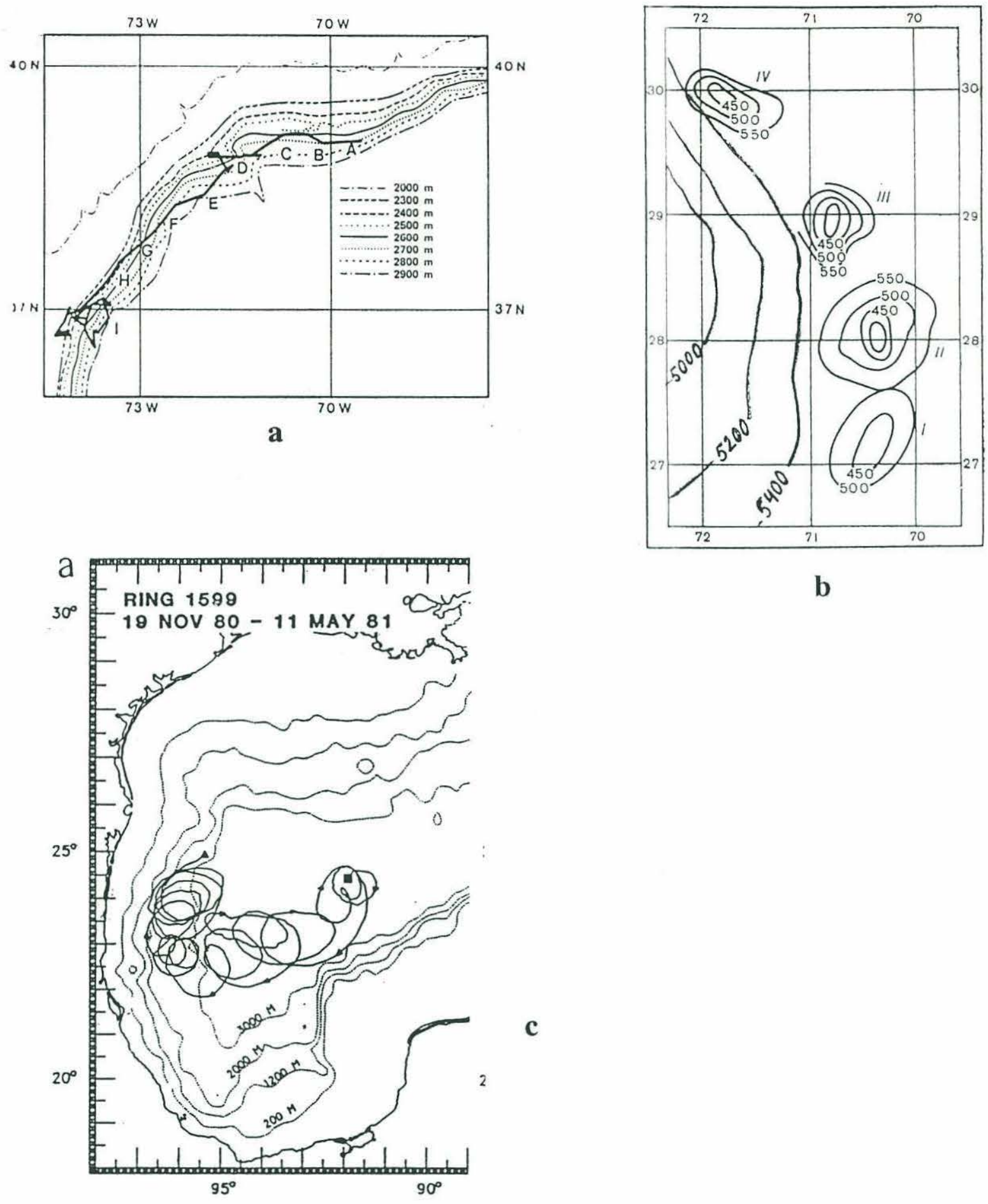

Figure 1.1. Observations of vortices over continental slopes.

(a) - the trajectory of the Gulf Stream WCR-82B (from Evans et al, 1985).

(b) - several successive observations of the subsurface cyclonic vortex in the Sargasso Sea (from Ivanov et al, 1980).

(c) - the trajectory of a surface drifter in the Gulf of Mexico anticyclonic ring (from Kirwan et al, 1985). 


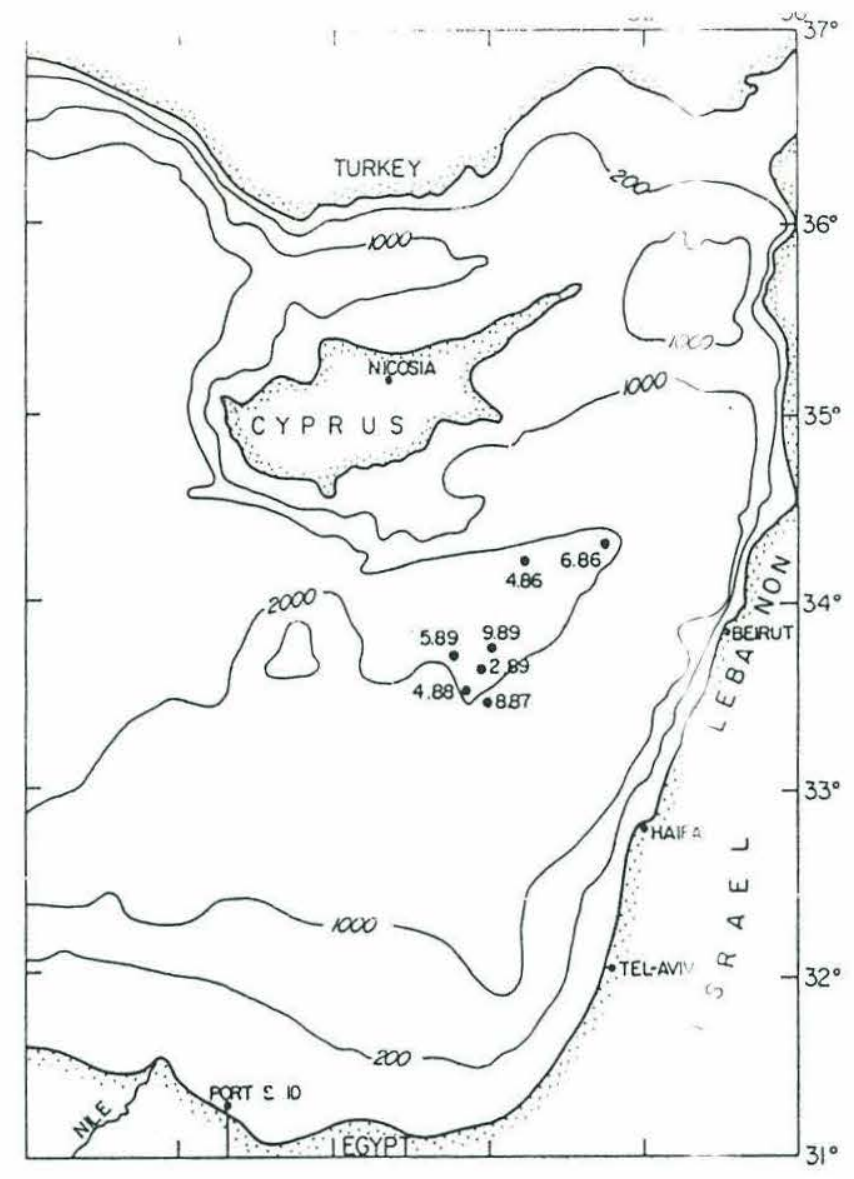

Figure 1.2. Positions of the "Shikmona eddy" in the Levantine basin, observed during several years (from Brenner et al, 1991). 
Interesting eddy-topography interactions can also be encountered in other parts of the ocean. One example is the "Shikmona eddy" - a conspicuous feature of the Levantine basin circulation in the Eastern Mediterranian. For many years this strong compact eddy was observed in the Levantine basin within closed contours of the bowl-shaped topography of this region (see figure 1.2), despite persistent ambient flows that could otherwise carry it away. The vertical size of the eddy core was about $500 \mathrm{~m}$, yet it apparently had difficulties crossing the $1000 \mathrm{~m}$ isobath of this basin. Again, there are no comprehensive observations of deep flows under the eddy but a strong topographic influence is the most likely explanation of its behavior.

The above considered examples show that despite numerous observations of vortices over a varying bottom relief it is difficult to determine the way they interact with topography. This is primarily because of the lack of deep flow measurements under eddies. Often vortices tend to move along isobaths although the direction of their motion varies in different places and circumstances. There are some indications that anticyclones prefer to stay in deeper parts of the ocean while cyclones can be attracted by shallowing bathymetry. This tendency is strongly supported by many theoretical and numerical studies (including the present one) which we shall discuss later. Still the observational evidence of this is not conclusive and requires a more careful analysis.

\subsection{Review of previous work}

The interaction of mesoscale vortices with bottom topography is, as we already mentioned, a less studied area than the influence on vortices of such factors as the planetary beta-effect or background shear flows. It appears to be a more complicated problem because of a wide range of geometric structures possible for a seafloor and three-dimensional baroclinic effects in the case of a stratified ocean.

Historically the first approach to combine mesoscales with bottom topography was mainly statistical, involving numerical experiments with two-dimensional or geostrophic turbulence and a random relief. Barotropic (2-dimensional) turbulence with topography was studied by Bretherton and Haidvogel (1976), Herring (1977) and more recently by Carnevale et al (1991). It was observed from numerical experiments that a flow is significantly modified by non-uniform bottom topography. In particular substantial correlations between the streamfunction and the depth was noticed for the case of strong topographic variations. It is also worth mentioning here that analytical calculations by Holloway (1992) of a "maximum entropy" state for such turbulence results in the 
mean streamfunction being non-zero and simply proportional to a topographic height for scales larger than a deformation radius. Also noted was the obstruction of the inverse energy cascade to larger scales due to the bottom roughness.

The two-layer case, incorporating baroclinic effects, was first studied by Rhines (1977). His results suggest that not only energy cascade towards large scales is restricted in the case of substantial depth variations, but the whole "barotropization mechanism" proposed by him for a flatbottom geostrophic turbulence is also reversed. A flow tends to remain baroclinic and often decoupled in different layers when topography is strong enough. The ratio of barotropic to baroclinic kinetic energy was noted to be a monotonic decreasing function of one important parameter of topographic forcing - Rossby number divided by typical relative depth variations.

Forced geostrophic turbulence was studied by Treguier and Hua (1988). They found the significant dependence of the flow statistics on the initial conditions and on the parameters of forcing. In addition to the baroclinic cascade they observed also the transfer of the barotropic energy towards smaller scales. They also noted that their results depended mainly on a characteristic slope angle rather than total height variations.

Recent numerical experiments by Treguier and McWilliams (1990) and also by Wolff and Maier-Reimer (1991) in a periodic zonal channel involved wind forcing as well as a better resolution of baroclinic effects in the former work (their quasigeostropic model of the Antarctic circumpolar current had three or four layers). Among other factors they investigated the penetration of eddy energy into lower layers and the role of random topography in creating a "bottom form stress" and slowing down the mean current. They also noted a tendency of the eddy field to be baroclinic and the importance of topographic forcing in the zonal momentum balance primarily due to vorticity and not frictional effects. In particular when topography was present the ACC mean flow was an order of magnitude weaker than for the flat-bottom case.

The interaction of individual eddies with topography was studied mainly along several distinct lines.

The first type is "purely bottom" eddies - isolated blobs of cold water lying directly on a sloping bottom under a deep (and usually inactive) layer. Motion of such eddies was considered, for example, by Nof (1983), Swaters and Flierl (1991). These models suggested that such eddies should move "westward", that is along isobaths to the left of the upslope direction. The speed of their motion is proportional to a slope angle and a density difference between an eddy and surrounding water. This is the result of a rather simple balance between gravity and coriolis forces. Such a structure represents a consistent solution for an infinitely deep resting ocean, although for a finite depth for an eddy to be isolated the upper layer must have non-zero compensating circulation as pointed out by Flierl (1987). Moving patches of cold water, for which this model can be relevant, were indeed observed at the bottom of the ocean. But this approach is clearly inappropriate for 
upper-ocean eddies with apparently more complicated dynamics than a simple gravity-coriolis balance.

Another group of studies was concerned with a topographic wave response on a continental slope to an off-shore eddy forcing. This was motivated by numerous observations of energetic topographic wave surges on the continental slope and rise in the Gulf Stream region. Often bursts of topographic waves were associated with Gulf Stream meanders or passages of warm core rings along the slope (Kelley and Weatherly, 1985). Louis and Smith (1982) considered a barotropic problem of topographic wave radiation from a point source of vorticity. They obtained a good agreement with the observed wave pulse during the appearance of the warm core ring in the area of Nova Scotia continental rise. However their model contained several "fitting" parameters which could be used rather arbitrarily to achieve the desired result.

Chapman and Brink (1987) and also Qui (1990) considered a wave response in a stratified case when an eddy can move along a slope. These models prescribe the eddy forcing artificially, without considering the influence of a wave field on a vortex motion itself.

The interaction (in the full meaning of this word) of vortices with a sloping bottom (with a feedback from a topographic radiation field) was studied in several works. A barotropic problem is easier in solving and interpreting results. A uniformly sloping bottom is equivalent dynamically to the planetary beta-effect (with a topographic beta $\beta_{\mathrm{t}}=\alpha \mathrm{f} / \mathrm{H}$ where $\alpha$ - a slope angle and $\mathrm{H}-\mathrm{a}$ reference depth). Although a slope curvature and irregularities can introduce some diversity and complications it is still easier to understand eddy motion in terms of relative vorticity - topographic stretching balances similarly to relative - planetary vorticity balances on a beta-plane.

Carnevale et al (1991) performed laboratory experiments with barotropic cyclones in a conically-shaped basin. Their results clearly demonstrated similarity to the planetary beta-effect: cyclones moved out of the conical valley in anticlockwise spirals and toward the conical hill in clockwise spirals. This means the local "northwest" direction in both cases. Two types of vortices in study differed somewhat in behavior: "sink" vortices with a relatively simple monopolar structure exhibited smooth trajectories while motion of "stirring" vortices with a non-monotonic vorticity distribution showed some loops and wiggles due to a more complicated process of shedding the outer anticyclonic vorticity.

Wang (1992) studied interaction of a barotropic vortex with a simple step-like topography by a contour dynamics method and with a continuous straight-line slope with a planetary beta-effect using a shallow-water numerical model. His results also suggest that cyclonic vortices are prone to upslope motion while anticyclones tend to move away from a slope into deeper waters. Cyclones can interact strongly with shelf waters, wrapping these waters around themselves and inducing a significant cross-shelf transport. Anticyclones can exhibit rather complicated looping and cyclical 
motion when the westward drift caused by the planetary beta-effect counteracts with the downslope tendency.

A baroclinic problem was studied in details by O'Brien and Smith (1983) and by Smith (1986) using a two-layer primitive equations model. As in a barotropic case cyclones tended to move towards shallow waters while anticyclones tried to avoid them. However the baroclinic effects were very important in their numerical experiments. Upper layer vortices usually developed a compensating circulation in a lower layer so that flow cascaded towards a more baroclinic state instead of more barotropic one for a flat-bottom case. This baroclinic compensation was not always complete and they did not study this process in details. But still in all their experiments this oppositesigned circulation played an important role in motion of an initially upper-layer vortices. Cyclones often moved "eastward" - to the right of the upslope direction and slowly drifted upslope while anticyclones often moved near the base of the slope in some irregular loops. O'Brien and Smith (1983) proposed a simple scheme explaining some features of the eddy motion: planetary or topographic beta-effect cause vortices to move "westward" while another, nonlinear tendency shifts their trajectory to the north (upslope) for cyclones and southward (downslope). When the slope is in the meridional direction the planetary and topographic tendencies act perpendicular to each other as shown in the figure 1.3. The arrows in this figure show directions of motion of vortex due to these two factors - the $\beta$-effect and the topographic slope, which is considered as a "topographic $\beta$-effect" and denoted as $\beta_{t}$ in this figure. These effects are added together and this can result in a somewhat complicated behavior. The "nonlinear tendency" (NL) in their scheme is explained by dispersion effect - different "westward" velocities, faster for long and slower for shorter waves. Because of the dispersion disturbances on the "western" side of a vortex quickly radiate away. Shorter waves move very slowly and because a vortex itself moves in the "western" direction these shorter waves trail behind on the "eastern" side of a vortex. Thus disturbances on the "western" side spread over large area and do not influence a vortex significantly. But those on the "eastern" side tend to be more concentrated and their influence on a vortex is stronger. This asymmetry causes the corresponding "meridional" motion of vortices. Below we shall compare our results with this mechanism and propose another, although not completely different scheme to explain the behavior of vortices over a slope.

Recently Shaw and Divakar (1991) considered the interaction of a ring-type vortex over a slope with a better vertical resolution using the semi-spectral primitive equations model with seven vertical modes, which allows to represent baroclinic effects more accurately. They paid attention mainly to the geostrophic adjustment and initial wave radiation process without studying long-term motion of vortices. Yet their results clearly indicate the development of a compensating circulation in deeper waters so that a total barotropic component almost vanishes. 
Summarizing this short review we would like to repeat some important results and tendencies observed in most of these studies. Barotropic models show a clear resemblance to the planetary $\beta$ effect even for cases with a more complicated geometry. Both barotropic and baroclinic models demonstrate an antisymmetric behavior for cyclonic and anticyclonic vortices: while cyclones tend to climb upslope to shallow parts of a basin anticyclones try to avoid shallow waters. But baroclinic effects are evidently important in models with stratification. Upper ocean vortices develop a compensating circulation near the bottom and their motion results from the interaction with this opposite-signed vorticity field. It is this baroclinic mechanism which will be the main subject of our present study.

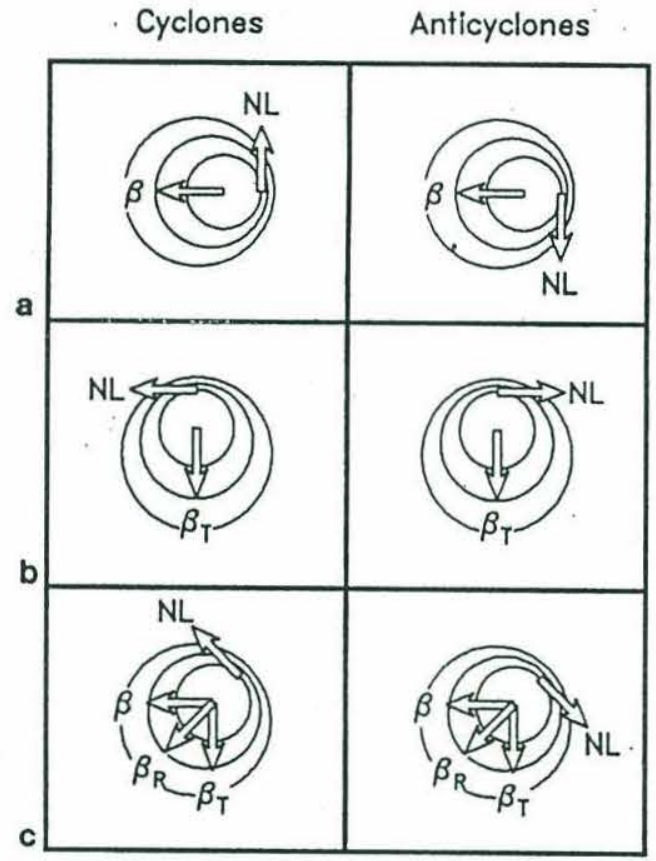

Planetary $\beta$

Topographic $\beta_{\mathrm{T}}$ for upslope to west

Resultont $\beta_{R}$ (combined $\beta$ \& $\beta_{\mathrm{T}}$ )

Figure 1.3. Schematic propagation tendency diagram (From O'Brien and Smith, 1983):

(a) beta-plane, no topography;

(b) topographic $\beta$, f-plane;

(c) combined beta effects (planetary and topographic). 


\section{Chapter 2}

\section{Mesoscale flows over topography:}

\section{some theoretical considerations}

\subsection{Scaling of quasigeostrophic motion with topography}

The purpose of this section is to reexamine the validity of the quasigeostrophic approximation for mesoscale oceanic flows in the presence of bottom topography. We do not attempt here to make another complete ab initio derivation of quasigeostrophy but instead will discuss limitations and constraints introduced by inclusion of a variable relief, using a rather heuristic approach and some available knowledge of the actual ocean dynamics. We shall consider the range of situations when all terms neglected in QG approximation will not exceed the standard limitation of quasigeostrophic dynamics - e. g. Rossby number. This can help determining whether we are within the range of validity of the quasigeostrophic approximation, although it does not validate its use in the rigorous sense.

Consider the equations for rotating stratified frictionless fluid with bottom topography:

$$
\begin{aligned}
\mathbf{u}_{\mathrm{t}}+\left(\mathbf{u} \cdot \nabla_{2}\right) \mathbf{u}+\mathrm{w} \mathbf{u}_{\mathrm{z}}+\mathrm{f} \mathbf{k} \times \mathbf{u} & =-\frac{1}{\rho_{0}} \nabla_{2} \mathrm{p} \\
\mathrm{w}_{\mathrm{t}}+\left(\mathbf{u} \cdot \nabla_{2}\right) \mathrm{w}+\mathrm{ww}_{\mathrm{z}}-\frac{\rho}{\rho_{0}} \mathrm{~g} & =-\frac{1}{\rho_{0}} \mathrm{p}_{\mathrm{z}} \\
\rho_{\mathrm{t}}+\left(\mathbf{u} \cdot \nabla_{2}\right) \rho+w \rho_{\mathrm{z}} & =\frac{\rho_{0}}{\mathrm{~g}} \mathrm{~N}^{2} \mathrm{w} \\
\mathrm{u}_{\mathrm{x}}+\mathrm{v}_{\mathrm{y}}+\mathrm{w}_{\mathrm{z}} & =0
\end{aligned}
$$

and the boundary condition (no normal flow) at the bottom which we allow at this moment to have an arbitrary height $\mathrm{b}(\mathrm{x}, \mathrm{y})$ :

$$
\mathbf{u} \cdot \nabla_{2} \mathrm{~b}-\mathrm{w}=0 \quad @ \mathrm{z}=\mathrm{b}(\mathrm{x}, \mathrm{y})
$$


In the above system u - a horizontal, w - a vertical velocity, $\rho_{0}(\mathrm{z})$ - a reference density in the Boussinesq approximation, $\rho$ - a density perturbation, $\nabla_{2}$ - a horizontal gradient operator.

Now let's choose characteristic scales for a horizontal velocity $\mathbb{U}$, a vertical velocity $\mathbb{W}$, $\mathbb{L}$ - for a horizontal lengthscale, $\mathbb{H}^{*}$ - for a characteristic scale of vertical changes (which does not necessarily coincide with the average depth of an ocean $\mathbb{H}_{0}$ ), $\mathbb{T}$ - a characteristic timescale (independent and not necessarily equal to the advective timescale $\mathbb{U} / \mathbb{L}$ ) and $\alpha$ - a typical angle of a bottom slope $\left(\nabla_{2} \mathrm{~b}\right)$; also $\mathbb{P}$ and $\rho$ are the scales for pressure and density perturbations respectively. $\mathbb{N}$ will be a scale for a buoyancy frequency. Using these characteristic scales we can write a scaling table for each term in the system (2.1):

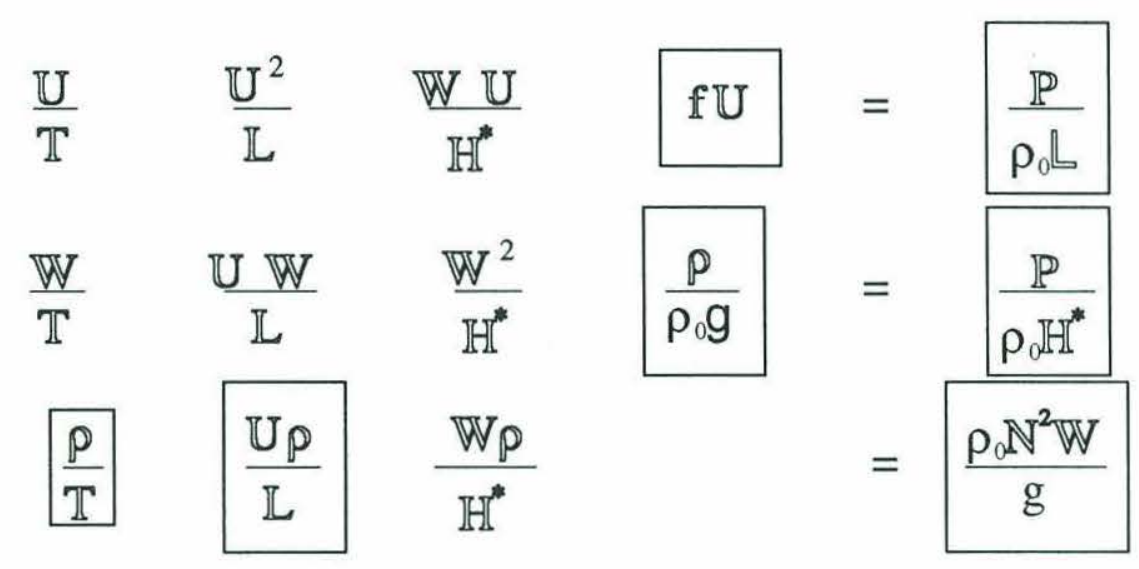

For continuity we have

$$
\frac{\mathbb{U}}{\mathbb{L}} \quad \frac{\mathbb{U}}{\mathbb{L}}=\frac{\mathbb{W}}{\mathbb{H}^{*}}
$$

and for the boundary condition

$$
\mathbb{U} \sin \theta=\mathbb{W}
$$

where $\theta$ - a characteristic angle between horizontal velocity and isobaths. It would be simpler to estimate $\sin \theta$ as 1 and not include the angle $\theta$ at all among the scaling parameters. Still we would like to keep in mind that this angle can be not completely arbitrary. Near steep topography a velocity usually tend to align along isobaths and so $\theta$ can be small, thus allowing a strong horizontal flow without causing too large vertical velocities. 
We assume an approximate balance of the terms corresponding to the QG dynamics (scaling for them are put in frames in (2.2)) and consider the importance of other terms in (2.1) relative to the terms in this basic $(\mathrm{QG})$ balance. To do so we divide each row in $(2.2 \mathrm{a}-\mathrm{c})$ by a one of the basic terms:

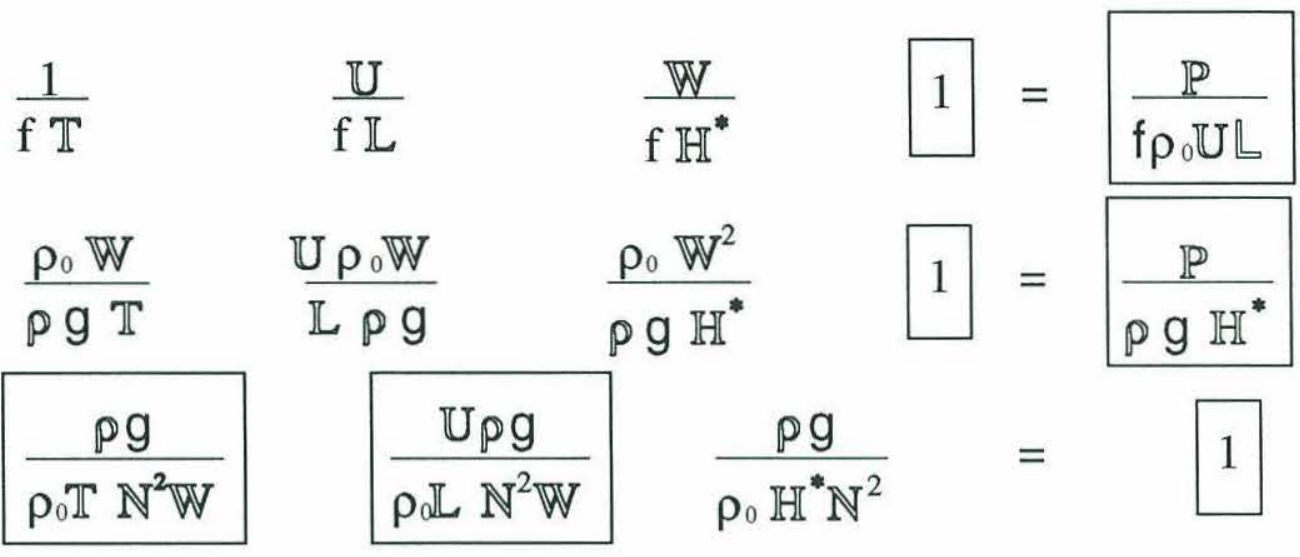

From the approximate QG balance we immediately obtain a scaling for $\mathbb{P}$ and $p$ :

$$
\mathbb{P}=f \rho_{0} \mathbb{U} L, \quad \frac{\mathbb{P}}{\rho_{0}}=\frac{f \mathbb{U} L}{g \mathbb{H}^{*}}
$$

and the table $(2.2 \mathrm{a}-\mathrm{c})$ becomes:

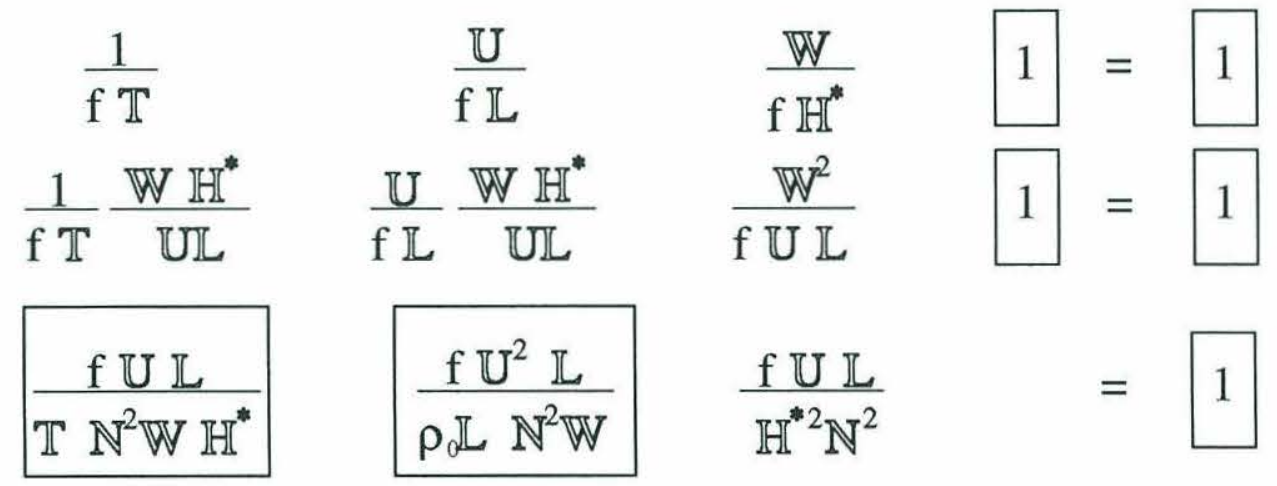

Now we shall define dimensionless parameters traditionally used in the geophysical fluid dynamics:

$$
\begin{aligned}
& \frac{1}{\mathrm{f} \mathbb{T}} \equiv \mathbb{R}_{\mathbb{T}}-\text { temporal Rossby number, } \\
& \frac{\mathbb{U}}{\mathrm{f} \mathbb{L}} \equiv \mathbb{R}_{\mathbf{A}} \text { - advective Rossby number, }
\end{aligned}
$$




$$
\frac{\mathbb{N}^{2} \mathbb{H}^{* 2}}{\mathrm{f}^{2} \mathbb{L}^{2}} \equiv \mathbb{B} \text { - Burger number }
$$

With these definitions our table takes the following form:

$$
\begin{array}{ccccc}
\mathbb{R}_{\mathrm{T}} & \mathbb{R}_{\mathbf{A}} & \mathbb{R}_{\mathbf{A}} \frac{\mathbb{W} \mathbb{L}}{\mathbb{U} \mathbb{H}^{*}} & 1 & =1 \\
\mathbb{R}_{\mathrm{T}} \frac{\mathbb{W} \mathbb{H}^{*}}{\mathbb{U} \mathbb{L}} & \mathbb{R}_{\mathbf{A}} \frac{\mathbb{W} \mathbb{H}^{*}}{\mathbb{U} \mathbb{L}} & \mathbb{R}_{\mathbf{A}} \frac{\mathbb{W}^{2}}{\mathbb{U}^{2}} & 1 & =1 \\
\frac{\mathbb{R}_{\mathrm{T}} \frac{\mathbb{U} \mathbb{L}}{\mathbb{W} \mathbb{H}^{*}} \frac{f^{2}}{\mathbb{N}^{2}}}{\mathbb{R}_{\mathbf{A}} \frac{\mathbb{U} \mathbb{L}}{\mathbb{W} \mathbb{H}^{*}} \frac{f^{2}}{\mathbb{N}^{2}}} \frac{\mathbb{R}_{\mathbf{A}}}{\mathbb{B}} & =1
\end{array}
$$

Now we consider scaling of the continuity equation and the boundary condition and scaling of the vertical velocity associated with it. Comparison of either of the first two terms in (2.2d) with the third term gives an often used characteristic ratio of the vertical to the horizontal velocity as an inverse ratio of the corresponding lengthscales: $\mathbb{W} / \mathbb{U}=\mathbb{H}^{*} / \mathbb{L}$. For the QG dynamics it is not a proper estimate because the horizontal velocity is almost nondivergent, $\mathbf{u}_{x}+\mathbf{v}_{\mathbf{y}} \cong 0$ and the first two terms in (2.2d) compensate each other to the order of a Rossby number. To be more specific we shall denote it $\mathbb{R}_{\mathfrak{c}}$, "c" - for "continuity" and define it as

$$
\mathbb{R}_{c} \equiv \frac{\mathbb{W} / \mathbb{U}}{\mathbb{H}^{*} / \mathbb{L}} \text {, so that for a vertical velocity we get } \mathbb{W}=\mathbb{R}_{c} \mathbb{H}^{*} \frac{\mathbb{U}}{\mathbb{L}} \text {, }
$$

and for the frequent combinations $\quad \frac{\mathbb{U} \mathbb{L}}{\mathbb{W} \mathbb{H}^{*}}=\frac{1}{\mathbb{R}_{\mathbf{c}}} \frac{\mathbb{L}^{2}}{\mathbb{H}^{* 2}}, \quad \frac{\mathbb{U} \mathbb{L}}{\mathbb{W} \mathbb{H}^{*}} \frac{f^{2}}{\mathbb{N}^{2}}=\frac{1}{\mathbb{R}_{\mathbf{c}} \mathbb{B}}$

Now our scaling table can be rewritten as following: 


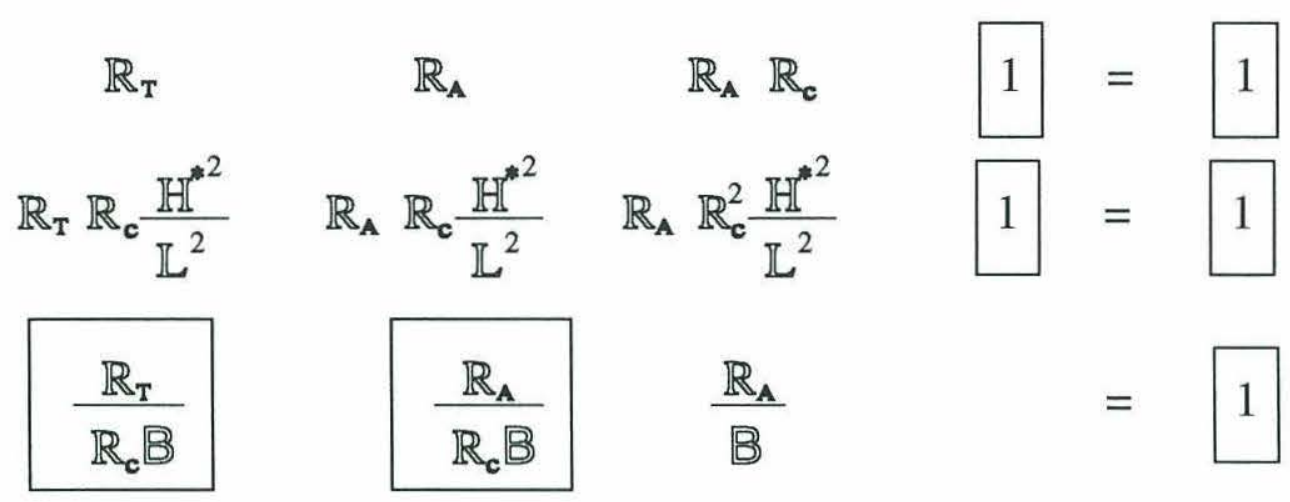

- From the first row (2.5a) (horizontal momentum balance) we obtain the usual requirement of the smallness of Rossby numbers

$$
\mathbb{R}_{\mathrm{T}}, \mathbb{R}_{\mathrm{A}} \ll 1
$$

- Provided this and relative smallness of the vertical scale $\mathbb{H}^{*} \leq \mathbb{L}$ the first three terms in the second row (2.5b) (a vertical momentum balance) are very small which implies a high degree of validity of the hydrostatic approximation.

- From (2.5c) corresponding to the mass conservation equation we immediately get another QG constraint $\mathbb{R}_{\mathbf{A}} / \mathbf{B}<1$ (small relative isopycnal displacement) and a relation for the continuity Rossby number

$$
\mathbb{R}_{\mathrm{c}}=\frac{\max \left(\mathbb{R}_{\mathrm{T}}, \mathbb{R}_{\mathbf{A}}\right)}{\mathbb{B}}
$$

Analyzing this last relation more carefully it can be argued that the temporal Rossby number is more relevant in it than the advective one. When motion is rather strong and nonlinear, near the limits of validity of QG dynamics, the term corresponding to advection of density perturbations is actually smaller than its simple scaling estimate given by the second term in the $(2.5 \mathrm{c})$. This may occur in some energetic geostrophic motions when a horizontal pressure gradient is approximately parallel to a horizontal density gradient (both are directed perpendicular to a strong jet with a density front or radially in a mesoscale eddy). However this is only an assumption and one should not expect it to hold for all cases. It depend also on certain symmetries of the flow, such as the radial symmetry for eddies or smallness of meander velocities relative to along-front velocity for oceanic jets. But it may be true for a broad class of oceanic motions. In particular, the "equivalent barotropic mode", for which velocities are parallel to the density gradient, was pointed out by Killworth (1992) 
in analyzing ACC velocity fields. In such cases the second term in (2.1c) is smaller than its simple scaling (2.5c).

Another way of saying this is that the biggest ratio of the vertical to horizontal quasigeostrophic velocity is associated typically with time-dependence and not with strong but steady and symmetric horizontal motions. In the light of this consideration the continuity Rossby number will be given simply by

$$
\mathbb{R}_{\mathrm{c}}=\frac{\mathbb{R}_{\mathrm{T}}}{\mathbb{B}}
$$

Now we want to concentrate on the role of topography in these scaling exercises. When bottom topography is present we should add new scaling constraints:

- The horizontal scale of topographic features $\mathbb{L}_{b}$ should not exceed the horizontal lengthscale of the flow $\mathbb{L}$; we can introduce yet another (topographic) Rossby number $\mathbb{R}_{b}$, (b for "bottom") which must be small $\quad \mathbb{R}_{\mathfrak{b}}=\mathbb{U} / \mathrm{f} \mathbb{L}_{\mathbf{b}}<<1$.

- Characteristic timescale considered independent until now is determined by the topographic Rossby waves frequency: $\quad \omega=\frac{1}{\mathbb{T}} \leq \frac{\mathbb{N} \propto}{\tanh \left(\frac{\mathbb{H}\left(\mathbb{N}_{0} \mathbb{N}\right.}{\mathbb{L f}}\right)}, \quad$ so that

$$
\mathbb{R}_{\mathrm{T}}=\frac{1}{\mathrm{f} \mathbb{T}} \leq \frac{\mathbb{N}_{\boldsymbol{Q}}}{\mathrm{f}} \frac{1}{\tanh \left(\frac{\mathbb{H}_{0} \mathbb{N}}{\mathbb{L}_{\mathrm{f}}}\right)}
$$

This imposes a constraint on the slope angle which, rescaled by $\mathbb{N} / \mathbb{f}$ factor, should be small:

$$
\frac{\mathbb{N}_{\mathfrak{f}}}{\mathrm{f}}<<\tanh \left(\frac{\mathbb{H}_{0} \mathbb{N}}{\mathbb{L} f}\right) \leq 1
$$

- The vertical velocity set by the bottom boundary condition must be consistent with QG dynamics, that is it should not exceed the quasigeostrophic vertical velocity derived from the continuity equation (or the mass conservation equation). This is equivalent to the relation

$$
\mathbb{R}_{\mathrm{c}} \frac{\mathbb{H}^{*}}{\mathbb{L}} \geq \boldsymbol{\alpha} \sin \theta \quad \text { or } \quad \frac{\mathbb{R}_{\mathrm{T}}}{\mathbb{B}} \frac{\mathbb{N} \mathbb{H}^{*}}{\mathrm{f} \mathbb{L}} \geq \frac{\mathbb{N}_{\mathrm{f}}}{\alpha \sin \theta}
$$


where $\theta$ is the maximal angle between the streamlines at the bottom and isobaths.

Assuming also that for the topography - controlled regime $\mathbb{H}^{*}=\mathbb{H}_{0}$ we obtain the following restriction on the rescaled slope angle:

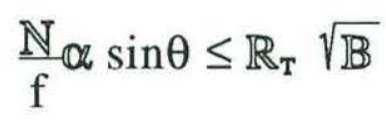

Using (2.7) and estimating $\sin \theta$ as 1 we get

$$
\frac{\mathbb{N H}^{*}}{\mathrm{f} L} \tanh \left(\frac{\mathbb{N H}_{0}}{\mathrm{f} L}\right)<1
$$

This is equivalent to $\sqrt{\mathbb{B}} \tanh (\sqrt{\mathbb{B}}) \approx 1$ or, simply

$$
\mathbb{B} \approx 1
$$

This estimate of the Burger number (the "Prandtl balance") is very natural for the interaction of an upper-ocean mesoscale flow with topography, because the characteristic horizontal scale corresponds to a deformation radius which is in turn scaled as an ocean depth multiplied by the $\mathbb{N} / \mathbb{f}$ factor.

Topographic limitations as we have seen above are imposed primarily through the restrictions on the characteristic slope angle $\boldsymbol{\alpha}$. These restrictions are associated with two different factors topographic waves frequency and scaling for a vertical velocity in the continuity and mass conservation equations. However there can be quite realistic situations when these restrictions may be relaxed.

First - if a flow is steady or evolving slowly enough, without significant radiation of topographic waves, their period will not be a characteristic timescale of a process and will not limit validity of the QG approximation.

Second - in the case of rather steep topography a flow at the bottom is likely to be almost parallel to the isobaths (again when it is nearly steady and not very strong at depths) so that $\sin \theta$ in (2.8) is small and $\alpha \mathbb{N} / \mathrm{f}$ does not necessarily have to be small. This factor, although speculative, can help QG dynamics extend its validity to a larger variety of topographies and rather realistic oceanic conditions. We do not attempt here to devise a general a priori condition for applicability of these arguments. The proposed recipe instead is to look at a given solution for a specific situation and use these ideas to check the consistency of the flow with the quasigeostrophic approximation. 
These considerations indicate that flows with the characteristic Burger number of the order 1 (which is rather natural than a restrictive condition) and smooth topography with typical slopes

$\propto \frac{\mathbb{N}}{\mathrm{f}} \approx \max \left(\mathbb{R}_{\mathbf{A}}, \mathbb{R}_{\mathrm{T}}\right)$ and horizontal scales of order $(\mathbb{L})$ fall within the reach of QG dynamics. By this we mean that all terms in the system (2.1) which are not included in the QG balance are no larger than temporal or advective Rossby numbers, and the error due to all ageostrophic terms does not exceed the maximum of this two numbers. One can argue however that we can not consistently consider the quasigeostrophic approximation in a domain larger than $\mathbb{L} / \mathbb{R}$, and then smallness of a slope implies smallness of topographic height variations themselves. This can be true in many situations although when the flow only in the middle of a domain is important (like in our case of a single vortex over a slope) one can probably use a domain of a bigger size if necessary. In the next section we present a two-scale derivation of quasigeostrophic approximation for a case when bottom topography varies slowly, but still overall height variations can be large enough. And generally we argue here that there are quite realistic situations when even these restrictions can be relaxed and a flow over rather steep and prominent topographic features can stay well within the validity range of quasigeostrophy, understood in a somewhat broader than usual sense.

\subsection{The bottom boundary condition}

In the previous section we argued that the quasigeostrophy can hold rather well in certain situations even in the case of order one topographic variations. If this is so it naturally poses a question about the bottom boundary condition in a QG model. Traditionally the quasigeostrophic approximation was derived under the assumption of small topographic variations and the boundary condition was related to a reference depth instead of the actual position of a seafloor in the ocean. But if we want to allow (with necessary caution discussed in the preceding section) larger variations of topography this reference depth approximation looses consistency. Apparently it is the local depth that matters at a given point, and a reference depth can differ substantially from it. One can simply say that this reference depth should be abandoned and use actual depth instead. Still because formal rigorous derivations of quasigeostrophy and its properties (like PV and energy conservation) were always performed with a reference depth assumption one wish to substantiate switching to an actual depth in the bottom boundary condition with some theoretical basis.

In this section we attempt to justify the use of an actual depth using a simplified version of the two-scale approach of, for example, Pedlosky (1984). The idea of the following derivations was 
actually suggested by G. Flierl (personal communication). Our central assumption will be that the bottom elevation varies smoothly, at the characteristic lengthscale considerably larger than other relevant horizontal scales, e. g. a deformation radius. It allows us to introduce a pair of slowly varying horizontal coordinates $\mathrm{X}$ and $\mathrm{Y}$ so that the bottom elevation will depend exclusively on them, instead of the "local" coordinates $\mathrm{x}$ and $\mathrm{y}$. We shall nondimensionalize "local" and "global" coordinates in the following form:

$$
\left(\mathrm{x}^{\prime}, \mathrm{y}^{\prime}\right)=(\mathrm{x}, \mathrm{y}) / l, \quad(\mathrm{X}, \mathrm{Y})=(\mathrm{x}, \mathrm{y}) / \mathrm{L}
$$

Here $\left(x^{\prime}, y^{\prime}\right)$ and $(X, Y)$ are nondimensional and their ratio will be a small parameter:

$$
\delta=l / \mathrm{L}
$$

Time $\mathrm{t}$ will be nondimensionalized by the advective scale:

$$
\mathrm{t}^{\prime}=\mathrm{t} l / \mathrm{U}
$$

Now we let our variables $\quad \mathrm{u}, \mathrm{v}, \mathrm{w}, \mathrm{p}, \rho$ depend formally on both horizontal scales: "local" - $\mathrm{x}, \mathrm{y}$ and "global", slowly varying $\mathrm{X}, \mathrm{Y}$. Dimensionless variables will be written as follows:

$$
\begin{aligned}
u & =U u^{\prime}\left(x^{\prime}, y^{\prime}, z^{\prime}, t^{\prime}, X^{\prime}, Y^{\prime}\right) \\
v & =U v^{\prime}\left(x^{\prime}, y^{\prime}, z^{\prime}, t^{\prime}, X^{\prime}, Y^{\prime}\right) \\
w & =U \omega w^{\prime}\left(x^{\prime}, y^{\prime}, z^{\prime}, t^{\prime}, X^{\prime}, Y^{\prime}\right)
\end{aligned}
$$

Here $\omega$ is the ratio of vertical to horizontal velocity scales. We also let our coriolis parameter $\mathrm{f}$ depend on the "global" variable $\mathrm{Y}$ so that we shall nondimensionalize it in the form:

$$
\mathrm{f}=\mathrm{f}_{0} \mathrm{f}^{\prime}(\mathrm{Y})
$$

where $\mathrm{f}_{0}$ is simply twice the Earth rotation rate and $\mathrm{f}^{\prime}$ is the sine of latitude (the same as in Pedlosky, 1984). For pressure and density perturbations we shall have:

$$
\begin{aligned}
& \mathrm{p}=\rho_{0} \mathrm{f}_{0} \mathrm{U} l \mathrm{p}^{\prime} \\
& \rho=\rho_{0} \frac{\mathrm{f}_{0} \mathrm{U} l}{\mathrm{~g}} \omega \rho^{\prime}
\end{aligned}
$$


As usual we shall introduce the Rossby number $\quad \mathrm{R}=\frac{\mathrm{U}}{\mathrm{f}_{0} l} \quad$ (here for simplicity we shall not distinguish between different Rossby numbers considered in the previous section).

Horizontal derivatives will be transformed according to:

$$
\frac{\partial}{\partial \mathrm{x}}=\frac{1}{l}\left(\frac{\partial}{\partial \mathrm{x}^{\prime}}+\delta \frac{\partial}{\partial \mathrm{X}}\right), \quad \frac{\partial}{\partial \mathrm{y}}=\frac{1}{l}\left(\frac{\partial}{\partial \mathrm{y}^{\prime}}+\delta \frac{\partial}{\partial \mathrm{Y}}\right)
$$

Equations of motion will be written in the following form (dropping primes for simplicity):

$$
\begin{gathered}
\mathrm{R}\left(\mathbf{u}_{\mathrm{t}}+\mathbf{u} \nabla \mathbf{u}+\delta \mathbf{u} \nabla \mathbf{u}+\omega \mathbf{u}_{\mathrm{z}}\right)+\mathrm{fk} \times \mathbf{u}=-\nabla \mathrm{p}-\delta \nabla \mathrm{p} \\
\nabla \cdot \mathbf{u}+\delta \nabla \cdot \mathbf{u}+\omega \mathrm{w}_{\mathrm{z}}=0 \\
\mathrm{R}\left(\rho_{\mathrm{t}}+\mathbf{u} \nabla \rho+\delta \mathbf{u} \nabla \rho\right)+\omega \mathrm{N}^{2} \mathrm{w}=0
\end{gathered}
$$

And the boundary conditions:

$$
\begin{array}{clll}
\omega \mathrm{w}=\delta \mathbf{u} \nabla \mathrm{b} & @ \mathrm{z}=\mathrm{b}(\mathrm{X}, \mathrm{Y}) & \text { (bottom) } \\
\mathrm{w}=0 & @ \quad \mathrm{z}=\mathrm{H} \quad \text { (surface) }
\end{array}
$$

In the above equations we used the notation $\nabla$ for the gradient operator with respect to coordinates $(\mathrm{X}, \mathrm{Y}), \quad \nabla=\left(\frac{\partial}{\partial \mathrm{X}}, \frac{\partial}{\partial \mathrm{Y}}\right)$, and bold $\mathbf{u}$ stands for the vector of horizontal velocities $\mathbf{u}=(\mathrm{u}, \mathrm{v})$.

Our next assumption will be that all the parameters $\delta, R, \omega$ are small and of the same order (for $\omega$ this follows from the bottom boundary condition (2.14d) assuming $\nabla \mathrm{b} 1$ in "global" coordinates):

$$
\delta \sim \mathrm{R} \sim \omega<1
$$

Now we can expand our variables in powers of any of these parameters (we shall stick with the traditional choice of the Rossby number). So, for example, for horizontal velocities we have:

$$
\mathbf{u}=\mathbf{u}_{0}+R \mathbf{u}_{1}+R^{2} \mathbf{u}_{2}+\ldots
$$


Other variables will be expanded in a similar manner. Substituting this into the equations of motion (2.14) and equating terms with the same power of parameters we obtain in the leading order:

$$
\begin{gathered}
\mathrm{f} \mathbf{k} \times \mathbf{u}_{0}=-\nabla \mathrm{p}_{0} \\
\nabla \cdot \mathbf{u}=0 \\
\rho_{0 \mathrm{t}}+\frac{\delta}{\mathrm{R}} \nabla \cdot \mathbf{u}+\frac{\omega}{\mathrm{R}} \mathrm{w}_{0 \mathrm{z}}=0
\end{gathered}
$$

For the first order we have

$$
\begin{gathered}
\frac{\mathrm{D}_{0}}{\mathrm{Dt}} \mathbf{u}_{0}+\mathrm{f} \mathbf{k} \times \mathbf{u}_{1}=-\nabla \mathrm{p}_{1}-\frac{\delta}{\mathrm{R}} \nabla \mathrm{p}_{0} \\
\nabla \cdot \mathbf{u}_{1}+\frac{\delta}{\mathrm{R}} \nabla \cdot \mathbf{u}_{0}+\frac{\omega}{\mathrm{R}} \mathrm{w}_{0 \mathrm{z}}=0
\end{gathered}
$$

To obtain a consistent equation of conservation of the quasigeostrophic potential vorticity we need to use the first-order approximation. Taking the curl of the equation (2.16a) we get:

$$
\frac{\mathrm{D}_{0}}{\mathrm{Dt}} \nabla^{2} \mathrm{p}_{0}+\mathrm{f} \nabla \cdot \mathbf{u}_{1}=-\frac{\delta}{\mathrm{R}}\left(\partial_{\mathrm{Yx}}^{2} \mathrm{p}_{0}-\partial_{\mathrm{Xy}}^{2} \mathrm{p}_{0}\right)
$$

The only term in this last equation containing first-order variable $-\nabla \cdot \mathbf{u}_{1}$ - can be expressed via the first-order continuity equation:

$$
\nabla \cdot \mathbf{u}_{1}=\frac{\delta}{R}\left[\left(\frac{p_{0 y}}{f}\right)_{X}-\left(\frac{p_{0 x}}{f}\right)_{Y}\right]-\frac{\omega}{R} w_{0 z}
$$

Combining these last two equations we obtain the expected QG vorticity balance:

$$
\frac{\mathrm{D}_{0}}{\mathrm{Dt}} \nabla^{2} \mathrm{p}_{0}+\frac{\delta}{\mathrm{R}} \frac{\beta}{\mathrm{f}} \mathrm{p}_{0 \mathrm{x}}=\frac{\omega}{\mathrm{R}} \mathrm{w}_{0 \mathrm{z}}
$$


This equation should be combined with the density conservation equation (with inclusion of a hydrostatic approximation) to provide another relation between zero-order variables $\mathrm{p}_{0}$ and $\mathrm{w}_{0}$ :

$$
\frac{\mathrm{D}_{0}}{\mathrm{Dt}} \mathrm{p}_{0 \mathrm{z}}+\frac{\omega}{\mathrm{R}} \mathrm{N}^{2} \mathrm{w}_{0}=0
$$

And the bottom boundary condition in the lowest order will include a horizontal gradient of the bottom elevation:

$$
\mathrm{w}_{0}=\frac{\delta}{\omega} \mathbf{u}_{0} \nabla \mathrm{b} \quad \text { @ } \mathrm{z}=\mathrm{b}(\mathrm{X}, \mathrm{Y}) \text { (bottom) }
$$

The quasigeostrophic equations (2.17) naturally do not include derivatives with respect to the global coordinates $\mathrm{X}$ and $\mathrm{Y}$. However they depend on $\mathrm{X}$ and $\mathrm{Y}$ parametrically in two places: first - the coriolis parameter $\mathrm{f}$ is a function of $\mathrm{Y}$ and second - the bottom elevation is also a function of global variables. The bottom boundary condition (2.17) is related to the actual depth $\mathrm{z}=\mathrm{b}(\mathrm{X}, \mathrm{Y})$ and not to a reference bottom depth as usually assumed in quasigeostrophy. This is again similar to Pedlosky (1984), who derived the "local" quasigeostrophy which is parametrically dependent on a slowly varying coriolis parameter and a reference stratification profile.

One can expect that in most cases this does not make much difference and we shall see later that in our numerical model the results with the reference and the actual depth are quite similar. However, we shall use this difference explicitly in chapter 3 where we shall consider the possibility of steady motion of a vortex above a uniformly sloping bottom. In this case it appears that such motion can be caused by a broken symmetry in the bottom boundary condition when it is related to the actual depth instead of the reference level.

\subsection{Continuously stratified vs. layered model}

As it was already mentioned above we use a continuously stratified model in this study while to represent effects of stratification and baroclinicity a multi-layered model is more frequently used in oceanographic literature. Since a layered model is certainly a convenient and popular tool and many 
oceanographers feel more comfortable with it we would like to comment on our choice, with a brief comparison of layered and continuous stratification.

One of the advantages of a layered model (as noted by Pedlosky, 1987) is that it is an exact representation of a certain physical system, which is in turn a crude representation of a continuous density field of the real ocean. It can also be easily reproduced in a laboratory experiment.

As a vertical discretization of a continuous density profile it is simple and convenient, although a crude one because it involves exact differences instead of finite-difference approximation of vertical derivatives as, for example, in a "level" model. Detailed comparison of layered and level model can be found in Pedlosky (1987) and need not be repeated here. Another advantage of a layered model is that it uses an isopycnal vertical coordinate which coincides with material surfaces and is often believed to be a more natural choice for ocean modeling.

Still the ocean does not consist of a pile of layers with a uniform density but instead a continuously stratified medium. Therefore the question as to how well can it be represented by a model with a few layers should be addressed. In this context some deficiencies and difficulties of a layered model are worth mentioning, especially those arising in the case of non-uniform bottom topography.

When the quasigeostrophic approximation is used it is necessary to impose rather severe restrictions on topography possible in the model: the height of topographic variations should be small compared to the thickness of the lowermost layer. This diminishes our ability to resolve a baroclinic structure of a flow near the bottom. Since, as we argued in the previous section, limitations of quasigeostrophy are applied to a slope angle rather than a total height variation this is an inconvenient and unnecessary restriction. When in a layered model topography protrudes into the next upper layer so that isopycnal surfaces intersect the bottom an awkward situation arises: the potential vorticity in a "wedge" between a bottom and layer interface goes to infinity. Although it is not prohibited physically and can be dealt with in principle, it presents unnecessary inconvenience, especially in QG case. Attempts to describe this bottom outcropping in comprehensive numerical models involve extrapolations of the pressure gradient from neighboring grid points (for example in Bleck and Smith, 1990) - a procedure of a dubious accuracy and efficiency, which often proved to have the unsatisfactory performance.

In a continuously stratified model we can allow intersections of isopycnal surfaces with topography in a more natural way. Moreover, while oceanographers usually tried to avoid these intersections we are going to make them a primary tool, explicitly using their positions to determine the velocity field, as we shall describe in chapter 4 .

Generally speaking, the comparison of continuously stratified and layered models is not sensible unless we specify the method of vertical discretization for continuous stratification. This is done usually in a "level" form or by expansion into vertical normal modes. The latter method can be 
used only for small topographic variations but for any stratification profiles. For a given vertical resolution it is a more accurate vertical representation than layers, as argued by Flierl (1978), and can be used for "calibration" of a layered model.

But for a simplified problem of interaction of an upper-ocean eddy with a bottom slope in an otherwise quiescent ocean we shall not need any vertical discretization at all. In quasigeostrophic dynamics the coupling between surface flow and the bottom can be traced throughout the whole water column without the information about intermediate water, so the problem practically becomes two-dimensional. This can be used only with a certain class of buoyancy profiles, devoid of real oceanic fine-structure, as we shall consider in the Appendix 1. But the simplification and convenience promised by this approach makes it in our opinion worth following. 


\section{Chapter 3}

\section{Steady motion of a baroclinic point}

\section{vortex along a sloping bottom}

\subsection{Introduction}

In this chapter we explore a possibility of steady motion of a vortex along a uniformly sloping bottom. As we already discussed in chapter 1 , there are many observations of such motion of mesoscale eddies over continental slopes. In these areas the influence of topography could be much larger than that of other factors, like the planetary beta-effect. It is interesting to note that the direction of motion of these eddies is different in various cases. Gulf Stream warm core rings move usually to the left of the upslope direction ("westward"). Other eddies, like Gulf of Mexico rings and also some cyclonic eddies to the south of the Gulf Stream move "eastward" ( to the right of the uphill direction), often following contours of a constant depth quite closely.

It is well known that a monopolar vortex can not move steadily on the planetary $\beta$-plane. Its total angular momentum and hence a potential vorticity must be zero for steady motion. This is the direct consequence of the "zero angular momentum" theorem formulated in Flierl, Stern and Whitehead (1983). Although this theorem is not directly applicable to a case of non-uniform topography, there is a general dynamical equivalence of a planetary $\beta$-plane and constant slope topography for quasigeostrophic dynamics in a homogeneous ocean. This implies that a barotropic vortex can not move steadily along a uniformly sloping bottom. But it is not clear that this is also the case for a stratified ocean, and we shall try to answer this question below.

In this chapter, as throughout most of this thesis, we shall use an f-plane quasigeostrophic approximation with a constant buoyancy frequency. We shall develop a semi-analytical model describing steady motion of a 3-dimensional point vortex (a singular potential vorticity anomaly) placed at the surface or in the bulk of an ocean. We shall find the conditions which make such steady motion possible and obtain the direction and the speed of a vortex depending on its strength, the slope angle and the distance to the surface and the bottom. We shall see that in the QG approximation 
a vortex can move steadily only when the bottom boundary condition is applied at the actual depth rather than at the reference level. Thus the results of this chapter depend strongly on this assumption which makes them somehow questionable. Nevertheless I feel it is worth presenting them here if only to demonstrate this difference in applying the bottom boundary condition.

\subsection{The bottom boundary condition for steady motion}

Consider at first an arbitrary stratified flow between the horizontal upper surface and a uniformly sloping bottom inclined to a horizontal plane at a small angle $\alpha<<1$. Let the origin of a coordinate system be placed somewhere at the bottom with the $\mathrm{x}$ axis directed along the isobath, $\mathrm{y}$ - shoreward into the bottom and the vertical coordinate $\mathrm{z}$ downward (figure 3.1). Corresponding velocities are $\mathrm{u}, \mathrm{v}, \mathrm{w}$. The bottom boundary condition requires a normal to the bottom velocity to be zero:

$$
\alpha v+w=0
$$

The vertical velocity is related to the isopycnal height displacement $h(x, y, t)$ (positive downward):

$$
\mathrm{w}=\frac{\mathrm{dh}}{\mathrm{dt}}=\mathrm{h}_{\mathrm{t}}+\mathrm{uh}_{\mathrm{x}}+\mathrm{vh}_{\mathrm{y}}
$$

If horizontal velocities are geostrophic $-u=-p_{y} / \rho_{0} f, v=p_{x} / \rho_{0} f$, then (3.2) becomes:

$$
w=h_{t}+\frac{1}{\rho_{0} f}\left(p_{x} h_{y}-p_{y} h_{x}\right)=h_{t}+\frac{1}{\rho_{0} f} J(p, h)
$$

If we assume a quasigeostrophic approximation then an isopycnal height displacement is related to a vertical derivative of pressure:

$$
\mathrm{h}=-\frac{1}{\rho_{0} \mathrm{~N}^{2}} \mathrm{p}_{\mathrm{z}}
$$

and we can rewrite (3.3) in the form: 


$$
w=-\frac{1}{\rho_{0} N^{2}}\left[p_{z t}+\frac{1}{\rho_{0} f} J\left(p, p_{z}\right)\right]
$$

Substituting the vertical velocity from (3.3') and the onshore velocity $\mathrm{v}$ into the boundary condition (3.1) we obtain:

$$
\frac{\alpha}{\mathrm{f}} \mathrm{p}_{\mathrm{x}}-\frac{1}{\mathrm{~N}^{2}}\left[\mathrm{p}_{\mathrm{zt}}+\frac{1}{\rho_{0} \mathrm{f}} \mathrm{J}\left(\mathrm{p}, \mathrm{p}_{\mathrm{z}}\right)\right]=0
$$

Now consider special situations when the nonlinear Jacobian term in (3.5) vanishes, so that the pressure (streamfunction) and its vertical derivative are functionally related at the bottom. Firstly, it happens when the pressure is a separable function of horizontal and vertical coordinates:

$$
\mathrm{p}=\psi(\mathrm{x}, \mathrm{y}) \phi(\mathrm{z})
$$

One particular example of such motion is topographic Rossby waves (see Rhines, 1970). From (3.5) it follows that a free monochromatic quasigeostrophic topographic wave even of finite amplitude will remain linear, so it will not generate its higher-order harmonics (this is very similar to the case of planetary Rossby waves). But we consider another, more interesting case, when pressure in the bulk of the ocean is not separable but a perturbation moves steadily along a slope.

Let's prove that when a quasigeostrophic flow above a uniform slope satisfies two conditions, namely:

$\mathrm{C} 1$ : A perturbation propagates along the isobath with constant velocity $\mathrm{c}$, that is

$$
p=p(x-c t, y, z)=p(\hat{x}, y, z)
$$

$\mathrm{C} 2$ : Both pressure $\mathrm{p}$ and its vertical derivative $\mathrm{p}_{\mathrm{z}}$ at the bottom vanish at infinity:

$$
\mathrm{p} \rightarrow 0, \quad \mathrm{p}_{\mathrm{z}} \rightarrow 0 \quad \text { at } \quad \mathrm{x}^{2}+\mathrm{y}^{2} \rightarrow \infty
$$

then the nonlinear Jacobian term in the bottom boundary condition (3.5) vanishes identically and it is reduced to a simple linear relation

$$
\mathrm{p}+\frac{\mathrm{cf}}{\alpha \mathrm{N}^{2}} \mathrm{p}_{\mathrm{z}}=0
$$

Proof: Using C1 we can rewrite (3.5) as 


$$
\mathrm{p}_{\hat{\mathrm{x}}}+\frac{\mathrm{f}}{\alpha \mathrm{N}^{2}}\left[\mathrm{c} \mathrm{p}_{\mathrm{x} z}+\frac{1}{\rho_{0} \mathrm{f}} \mathrm{J}_{\hat{\mathrm{x}}}\left(\mathrm{p}, \mathrm{p}_{\mathrm{z}}\right)\right]=0, \quad \mathrm{~J}_{\hat{x}}(\mathrm{~A}, \mathrm{~B})=\mathrm{A}_{\hat{\mathrm{x}}} \mathrm{B}_{\mathrm{y}}-\mathrm{A}_{\mathrm{y}} \mathrm{B}_{\hat{\mathrm{x}}}
$$

This last expression can be written in the form:

$$
J_{\hat{x}}\left(p+\rho_{0} c f y, p_{z}-\rho_{0} \alpha N^{2} y\right)=0
$$

From this the functional relationship follows immediately:

$$
p+\rho_{0} c f y=F\left(p_{z}-\rho_{0} \alpha N^{2} y\right)
$$

Using $\mathrm{C} 2$ we can find that $\mathrm{F}$ is a linear function of its argument:

$$
\mathrm{F}(*)=-\frac{\mathrm{cf}}{\alpha \mathrm{N}^{2}}(*)
$$

Therefore for all streamlines we have

$$
p+\rho_{0} c f y=-\frac{c f}{\alpha N^{2}} p_{z}+\rho_{0} c f y
$$

and we immediately obtain (3.6).

This is again similar to the case of a planetary beta-plane and uniform slope topography in a homogeneous ocean. In particularly it shows that in the case of a uniform slope there can be no soliton-like steadily moving solution because nonlinearity is absent. This is also true for a homogeneous ocean as was shown by Malanotte-Rizzoli and Hendershott (1980).

Now suppose that the buoyancy frequency $\mathrm{N}$ is uniform everywhere in the ocean (this approximation is rather good at least for bottom-trapped motion since stratification changes slowly beneath the main thermocline). With the introduction of a stretched vertical coordinate $\widetilde{z}=\mathrm{z} N / \mathrm{f}$ the boundary condition (3.6) becomes:

$$
\mathrm{p}+\frac{\mathrm{c}}{\tilde{\alpha f}} \mathrm{p}_{\tilde{\mathrm{z}}}=0, \quad \tilde{\alpha}=\alpha \mathrm{N} / \mathrm{f}
$$

and the interior pressure field must satisfy a Poisson equation

$$
\Delta \mathrm{p}=\rho_{0} \mathrm{f} \sigma, \quad \Delta=\partial_{\mathrm{xx}}^{2}+\partial_{\mathrm{yy}}^{2}+\partial_{\mathrm{zz}}^{2}
$$

where $\sigma$ is a quasigeostrophic potential vorticity (PV).

At the ocean surface we must add another boundary condition (for example the one used in a rigid-lid approximation): 


$$
\mathrm{p}_{\tilde{\mathrm{z}}}=0 \quad \text { @ } \tilde{\mathrm{z}}=-\tilde{\mathrm{D}}
$$

where $\mathrm{D}$ is the ocean depth at the origin of the coordinate system.

If $\mathrm{PV}$ is not constant then for the solution to be steadily propagating PV must be a function of a moving coordinate $\hat{x}$ that is $\sigma=\sigma(\mathrm{x}-\mathrm{ct}, \mathrm{y}, \widetilde{\mathrm{z}})$ which means that surfaces of constant $\sigma$ must move with the uniform velocity $\mathrm{c}$ along the slope.

\subsection{Formulation of the problem for a steadily translating point vortex}

Now suppose that the potential vorticity is singular in the interior and has a form

$$
\sigma=4 \pi \mathrm{I} \delta\left(\mathrm{x}-\mathrm{ct}, \mathrm{y}^{\prime}, \tilde{\mathrm{z}}^{\prime}+\widetilde{\mathrm{H}}\right)
$$

This means that a three-dimensional point vortex moves steadily above the slope, I - its "strength". The "strength" is a three-dimensional equivalent of a circulation and has a meaning of the product of a potential vorticity anomaly and its volume: $\mathrm{I}=\sigma_{0} \mathrm{~V}$, I is kept constant as $\mathrm{V}$ goes to zero. We shall introduce another coordinate system $\left(\hat{\mathrm{x}}, \mathrm{y}^{\prime}, \widetilde{\mathrm{z}}^{\prime}\right)$, which is turned at the angle $\tilde{\alpha}$ to the original system $(\hat{x}, y, \tilde{z})$ (see figure 3.2 ), $y^{\prime}$ - shoreward along the bottom. The vortex is placed so that a perpendicular line drawn from it to the bottom (in a "stretched" geometry) goes through the origin of the coordinate system (at a given time moment). $\mathrm{H}$ is the distance between the vortex and the bottom, $\widetilde{\mathrm{H}}=\mathrm{HN} / \mathrm{f}$. The new and the old coordinate systems are related as follows:

$$
\begin{array}{ll}
\mathrm{y}^{\prime}=\mathrm{y} \cos \tilde{\alpha}-\tilde{\mathrm{z}} \sin \tilde{\alpha} & \mathrm{p}_{\tilde{\mathrm{z}}}=\cos \tilde{\alpha} \mathrm{p}_{\tilde{\mathrm{z}}^{\prime}}-\sin \tilde{\alpha} \mathrm{p}_{\mathrm{y}^{\prime}} \\
\tilde{\mathrm{z}}^{\prime}=\mathrm{y} \sin \tilde{\alpha}+\tilde{\mathrm{z}} \cos \tilde{\alpha} & \mathrm{p}_{\mathrm{y}}=\cos \tilde{\alpha} \mathrm{p}_{\mathrm{y}^{\prime}}+\sin \tilde{\alpha} \mathrm{p}_{\mathrm{z}^{\prime}}
\end{array}
$$

In our approximation $\tilde{\alpha} \ll 1$ then $\cos \tilde{\alpha} \approx 1, \quad \sin \tilde{\alpha} \approx \tilde{\alpha}$ and we can rewrite (3.9) as: 
surface

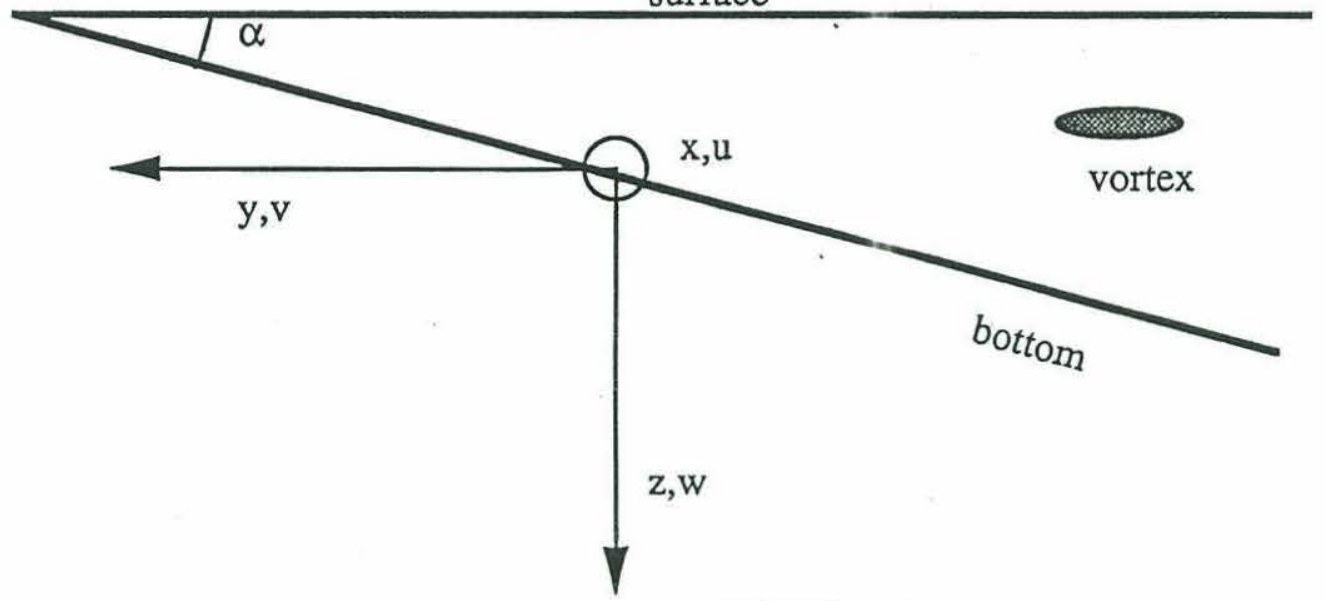

Figure 3.1. A baroclinic vortex above a sloping bottom.

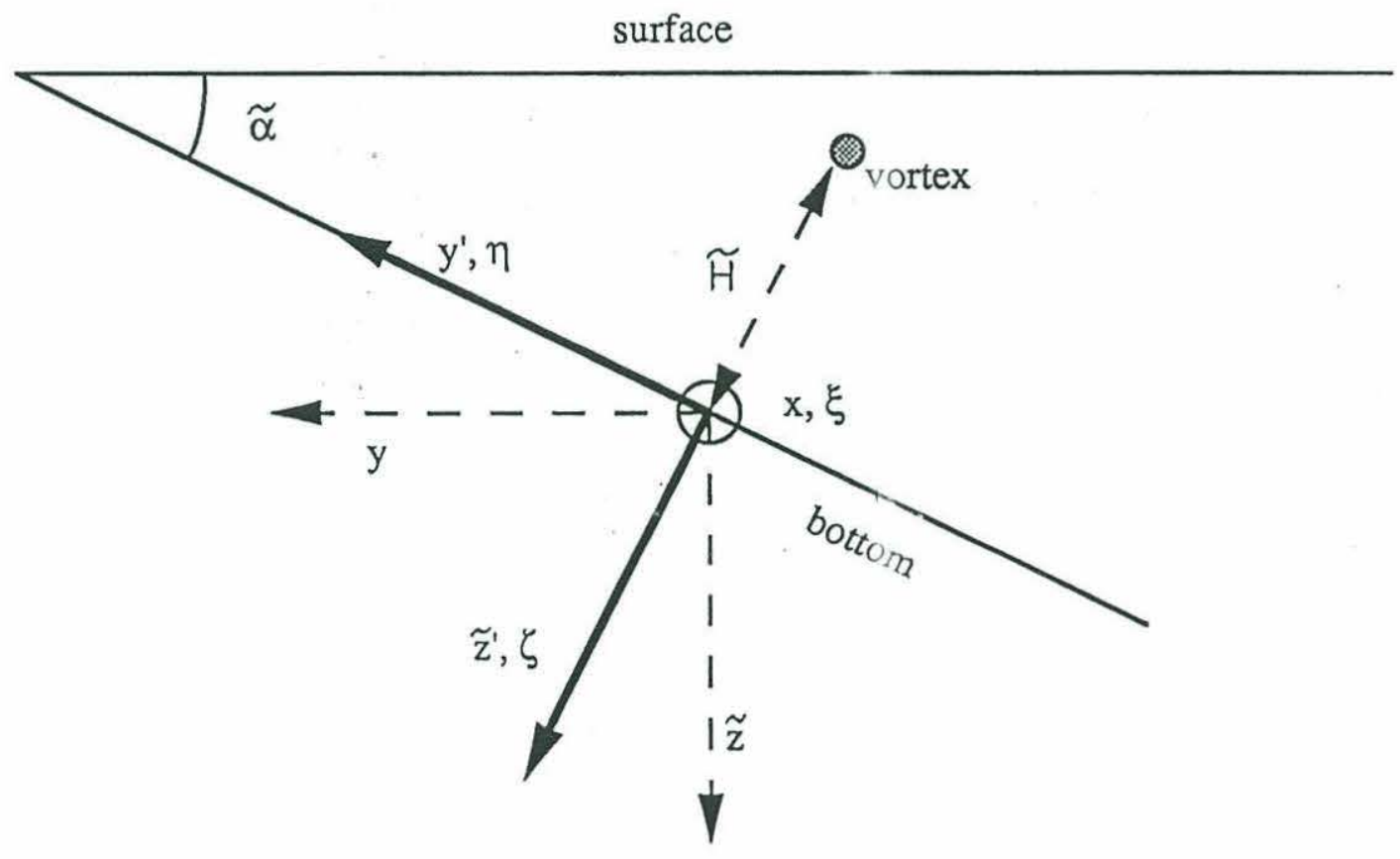

Figure 3.2 The coordinate systems $(\mathrm{x}, \mathrm{y}, \tilde{\mathrm{z}})$ and $\left(\mathrm{x}, \mathrm{y}^{\prime}, \tilde{\mathrm{z}}^{\prime}\right)$ used for the solution. Vortex is at the point $(0,0,-\widetilde{H})$ in the primed, (tilted) coordinate system. 


$$
\begin{array}{ll}
\mathrm{y}^{\prime}=\mathrm{y}-\tilde{\alpha} \tilde{\mathrm{z}} & \mathrm{p}_{\mathrm{z}}=\mathrm{p}_{\tilde{\mathrm{z}}^{\prime}}-\tilde{\alpha} \mathrm{p}_{\mathrm{y}^{\prime}} \\
\tilde{\mathrm{z}}^{\prime}=\tilde{\alpha} \mathrm{y}+\tilde{\mathrm{z}} & \mathrm{p}_{\mathrm{y}}=\mathrm{p}_{\mathrm{y}^{\prime}}+\tilde{\alpha} \mathrm{p}_{\tilde{\mathrm{z}}^{\prime}}
\end{array}
$$

Because the Laplacian operator is spherically symmetric the interior equation will not change when the coordinate system is turned. The velocity $\mathrm{c}$ of the vortex must be determined from the solution itself :

$$
\mathrm{c}=-\frac{1}{\rho_{0} \mathrm{f}} \mathrm{p}_{\mathrm{y}} \quad \text { at the position of a vortex }
$$

that is vortex moves geostrophically due to the "response" from the bottom.

Let's introduce nondimensional coordinates $\tau, \xi, \eta^{\prime}, \zeta^{\prime}$ and pressure (streamfunction) $\Psi:$

$$
\mathrm{t}=\tau \widetilde{\mathrm{H}} / \mathrm{c}, \mathrm{y}^{\prime}=\widetilde{\mathrm{H}} \eta^{\prime}, \mathrm{x}=\widetilde{\mathrm{H}} \xi, \widetilde{\mathrm{z}}^{\prime}=\widetilde{\mathrm{H}} \zeta^{\prime}, \quad \mathrm{p}=\rho_{0} \mathrm{f}^{-1} \mathrm{I} \Psi, \quad \widetilde{\mathrm{D}}=\widetilde{\mathrm{H}} \mathrm{d}
$$

We can express our variables and operators in terms of these nondimensional coordinates:

$$
\begin{gathered}
\mathrm{c}=-\frac{\mathrm{I}}{\widetilde{\mathrm{H}}^{2}}\left(\Psi_{\eta^{\prime}}+\widetilde{\alpha} \Psi_{\zeta^{\prime}}\right), \\
\Delta \Psi\left(\xi, \eta^{\prime}, \zeta^{\prime}\right)=\frac{1}{\rho_{0} \mathrm{f} \widetilde{\mathrm{H}}^{2}} \Delta \mathrm{p}(\mathrm{x}, \mathrm{y}, \tilde{\mathrm{z}}), \quad \delta\left(\xi, \eta^{\prime}, \zeta^{\prime}\right)=\widetilde{\mathrm{H}}^{3} \delta(\mathrm{x}, \mathrm{y}, \widetilde{\mathrm{z}})
\end{gathered}
$$

(The last relation is valid because the Dirac delta-function has an inverse dimension of a unit volume which in our case is $\widetilde{\mathrm{H}}^{3}$ ).

Combining the interior equation (3.7), the boundary conditions (3.6'), (3.8) and the relation (3.10) we can now explicitly formulate the problem in terms of a pressure perturbation $\Psi$ :

$$
\begin{aligned}
& \Delta \Psi=4 \pi \delta\left(\xi-\tau, \eta^{\prime}, \zeta^{\prime}+1\right), \quad \Delta=\partial_{\xi \xi}^{2}+\partial_{\eta^{\prime} \eta^{\prime}}^{2}+\partial_{\zeta^{\prime} \zeta^{\prime}}^{2} \\
& \begin{array}{lll} 
& \text { in the domain }-\mathrm{d}+\tilde{\alpha} \eta^{\prime}<\zeta^{\prime}<0 \\
\Psi+\mathrm{S} \Psi_{\zeta^{\prime}}=0 & \text { @ } \zeta^{\prime}=0 & \text { (bottom) } \\
\Psi_{\zeta^{\prime}}=0 & \text { @ } \zeta^{\prime}=-\mathrm{d} & \text { (surface) }
\end{array}
\end{aligned}
$$




$$
\mathrm{S} \equiv \frac{\mathrm{cf}}{\alpha \mathrm{N}^{2} \mathrm{H}}=\frac{\mathrm{c}}{\widetilde{\alpha} \mathrm{f} \widetilde{H}}=-\frac{\mathrm{A}}{\widetilde{\alpha}}\left(\Psi_{\eta^{\prime}}+\widetilde{\alpha}_{\zeta^{\prime}}\right) \quad @ \quad(\xi=\tau, 0,-1),
$$

where

$$
\mathrm{A}=\mathrm{I} / \mathrm{f} \widetilde{\mathrm{H}}^{3}
$$

is a nondimensional "strength" of a vortex.

The problem (3.11a-c) appears to be a linear elliptic problem with Neuman-type boundary conditions. But the parameter $\mathrm{S}$ in the boundary condition (3.11b) depends on the amplitude and the structure of the solution itself. It plays the role of an eigenvalue and must be found together with the streamfunction $\Psi$.

If the surface and the bottom are absent then a nondimensional pressure perturbation from the point vortex will be

$$
\Psi=-\frac{1}{r}, \quad r^{2}=(\xi-\tau)^{2}+\eta^{\prime 2}+\zeta^{\prime 2}
$$

It is also convenient to nondimensionalize the translational velocity $\mathrm{c}$ which we do not know a priori in terms of the velocity $\mathrm{u}^{0}$ which the vortex induces at the origin of the coordinate system but without the influence of the bottom:

$$
v=\mathrm{c} / \mathrm{u}^{0}
$$

( $v$ - nondimensional). This velocity $\quad u^{0}$ can easily be found:

$$
\mathrm{u}^{0}=-\frac{\tilde{\mathrm{\alpha}}}{\widetilde{\mathrm{H}}^{2}}=-\frac{\text { If } \alpha}{\mathrm{NH}^{2}}
$$

The parameter $\mathrm{S}$ in the system (3.11) has a simple physical meaning: it corresponds approximately to the ratio of an isopycnal inclination angle to the slope angle $\alpha$. Indeed, from the thermal wind relation

$$
\mathrm{u}_{\mathrm{z}}=\frac{\mathrm{g}}{\rho_{0} \mathrm{f}} \rho_{\mathrm{y}}
$$

For the angle of isopycnal inclination $\gamma$ we have: 


$$
\gamma=-\frac{\rho_{\mathrm{y}}}{\rho_{\mathrm{z}}} \approx-\frac{\rho_{\mathrm{y}}}{\rho_{0 \mathrm{z}}}=\frac{\mathrm{f} \rho_{0}}{\mathrm{~g}} \mathrm{u}_{\mathrm{z}} / \frac{\rho_{0}}{\mathrm{~g}} \mathrm{~N}^{2}=\frac{\mathrm{f}}{\mathrm{N}^{2}} \mathrm{u}_{\mathrm{z}}
$$

Estimating $\mathrm{u}_{\mathrm{z}}$ as $\mathrm{c} / \mathrm{H}$ we have $\gamma \approx \frac{\mathrm{f} c}{\mathrm{~N}^{2} \mathrm{H}}$ and thus the ratio of $\gamma$ and $\alpha$ is approximately equal to parameter $\mathrm{S}$ :

$$
\frac{\gamma}{\alpha} \approx \frac{\mathrm{cf}}{\alpha \mathrm{N}^{2} \mathrm{H}}=\mathrm{S}
$$

If $\mathrm{S}>0$ then isopycnal surfaces are inclined in the same direction as the bottom and there is a positive density anomaly at the bottom under a vortex. If $S \geq 1$ then isopycnals in the region between the vortex and the bottom are approximately parallel to the bottom and if $S>>1$ then they can be even steeper than the bottom itself. For $c=8 \mathrm{~cm} / \mathrm{s}, \mathrm{f}=10^{-4} \mathrm{~s}^{-1}, \mathrm{~N}=2 \cdot 10^{-3} \mathrm{~s}^{-1}, \alpha=2 \cdot 10^{-3}$, $\mathrm{H}=1 \mathrm{~km}$ the parameter $\mathrm{S}$ is equal to unity $\mathrm{S}=1$. This estimate shows that the parameter $\mathrm{S}$ is of the order $\mathrm{O}(1)$ and is unlikely to be much more than unity.

\subsection{The solution for the limiting cases}

At first we consider two limiting cases, when $|S|<<1$ and $|S|>>1$. Suppose that the surface is far above the vortex so that $d \gg 1$ and its influence on the dynamics is negligible. Then the problem is equivalent to that of an electrostatic potential of a point charge in the semi-infinite space and to solve it we can exploit the image method developed in electrostatics. The solution will depend on the type of a boundary condition:

1). $\underline{|S| \rightarrow 0}$.

Then (3.11b) becomes

$$
\Psi=0 \quad \text { at } \quad \zeta^{\prime}=0
$$

In order to satisfy this we must put an "image" vortex at $\mathbf{r}=(0,0,1)$ (mirror symmetrical of the real vortex relative to the bottom, figure 3.3a) of the same strength but of the opposite sign. Thus the real and image vortices form a tilted dipole (heton). Because the azimuthal velocity in each direction from the vortex decreases as an inverse square of the distance one can obtain that 


$$
v_{|S| \rightarrow 0}=1 / 4
$$

that is the translational velocity is one quarter of the velocity induced by a real vortex at the origin of coordinate system and in the same direction as that velocity.

2) $\underline{|S| \rightarrow \infty}$.

Now because $\tilde{\alpha}<<1$ (3.11b) becomes

$$
\Psi_{\zeta^{\prime}}=0 \quad \text { at } \zeta^{\prime}=0
$$

To satisfy (3.15) we must put a mirror symmetrical image vortex of the same sign and the same strength. Now

$$
v_{|S| \rightarrow \infty}=-1 / 4
$$

and the vortex will propagate in the opposite direction. Although the parameter $\mathrm{S}$ is large in this case the velocity $\mathrm{c}$ will be small because as $\tilde{\alpha}$ goes to zero the velocity scale $\mathrm{u}_{0}$ will tend to zero too.

Summarizing these extreme cases it can be said that when a vortex is relatively weak or far from the bottom and the bottom is rather steep (ISI $<<1)$ a cyclonic vortex (I>0) should go with shallower water on its right side and an anticyclonic one - on the left side. If a vortex is strong, near the bottom which is not steep (ISI >> 1) then cyclonic one should go to the right of the upslope direction and anticyclonic - to the left of it.

These limits have apparent physical meaning: when $|S|<<1$ isopycnals are only slightly disturbed and intersect the bottom almost on the same line as in unperturbed state. Then role of the bottom is similar to the role of the vertical wall and the boundary condition at this wall again invokes the opposite-signed image vortex. When isopycnals are approximately parallel to the bottom then the boundary condition is more similar to that of on the flat bottom and to satisfy it the image vortex of the same sign is needed. Because it will be slightly tilted it will induce a steady translation which is not the case if the bottom is strictly horizontal. 


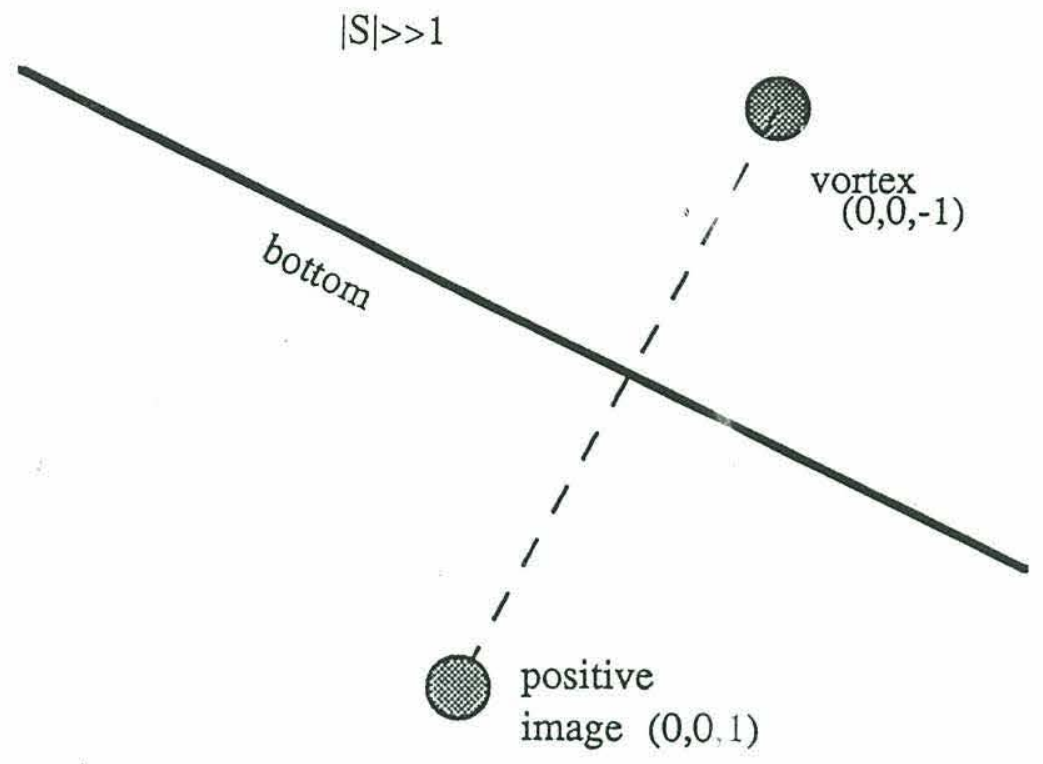

b)

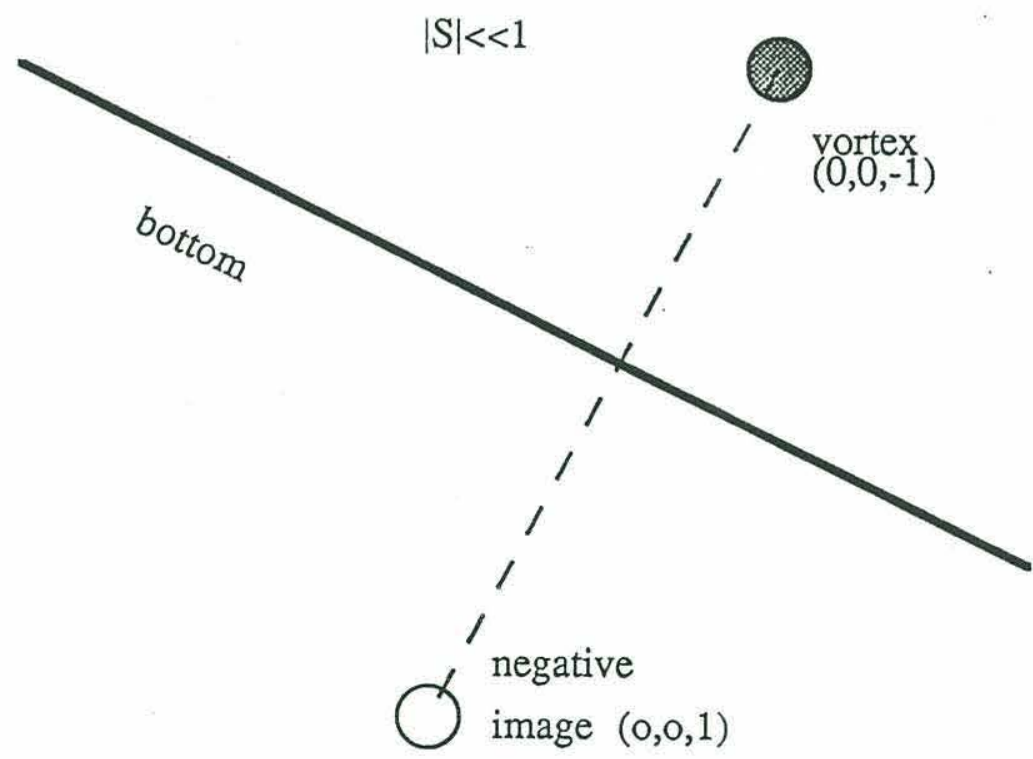

a)

Figure 3.3 (a,b) Real and image vortices when $\mathrm{S} \rightarrow 0$ (a) and $\mathrm{S} \rightarrow \infty$ (b). 

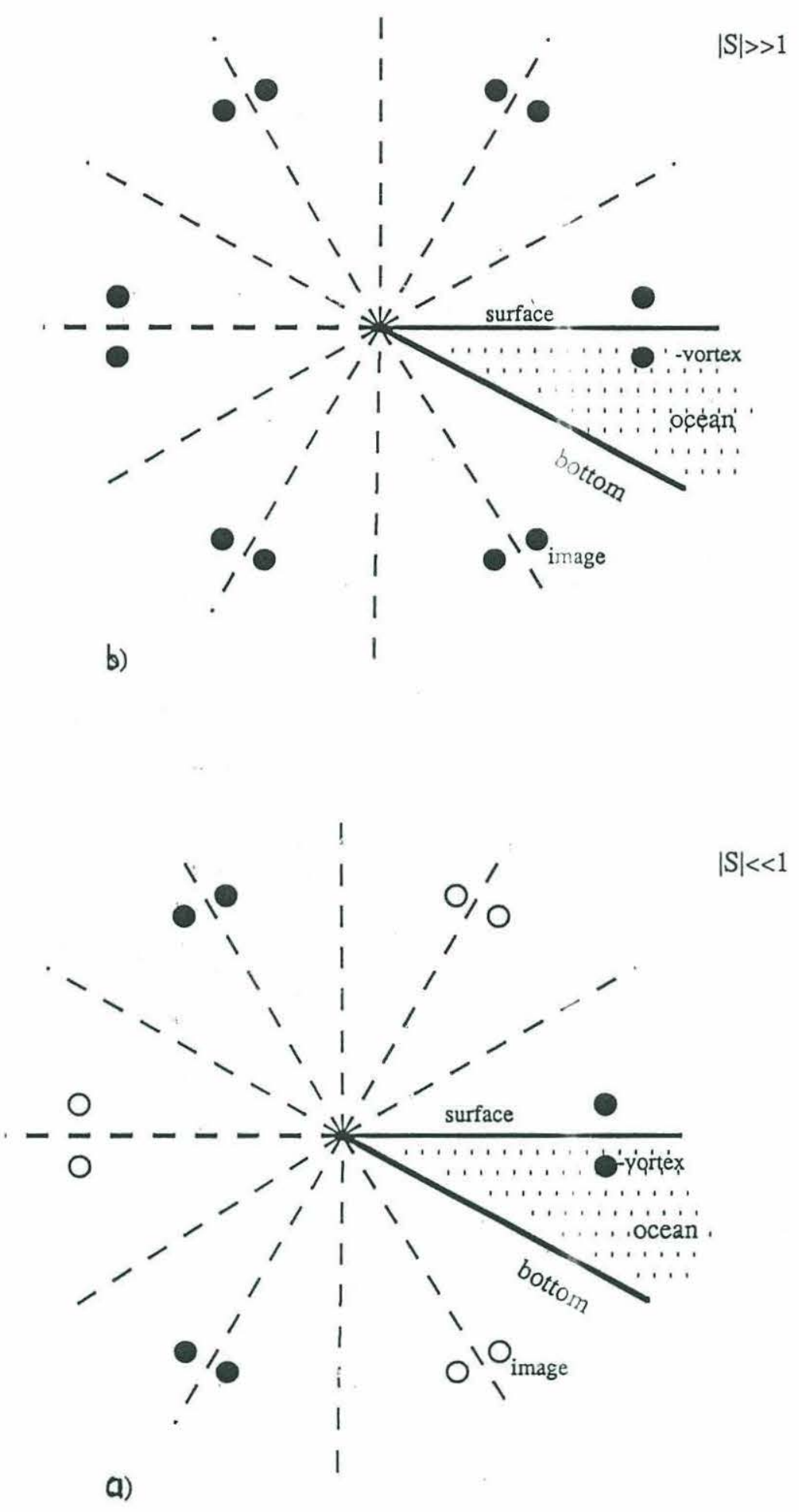

Figure 3.4 System of images when the surface is present.
(a) $\mathrm{S} \rightarrow 0$,
(b) $\mathrm{S} \rightarrow \infty$. 


\section{3) Presence of the surface.}

If a vortex is near the surface then we can not neglect its influence. But again in these limiting cases the problem has an electrostatic analog: to satisfy the surface boundary condition (3.11c) we can construct a system of images as shown in figure 3a,b which is the result of successive reflections of the system from the bottom and the surface. For the case $|\mathrm{S}| \gg>1$ all images have the same sign while for $|S| \ll<1$ pairs of images have alternating signs. This can be done rigorously only when $\tilde{\alpha}=\pi / 2 n$ where $n$ is integer but if angle of the slope is small then quantization is not important. From purely geometrical considerations it can be shown that the parameter $v$ will be a sum of the velocities induced by all these images:

$$
v=\frac{\sin \tilde{\alpha}}{4 d^{2}} \sum_{i=1}^{n}(-1)^{L_{0, \infty}}\left(\frac{\sin \vartheta_{i}^{a+}}{\sin ^{2} \tau_{i}^{a+}}+\frac{\sin \vartheta_{i}^{a-}}{\sin ^{2} \tau_{i}^{a-}}+\frac{\sin \vartheta_{i}^{b+}}{\sin ^{2} \tau_{i}^{b+}}+\frac{\sin \vartheta_{i}^{b-}}{\sin ^{2} \tau_{i}^{b-}}\right)
$$

Here $\mathrm{L}_{0}=\mathrm{i}$ (images of alternating signs) and $\mathrm{L}_{\infty}=0$ (of the same sign). Each number $\mathrm{i}$ in the sum contains the quartet of image vortices designated by a+, a-, b+, b- (a -for "above", b for "below", + and - for positions symmetric to the lines obtained by reflection of the surface and the bottom from each other). In this sum $\vartheta_{i}$ is the angle from the line connecting the real and the image vortices to the vertical, $\tau_{\mathrm{i}}$ - half of the angle between the directions from the edge to the image and real vortices. It can be shown after some calculations that

$$
\left[\begin{array}{c}
\vartheta_{\mathrm{i}}^{\mathrm{a}+} \\
\vartheta_{\mathrm{i}}^{\mathrm{a}-} \\
\vartheta_{\mathrm{i}}^{\mathrm{b}+} \\
\vartheta_{\mathrm{i}}^{\mathrm{b}-}
\end{array}\right]=\tilde{\alpha}\left[\begin{array}{c}
\mathrm{i} \\
\mathrm{i}-\mathrm{q} \\
\mathrm{i}+\mathrm{q} \\
\mathrm{i}
\end{array}\right], \quad\left[\begin{array}{c}
\tau_{\mathrm{i}}^{\mathrm{a}+} \\
\tau_{\mathrm{i}}^{\mathrm{a}-} \\
\tau_{\mathrm{i}}^{\mathrm{b}+} \\
\tau_{\mathrm{i}}^{\mathrm{b}-}
\end{array}\right]=\tilde{\alpha}\left[\begin{array}{c}
\mathrm{i}-\mathrm{q} \\
\mathrm{i} \\
\mathrm{i} \\
\mathrm{i}+\mathrm{q}
\end{array}\right]
$$

where $\mathrm{q}=1-1 / \mathrm{d}$ is a fraction of a distance from the vortex to the surface relative to the total depth of the ocean. When the vortex is at the surface then $\mathrm{q}=0$ and all the above described angles are equal to $\tilde{\alpha i}$.

Results of calculation of the sum (3.16) are shown in figure 3.5a,b. Parameter $v$ is plotted depending on the angle $\tilde{\alpha}$ for several values of $\mathrm{d}$ - ratio of the total depth to the depth under the vortex. One can see that when $|\mathrm{S}|<<1$ (fig. 3.5a) the translation velocity increases when the vortex is between the surface and the bottom. When the vortex is at the surface it can rise up to about 3 times $(v \approx .70)$. When $|S| \gg 1$ (fig. 3.5b) parameter $v$ can increase many times. 

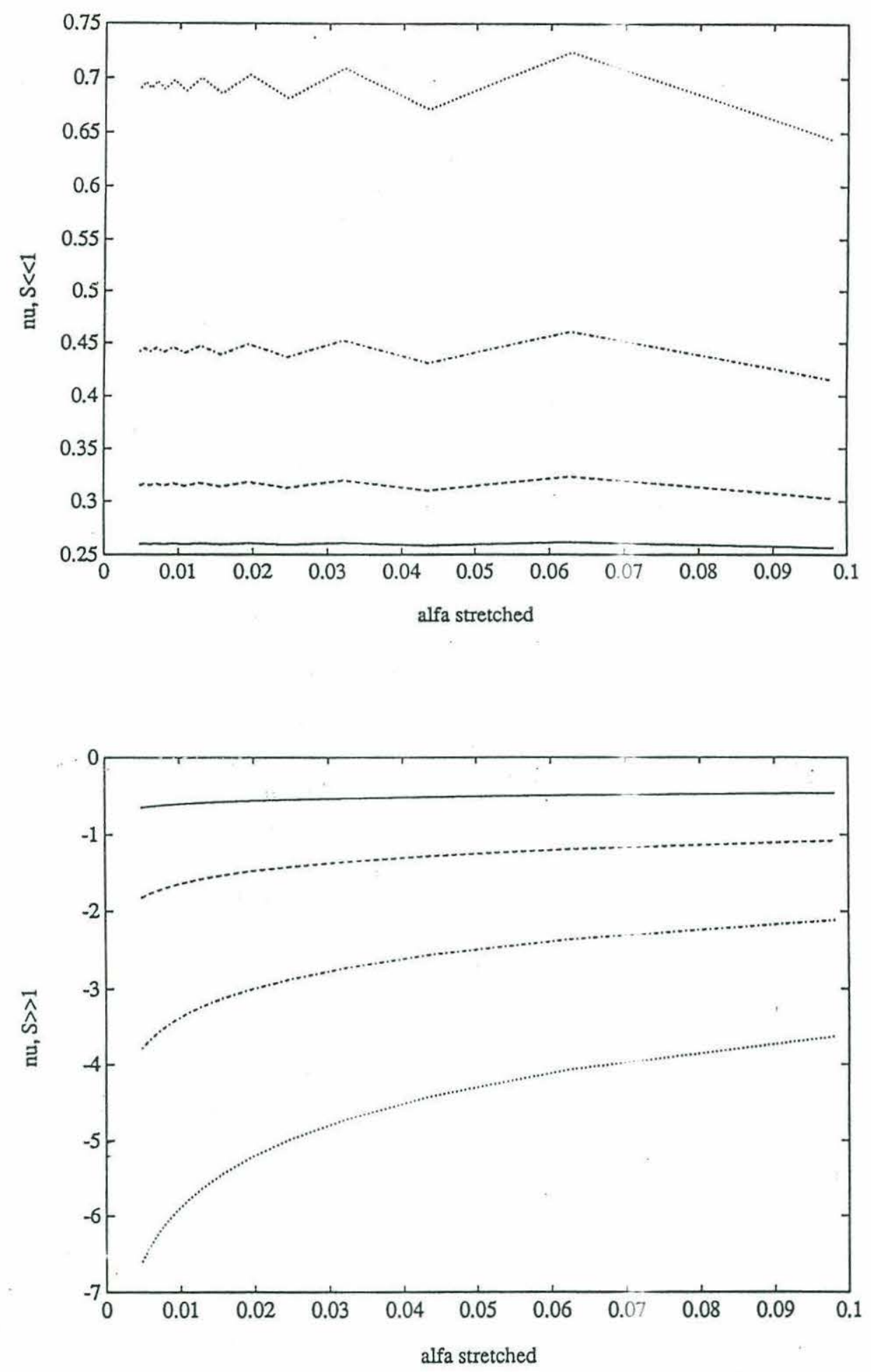

Figure 3.5 Dependence of the parameter $v$ (non-dimensional velocity) on the angle $\tilde{\alpha}$ for different depths $d$ of the vortex.

(a) $\mathrm{S} \rightarrow 0$, (b) $\mathrm{S} \rightarrow \infty$.

Solid line $-\mathrm{d}=4$, dashed $-\mathrm{d}=2$, dot-dashed $-\mathrm{d}=1.33$, dotted $-\mathrm{d}=1$. 
Actually $v$ goes to infinity when $\tilde{\alpha}$ tends to zero at a constant finite value of $\mathrm{d}$ is finite. It does not however imply infinite velocities because the velocity scale $\mathrm{u}^{0}$ goes to zero even faster at this limit.

Thus the translational velocity can increase dramatically in the case $|\mathrm{S}|>>1$ (all the vortices are of the same sign) and only slightly when $|\mathrm{S}|<<1 \quad$ (alternating signs of image vortices).

\subsection{The solution for arbitrary values of the parameter $\mathrm{S}$}

\section{1) Without the surface.}

When $\mathrm{S}=\mathrm{O}(1)$ the simple constructions described in the previous section are not valid. We have to resort to a probably less elegant but more universal method. We assume again at first that the surface is infinitely distant above the vortex. The demonstration of the solution method is less tedious in this case and the presence of a surface also creates some additional difficulties which we shall discuss later. Let's try to seek the solution of (3.11) (the part of it corresponding to the "response" of the bottom to the presence of the vortex) as a Fourier-Bessel integral which is a superposition of bottom-trapped separable radially symmetric harmonics:

$$
\Psi^{(\mathrm{r})}=\int_{0}^{\infty} \Psi_{\mu}^{(\mathrm{r})} \mathrm{J}_{0}(\mu \mathrm{r}) \exp \left(\mu \zeta^{\prime}\right) d \mu
$$

Each of the harmonics $\Psi_{\mu}^{(\mathrm{r})}$ in this expansion satisfies the Laplacian equation $\Delta \Psi^{(\mathrm{r})}=0$ but does not generally satisfy the bottom boundary condition $4.11 \mathrm{~b}$ (except for the harmonic with $\mu=-S^{-1}$; this is the case of free quasigeostrophic topographic waves). In this boundary condition 4.11b we neglect the term $\tilde{\alpha} S \Psi_{\eta^{\prime}}$ proportional to $\tilde{\alpha}$ which we shall assume to be small (this is needed anyway for the validity of the quasigeostrophic approximation). Other harmonics in (3.17) do not satisfy the dispersion relation. Their sum in the bottom boundary condition must compensate for the term $\Psi^{(v)}+\mathrm{S}_{\zeta}{ }_{\zeta}^{(v)}$ of a vortex. To do this we shall expand the field induced by the vortex at the bottom in a similar Fourier-Bessel integral: 


$$
\Psi^{(\mathrm{v})}+\mathrm{S} \Psi_{\zeta^{\prime}}^{(\mathrm{v})}=-\frac{1}{\mathrm{r}_{\mathrm{b}}}+\mathrm{S} \frac{1}{\mathrm{r}_{\mathrm{b}}^{3}}=\int_{0}^{\infty}\left(-\mathrm{F}_{\mu}^{(1)}+\mathrm{SF}_{\mu}^{(2)}\right) \mathrm{J}_{0}\left(\mu \mathrm{r}_{\mathrm{b}}\right) d \mu, \quad \mathrm{r}_{\mathrm{b}}^{2}=1+\xi^{2}+\eta^{\prime 2}
$$

(For simplicity we shall write simply $\xi$ instead of $\xi-\tau$ for the position of a vortex, so its nondimensional coordinates are $(0,0,-1))$.

In this expression the Fourier-Bessel coefficients $\mathrm{F}_{\mu}^{(1)}, \mathrm{F}_{\mu}^{(2)}$ are equal to

$$
\mathrm{F}_{\mu}^{(1)}=\int_{0}^{\infty} \frac{\mathrm{x}}{\left(1+\mathrm{x}^{2}\right)^{1 / 2}} \mathrm{~J}_{0}(\mu \mathrm{x}) d \mathrm{x}, \quad \mathrm{F}_{\mu}^{(2)}=\int_{0}^{\infty} \frac{\mathrm{x}}{\left(1+\mathrm{x}^{2}\right)^{3 / 2}} \mathrm{~J}_{0}(\mu \mathrm{x}) d \mathrm{x}
$$

The "response" expansion plugged into the boundary condition (3.11b) gives

$$
\Psi^{(\mathrm{r})}+\mathrm{S} \Psi_{\zeta}^{(\mathrm{r})}=\int_{0}^{\infty}(1+\mu \mathrm{S}) \Psi_{\mu}^{(\mathrm{r})} \mathrm{J}_{0}(\mu \mathrm{r}) d \mu \quad @ \quad \zeta^{\prime}=0
$$

Comparing coefficients at each harmonic in (3.18) and (3.20) we obtain:

$$
\Psi_{\mu}^{(\mathrm{r})}=\frac{1}{1+\mu \mathrm{S}}\left(-\mathrm{F}_{\mu}^{(1)}+\mathrm{SF}_{\mu}^{(2)}\right)
$$

After performing the Fourier expansion (3.18) we shall be able to describe the perturbation field completely due to the relation (3.21). But the parameter $\mathrm{S}$ in (3.21) must be found from the solution itself. From (3.11e), (3.11d) and (3.13) we have

$$
S=-v A
$$

and also from (3.11d)

$$
\mathrm{S}=-\mathrm{A} \Psi_{\zeta^{\prime}}^{(\mathrm{r})} \quad @ \mathbf{x}=(0,0,-1)
$$

because the solution is $\eta$-symmetric and the other term in (3.11d) vanishes. Substituting (3.21) into (3.17) and then (3.17) into (3.22) we obtain a transcendent (integral) equation on the nondimensional parameter $v$ : 


$$
v=-\int_{0}^{\infty} \frac{\mu}{1-\mu \nu \mathrm{A}} \exp (-\mu)\left[\mathrm{vAF}_{\mu}^{(2)}+\mathrm{F}_{\mu}^{(1)}\right] d \mu
$$

When $\quad \mathrm{VA}>0$ the principle value of this integral is meant; this gives the correct result in the limiting cases of $\mathrm{S} \rightarrow 0$ and $\mathrm{S} \rightarrow \infty$.

Finally using (3.19) we shall rewrite the equation (3.23) for parameter $v$ in the form:

$$
\nu=-\int_{0}^{\infty} \frac{\exp (-\mu)}{(1-\nu A \mu)}\left[\Phi_{\mu}^{(1)}+\nu A \Phi_{\mu}^{(2)}\right] d \mu
$$

where

$$
\Phi_{\mu}^{(i)}=\int_{0}^{\infty} \frac{x}{\left(1+x^{2} / \mu^{2}\right)^{i-1 / 2}} J_{0}(x) d x \quad i=1,2
$$

We know a priori only the parameter A which depends only on the strength of a vortex and its distance from the bottom. The parameter $v$ must be determined from (3.23)-(3.19') which is the transcendent equation. Note however that the right-hand side of this equation depends on the product $v A$ which is the parameter $S$. Thus we can vary $S$ and for each $S$ find the value of the parameter $\mathrm{v}$ and then see what value of $\mathrm{A}$ does this combination of $\mathrm{V}$ and $\mathrm{S}$ correspond to. Figure 3.6(a,b) shows the results of calculation of (3.24) that is the dependence of $v$ on $S$ (fig. 3.6a) and on A (fig. 3.6b, only for moderate values of A). When the ISI goes to infinity the $v$ approaches asymptotically the value -0.25 and when it goes to zero it becomes +0.25 as predicted by the method of image vortices described above. One can also see a strong asymmetry between cyclonic and anticyclonic vortices and between the positive and negative $\mathrm{S}$ at moderate values of this parameter. In general the speed of a vortex is greater at negative $S$ (to the left of onshore direction), that is weak cyclones and strong anticyclones should move faster to this direction than strong cyclones and weak anticyclones to the opposite side. This (negative $S$ ) is the direction of the propagation of free topographic waves that is the "westward" direction analogously to the planetary $\beta$-effect. While for the positive $S$ the parameter $v$ monotonically varies from the one limit to another for the negative $S$ it exhibits two peaks, the first of them (at $S \approx-0.43$ ) is especially distinct. So that although the topographic $\beta$-effect is not a complete analogy to the planetary one it plays certain role in such motions. 


\section{2) Presence of the surface.}

To describe the influence of the surface for an arbitrary value of $S$ it is more convenient to return to the old coordinate system $\mathrm{x}, \mathrm{y}, \tilde{\mathrm{z}}$ (corresponding nondimensional variables are $\xi, \eta, \zeta$ ), except that the origin of coordinate system is strictly under the vortex, so that the vortex is again at the point $(0,0,-1)$ and now $\zeta=-\tilde{\alpha} \eta$ at the bottom. Let's again decompose the nondimensional pressure (or streamfunction) into that of the "vortex" $\Psi^{(\mathrm{v})}$ and the "response" $\Psi^{(\mathrm{r})}$, so that in the interior of the ocean

$$
\Psi^{(\mathrm{r})}=\int_{0}^{\infty}\left(\Psi_{\mu}^{+} \cosh (\mu \zeta)+\Psi_{\mu} \sinh (\mu \zeta)\right) \mathrm{J}_{0}(\mu \mathrm{r}) d \mu
$$

Then at the bottom we have (after the first-order Taylor expansion of functions of $\mu \zeta$ near zero)

$$
\begin{gathered}
\Psi^{(\mathrm{v})}+S \Psi_{\zeta}^{(v)}=-\frac{1}{\mathrm{r}_{\mathrm{b}}}+\frac{\mathrm{S}}{\mathrm{r}_{\mathrm{b}}^{3}}+\left[\frac{3 \mathrm{~S}}{\mathrm{r}_{\mathrm{b}}^{5}}-\frac{(1+\mathrm{S})}{\mathrm{r}_{\mathrm{b}}^{3}}\right] \tilde{\alpha} \eta \\
\Psi^{(\mathrm{r})}+S \Psi_{\zeta}^{(\mathrm{r})}=\int_{0}^{\infty}\left(\left(\Psi_{\mu}^{+}+\mu S \Psi_{\mu}^{-}\right)-\left(\Psi_{\mu}^{-}+\mu S \Psi_{\mu}^{+}\right) \mu \tilde{\alpha} \eta\right) \mathrm{J}_{0}(\mu \mathrm{r}) d \mu
\end{gathered}
$$

At the surface

$$
\begin{gathered}
\Psi_{\zeta}^{(\mathrm{v})}=\frac{1}{(\mathrm{~d}-1)^{2}} \frac{1}{\mathrm{r}_{\mathrm{s}}^{3}}, \quad \mathrm{r}_{\mathrm{s}}^{2}=1+\left(\xi^{2}+\eta^{2}\right) /(\mathrm{d}-1)^{2} \\
\Psi_{\zeta}^{(\mathrm{r})}=\int_{0}^{\infty} \mu\left(-\Psi_{\mu}^{+} \sinh (\mu \mathrm{d})+\Psi_{\mu}^{-} \cosh (\mu \mathrm{d})\right) \mathrm{J}_{0}(\mu \mathrm{r}) d \mu
\end{gathered}
$$

Now we expand the "response" into the powers of $\tilde{\alpha} \eta$ (we shall need only first two terms of this expansion, others will not contribute to the translation velocity) : $\Psi_{\mu}^{ \pm}=\Psi_{\mu}^{0 \pm}+\widetilde{\alpha} \eta \Psi_{\mu}^{1 \pm}+\ldots$. Comparing the coefficients at each harmonic at the surface and the bottom we obtain system of equations analogous to (3.21) when the surface is absent: 


$$
\begin{gathered}
\Psi_{\mu}^{0+}+\mu S \Psi_{\mu}^{0-}=\mathrm{SF}_{\mu}^{(2)}-\mathrm{F}_{\mu}^{(1)} \quad-\Psi_{\mu}^{0+} \sinh (\mu \mathrm{d})+\Psi_{\mu}^{0-} \cosh (\mu \mathrm{d})=\frac{1}{\mu(\mathrm{d}-1)^{2}} \mathrm{~F}_{(\mathrm{d}-1) \mu}^{(2)} \\
\mu \mathrm{S} \Psi_{\mu}^{0+}+\Psi_{\mu}^{0-}-\Psi_{\mu}^{1+}-\mu S \Psi_{\mu}^{1-}=(1+\mathrm{S}) \mathrm{F}_{\mu}^{(2)}-3 \mathrm{SF}_{\mu}^{(3)} \quad-\Psi_{\mu}^{1+} \sinh (\mu \mathrm{d})+\Psi_{\mu}^{1-} \cosh (\mu \mathrm{d})=0
\end{gathered}
$$

Now we can write down the equation for the parameter $v$ :

$$
\begin{aligned}
v=\frac{1}{\widetilde{\alpha}} \frac{\partial \Psi^{(\mathrm{r})}}{\partial \eta} \mathrm{I}_{0,0,-1}= & \int_{0}^{\infty} \frac{\exp (-\mu)}{\mu \mathrm{Z}} \frac{1+\exp [-2 \mu(\mathrm{d}-1)]}{1+\exp [-2 \mu \mathrm{d}]}\left\{(1+\mathrm{S}) \Phi_{\mu}^{(2)}-3 S \Phi_{\mu}^{(3)}+\right. \\
& \left.+\frac{\tanh (\mu \mathrm{d})+\mu \mathrm{S}}{\mathrm{Z}}\left(\Phi_{\mu}^{(1)}-\mathrm{S} \Phi_{\mu}^{(2)}\right)+\frac{1-\mu^{2} \mathrm{~S}^{2}}{\mathrm{Z} \cosh (\mu \mathrm{d}) \mu(\mathrm{d}-1)^{2}} \Phi_{(\mathrm{d}-1) \mu}^{(2)}\right\} \mathrm{d} \mu
\end{aligned}
$$

where $\quad Z=1+\mu S \tanh (\mu d), \quad \Phi_{\mu}^{(i)}$ are the same as in $\left(3.19^{\prime}\right)$.

It can be shown after some algebra that when $d \rightarrow \infty$ this expression becomes equal to (3.24) obtained for semi-infinite ocean. But now we have serious problem when $S<0$. Now in addition to the integrable first-order pole like in (3.24) we have a term with a second order pole which is not integrable (integration gives infinity). This pole naturally corresponds to the dispersion relation for a free topographic Rossby waves. That is we can not rigorously obtain $v$ for negative values of parameter S. Strictly speaking, steady "westward" translation of a vortex in the presence of a surface can not exist. Presence of a barotropic mode therefore makes qualitative changes in the solution. Limit $d \rightarrow \infty$ appears to be a singular one, which is not surprising, since "equivalent barotropic" model is known to be a singular limit of a model with finite depth.

Consider however the numerator $\mathrm{B}(\mu)$ at this second-order pole when the denominator is zero $(S=-1 / \mu \tanh (\mu d))$ :

$$
\begin{aligned}
\mathrm{B}(\mu)=\frac{\exp (-\mu)}{\mu} \frac{1+\exp [-2 \mu(\mathrm{d}-1)]}{1+\exp [-2 \mu \mathrm{d}]}\left\{[\tanh (\mu \mathrm{d})-\operatorname{coth}(\mu \mathrm{d})]\left(\Phi_{\mu}^{(1)}-\mathrm{S} \Phi_{\mu}^{(2)}\right)+\right. & \\
& \left.+\frac{1-\operatorname{coth}(\mu \mathrm{d})}{\cosh (\mu \mathrm{d}) \mu(\mathrm{d}-1)^{2}} \Phi_{(\mathrm{d}-1) \mu}^{(2)}\right\}
\end{aligned}
$$



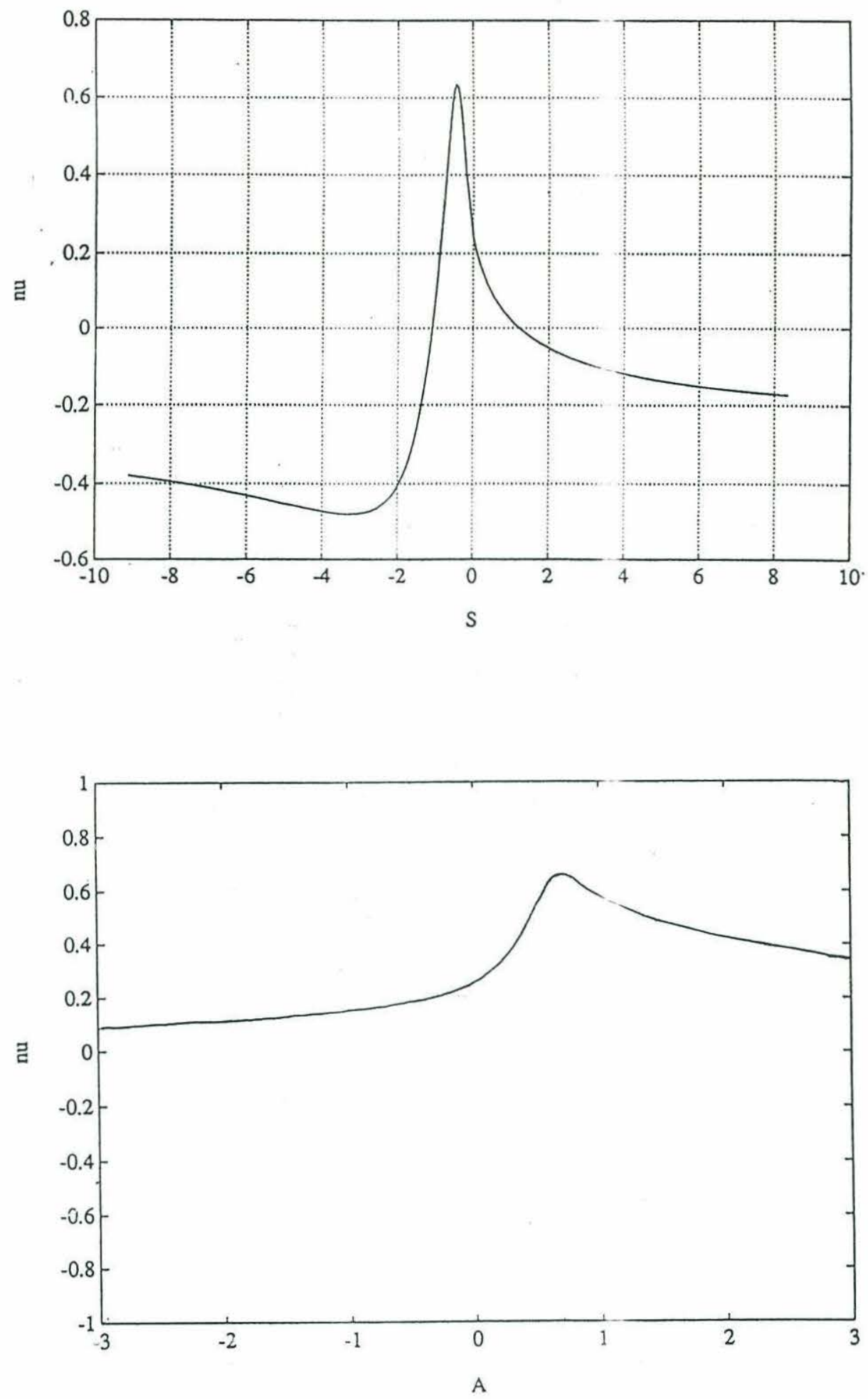

Figure 3.6 (a) Dependence of the parameter $v$ (non-dimensional velocity) on the parameter $\mathrm{S}$ for a semi-infinite ocean.

(b) Dependence of the parameter $\mathrm{v}$ on a nondimensional strength $\mathrm{A}$ of a vortex. 


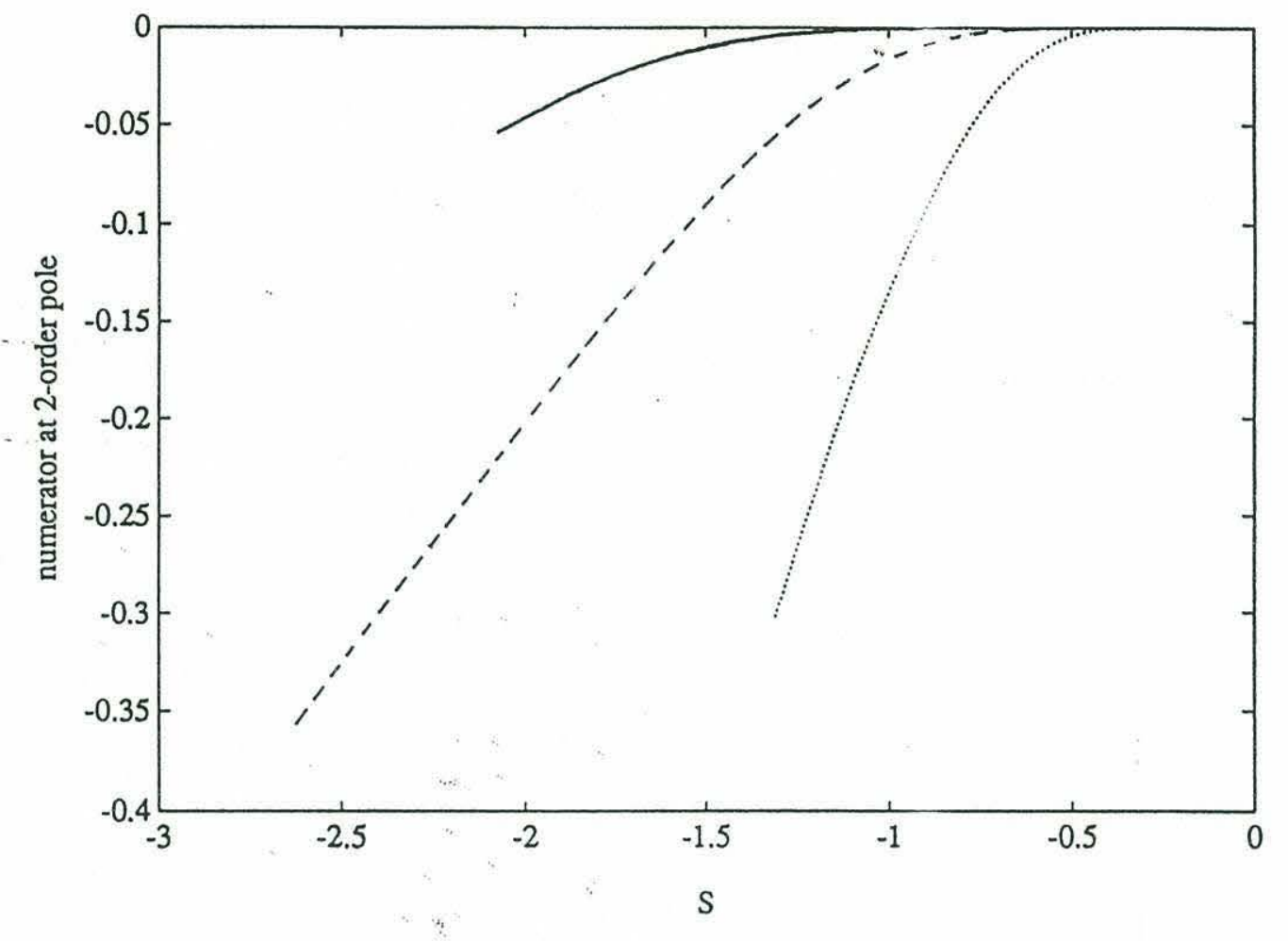

Figure 3.7 Numerator B versus $\mathrm{S}$ at the second-order pole in the equation (3.28): -.-- for $\mathrm{d}=4, \ldots$. . . for $\mathrm{d}=2$ and ..... for $\mathrm{d}=1$ (surface vortex). 

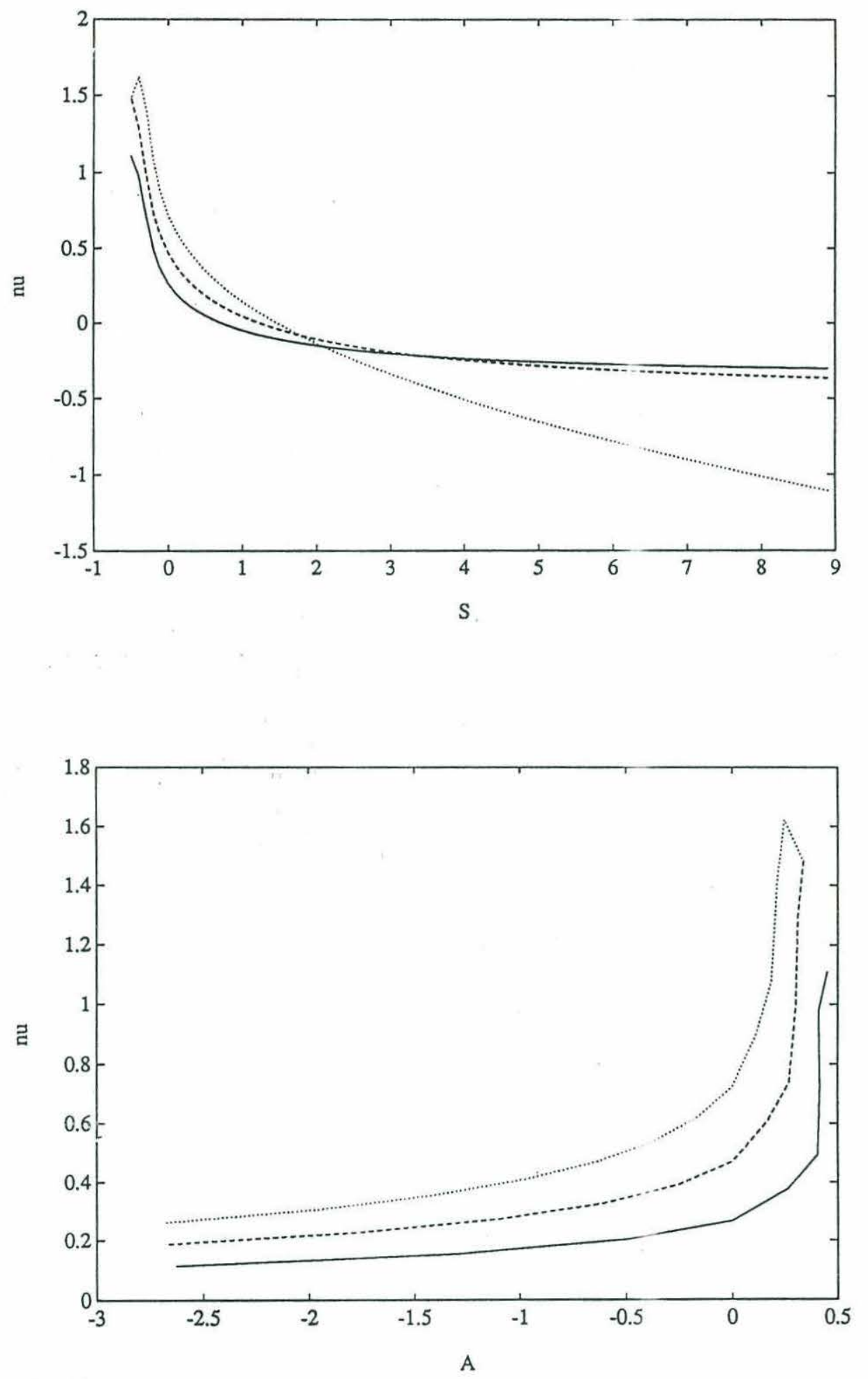

Figure 3.8 (a) Dependence of the parameter $v$ on $S$ for different relative depths of the vortex $d$ :

-.-- for $d=4, \ldots$ for $d=2$ and ..... for $d=1$ (surface vortex).

(b) Dependence of the parameter $v$ on a nondimensional strength $\mathrm{A}$ of a vortex for the same values of $d$. 
In figure 3.7 the function $B$ is plotted against $S$ (that is $-1 / \mu \tanh (\mu \mathrm{d})$ ) for three values of $\mathrm{d}-4,2$ and 1. When $\mathrm{S}$ tends to zero from below this function tends to zero as -exp(-1/S), so that for small $S$ it is almost indistinguishable from zero (it also goes to zero when $S \rightarrow \infty$ which justifies our consideration of this limit in previous section). Thus we can say that only slight change in the structure of the solution is needed to to make $B(S)=0$ for small negative values of $S$. This allows us (though not rigorously) to calculate (3.28) for for this values of $\mathrm{S}$ by simply ignoring the integration around the pole. In fig. $3.8 \mathrm{a}, \mathrm{b}$ results of this integration for $\mathrm{S}$ varying from -.5 to about +9 are shown again for $d=4,2$ and 1 . Previous values of $v$ at $S=0$ are recovered and there is apparently no finite limit of $v$ when $S$ goes to infinity as predicted by the method of images. Qualitatively the behavior of $v$ is similar to that of in semi-infinite ocean. At negative $S$ the parameter $v$ begins to grow but later it must go to minus infinity because of the negative numerator at the pole.

This implies infinite velocities in the direction of the topographic waves propagation (only for anticyclonic vortices). But actually it means that there can not be a steady solution in this case and we must consider transient effects. This can correspond for example to motion of Gulf Stream warm core rings on the continental slope.

Concluding, let's recapitulate how translational velocity of a vortex can be calculated from this theory. We must pick a value of $v$ for known A (dimensionless strength of a vortex) from figure $3.6 \mathrm{~b}$ (or $3.8 \mathrm{~b}$ with the surface). The parameter A can be obtained using (4.11e) if we know the volume of a vortex and its potential vorticity anomaly. For example if we assume that the Gulf Stream warm core rings have a shape of hemiellipsoids of rotation with horizontal semiaxes, say, $r=80 \mathrm{~km}$ and vertical (stretched) semiaxes $\mathrm{h}=500 \mathrm{~m} * 40=20 \mathrm{~km}$, their potential vorticity anomaly is roughly about $-\mathrm{f} / 2$ and depth $\widetilde{\mathrm{H}}=80 \mathrm{~km}$ then $\mathrm{A}=-2 \pi \mathrm{r}^{2} \mathrm{~h} / 3 \widetilde{\mathrm{H}}^{3} \approx-.25$, for the "Shikmona eddy" or Gulf of Mexico rings of the same parameters but stretched depth of $40 \mathrm{~km}$ we obtain $\mathrm{A}=-2$. Warm core rings usually move to the left of the upslope direction (negative $\mathrm{S}$ ). In this case steady translational speed goes to infinity because of the influence of this second-order pole in (3.28) and we can not predict it from this theory.

\subsection{Conclusions}

We have considered self-advection of a baroclinic vortex along a uniformly sloping bottom. Usually it is supposed that the influence of a slope is similar to that of planetary the $\beta$-effect, that is it will cause radiation of topographic Rossby waves and advection of the vortex "westward" - to the left of the upslope direction. Our analysis have shown that this is often not the case and the vortex 
can go in both directions along the slope depending on its strength and the slope angle. Moreover, such translation can be steady and nonradiating: the vortex is advected by a density anomaly at the bottom which is itself created by the vortex velocity field. This is not the case for a monopolar vortex on a planetary $\beta$-plane: vortex will always radiate Rossby waves away unless its total angular momentum is zero. Our study suggests that very strong cyclones over a slope with a small inclination and relatively weak anticyclones over steep slope should move to the right of the upslope while strong anticyclones over gentle slope and weak cyclones over steep slope - "westward" - to the left of the upslope direction. In this ("westward") case the speed of such translation is generally larger which can be interpreted as a manifestation of a topographic $\beta$-effect.

A question arises about the probability of such a dynamical regime. Usually an open mechanical or thermodynamic system tends to a state in which no (or minimum) energy is radiated away, so that steady nonradiating translation can be a state of minimum energy and represent an attractor in phase space of the system. But there are other factors which can cause opposite effects: the amplitude and even the direction of the translation velocity depend strongly on the parameters of the vortex and geometry of the system, so that topography irregularities, ambient flows and waves can distort this picture significantly. One conspicuous feature of the proposed model is that the influence of the surface (the fact that it is not parallel to the bottom) brings about dramatic qualitative changes in the solution. Because of the presence of a barotropic mode in this case it resembles some features of a purely barotropic case, or a planetary beta-plane. In particular "westward" translation is not possible in the steady regime and "eastward" velocity is considerably different from the case of infinitely deep ocean. Even if a vortex is in the mid-depth the translational velocity is much larger than without the surface.

It is interesting to compare the our results with another existing model of a vortex - sloping bottom interaction - Nof's (1983). In his model a patch of water of constant density is lying on the sloping bottom under an infinitely deep and resting upper layer of smaller density. Such vortex can move steadily along the slope with the velocity $\mathrm{C}=\mathrm{g}^{\prime} \alpha / \mathrm{f}$ to the left from the upslope direction, velocity does not depend on the parameters of the vortex (except on $\mathrm{g}^{\prime}$ ). In his model it is obtained as a balance between gravity and coriolis force. In our model, when the vortex is not on the bottom but above it, the balance of forces is much more complicated. It includes the radiation field and can strongly depend on various parameters of the system. Not surprisingly our results show a more complicated possible behavior of a vortex and its interaction with the bottom.

As we already pointed out at the beginning of this chapter the above results are obtained under the assumption that the bottom boundary condition is applied at the actual depth rather than at the reference depth. This appears to be the only factor (in the quasigeostrophic approximation) which breaks the cross-slope symmetry and allows an eddy to move steadily along the slope. 


\section{Chapter 4}

\section{Semi-lagrangian contour dynamics}

\section{for a continuously stratified ocean}

\subsection{Introduction}

In this chapter we shall introduce a numerical technique developed for computation of threedimensional continuously stratified quasi-geostrophic flows over a non-uniform bottom. We shall use it to study the interaction of an upper-ocean eddy with bottom topography, although it is applicable to a considerably broader class of other problems which we shall discuss briefly in the next chapters.

This technique resembles the "contour dynamics" method for two-dimensional vorticity flows, but there are two major differences between them. First - in addition to potential vorticity contours in the interior we use density contours at the bottom (lines where isopycnal surfaces intersect a seafloor). Second - the numerical implementation is quite different from the traditional "contour dynamics" approach. Although we also use positions of density and PV contours explicitly and perform an elliptic inversion using corresponding Green's functions, there are additional procedures of interpolating PV and density fields from moving contours onto a regular grid and velocity fields back from a regular grid to moving contours. These procedures are not performed in the standard "contour dynamics" method. They imply additional computational operations but still make numerics more efficient. In particular they allow a much faster inversion procedure and also a representation of continuous rather than step-like fields.

Usually equations of quasigeostrophic dynamics are written in a potential vorticity -streamfunction form and different methods for solving them rely on the following important properties:

First - a potential vorticity is connected with a streamfunction via an elliptic operator, so a streamfunction and hence a velocity field can be obtained at each timestep inverting a PV distribution. 
This allows splitting the system of equations so that one of them is "static" and does not have a time derivative. This equation is theconnection between a streamfunction and a potential vorticity. The other one is the evolution equation for a PV field. Thus the expensive inversion procedure and the time-stepping are decoupled, which enhances the efficiency of computation.

Second - the evolution equation in QG dynamics is in fact a lagrangian PV conservation property of fluid parcels. This suggests applicability of Lagrangian methods of solving them. In principle we need only to track trajectories of fluid particles with known PV; the velocity field can be obtained as a convolution integral of a potential vorticity distribution with a corresponding Green's function - inverse of the elliptic operator. This implies that the advection velocity field in the evolution equation is a sum of velocities induced by all fluid particles with PV anomalies, trajectories of which we follow. Several lagrangian techniques are based on this property. The simplest example of them is the point vortex method, where the (potential) vorticity is discretized into a finite set of singular anomalies. Recently a more comprehensive "contour dynamics" method was developed (Zabusky et al, 1979) where a convolution integral for a step-like PV distribution is computed along discretized contours rather than over a whole domain.

For a barotropic and a layered baroclinic QG model one needs to know only a potential vorticity distribution in each layer at every timestep. For a 3-dimensional continuously stratified case a PV distribution is not enough. We also need to impose certain boundary conditions at the surface and the bottom of a model. In QG dynamics such boundary conditions can be provided in the form of a density distribution at these boundaries. Since density is related to a vertical derivative of pressure and hence a streamfunction, together with the elliptic operator in the interior we obtain a Neuman-type problem for a streamfunction at each timestep. Since the density is conserved in the adiabatic case, full (evolutionary) boundary conditions are similar to the equation of PV conservation in the interior.

In this work we shall concentrate on the bottom boundary condition for stratified flows over non-uniform bottom topography. In layered QG models topography is represented as a stretching term in PV balance for the lowermost layer. For the continuously stratified case a similar role is played by a density at the bottom which is in some sense equivalent to a PV "sheet" at the bottom and its evolution depends on a topographic elevation. Although this equivalence of a density field and a PV "sheet" at the boundaries was recognized for quite a long time (since Bretherton, 1966) it was hardly used explicitly in numerical models. As we shall use a continuously stratified model, it is necessary to incorporate the bottom boundary condition explicitly. In the method described below we attempt to use this boundary condition in the presence of topography in the density conservation form. Instead of avoiding intersections of isopycnals with topography we are going to use them as primary indicators of the flow-topography interaction - similarly to vorticity contours for twodimensional flows. 


\subsection{The bottom boundary condition as a density conservation equation}

In this section we will focus on the bottom boundary condition based on the (potential) density conservation for fluid parcels, valid for the adiabatic inviscid approximation:

$$
\frac{d \rho}{d t}=\rho_{t}+u \rho_{x}+v \rho_{y}+w \rho_{z}=0
$$

( $\mathrm{u}, \mathrm{v}$ - horizontal velocities, $\mathrm{w}$ - vertical, positive upward).

The idea underlying following derivations is in fact very simple and rely on two facts: first that the density is conserved as water parcel moves along the bottom, and second - that the density is related to the vertical derivative of a pressure field due to the hydrostatic equation. Thus by knowing positions of parcels with the given density at the bottom (isopycnal surfaces) we determine the bottom density field which we can use as a boundary condition to obtain a pressure field and hence velocities everywhere in a domain.

We shall use this property (density conservation) at the bottom where we must satisfy the kinematic boundary condition (no normal flow into the bottom, figure 4.1). This will be written as following:

$$
\mathrm{w}=\mathrm{ub}_{\mathrm{x}}+\mathrm{vb}_{\mathrm{y}} \quad @ \mathrm{z}=\mathrm{b}(\mathrm{x}, \mathrm{y})
$$

We shall also split the density field into the background profile and and the perturbation:

$$
\rho(x, y, z, t)=\rho_{0}(z)+\rho^{\prime}(x, y, z, t)
$$

so that

$$
\rho_{x}=\rho_{x}^{\prime}, \quad \rho_{y}=\rho_{y}^{\prime}, \quad \rho_{z}=\rho_{0 z}+\rho_{z}^{\prime}
$$


ocean with bottom topography

surface $\mathrm{z}=\mathrm{H}$

$N(z)$

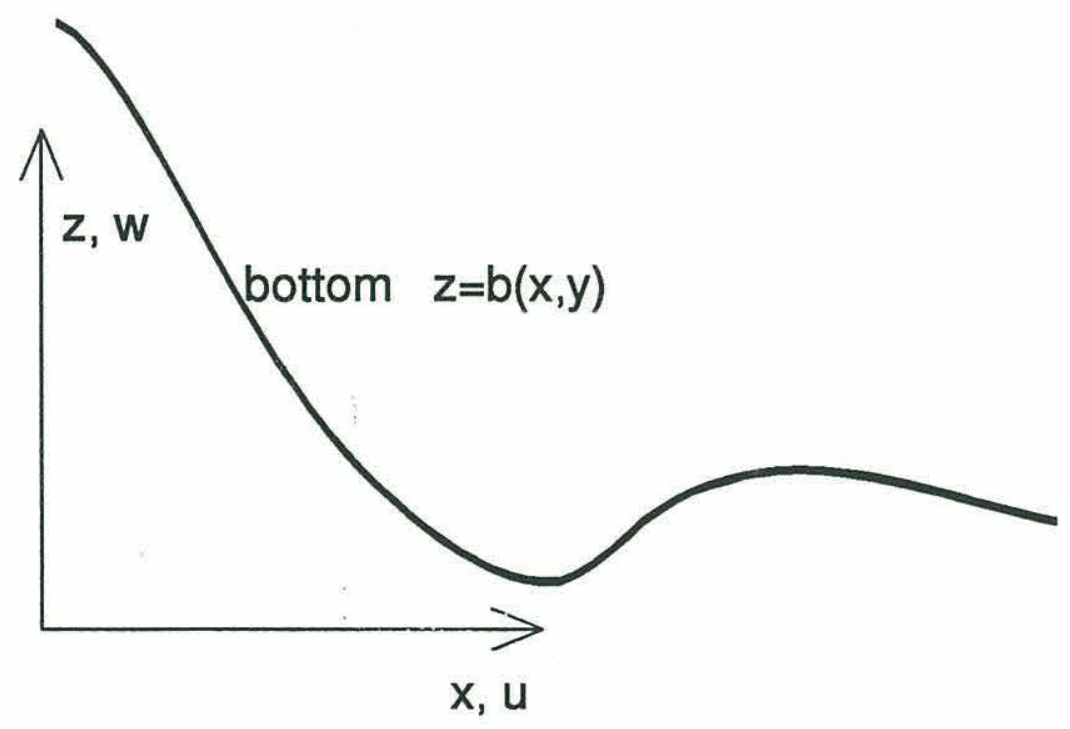

Figure 4.1 Stratified ocean with bottom topography 
Substituting (4.2) and (4.4) into the density conservation equation (4.1) we shall obtain:

$$
\rho_{t}^{\prime}+u\left(\rho_{x}^{\prime}+\rho_{z}^{\prime} b_{x}\right)+v\left(\rho_{y}^{\prime}+\rho_{z}^{\prime} b_{y}\right)+\rho_{0 z}\left(u b_{x}+v b_{y}\right)=0
$$

Expressions with $\rho^{\prime}$ in brackets have the meaning of horizontal ( $\mathrm{x}$ and $\mathrm{y}$ ) differentiation along the bottom - the surface $\mathrm{z}=\mathrm{b}(\mathrm{x}, \mathrm{y})$ :

$$
\rho_{x}^{\prime}+\rho_{z}^{\prime} b_{x}=\rho_{x}^{\prime} I_{\text {bot }}, \quad \rho_{y}^{\prime}+\rho_{z}^{\prime} b_{y}=\rho_{y}^{\prime} I_{b o t}
$$

This difference between the simple horizontal derivative and the derivative along the bottom (4.5) is of the order of a Rossby number and can be neglected in the QG approximation. However we shall keep distinguishing them for a while because all the above derivations are valid not only for QG but for any adiabatic dynamics.

Using $\rho_{0 \mathrm{z}}=-\rho_{0} \frac{\mathrm{N}^{2}}{\mathrm{~g}}$ we can rewrite (4.1') in the form

$$
\rho_{t}^{\prime}+u\left(\rho_{x}^{\prime} I_{b o t}-\rho_{0} \frac{N^{2}}{g} b_{x}\right)+v\left(\rho_{y}^{\prime} l_{b o t}-\rho_{0} \frac{N^{2}}{g} b_{y}\right)=0 \quad @ z=b(x, y)
$$

which implies a lagrangian density conservation when moving a water parcel along the bottom:

$$
\frac{d}{d t}\left(\rho^{\prime}+\int_{b}^{\infty} \rho_{0} \frac{N^{2}}{g} d z\right)=0
$$

Here and later in this section lagrangian time derivative $d / d t$ is two-dimensional along the bottom $\mathrm{z}=\mathrm{b}(\mathrm{x}, \mathrm{y})$ :

$$
\frac{d}{d t}=\partial_{t}+\left.u \partial_{x}\right|_{\text {bot }}+\left.v \partial_{y}\right|_{\text {bot }}
$$

Buoyancy frequency $\mathrm{N}$ can generally depend on $\mathrm{z}$ but in the equation (4.1") it is evaluated at the bottom, $\mathrm{z}=\mathrm{b}(\mathrm{x}, \mathrm{y})$ so that in this equation it is a function of horizontal coordinates $\mathrm{N}(\mathrm{x}, \mathrm{y})=\mathrm{N}(\mathrm{z}(\mathrm{x}, \mathrm{y}))$.

For the case $\mathrm{N}=$ const which we shall actually use throughout most of the following derivations (4.6) is reduced to a more simple relation (neglecting the constant $\rho_{0} \frac{\mathrm{N}(\mathrm{H})^{2}}{\mathrm{~g}} \mathrm{H}$, which is differentiated out in (4.6) ): 


$$
\frac{d}{d t}\left(\rho^{\prime}-\rho_{0} \frac{N^{2}}{g} b\right)=0
$$

Now we shall consider the quasigeostrophic approximation on the f-plane. We can introduce a streamfunction $\psi$, derivatives of which are related to horizontal velocities and a density perturbation:

$$
\mathrm{u}=-\psi_{\mathrm{y}}, \quad \mathrm{v}=\psi_{\mathrm{x}}, \quad \rho^{\prime}=-\rho_{0} \frac{\mathrm{f}}{\mathrm{g}} \psi_{\mathrm{z}}
$$

Substituting (4.7) into (4.6) we obtain the lagrangian conservation property for the function $\eta$ (proportional to density) which, using the streamfunction, can be written as follows:

$$
\eta_{\mathrm{t}}+\mathrm{J}(\psi, \eta)=0, \quad \eta=\psi_{\mathrm{z}}+\frac{\mathrm{N}^{2}}{\mathrm{f}} \mathrm{b}
$$

This function $\eta$ is expressed through the vertical derivative $\psi_{z}$ at the bottom so that using the evolutionary boundary condition (4.6") we can determine $\psi_{\mathrm{z}}$ at any given time and use it in the inversion procedure to obtain a velocity field. This fact will be of primary importance for the method proposed.

\subsection{Formulation of the problem}

Now we suppose that the buoyancy frequency is uniform, $\mathrm{N}=\mathrm{const}$. The quasigeostrophic potential vorticity (PV) will be a lagrangian conserved quantity and can be written in this case as a three-dimensional Laplacian of the streamfunction $\quad \mathrm{q}=\nabla_{3}^{2} \psi \quad$ using a "stretched" vertical coordinate $\tilde{\mathrm{z}}=\mathrm{zN} / \mathrm{f}$. Combining this interior equation and bottom and surface (rigid lid) boundary conditions the problem of determining the evolution of such flow can be poised as following:

$$
\begin{array}{lll}
\mathrm{q}_{\mathrm{t}}+\mathrm{J}(\psi, \mathrm{q})=0, & \widetilde{\mathrm{H}}>\widetilde{\mathrm{z}}>\widetilde{\mathrm{b}}(\mathrm{x}, \mathrm{y}) \\
\Psi_{\tilde{\mathrm{z}}}=0 & \text { @ } \tilde{\mathrm{z}}=\widetilde{\mathrm{H}} & \text { (surface) } \\
\widetilde{\eta}_{\mathrm{t}}+\mathrm{J}(\psi, \widetilde{\eta})=0 & \text { @ } \tilde{\mathrm{z}}=\widetilde{\mathrm{b}}(\mathrm{x}, \mathrm{y}) & \text { (bottom) }
\end{array}
$$


where $\quad \mathrm{q}=\nabla_{3}^{2} \psi, \quad \nabla_{3}^{2}=\partial_{\mathrm{xx}}^{2}+\partial_{\mathrm{yy}}^{2}+\partial_{z \mathrm{z}}^{2}, \quad \tilde{\eta}=\psi_{\tilde{z}}+\mathrm{Nb}(\mathrm{x}, \mathrm{y})$.

Here tilded are the corresponding values in the stretched vertical coordinate. Thus we have the evolution (lagrangian conservation) equations for two quantities: a potential vorticity in the interior and function $\widetilde{\eta}$ (density) in the bottom boundary condition.

As we already mentioned in the introduction to this chapter it is convenient to split the problem in two steps at each moment of time. First - knowing the distribution of $q(x, y, z)$ and $\widetilde{\eta}(x, y)$ and hence $\psi_{\tilde{\mathrm{z}}}(\mathrm{x}, \mathrm{y})$ at the bottom at a given time we can compute a streamfunction $\psi$ everywhere in the domain. Second, substitute $\psi$ into the evolution equations (4.6a), (4.6c) and obtain $q$ and $\widetilde{\eta}$ at the next time moment.

Consider now the first step in some more details. We must solve the following problem (the interior equation is always in the same domain $\widetilde{\mathrm{H}}>\widetilde{\mathrm{z}}>\widetilde{\mathrm{b}}(\mathrm{x}, \mathrm{y})$ unless indicated otherwise):

$$
\begin{array}{ll}
\nabla_{3}^{2} \psi=\mathrm{q}(\mathrm{x}, \mathrm{y}, \tilde{\mathrm{z}}) & \\
\psi_{\tilde{\mathrm{z}}}=0 & @ \quad \tilde{\mathrm{z}}=\widetilde{\mathrm{H}} \\
\psi_{\tilde{\mathrm{z}}}=\tilde{\eta}-\mathrm{N} \tilde{\mathrm{b}} & @ \quad \tilde{\mathrm{z}}=\tilde{\mathrm{b}}(\mathrm{x}, \mathrm{y})
\end{array}
$$

Computationally it is more convenient to divide the streamfunction into two parts:

$$
\psi=\psi^{\text {int }}+\psi^{\text {bot }}
$$

the (int) - for "interior" and (bot) - for "bottom". Here $\psi^{\text {int }}$ is the streamfunction induced by PV sources in the interior of an ocean without the presence of the bottom (in this case the domain is semi-infinite $\widetilde{\mathrm{H}}>\widetilde{\mathrm{z}}>-\infty)$. So that for $\psi^{\text {int }}$ we have the following problem:

$$
\begin{array}{ll}
\nabla_{3}^{2} \psi^{\text {int }}=q(\mathrm{x}, \mathrm{y}, \tilde{\mathrm{z}}) & \\
\psi_{\mathrm{z}}^{\text {int }}=0 & @ \quad \tilde{\mathrm{z}}=\widetilde{\mathrm{H}} \\
\psi^{\text {int }}=0 & @ \quad \tilde{\mathrm{z}} \rightarrow-\infty
\end{array}
$$

The other part, $\psi^{\text {bot }}$, is subject to the Laplace equation in the interior and, added to $\psi^{\text {int }}$ must satisfy the bottom boundary condition $(4.7 \mathrm{c})$ :

$$
\nabla_{3}^{2} \psi^{\text {bot }}=0
$$




$$
\begin{array}{ll}
\psi_{\tilde{\mathrm{z}}}^{\mathrm{bot}}=0 & @ \quad \tilde{\mathrm{z}}=\widetilde{\mathrm{H}} \\
\psi_{\tilde{\mathrm{z}}}^{\mathrm{bot}}=\widetilde{\eta}-\mathrm{N} \tilde{\mathrm{b}}-\psi_{\tilde{\mathrm{z}}}^{\mathrm{int}} & @ \quad \tilde{\mathrm{z}}=\tilde{\mathrm{b}}(\mathrm{x}, \mathrm{y})
\end{array}
$$

The vertical derivative $\Psi_{\tilde{\mathrm{z}}}^{\text {bot }}$ in $(4.10 \mathrm{c})$ is related to the position of isopycnals intersection (that is the function $\widetilde{\eta}(\mathrm{x}, \mathrm{y})$ ) and includes also a "leftover" $\psi_{\tilde{\mathrm{z}}}^{\text {int }}$ from the "interior" part.

\subsection{Green's function for PV and density anomalies for the flat bottom case}

Both (4.9) and (4.10) are linear problems we can express the solutions $\psi^{\text {bot }}$ and $\psi^{\text {int }}$ in terms of the corresponding Green's functions. Thus for $\psi^{\text {int }}$ we have:

$$
\begin{aligned}
& \psi^{\text {int }}(\mathbf{R})=\int q\left(\mathbf{R}^{\prime}\right) G^{\text {int }}\left(\mathbf{R} \mid \mathbf{R}^{\prime}\right) d^{3} \mathbf{R}^{\prime} \\
& G^{\text {int }}\left(\mathbf{R} \mid \mathbf{R}^{\prime}\right)=-\frac{1}{4 \pi}\left(\frac{1}{\sqrt{\left(x-x^{\prime}\right)^{2}+\left(y-y^{\prime}\right)^{2}+\left(\tilde{z}-\tilde{z}^{\prime}\right)^{2}}}+\frac{1}{\sqrt{\left(x-x^{\prime}\right)^{2}+\left(y-y^{\prime}\right)^{2}+\left(\tilde{z}-2 \widetilde{H}+\widetilde{z}^{\prime}\right)^{2}}}\right)
\end{aligned}
$$

where $\mathbf{R}=(\mathrm{x}, \mathrm{y}, \tilde{\mathrm{z}}), \quad \mathbf{R}^{\prime}=\left(\mathrm{x}^{\prime}, \mathrm{y}^{\prime}, \tilde{\mathrm{z}}^{\prime}\right)$

This means that the Green's function $G^{\text {int }}\left(\mathbf{R} \mid \mathbf{R}^{\prime}\right)=G^{\text {int }}\left(\mathbf{r}-\mathbf{r}^{\prime}, \tilde{z} \mid \tilde{z}^{\prime}\right)$ is identical to a field of a point charge plus its like-signed image symmetrically placed relative to the plane $\tilde{\mathrm{Z}}=\widetilde{\mathrm{H}}$.

The problem (4.10) for $\psi^{\text {bot }}$ is generally more difficult. Again we can express the solution in terms of convolution with a Green's function but the integration is done now over the twodimensional variable $\mathbf{r}^{\prime}$. As we already did before by the small letter $\mathbf{r}$ we shall denote twodimensional vectors $\mathbf{r}=(\mathrm{x}, \mathrm{y})$ and by the capital $\mathbf{R}$ or the combination $(\mathbf{r}, \tilde{\mathrm{z}})$ - three-dimensional vectors, with corresponding upper or lower indices:

$$
\psi^{\text {bot }}(\mathbf{R})=\int \phi\left(\mathbf{r}^{\prime}\right) G^{\text {bot }}\left(\mathbf{R} \mid \mathbf{r}^{\prime}\right) d^{2} \mathbf{r}^{\prime}
$$


where $\quad \mathbf{r}^{\prime}=\left(x^{\prime}, y^{\prime}\right), \quad \phi=-N(\tilde{\eta}+\tilde{b})-\psi_{\tilde{z}}^{\text {int }}$

Now $G^{\text {bot }}$ is a solution of a following problem:

$$
\begin{aligned}
& \nabla_{3}^{2} \mathrm{G}^{\mathrm{bot}}=0 \\
& \mathrm{G}_{\tilde{\mathrm{z}}}^{\text {bot }}=0 \quad \text { @ } \quad \tilde{\mathrm{z}}=\widetilde{\mathrm{H}} \\
& \mathrm{G}_{\widetilde{\mathrm{z}}}^{\text {bot }}=\delta\left(\mathrm{x}-\mathrm{x}^{\prime}, \mathrm{y}-\mathrm{y}^{\prime}\right) \quad @ \quad \tilde{\mathrm{z}}=\widetilde{\mathrm{b}}(\mathrm{x}, \mathrm{y})
\end{aligned}
$$

We consider at first the simplest case when the bottom is flat $\tilde{\mathrm{b}}(\mathrm{x}, \mathrm{y})=\widetilde{\mathrm{b}}_{0}$. Then the Green's function (4.13) is again easily found:

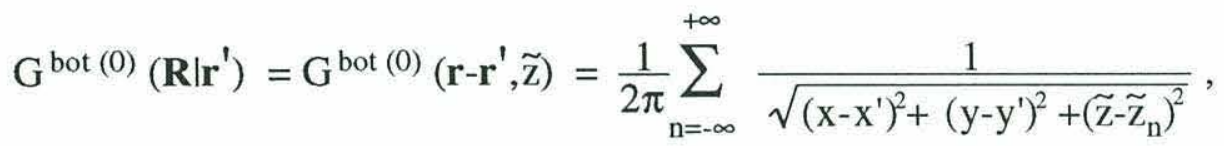

where $\quad \tilde{\mathrm{z}}_{\mathrm{n}}=\widetilde{\mathrm{b}}_{0}-0+2 \mathrm{n}\left[\tilde{\mathrm{H}}-\left(\widetilde{\mathrm{b}}_{0}-0\right)\right]$

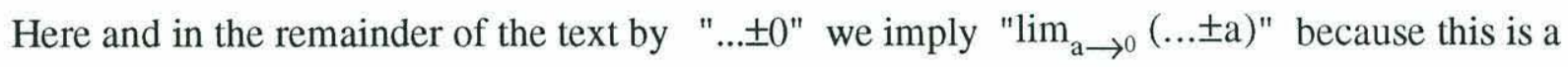
singular limit and the solution will not be correct if we omit " $\pm 0 "$.

Without the surface only one term in (4.14) survives (with $\mathrm{n}=0$ ) and it means that a "charge" must be put just under the bottom at the point $\left(x^{\prime}, y^{\prime}, \widetilde{b}_{0}-0\right)$; the vertical derivative of its field will exactly generate the delta-function for $(4.13 \mathrm{c})$ :

$$
\frac{+0}{{\sqrt{0^{2}+\left(\mathbf{r}-\mathbf{r}_{0}\right)^{2}}}^{3}}=2 \pi \delta\left(\mathbf{r}-\mathbf{r}_{0}\right)
$$


"Image" solution for Green's function

. $\mathrm{z}=2 \mathrm{H}+\mathrm{h}$

- - - - - - - - - - - - - - - - -

surface, $z=H \quad \mathrm{z}=2 \mathrm{H}-\mathrm{h}$

PV anomaly, $z=h$

bottom, $z=0$

image, $z=-h$

- - - - - - - - - - - - - - - - -

$\mathrm{z}=-2 \mathrm{H}+\mathrm{h}$

Figure 4.2 System of images for the flat bottom case 
When the surface is present a system of images must be added as shown in figure 4.2 and which is represented by terms with $\mathrm{n} \neq 0$ in the sum (4.14).

\subsection{Green's function for a variable bottom}

For the QG approximation we can use the Green's function for the problem with a flat bottom. But the proposed method can be extended to more general equations of motion and in that case the corrections to the Green's function are needed. When the bottom is not flat the Green's function $\mathrm{G}^{\text {bot }}$ will generally depend on both arguments, $\mathbf{R}$ and $\mathbf{r}^{\prime}$ and not only on the difference $\mathbf{r}-\mathbf{r}^{\prime}$ as in the case of $G^{\text {int }}$ and $G^{\text {bot }(0)}$ which makes the problem more complicated. However if the bottom is smooth and its typical slope angle is small then $G^{\text {bot }}$ will have a similar structure as

$\mathrm{G}^{\text {bot }(0)}$ but slightly modified. For the sake of simplicity we consider at first a semi-infinite ocean without the surface. We shall seek the Green's function as a potential induced by a "distributed charge" with a density $\sigma\left(\mathbf{r}, \mathbf{r}_{0}\right)$ again put just under the bottom:

$$
\mathrm{G}^{(\mathrm{b})}\left(\mathbf{R} \mid \mathbf{r}_{0}\right)=-\iint \frac{\sigma\left(\mathbf{r}^{\prime}, \mathbf{r}_{0}\right)}{\sqrt{\left(\mathbf{r}^{\prime}-\mathbf{r}^{\prime}\right)^{2}+\left[\tilde{z}-\tilde{b}\left(\mathbf{r}^{\prime}\right)+0\right]^{2}}} d x^{\prime} d y^{\prime}
$$

(We shall need at least three two-dimensional variables $-\mathbf{r}, \mathbf{r}_{0}, \mathbf{r}^{\prime} ; \quad \mathbf{r}$ and $\mathbf{r}_{0}$ will be used in Green's function $\mathrm{G}_{\tilde{z}}^{\text {bot }}$ so that $\mathrm{G}_{\tilde{\mathbf{z}}}^{\text {bot }}\left(\mathbf{r} \mid \mathbf{r}_{0}\right)=\delta\left(\mathbf{r}-\mathbf{r}_{0}\right)$ at the bottom and $\mathbf{r}^{\prime}$ - variable of integration in convolution of charge density $\sigma\left(\mathbf{r}^{\prime}, \mathbf{r}_{0}\right)$ with the field from the point charge as expressed in (4.16) ).

Substituting (4.16) into bottom boundary condition (4.13c) we obtain:

$$
\mathrm{G}_{\mathrm{z}}^{\text {bot }}\left(\mathbf{r} \mid \mathbf{r}_{0}\right)=\iint \sigma\left(\mathbf{r}^{\prime}, \mathbf{r}_{0}\right) \frac{\left[\tilde{\mathrm{b}}(\mathbf{r})-\tilde{\mathrm{b}}\left(\mathbf{r}^{\prime}\right)+0\right]}{\sqrt{\left(\mathbf{r}-\mathbf{r}^{\prime}\right)^{2}+\left[\tilde{\mathrm{b}}(\mathbf{r})-\widetilde{\mathrm{b}}\left(\mathbf{r}^{\prime}\right)+0\right]^{2}}} d x^{\prime} d y^{\prime}=\delta\left(\mathbf{r}-\mathbf{r}_{0}\right)
$$

Now we shall use the fact that $|\nabla \widetilde{\mathrm{b}}|<<1$ and introduce a small parameter $\varepsilon$ so that $|\nabla \widetilde{\mathrm{b}}|=\mathrm{O}(\varepsilon)$. We can make it, for example, by expressing the topography at each point $\mathbf{r}_{0}$ as a polynomial function in such a way: 


$$
\widetilde{\mathrm{b}}(\mathbf{r})=\widetilde{\mathrm{b}}\left(\mathbf{r}_{0}\right)+\varepsilon \mathrm{B}\left(\mathbf{r}, \mathbf{r}_{0}\right), \quad \mathrm{B}\left(\mathbf{r}, \mathbf{r}_{0}\right)=\sum_{\mathrm{p}=0}^{\mathrm{m}} \sum_{\mathrm{q}=0, \mathrm{pq} \neq 0}^{\mathrm{n}} \mathrm{b}_{\mathrm{pq}}\left(\mathrm{x}-\mathrm{x}_{0}\right)^{\mathrm{p}}\left(\mathrm{y}-\mathrm{y}_{0}\right)^{\mathrm{q}}
$$

where the coefficients $b_{p q}\left(\mathbf{r}, \mathbf{r}_{0}\right)$ are of the order $\mathrm{O}(1)$.

We also expand the "charge density" $\sigma\left(\mathbf{r}, \mathbf{r}_{0}\right)$ in powers of parameter $\varepsilon$ :

$$
\sigma\left(\mathbf{r}, \mathbf{r}_{0}\right)=\sigma^{(0)}\left(\mathbf{r}, \mathbf{r}_{0}\right)+\varepsilon \sigma^{(1)}\left(\mathbf{r}, \mathbf{r}_{0}\right)+\varepsilon^{2} \sigma^{(2)}\left(\mathbf{r}, \mathbf{r}_{0}\right)+O\left(\varepsilon^{3}\right)
$$

Substituting (4.18) and (4.19) into the expression (4.17) up to the terms of the order $\mathrm{O}\left(\varepsilon^{2}\right)$ we can write:

$$
\begin{aligned}
& \mathrm{G}_{\tilde{\mathbf{z}}}^{\text {bot }}\left(\mathbf{r}, \mathbf{r}_{0}\right)=\iint\left[\sigma^{(0)}\left(\mathbf{r}^{\prime}, \mathbf{r}_{0}\right)+\varepsilon \sigma^{(1)}\left(\mathbf{r}^{\prime}, \mathbf{r}_{0}\right)+\varepsilon^{2} \sigma^{(2)}\left(\mathbf{r}^{\prime}, \mathbf{r}_{0}\right)\right] \frac{\varepsilon\left[\mathrm{B}\left(\mathbf{r}, \mathbf{r}_{0}\right)-\mathrm{B}\left(\mathbf{r}^{\prime}, \mathbf{r}_{0}\right)\right]+0}{\sqrt{\left(\mathbf{r}-\mathbf{r}^{\prime}\right)^{2}+\left\{\varepsilon\left[\mathrm{B}\left(\mathbf{r}, \mathbf{r}_{0}\right)-\mathrm{B}\left(\mathbf{r}^{\prime}, \mathbf{r}_{0}\right)\right]+0\right\}^{2}}} d^{2} \mathbf{r}^{\prime}= \\
& =\iint \sigma^{(0)}\left(\mathbf{r}^{\prime}, \mathbf{r}_{0}\right) \frac{(+0)}{{\sqrt{\left(\mathbf{r}-\mathbf{r}^{\prime}\right)^{2}+0^{2}}}^{3}} d^{2} \mathbf{r}^{\prime}+\varepsilon \iint\left\{\sigma^{(1)}\left(\mathbf{r}^{\prime}, \mathbf{r}_{0}\right) \frac{+0}{\sqrt{\ldots}^{3}}+\sigma^{(0)}\left(\mathbf{r}^{\prime}, \mathbf{r}_{0}\right) \frac{\mathrm{B}\left(\mathbf{r}, \mathbf{r}_{0}\right)-\mathrm{B}\left(\mathbf{r}^{\prime}, \mathbf{r}_{0}\right)}{\sqrt{\ldots}^{3}}\right\} \mathrm{d}^{2} \mathbf{r}^{\prime}+ \\
& +\varepsilon^{2} \iint\left\{\sigma^{(2)}\left(\mathbf{r}^{\prime}, \mathbf{r}_{0}\right) \frac{+0}{\sqrt{\ldots}^{3}}+\sigma^{(1)}\left(\mathbf{r}^{\prime}, \mathbf{r}_{0}\right) \frac{\left[\mathrm{B}\left(\mathbf{r}, \mathbf{r}_{0}\right)-\mathrm{B}\left(\mathbf{r}^{\prime}, \mathbf{r}_{0}\right)\right]}{\sqrt{\ldots}^{3}}-\frac{3}{2} \sigma^{(0)}\left(\mathbf{r}^{\prime}, \mathbf{r}_{0}\right) \frac{\left[\mathrm{B}\left(\mathbf{r}, \mathbf{r}_{0}\right)-\mathrm{B}\left(\mathbf{r}^{\prime}, \mathbf{r}_{0}\right)\right]}{\sqrt{\ldots}^{5}}\right\}^{2} \mathrm{~d}^{2} \mathbf{r}^{\prime}= \\
& =\delta\left(\mathbf{r}-\mathbf{r}_{0}\right)
\end{aligned}
$$

where in all the square roots in the denominators there is an expression $\left(\mathbf{r}-\mathbf{r}^{\prime}\right)^{2}+0^{2}$. Equating the terms at each power of $\varepsilon$ and using the relation (4.15) we immediately obtain:

$$
\begin{aligned}
& \sigma^{(0)}\left(\mathbf{r}, \mathbf{r}_{0}\right)=\frac{1}{2 \pi} \delta\left(\mathbf{r}-\mathbf{r}_{0}\right) \\
& \sigma^{(1)}\left(\mathbf{r}, \mathbf{r}_{0}\right)=-\frac{1}{4 \pi^{2}} \frac{\mathrm{B}\left(\mathbf{r}, \mathbf{r}_{0}\right)}{{\sqrt{\left(\mathbf{r}-\mathbf{r}_{0}\right)^{2}+0^{2}}}^{3}}
\end{aligned}
$$




$$
\sigma^{(2)}\left(\mathbf{r}, \mathbf{r}_{0}\right)=\frac{1}{8 \pi^{3}} \iint \frac{\mathrm{B}\left(\mathbf{r}^{\prime}, \mathbf{r}_{0}\right)}{{\sqrt{\left(\mathbf{r}^{\prime}-\mathbf{r}_{0}\right)^{2}+0^{2}}}^{3}} \frac{\mathrm{B}\left(\mathbf{r}, \mathbf{r}_{0}\right)-\mathrm{B}\left(\mathbf{r}^{\prime}, \mathbf{r}_{0}\right)}{{\sqrt{\left(\mathbf{r}^{\prime}-\mathbf{r}\right)^{2}+0^{2}}}^{3}} \mathrm{~d}^{2} \mathbf{r}^{\prime}-\frac{3}{2} \frac{\mathrm{B}\left(\mathbf{r}, \mathbf{r}_{0}\right)^{2}}{{\sqrt{\left(\mathbf{r}-\mathbf{r}_{0}\right)^{2}+0^{2}}}^{5}}
$$

In the zero-order approximation we obtain the same result as for a flat bottom (without the surface). This coincides with a widely used approximation when the bottom boundary condition is related to "nominal" depth $\mathrm{z}=\mathrm{b}_{0}$. The first approximation means that we put at each point under the bottom a "distributed charge" of such density that the vertical derivative from its field will exactly cancel that of a "point charge" from the zero-order approximation. For the second approximation we should make the same procedure as for the first plus there will be another term due to the expansion of the denominator in powers of $\varepsilon$. Because $\varepsilon$ must be a small parameter we expect that for most cases first or even zero approximation will be sufficient.

When the surface is present we can perform a similar expansion as the one described above. Again we can sum up the "distributed charge" under the bottom and all its "images" above and below:

$$
\mathrm{G}^{\text {bot }}\left(\mathbf{R} \mid \mathbf{r}_{0}\right)=-\sum_{\mathrm{n}=-\infty}^{+\infty} \iint \frac{\sigma_{\mathrm{n}}\left(\mathbf{r}^{\prime}, \mathbf{r}_{0}\right)}{\sqrt{\left(\mathbf{r}^{\prime}-\mathbf{r}_{0}\right)^{2}+\left(\widetilde{\mathrm{z}}-\widetilde{\mathbf{z}}_{\mathrm{n}}\right)^{2}}} d^{2} \mathbf{r}^{\prime}
$$

where $\quad \tilde{\mathrm{z}}_{\mathrm{n}}{ }^{\prime}=\widetilde{\mathrm{b}}\left(\mathbf{r}^{\prime}\right)-0+2 \mathrm{n}\left[\widetilde{\mathrm{H}}-\widetilde{\mathrm{b}}\left(\mathbf{r}^{\prime}\right)+0\right]$.

At the bottom $\tilde{\mathrm{z}}=\tilde{\mathrm{b}}(\mathbf{r})$ so that

$$
\begin{gathered}
\left(\widetilde{z}-\tilde{\mathbf{z}}_{\mathbf{n}}\right)^{2}=0^{2}(1-2 n)^{2}+4 n^{2}\left[\widetilde{\mathrm{H}}-\widetilde{\mathrm{b}}\left(\mathbf{r}_{0}\right)\right]^{2}-\varepsilon 4 \mathrm{n}\left[\mathrm{B}\left(\mathbf{r}, \mathbf{r}_{0}\right)-(2 n+1) B\left(\mathbf{r}^{\prime}, \mathbf{r}_{0}\right)\right]\left[\widetilde{\mathrm{H}}-\widetilde{\mathrm{b}}\left(\mathbf{r}_{0}\right)\right]+ \\
+\varepsilon^{2} 4 n^{2}\left[\mathrm{~B}\left(\mathbf{r}, \mathbf{r}_{0}\right)-(2 n+1) B\left(\mathbf{r}^{\prime}, \mathbf{r}_{0}\right)\right]^{2}
\end{gathered}
$$

And

$$
\mathrm{G}_{\tilde{\mathrm{z}}}^{\mathrm{bot}}\left(\mathbf{r} \mid \mathbf{r}_{0}\right)=\sum_{\mathrm{n}=-\infty}^{+\infty} \iint \sigma_{\mathrm{n}}\left(\mathbf{r}^{\prime}, \mathbf{r}_{0}\right) \frac{\widetilde{\mathrm{b}}(\mathbf{r})-\tilde{z}_{\mathrm{n}}{ }^{\prime}}{\sqrt{\left(\mathbf{r}^{\prime}-\mathbf{r}_{0}\right)^{2}+\left[\widetilde{\mathrm{b}}(\mathbf{r})-\tilde{\mathrm{z}}_{\mathrm{n}}\right]^{2}}{ }^{3}} d^{2} \mathbf{r}^{\prime}
$$

For the square root in the denominator in the convolution integral we obtain following expansion in the parameter $\varepsilon$ : 


$$
\begin{aligned}
\frac{1}{\sqrt{\left(\mathbf{r}-\mathbf{r}^{\prime}\right)^{2}+\left[\widetilde{\mathrm{b}}(\mathbf{r})-\widetilde{\mathrm{Z}}_{\mathrm{n}}^{\prime}\right]^{2}}} & =\frac{1}{\sqrt{0^{2}(1-2 \mathrm{n})^{2}+\left(\mathbf{r}-\mathbf{r}^{\prime}\right)^{2}+4 \mathrm{n}^{2}\left[\widetilde{\mathrm{H}}-\widetilde{\mathrm{b}}\left(\mathbf{r}_{0}\right)\right]^{23}} \times} \\
& \left(1+\varepsilon \frac{4 \mathrm{n}\left[\mathrm{B}\left(\mathbf{r}, \mathbf{r}_{0}\right)-(2 \mathrm{n}+1) \mathrm{B}\left(\mathbf{r}^{\prime}, \mathbf{r}_{0}\right)\right]\left[\widetilde{\mathrm{H}}-\widetilde{b}\left(\mathbf{r}_{0}\right)\right]}{\left(\mathbf{r}-\mathbf{r}^{\prime}\right)^{2}+4 \mathrm{n}^{2}\left[\widetilde{\mathrm{H}}-\tilde{b}\left(\mathbf{r}_{0}\right)\right]^{2}}\right)+O\left(\varepsilon^{2}\right)
\end{aligned}
$$

Again substituting the expansion of a density charge (4.19) into (4.23) we obtain a relation analogous to (4.20) for a semi-infinite ocean:

$$
\begin{aligned}
& \mathrm{G}_{\tilde{\mathrm{z}}}^{\mathrm{bot}}\left(\mathbf{r} \mid \mathbf{r}_{0}\right)=\sum_{\mathrm{n}=-\infty}^{+\infty} \iint \sigma_{\mathrm{n}}^{(0)}\left(\mathbf{r}^{\prime}, \mathbf{r}_{0}\right) \frac{+0}{\sqrt{0^{2}+\left(\mathbf{r}^{\prime}-\mathbf{r}_{0}\right)^{2}+4 \mathrm{n}^{2}\left[\widetilde{\mathrm{H}}-\widetilde{\mathrm{b}}\left(\mathbf{r}_{0}\right)\right]^{2}}} d^{2} \mathbf{r}^{\prime} \\
& +\varepsilon \sum_{\mathrm{n}=-\infty}^{+\infty} \iint\left(\sigma_{\mathrm{n}}^{(1)}\left(\mathbf{r}^{\prime}, \mathbf{r}_{0}\right) \frac{+0}{\sqrt{\ldots}^{3}}+\sigma_{\mathrm{n}}^{(0)}\left(\mathbf{r}^{\prime}, \mathbf{r}_{0}\right) \frac{\mathrm{B}\left(\mathbf{r}, \mathbf{r}_{0}\right)-\mathrm{B}\left(\mathbf{r}^{\prime}, \mathbf{r}_{0}\right)}{\sqrt{\ldots}^{3}}\right) d^{2} \mathbf{r}^{\prime}+ \\
& +\varepsilon \sum_{\mathrm{n}=-\infty}^{+\infty} \iint \sigma_{\mathrm{n}}^{(0)}\left(\mathbf{r}^{\prime}, \mathbf{r}_{0}\right) 6 \mathrm{n} \frac{\left[\mathrm{B}\left(\mathbf{r}, \mathbf{r}_{0}\right)-(2 \mathrm{n}+1) \mathrm{B}\left(\mathbf{r}^{\prime}, \mathbf{r}_{0}\right)\right]\left[\widetilde{\mathrm{H}}-\widetilde{\mathrm{b}}\left(\mathbf{r}_{0}\right)\right]}{\sqrt{\ldots}^{5}} d^{2} \mathbf{r}^{\prime}+\mathrm{O}\left(\varepsilon^{2}\right)
\end{aligned}
$$

And for the "charge density" $\sigma\left(\mathbf{r}, \mathbf{r}_{0}\right)$ we have (for simplicity we shall write only first two approximations; procedure for deriving the next approximations is relatively straightforward):

$$
\begin{gathered}
\sigma_{\mathrm{n}}^{(0)}\left(\mathbf{r}, \mathbf{r}_{0}\right)=\frac{1}{2 \pi} \delta\left(\mathbf{r}-\mathbf{r}_{0}\right) \\
\sigma_{\mathrm{n}}^{(1)}=-\frac{1}{4 \pi^{2}}\left(\frac{\mathrm{B}\left(\mathbf{r}, \mathbf{r}_{0}\right)}{\sqrt{0^{2}+\left(\mathbf{r}^{\prime}-\mathbf{r}_{0}\right)^{2}+4 \mathrm{n}^{2}\left[\widetilde{\mathrm{H}}-\widetilde{\mathrm{b}}\left(\mathbf{r}_{0}\right)\right]^{2}}}+6 \mathrm{n} \frac{\mathrm{B}\left(\mathbf{r}, \mathbf{r}_{0}\right)\left[\widetilde{\mathrm{H}}-\widetilde{\mathrm{b}}\left(\mathbf{r}_{0}\right)\right]}{\sqrt{\ldots}^{5}}\right)
\end{gathered}
$$

All terms except the last one in the second equation in (4.25) have corresponding terms (for $\mathrm{n}=0$ ) in (4.21) for a semi-infinite ocean.

And, finally, the Green's function at each approximation is obtained similarly to (4.16) as a sum of convolution integrals of $\sigma\left(\mathbf{r}, \mathbf{r}_{0}\right)$ and all images:

$$
\mathrm{G}^{\text {bot }}{ }^{(\mathrm{i})}\left(\mathbf{R} \mid \mathbf{r}_{0}\right)=-\sum_{\mathrm{n}=-\infty}^{+\infty} \iint \frac{\sigma_{\mathrm{n}}^{(\mathrm{i})}\left(\mathbf{r}^{\prime}, \mathbf{r}_{0}\right)}{\sqrt{0^{2}+\left(\mathbf{r}-\mathbf{r}^{\prime}\right)^{2}+4 n^{2}\left[\widetilde{\mathrm{H}}-\widetilde{\mathrm{b}}\left(\mathbf{r}_{0}\right)\right]^{2}}} d^{2} \mathbf{r}^{\prime}
$$


For many situations even the zero-order approximation is sufficient because it introduces an error of the order $\varepsilon$ (steepness of the topographic slope in the "stretched" coordinates). This should be comparable to the Rossby number which is the degree of accuracy of the quasigeostrophic approximation itself. And in the numerical algorithm which we shall describe below and which is based on the theoretical considerations of this chapter we shall use mainly this zero-order approximation, remembering though that if necessary the first approximation can be obtained using (4.26) with (4.25) or (4.16) with (4.21) for absence and presence of the surface respectively.

\subsection{Numerical implementation in a semi-lagrangian form}

As we already mentioned at the beginning of this chapter, conservation of potential vorticity and density for fluid parcels suggests usefulness of lagrangian numerical methods for the flow computation, for example of the contour dynamics (CD) type. Our actual numerical implementation is considerably different from the traditional $\mathrm{CD}$ form and contains some features pertinent to a purely Lagrangian (CD) and an Eulerian (fixed regular grid) approach. Below we will try briefly to explain the reasons why we chose this combined form.

In the standard $\mathrm{CD}$ algorithm a (potential) vorticity field is represented by a step-like distribution. This, if viewed as an approximation of a continuous field, gives generally a secondorder error in the velocity field. But for turbulent two-dimensional flows this can actually be a more realistic approximation than gridded or spectral models, since such flows are known to develop localized structures with high gradients. Therefore this step-like field can be a better description for some purposes than a projection on a regular grid or some spectral basis. But in the case of bottom density field for continuous stratification it is likely to be a smooth continuous function, and a steplike form is less accurate there. Another problem for the purely Lagrangian contour dynamics technique is that when the number of contours and moving points is large enough the inversion procedure via the convolution integral along the contours becomes very inefficient. We have to calculate the Green's function depending on distances between each pair of points of irregular moving contours. In this case (many irregular contours) we practically lose the initially perceived advantage of the contour dynamics method - reduction of a two-dimensional problem to a onedimensional integration.

In our model we use a mixed approach: while the advection problem is implemented by a lagrangian method (moving contours), the inversion procedure is performed on a regular grid, which 
is much more efficient than the convolution of a Green' function along the irregular contours. There is a price we have to pay for this combination - we need a connection between the Lagrangian and Eulerian description. This connection is provided by the interpolation procedure performed at each timestep from moving contours onto a regular grid and back to contours.

The main steps of our algorithm are as follows. Note that we shall mention density contours although the same operations can be applied also to vorticity contours if needed:

At the beginning of each step we suppose to know positions of density contours and values of density field on them. Then the routine is the following:

First - we interpolate a density field from moving contours onto a regular grid (see figure 4.3) using high-order Lagrange polynomials. We obtain a density field and estimates of its horizontal derivatives up to second order on the grid.

Second - we invert a density field for velocities on the same grid. Velocities induced by the density field are added to the velocities from interior potential vorticity anomalies (a single vortex in most of our experiments). Velocities are also computed at the points where PV anomalies themselves are situated.

Third - the velocity field at the bottom (on the grid) is interpolated back onto moving contours.

Fourth - the time-stepping procedure. Positions of the contours are advanced according to the advection velocity field. Since the time-stepping procedure is of the ordinary (not partial) differential equations type it allows rather big time steps. We use the accurate enough predictor - corrector scheme of the fourth order. 


\section{moving contours and underlying grid}

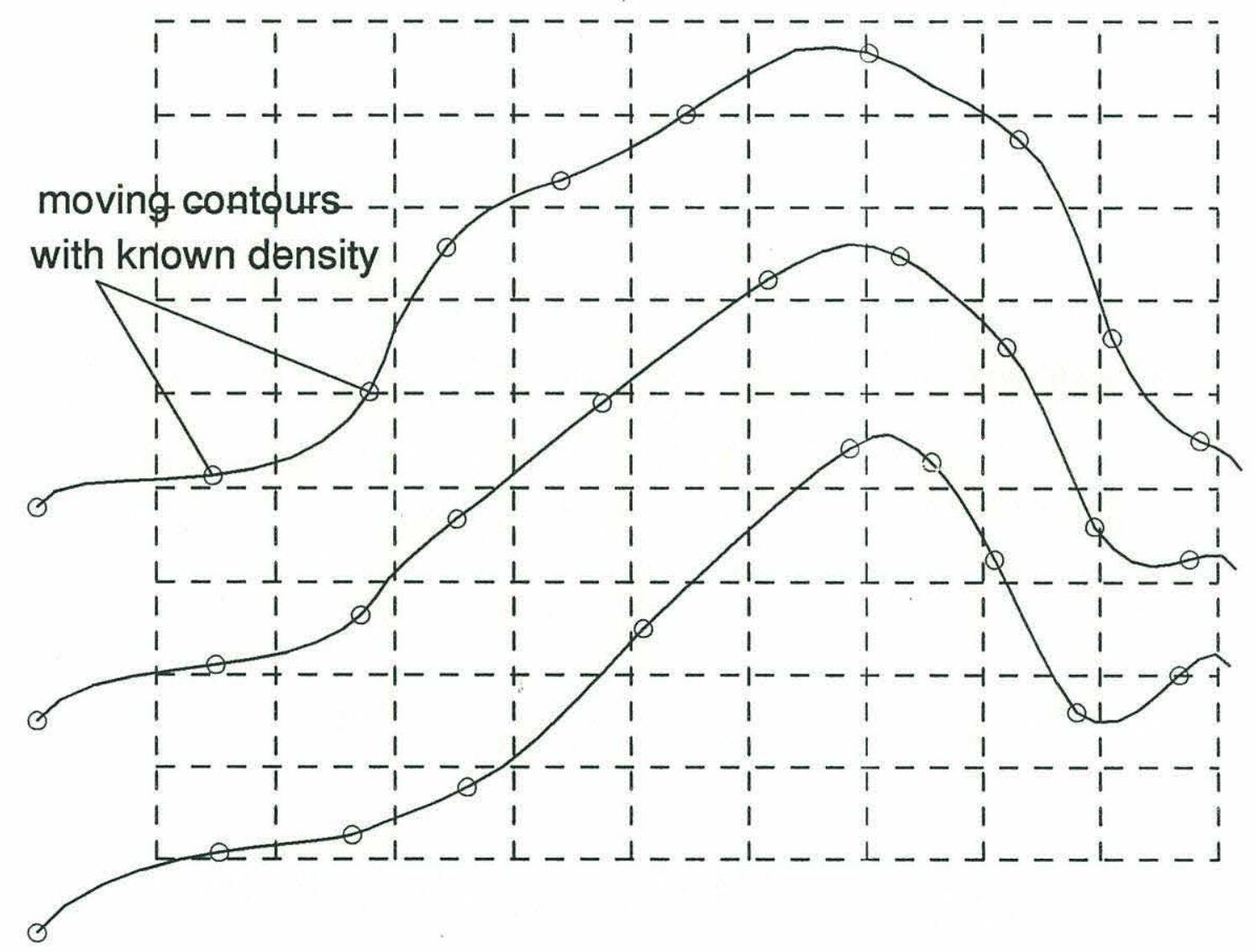

Figure 4.3 Lagrangian moving contours and an Eulerian regular grid 
There are also some additional numerical procedures needed to improve performance of the model. In particular we use slight smoothing of the contours in order to prevent their spurious intersections. We also employ a sponge-like relaxation of the density field at the radiative end of the domain. These numerical procedures and interpolation and inversion techniques are described in some more details in the Appendix 2.

To our knowledge this is the first attempt to combine a Lagrangian and Eulerian approach in a CD-type (as opposed to, say, finite-difference) method. And because of that we would like to reiterate the advantages which we see in this combination.

Among the advantages of the Lagrangian description of fluid flows there are:

- Automatic conservation of properties for fluid parcels.

We need only to advance the positions of points with known properties (PV, density) without computing spatial derivatives of their distributions. This is however satisfied exactly only for parcels on the contours themselves, because we interpolate between them with some loss of accuracy. Still if the interpolation is accurate enough it does not introduce a significant error.

- Time-stepping is in the ordinary differential equations (ODE) sense instead of PDE.

Instead of the awkward nonlinear advective term in the Euler equations we have a simple ODE time derivative: $\quad\left(\partial_{\mathrm{t}}+\mathrm{u} \partial_{\mathrm{x}}+\mathrm{v} \partial_{\mathrm{y}}\right)(*) \rightarrow(*)$.

- Inversion of the Poisson equation involves convolution with a Green's function, which is an integral and not a finite-difference procedure which has apparent advantage numerically. In particular it allows cruder resolution for the same accuracy and, conversely, better accuracy with the same resolution as finite-difference models. We shall have some more comments on the inversion with a Green's function in the Appendix 2.

- Numerical stability and robustness is firmly established for CD-type algorithms. This is probably due to a combination of the above mentioned properties. In particular, there is no Couranttype limitations for time steps, associated with the information propagation, since velocities at each point are influenced simultaneously by all other points of the domain. This is the advantage of a convolution with a long-range Green's function instead of use of a narrow-banded matrix - a finitedifference approximation of the Laplace operator.

- Resolution is adjusted where we need it. In the regions of large gradients we have many contours, while more quiet regions are less sampled. When the distance between contours becomes large to perform accurate enough interpolation procedure, the algorithm allows to insert new points or contours in the empty places.

The Eulerian approach is better suited for the following purposes: 
- It allows representation of continuous (and not only step-like) fields. This is particularly important for the density field at the bottom. While one can imagine localized patches of (potential) vorticity in a uniform background, a density anomaly field will likely be continuous and not localized in a small patch (this is at least the case in our calculations which we shall describe in the next chapters). Representing continuous fields by steps introduces a quadratic (with the number of steps) error in the velocity field, while treating it in a continuous function and interpolating on a regular grid increases the accuracy up to the fourth order in our model.

- A regular grid is easier to manipulate than irregular lagrangian contours. This leads to a more efficient inversion procedure than in the standard $\mathrm{CD}$ technique, where the inversion takes the most of computing time.

- The combined approach allows generalizations to more complicated dynamical models, for example to primitive equations. This is possible because dynamical variables do not have to be constant along the contours as in classical CD method but can change spatially and temporarily since we interpolate them on a regular grid at each time step. I do not discuss these generalizations here, but work is in progress to construct similar algorithm within semi-geostrophic and shallowwater dynamics.

- In the semi-lagrangian form it is easier (in comparison with the pure $\mathrm{CD}$ ) to incorporate forcing, dissipation and other factors. This is again connected with the fact that variables are allowed to change in time and along the contours and we do not have to keep contours impermeable to fluid particles.

The cost of these advantages is, as we already mentioned above, the interpolation procedure between moving contours and regular grid. It is associated with some loss of accuracy and spurious viscosity, as well as some computational burden, but these losses appear to be small and the computational gain seems to exceed the cost by far for continuous fields with many contours.

The model which we use in this work and results of which we will discuss in the next chapters is a relatively simple version of such semi-lagrangian integral technique, incorporating features mentioned above. We consider it as a prototype which utilizes only a fraction of the potential of such method, and further work is needed to build a more comprehensive model. Nevertheless, even this simple model produces rather interesting results which we are going to discuss in the next chapters and can be also used to study a variety of other oceanographic problems. 


\section{Chapter 5}

\section{Interaction of a baroclinic vortex}

\section{with a sloping bottom}

\subsection{Model setup and initial conditions}

To model the interaction of an upper-ocean eddy with a continental slope - type topography we shall consider an unbounded rectangular domain. In most of our calculations topography will be represented by a constant slope in the direction of $y$-coordinate and independent of $\mathrm{x}$. The shallowing part will be on the upper side of the top view of the domain (see figure 5.1a) and the deeper part - below. In such a configuration topographic Rossby waves will propagate to the left of the picture, which we shall refer to as the "westward" direction. The shallowing (upward) direction will be equivalent to a "northward" azimuth for a planetary beta-plane.

Our domain will not have any lateral boundaries. One of the advantages of an "integral" technique (in which velocities are obtained via the convolution with a Green's function) is that we do not have to impose any explicit boundary conditions at the edges of a domain (although we can do it if necessary). To simulate a process without lateral boundaries one only need to ensure that these edges are far enough from the dynamically important region in the middle. Waves will radiate toward the boundaries of a domain and one can employ various methods to prevent them from coming back. In our case the radiative end is on the left (and also upper and lower edges). Short topographic waves can have "eastward" group velocities and radiate to the right side of a vortex, although in our model such radiation appears negligible.

Our computational domain is finite and is approximately equivalent to the landscape shown in figure 5.1b rather than an infinite slope (the configuration of the edges of the domain is not very important and we actually try to reduce its influence). It consists of a strip of a uniform slope (in some of our numerical experiments we also introduce a slope with a curvature and topographic perturbations) bounded by adjacent strips of a flat bottom. The upper (shallower) strip can represent 
a shelf and the lower (deeper) one - an abyssal plain. But practically we consider density contours only at the slope area and the solution in the "shelf" and "abyss" is not accurate. In most of our calculations we try to reduce influence of the edges of the domain by using "sponge" and "moving grid" techniques, which we shall describe later in this chapter and in some more details in the Appendix 2.

The typical size of our grid is 100 cells in $\mathrm{x}$-direction and 30 cells in $\mathrm{y}$-direction. But each cell has a length in the $\mathrm{y}$-direction two times larger than in $\mathrm{x}$ (this can be easily changed), so the actual shape of the domain is 100 to 60 . The ocean depth (stretched) is typically 10 units (1 unit is the $\mathrm{x}$-size of one cell), so for the case of constant stratification the deformation radius is about $10 / \pi \approx 3.2$ units. If this is to approximate a typical mid-latitude deformation radius of about 30 $\mathrm{km}$ (let's make it 32 for convenience) then our individual cell has dimensions of 10 by $20 \mathrm{~km}$ and the whole domain - 1000 by $600 \mathrm{~km}$, which is not unreasonable for continental slopes. The number of moving contours is the same as the number of the grid points. This is by no means necessary or computationally optimal, but we made it so just for simplicity.

In figure $5.2(\mathrm{a}, \mathrm{b})$ we show a test example of topographic waves dispersion from a localized initial disturbance (without a vortex) as it is represented in our model. The initial disturbance was a bell-shaped displacement of density contours placed between the center and the right end of the domain. Figure 5.2(a) shows the mesh plot of contours displacement (that is the displacement at a given point is proportional to the height of the mesh plot) after four periods of short topographic Rossby waves. The direction of the view is from the lower-left end of the domain. One can see rather complicated combination of dispersed and reflected waves with a definite influence of the boundaries. Figure 5.2(b) shows a mesh plot of the evolution of the middle contour in this experiment (time axis is directed toward us and from left to right). One can see how a large initial disturbance is dispersed into waves with different wavenumbers, with short waves lagging behind as they propagate generally "westward" toward the left edge. The mesh plot used to describe this test experiment is optimal to show wave radiation, but to describe motion of a vortex we shall use a different type of plots - usually a top view of the domain.

This test experiment was done without any damping of the radiated waves. In other experiments we use a sponge-like relaxation procedure at the left end to reduce the artificial effect of wave reflection from the edges of the domain. Density contours are nudged toward their reference (unperturbed) positions. More specifically, their displacements from the reference position is simply multiplied by a certain coefficient slightly less than 1 . This coefficient varies smoothly from one gridpoint to another approaching gradually 1 towards the middle of the domain. Without such relaxation topographic waves tend to bounce off the left end of the domain and propagate backward (to the right) in the form of short waves with wavenumbers close to the Nyquist frequency of the 
surface vortex above the slope: top view

shallow

topogr. wave direction. ("westward")
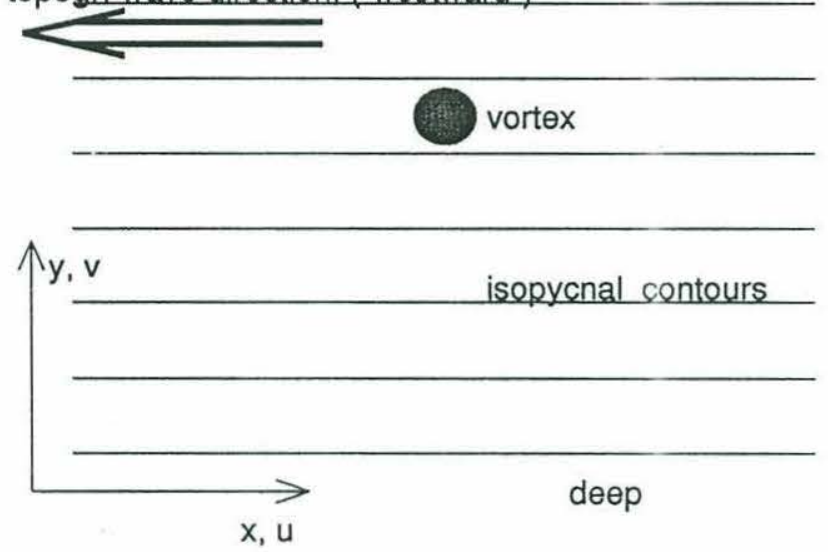

(a)

surface vortex above the slope
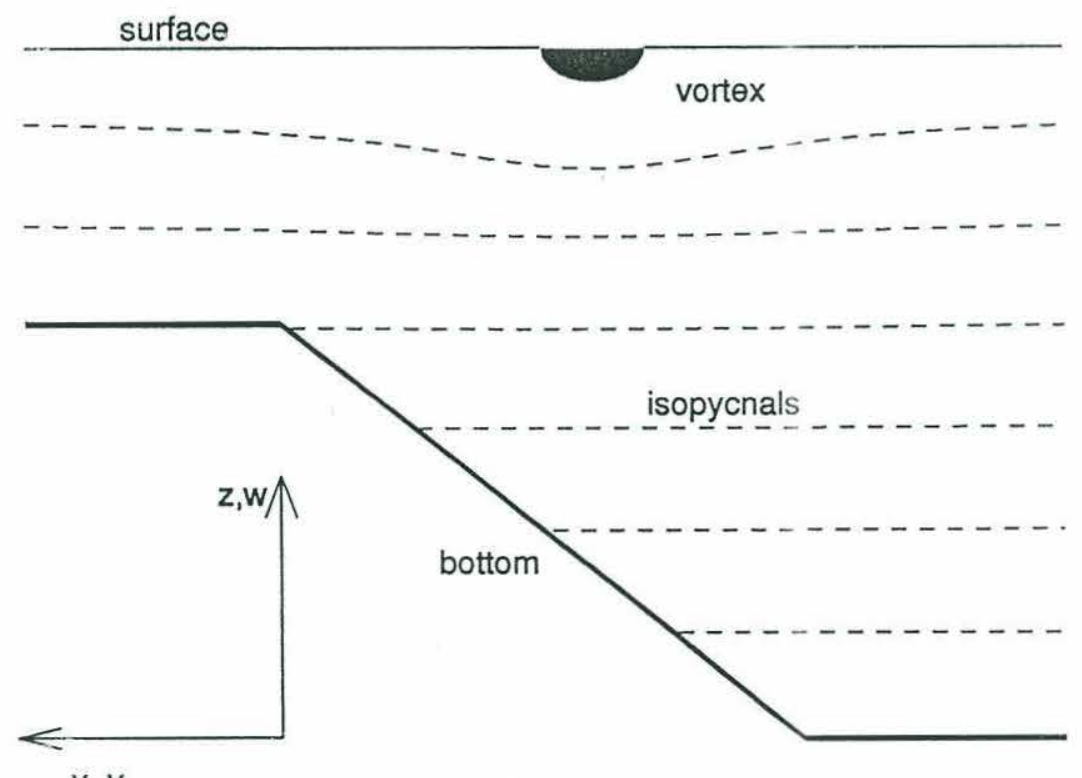

Figure $5.1(a, b)$. (a) Top view of the domain with a vortex.

(b) Side view of the domain configuration. 


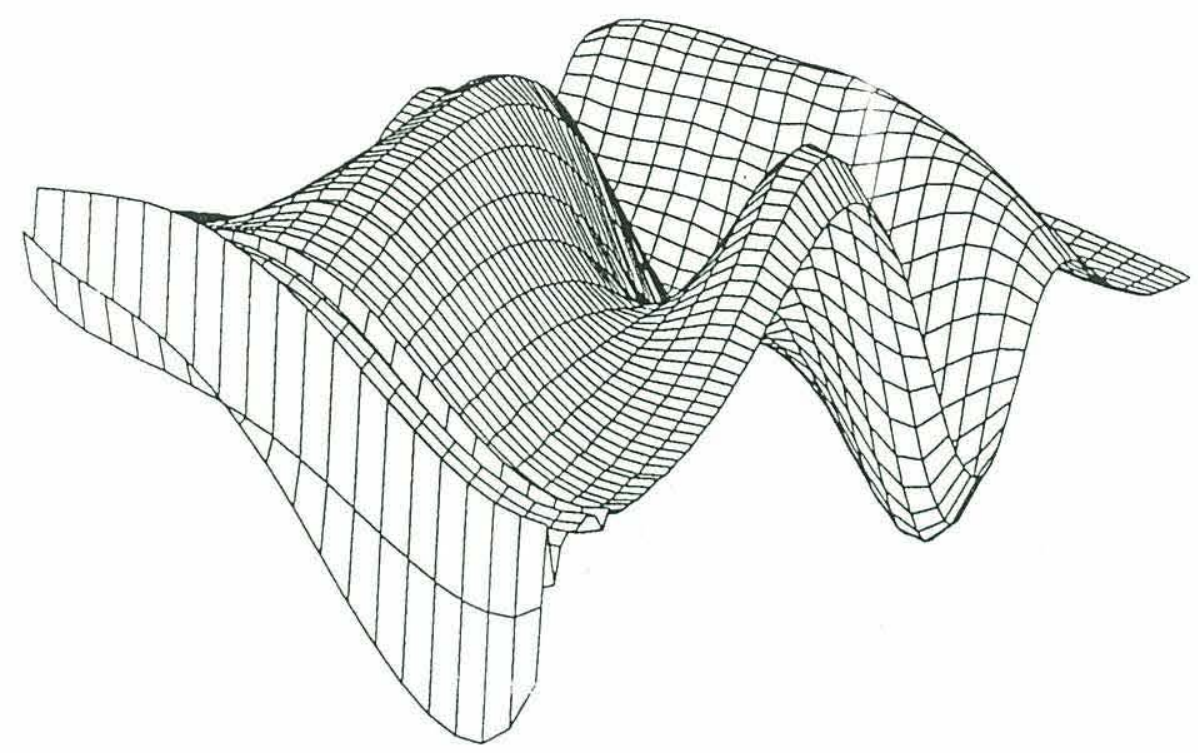

(a)

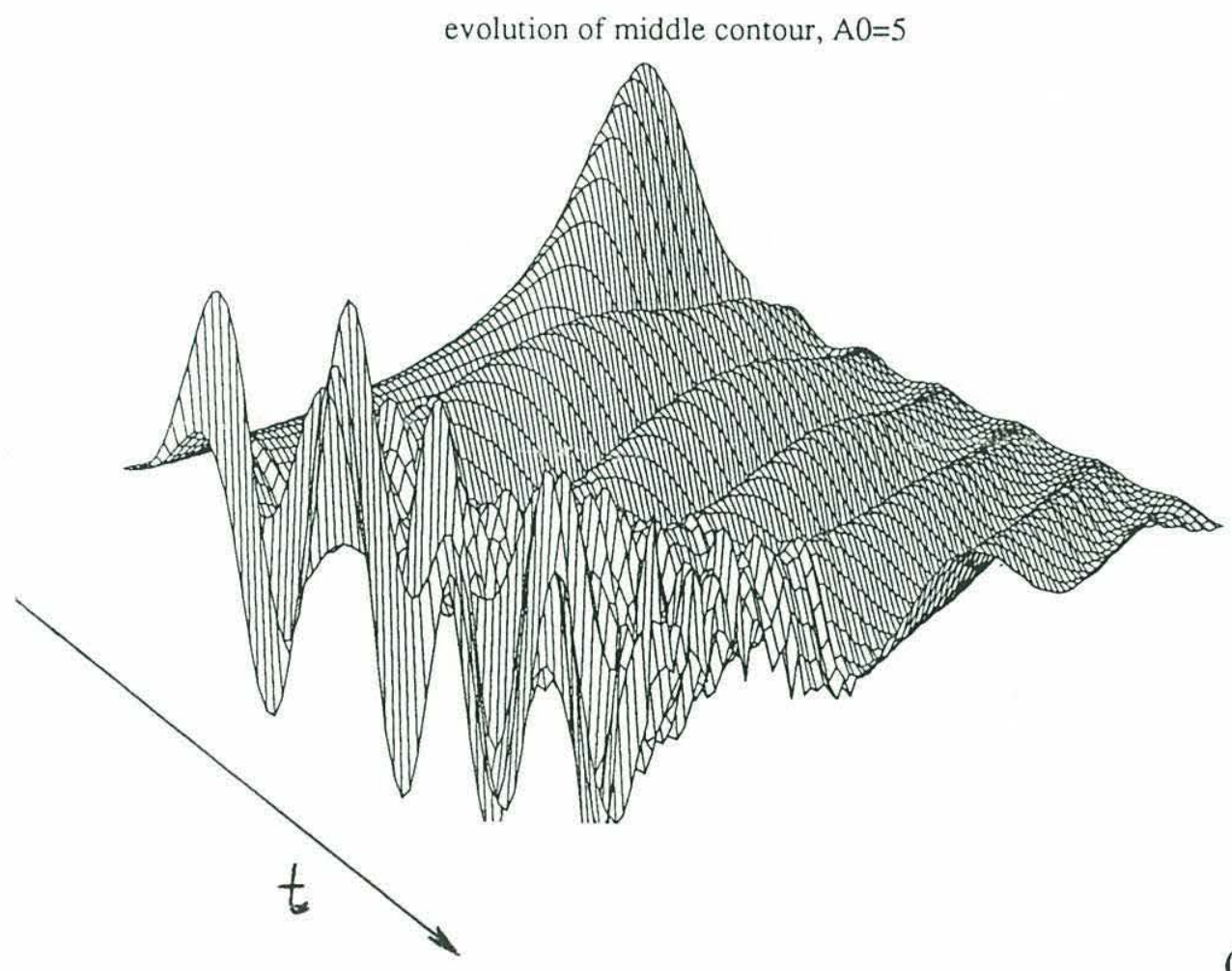

(b)

Figure $5.2(a, b)$. (a) Mesh plot of contour displacements in the wave dispersion test.

(b) Evolution of the middle contour in the test experiment; time axis is directed towards us and slanted from left to right. 


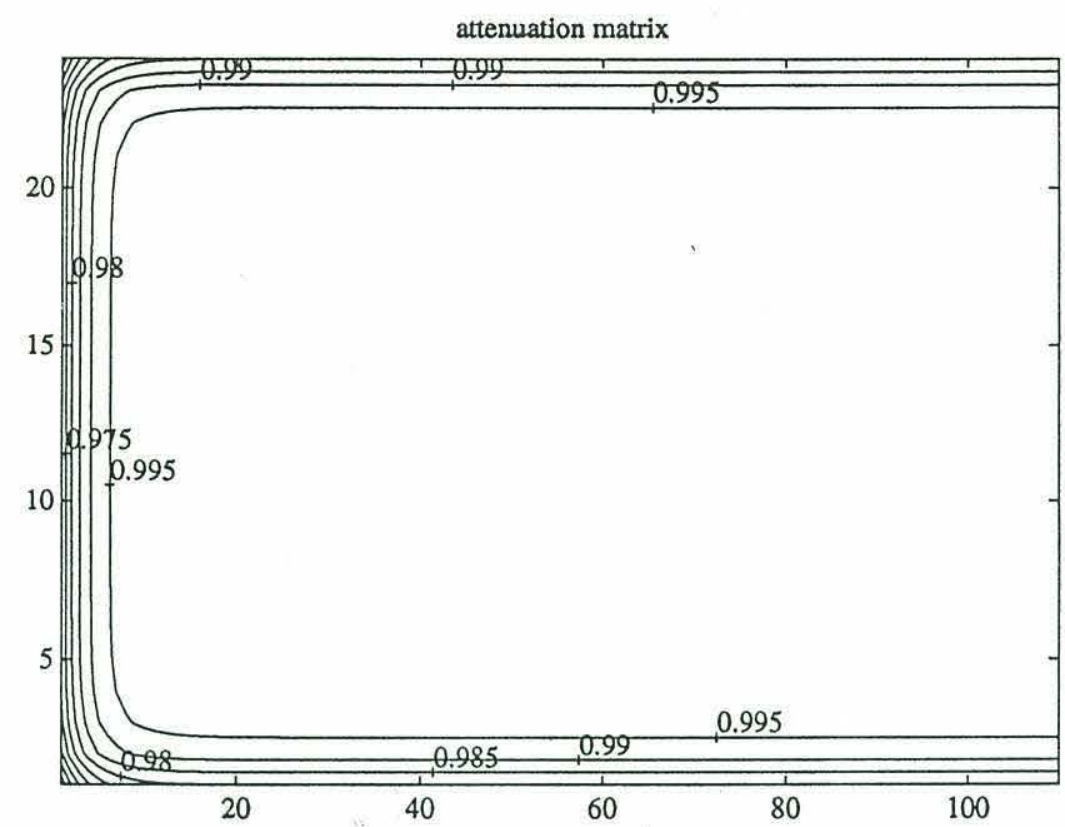

Figure 5.3 Typical "sponge matrix" for displacements of density contours.

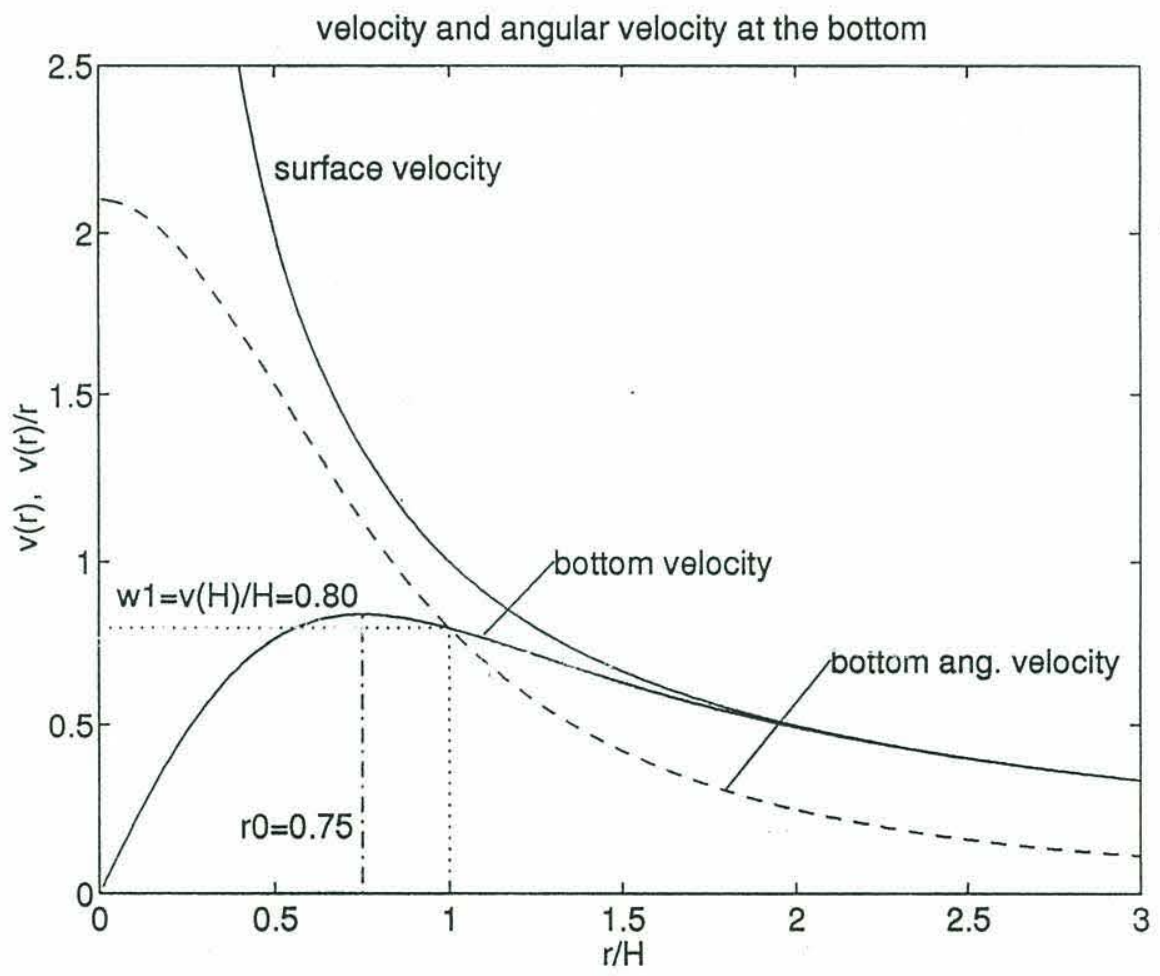

Figure 5.4. Velocity and angular velocity from a surface point vortex 
grid (this is seen clearly in both figures 5.2). But fairly small amount of relaxation (we use a damping coefficient of the order 0.96-0.98 at the very left end of the domain) is capable of suppressing them. A typical "sponge matrix" of damping coefficients as it varies from one point to another is shown in figure 5.3.

A similar relaxation is also used on the upper and lower side, although it is weaker than at the left end of the domain (see again figure 5.3). In addition to that we also make some artificial attenuation of a density perturbation at the upper and lower side when these edges are not wellcovered by density contours. This is needed because sometimes there are not enough density contours in these regions and when some grid points are beyond the interpolation range of these contours the mapping (in this case extrapolation) procedure can be inaccurate and should be adjusted to reduce the influence of these regions on the velocity field.

In the Appendix 2 we present some more examples of waves dispersion in our model and compare evolution of waves packet with theoretical calculations to check the accuracy of the numerical procedures. Also we present the dispersion of initial wave perturbation with a sponge layer, which can be compared to the figure 5.2 (without the sponge).

A baroclinic vortex in most of our numerical experiments will be represented by a point one, which is a three-dimensional potential vorticity anomaly at the surface or in the bulk of an ocean. This is a reasonably good approximation when the horizontal scale of a vortex is not larger than a "stretched" depth of an ocean (which is roughly a deformation radius multiplied by a factor of $\pi$ ). For mid-latitude lower continental slope regions this is typically $50-100 \mathrm{~km}$. So the point vortex approximation holds marginally for Gulf Stream or Gulf of Mexico rings and most of the smaller eddies. Later, in chapter 6 we shall compare results in a similar setting for a point vortex and a finite-volume one, which will be represented in our model by a patch of a uniform PV anomaly.

Intersections of isopycnal contours at the bottom in most of our experiments with vortices are at their reference positions initially. This is actually not a trivial assumption for initial conditions. Because of the presence of a vortex isopycnal surfaces generally should be displaced from their reference positions everywhere in the domain, including the bottom, (at least in the QG approximation). But the bottom boundary condition gives us an additional degree of freedom and one can impose such initial density perturbation at the bottom that it will compensate for the perturbation induced by a vortex (see figure $5.1 \mathrm{a}, \mathrm{b}$ ). So the total density anomaly at the bottom will be zero at the beginning of all our numerical experiments discussed below. This is of course not the most realistic initial condition, but not an unreasonable one, and calculations starting from different contour positions show a very similar behavior after the initial adjustment and wave radiation. 


\subsection{Scaling and nondimensionalization}

In the QG approximation a velocity field can be obtained if a PV distribution in the interior of the domain and a density anomaly at the upper and lower boundaries are known.

In the absence of these boundaries an azimuthal velocity from a point vortex is radially symmetric and is equal to

$$
\mathrm{v}=\mathrm{I} \frac{\mathrm{r}}{\mathrm{R}^{3}}
$$

where $\quad r^{2}=x^{2}+y^{2}, \quad R^{2}=r^{2}+\tilde{z}^{2}, \quad x, y, \tilde{z}$ are the coordinates of the "observing point" (a vortex is presumed to be placed at the origin of the coordinate system).

In the above formula $\mathrm{I}$ is the "strength" of a vortex (the three-dimensional analog of a circulation) which is equal to a product of a vortex PV anomaly and its volume: I $=q V$ (we already used this notation in chapter 3). When the surface and the bottom are present we must add all "images" that are symmetric relative to the surface and the bottom to calculate velocity, as was described in chapters 3 and 4 . For a surface vortex an azimuthal velocity is given by the following expression:

$$
\mathrm{v}=2 \mathrm{I} r \sum_{\mathrm{i}=0}^{\infty}\left(\widetilde{\mathrm{H}}^{2}(1+2 \mathrm{i})^{2}+\mathrm{r}^{2}\right)^{-3 / 2}=2 \mathrm{I} \frac{\mathrm{r}}{\widetilde{\mathrm{H}}^{3}} \sum_{\mathrm{i}=0}^{\infty}\left((1+2 \mathrm{i})^{2}+\xi^{2}\right)^{-3 / 2}=\mathrm{I} \frac{\mathrm{r}}{\widetilde{\mathrm{H}}^{3}} \Omega(\xi)
$$

Here $\quad \xi=\frac{r}{\widetilde{H}}$ is a horizontal radial coordinate normalized by a "stretched" depth $\widetilde{H}$, $\Omega(\xi)=2 \sum_{\mathrm{i}=0}^{\infty}\left((1+2 \mathrm{i})^{2}+\xi^{2}\right)^{-3 / 2}$ is the nondimensional angular velocity at the bottom.

Figure 5.4 shows velocities at the surface and the bottom from a point vortex of unit strength and the nondimensional angular velocity function $\Omega(\mathrm{r} / \widetilde{\mathrm{H}})$. Velocities converge at large $r$ toward the barotropic limit $\sim 1 / \mathrm{r}$ and angular velocity $\Omega$ - toward $\sim 1 / \mathrm{r}^{2}$.

One can expect even before doing any numerical experiments that the interaction of a vortex with a bottom slope will involve two competing processes: a vortex will induce a density anomaly at the bottom and the anomaly will radiate "westward" and propel a vortex itself. Evidently this interaction will depend on the relative rates of these two processes: creation of density anomalies by a vortex and radiation of waves away. These relative rates can be measured as the ratio of the two frequencies: a swirl angular velocity of water parcels at the bottom around a vortex (which is 
characterized by the nondimensional angular velocity $\quad \Omega$ multiplied by the strength of a vortex) and the frequency of topographic Rossby waves. Thus the topographic wave frequency will be a natural scale for angular velocity and, correspondingly, for the vortex strength. For topographic waves frequency we shall choose its high-wavenumber limit $\omega_{\text {top }}=\alpha \mathrm{N}$, where $\alpha$ is the slope angle and $\mathrm{N}$ is a characteristic buoyancy frequency. Thus a nondimensional angular velocity from a vortex of the strength $\mathrm{I}$, scaled by the topographic waves frequency $\omega_{\text {top }}$ will become

$$
\frac{\omega(\xi)}{\omega_{\text {top }}}=\frac{\mathrm{v} / \mathrm{r}}{\alpha \mathrm{N}}=\frac{\mathrm{I}}{\alpha \mathrm{N} \widetilde{\mathrm{H}}^{3}} \Omega(\xi)=\frac{\mathrm{A}}{\widetilde{\alpha}} \Omega(\xi)
$$

where $\quad \mathrm{A}=\frac{\mathrm{I}}{\mathrm{f}^{3}}, \quad \tilde{\alpha}=\alpha \mathrm{N} / \mathrm{f}-\mathrm{a}$ "stretched" slope angle.

The parameter A is a nondimensional strength of a vortex, the same as the one we used in chapter 4 in our semi-analytical model. The combination $\mathrm{f}^{3}$ in the denominator is a natural scale of the "strength" which must be a product of vorticity and volume units. Because we do not have any other length scales in the case of a point vortex, it can be constructed only from the total depth of an ocean $\widetilde{\mathrm{H}}$ (in stretched coordinates).

Expression (5.3) still depends on the normalized radial coordinate $\xi=r / \widetilde{\mathrm{H}}$. One can have several options for a characteristic angular velocity scale: for example the maximum value of $\Omega(\xi)$ can be used or some intermediate value at a chosen $\xi$. We consider it natural to evaluate $\Omega$ at $\xi=1$, that is horizontal distance equal to a stretched depth of an ocean. We shall denote this value as $\Omega_{1}=\Omega(1)=0.80$. This value is considerably smaller than the maximum value of $\Omega$ (at $\xi=0$ ), which is equal to $\sim 2.09$ (see figure 5.4) but we consider it more relevant to include the characteristic lengthscale of a system, which is the "stretched" depth $\widetilde{\mathrm{H}}$.

With this choice of the angular velocity scale we can make another, more convenient measure of a vortex strength. We shall introduce a new nondimensional amplitude $\widehat{A}$ which is equal to the above mentioned ratio of two frequencies:

$$
\widehat{\mathrm{A}}=\frac{\mathrm{v} / \mathrm{r}}{\omega_{\text {top }}}=\mathrm{A} \frac{\Omega_{1}}{\tilde{\alpha}}=\mathrm{A} \frac{0.8}{\tilde{\alpha}}
$$

The parameter A characterizes the strength of a vortex but does not include a slope angle. But the nondimensional amplitude $\widehat{\mathrm{A}}$ is a function not only of the vortex actual "strength" but also the depth of the ocean $\widetilde{\mathrm{H}}$ and the slope angle $\tilde{\alpha}$. When $\widehat{\mathrm{A}}=1$ the swirl angular velocity at the distance $\mathrm{r}=\widetilde{\mathrm{H}}$ from a vortex is equal to the frequency of short topographic waves. 
This measure $\widehat{A}$ will be used in our numerical experiments to characterize the vortex amplitude. One can expect that when is $\widehat{A} \ll<1$ perturbations induced by a vortex will be quickly radiated away in the form of almost linear waves. Nonlinearity will increase as $\widehat{A}$ approaches unity. At $\widehat{\mathrm{A}} \sim 1$ one can expect a rather delicate balance between rotation induced by a vortex and wave radiation with increasing probability of wave breaking at $\widehat{A}$ larger than unity. At very large amplitudes, $\widehat{A} \gg 1$ water parcels can be rotating around a vortex in almost circular trajectories and only weakly influenced by the wave radiation. This is the case, for example for many numerical experiments with a strong vortex on the beta-plane (McWilliams and Flierl, 1979, Mied and Lindeman, 1979, and other studies). Therefore, this amplitude parameter $\widehat{A}$ is in some sense equivalent to a Richardson number for stratified flows which is the ratio of a flow shear (the wavebreaking tendency) and a buoyancy frequency (the restoring force and the wave-radiating tendency). The Richardson number plays a crucial role in the internal wave dynamics and mixing processes and one can expect similar importance of a nondimensional amplitude $\widehat{A}$ in our case. We shall have more comments on it later when we shall discuss our numerical results and introduce a concept of a wave-breaking and a wave-gliding regime in comparing vortex motion on a planetary beta-plane and over a sloping bottom. We shall argue also in chapter 7 that the difference between the results of our model and some numerical experiments in a two-layer case can be attributed to the different representation of this breaking-radiation balance for layered and continuous stratification.

Now we shall introduce a velocity scale which will be used in presenting and describing the output of our model. One obvious choice for the velocity measure is simply $\Omega_{1} \widetilde{\mathrm{H}}$ which is based on the scale for the angular velocity. But we shall use a slightly different value, based on the concept of a "hetonic" translation mechanism which we shall introduce in section 5.5. The reason for this is that we want a scale for the translational velocity of a vortex itself, not for the swirl velocity of surrounding fluid parcels. As we shall see below, the vortex is propelled by a density anomaly at the bottom which has an equal in magnitude and opposite-signed to a vortex circulation. This anomaly is misaligned with a vortex horizontally and the whole structure thus resembles a "heton" (vertically tilted dipole). The translational velocity of a vortex can be compared to the maximal velocity possible for the heton structure. This maximal possible velocity will be obtained when an opposite-signed bottom vortex is placed at the point where the bottom velocity from a surface vortex reaches maximum. Because of the vertical symmetry it means that the maximum surface velocity from a bottom vortex will be reached at the position of a surface vortex itself. This maximum velocity $\mathrm{v}_{0} \approx$ 0.84 (from a vortex of unit strength $\mathrm{I}$ and with a unit depth $\widetilde{\mathrm{H}}$ ) is reached at $\mathrm{r}_{0} \approx 0.75 \widetilde{\mathrm{H}}$ (see again figure 5.4) and $\mathrm{v}_{0}$ will be accepted as our velocity scale. It can be called the "optimal heton" translation velocity. The ratio $\quad v$ of a translational velocity $v_{t r}$ to the scale $v_{0}$ can be interpreted as an efficiency of this "hetonic" mechanism: 


$$
v=\frac{v_{\text {tr }}}{v_{0}}, \quad v_{0}=v(0.75 \widetilde{H}) \approx 0.84 \frac{\mathrm{I}}{\widetilde{H}^{2}}
$$

Finally, time itself will be scaled by the short topographic waves period:

$$
\tau=\frac{\mathrm{t}}{\mathrm{T}_{\text {top }}}, \quad \mathrm{T}_{\text {top }}=\frac{2 \pi}{\widetilde{\alpha} \mathrm{N}}
$$

where $\tau$ is nondimensional time which we shall use in our experiments.

\subsection{Initial evolution of a vortex}

When we start our numerical experiments from unperturbed (at the bottom) density contours the initial velocity field at the bottom will look like the one sketched in figure 5.5 . Initially straightline density contours will start to be displaced around a vortex antisymmetrically: to the right of a (cyclonic) vortex in a top view they will be pushed upslope into shallower water and to the left - into deeper water. An upward displacement will induce a positive density anomaly and correspondingly a negative (anticyclonic) vorticity, which is equivalent to the squashing of vortex tubes in a layer model; on the left side a region of cyclonic vorticity will develop. Thus the initial density anomaly will lead to a dipolar vorticity structure which will push a cyclonic vortex upslope (see figure 5.6). For an anticyclonic vortex the situation is opposite and it will move initially in a downslope direction. In both cases (cyclones and anticyclones) opposite-signed vorticity is induced to the right of the vortex, and like-signed - on the left side. This process is analogous to initial evolution of a vortex on a planetary $\beta$-plane, where it should also move meridionally, cyclonic vortices - to the north and anticyclones - equatorward. While this process is not well-documented for oceanic vortices on the planetary $\beta$-plane, it is very conspicuous in the atmospheric dynamics, where strong tropical cyclones propagate rapidly northward after formation.

This emerging dipolar structure at the bottom under the vortex immediately starts radiating topographic waves toward the left edge of the domain. Because these topographic waves propagate 
velocity at the bottom from a surface vortex

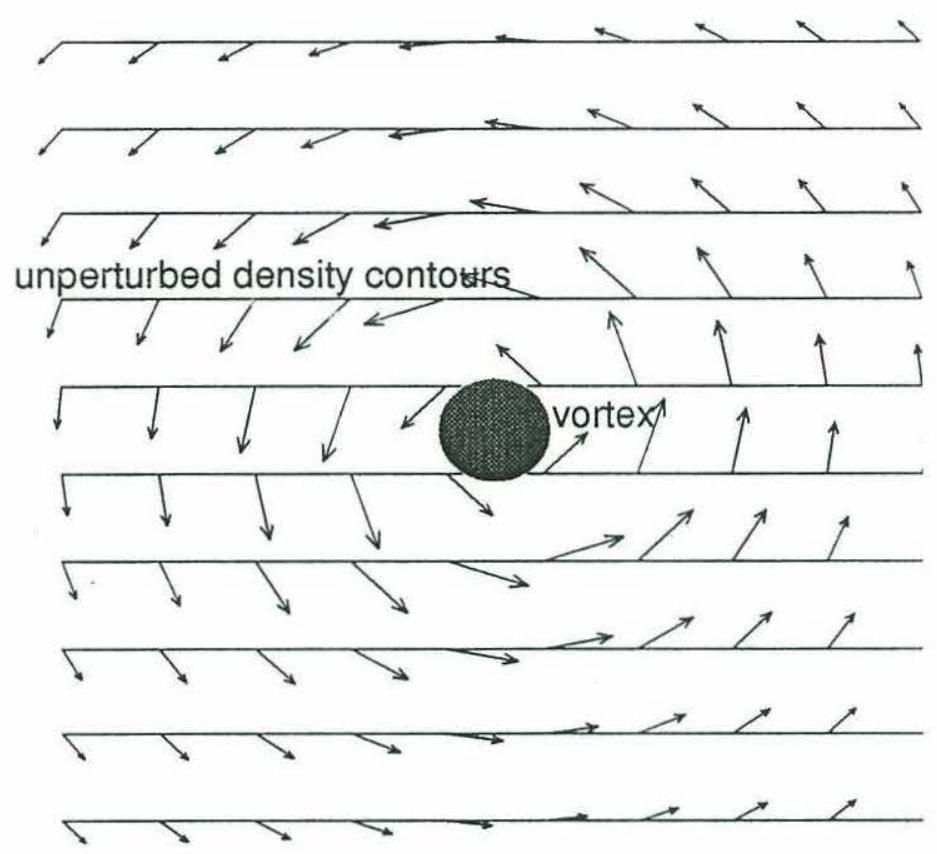

Figure 5.5. Velocities at the bottom with unperturbed density contours.

initial dipolar structure at the bottom

top view

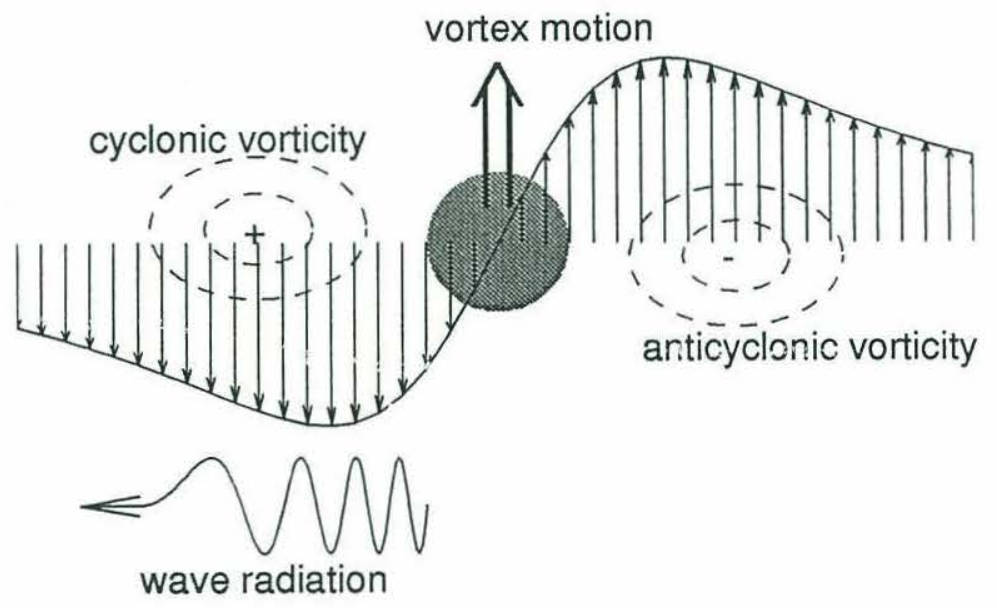

Figure 5.6. Initial dipolar structure in the vorticity field at the bottom. 


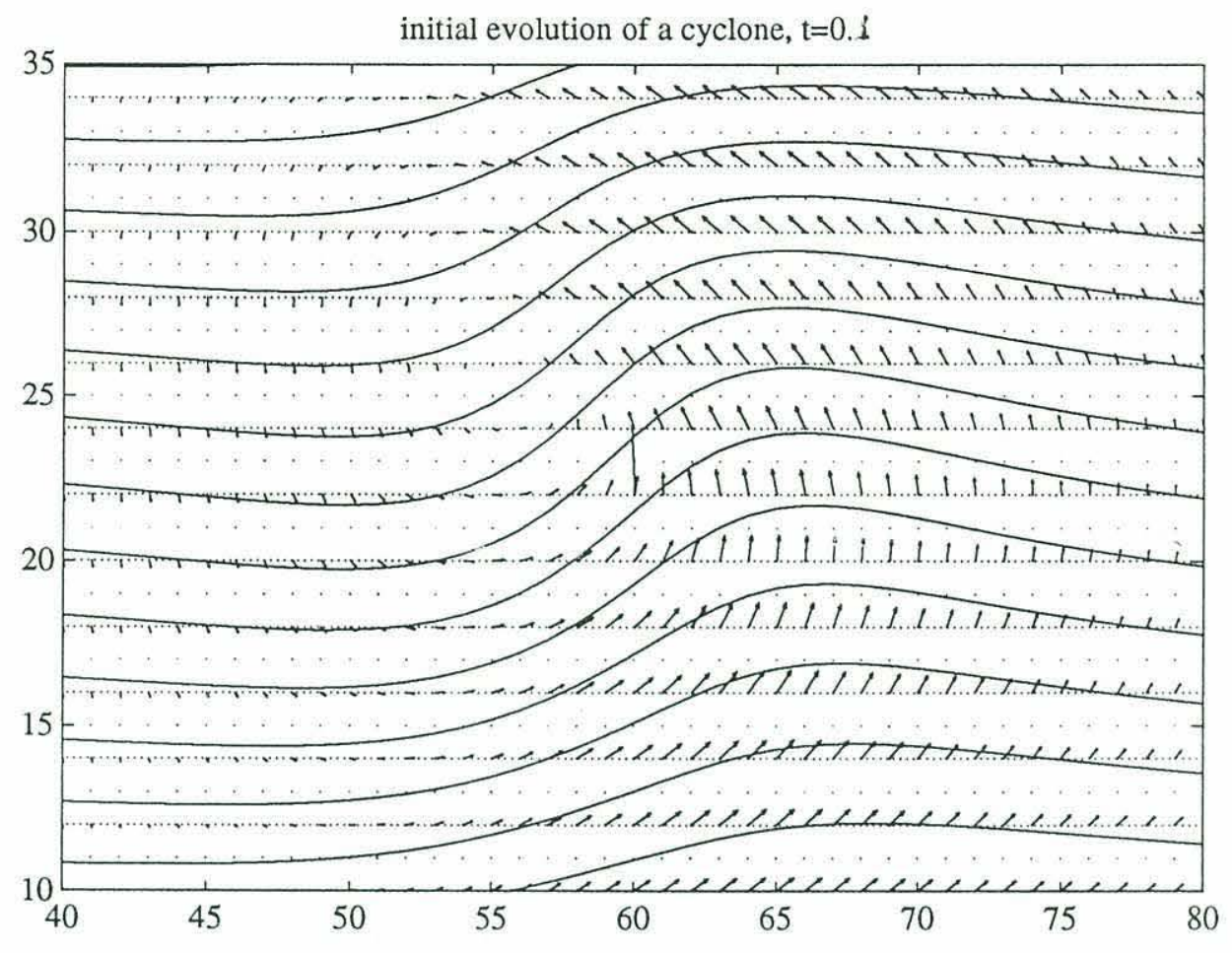

(a)

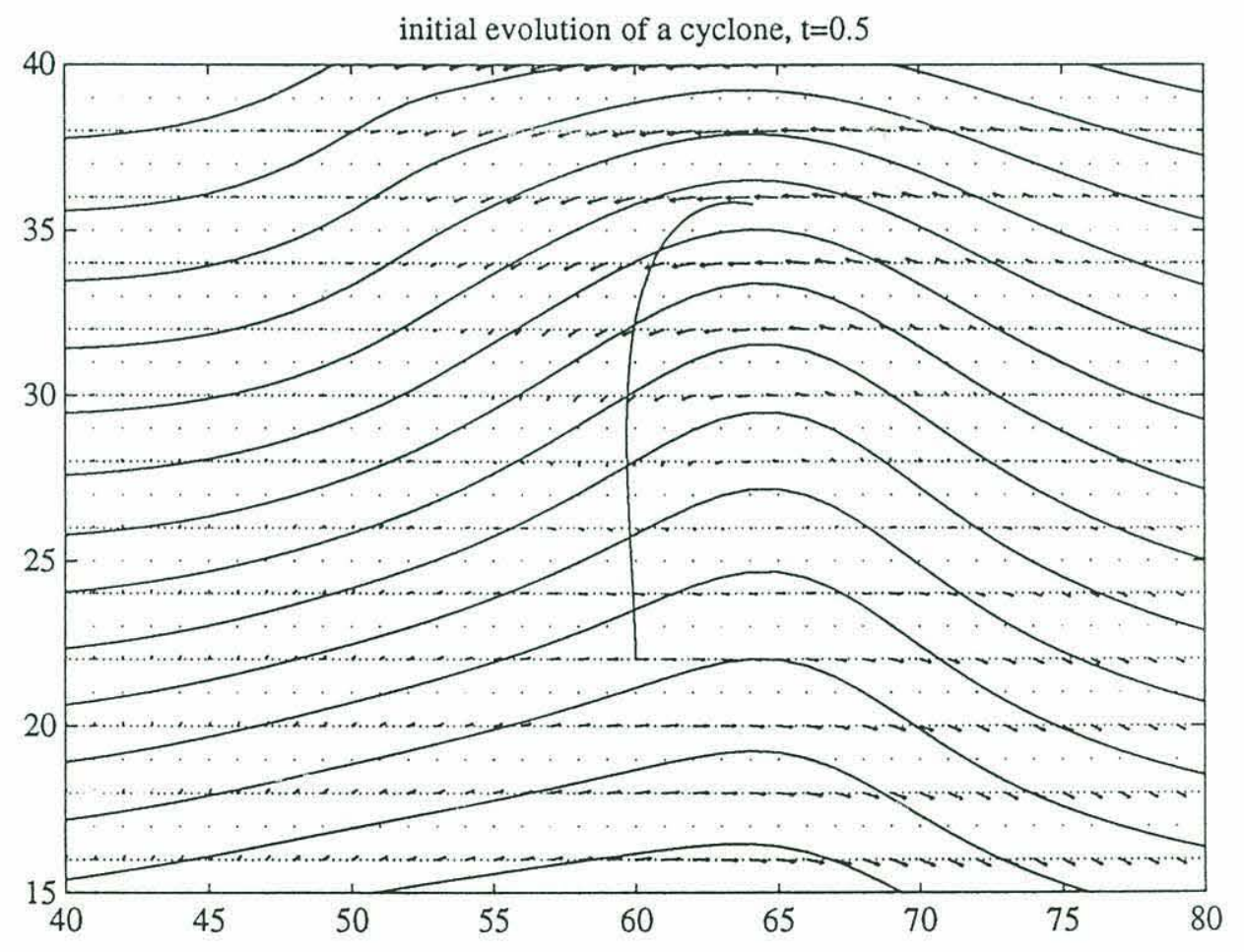

(b)

Figure $5.7(a, b)$. Displacement of density contours and a velocity field at the early stage of experiment 
generally "westward" - to the left of the upslope direction - the whole structure tends to be shifted to the left of the vortex while being deformed because of the dispersion and the vortex velocity field. Long waves will go faster than short ones and thus the displacement of contours to the left of a vortex is smoother and smaller in amplitude while at the right side will be steeper and larger. This is similar to the "nonlinear tendency" concept introduced by Smith and O'Brien (1983) to explain meridional (upslope) motion of a vortex, which we mentioned in chapter 1 (see figure 1.3). This initial left-right asymmetry is clearly seen in figure 5.7(a) where density contours and the bottom velocity field are plotted for a cyclonic eddy after a time $\tau=0.1$ (time unit is a period of short topographic Rossby waves). Generally velocities on the right side are larger and more concentrated near the vortex itself, while to the left of the vortex density and velocity fields are smoother but decay less rapidly away from the vortex.

Another simplified way to look at this left-right asymmetry is to assume that the initial dipolar structure moves as a whole to the left relative to the vortex itself, while the velocity field of the vortex remains practically constant in time. Then the part of the dipolar structure which was initially to the right of the vortex and was displaced upslope will appear on the left side of it where its velocity is downward (for a cyclone) so it counteracts its initial displacement and will diminish its amplitude. The part which was initially to the left of the vortex fill go further to the left from it so the displacement of density contours to the left of the vortex will be spread over a larger area. On the other hand the part which was initially further to the right of the vortex will still be on its right side and the displacements of contours there will continue to grow. The result will be that on the right hand side the contour displacements will have larger amplitude while on the left-hand side they will be smaller but spread over a larger area.

The vortex moves initially with constant acceleration because the amplitude of the dipolar structure grows linearly with time until wave radiation effects begin to be important. After a time of order of a topographic wave period the like-signed vorticity anomaly which was initially on the left side for both cyclones and anticyclones is mostly radiated away. The opposite-signed perturbation which was initially on the right side reachs its maximum and appears approximately under the vortex. Still it is always situated on the right-hand side, because this opposite-signed perturbation is constantly created by the vortex velocity field. If a vortex itself is unmoving this density anomaly would be symmetrical in the north-south direction. Because of the vortex motion this symmetry is slightly perturbed. Typically the maximum of the density anomaly is ahead of the vortex motion, while the centroid trails slightly behind it. This induces a small "eastward" component of velocity in the presence of a surface. Without the surface a vortex moves in almost cross-slope direction with a very small "westward" component. Still the along-slope component is not dominant in comparison with the cross-slope one. 
Before reaching a steadily translating state the vortex motion undergoes some oscillations and adjustment. At the time approximately half of the short wave period the meridional (cross-slope) component of velocity reaches its minimum and the cumulative vorticity anomaly at the bottom - its maximal opposite-signed value. Then, as the vortex velocity reaches its minimum, the situation has a certain resemblance to the initial position (unmoving vortex), although density contours are already significantly perturbed. The vortex again acquires some meridional acceleration and the process nearly repeats itself but the amplitude of oscillations decreases rapidly as adjustment takes place. Finally, at approximately $\tau=2$ the vortex reaches the near-steady state in which it moves in the almost cross-slope direction. This steady translation will be the subject of the next section.

\subsection{Later stage: steady motion}

After the initial adjustment and a wave surge which lasts approximately one or two of our nondimensional time units (period of short topographic Rossby waves, $2 \pi / \alpha \mathrm{N}$ ) vortex motion reaches a near-steady state. In this state a vortex translates due to the interaction with a bottom density anomaly induced by its own velocity field.

Although real continental slopes have a finite width and the influence of their edges can be important, we consider it useful to understand the behavior of a vortex over a slope in its "pure" form. Because a vortex moves quite rapidly it soon approaches the edges of our computational domain. When a vortex comes close to the edges of the model it is influenced by them and steady motion is not completely achieved. To reach a steady translation in our model (if there is such a dynamical regime) we introduced a moving grid which propagates with a vortex itself. Numerical implementation of the moving grid technique in our case is described briefly in the Appendix 2. Trajectories of the grid itself will be shown in the figures together with trajectories of vortices and positions of isopycnal contours.

The initial evolution of a vortex from the state with unperturbed isopycnal contours can be considered as a superposition of a steady translation with the corresponding density anomaly structure and the decaying oscillatory motion during the adjustment process in which the "leftover"

of an initial density anomaly (relative to the steadily translating state) is radiated away as topographic Rossby waves. This final equilibrium state appears to be independent of initial conditions and runs starting from a different initial bottom density structure show the same final translation after the adjustment process which lasts approximately two short wave periods. 
Surprisingly at the first glance, this steady motion is mainly in the meridional (cross-slope) direction) and this direction of motion does not depend significantly on the vortex strength.

Let us consider in some details the results of some of our numerical experiments with a point vortex over the uniform slope, shown in figures 5.8-5.11 (a,b). Upper pictures (a) show positions of density contours (solid lines) at a given time (typically at time $\tau=5$, well after the completion of the adjustment process) and a trajectory of a vortex itself. This trajectory often originates out of the domain because of the moving grid procedure. Reference positions of these contours are shown by dotted lines (in most experiments they are just straight horizontal lines). The type of topography is shown by the solid line at the left end of the plot (for uniform slope it is just a straight almost vertical line slanted to the right which means that the upper end of the plot is shallower). Also shown is the trajectory of the moving grid (scaled two times smaller). Positions of a vortex at integer time moments $(\tau=1,2,3 \ldots)$ are shown by small empty circles "o" (the same for the grid). Lower pictures (b) show density anomalies at the same moment of time (solid lines). Positive anomalies mean that contours are shifted upslope from their reference positions, negative downward. Positions of the vortices are again indicated by empty circles, also velocities of the vortices at the given time moment are shown by thick solid lines, with the lengths proportional to amplitudes. The centroid of a density anomaly is shown by a star "*". Its coordinates $\left(\mathrm{x}_{0}, \mathrm{y}_{0}\right)$ are naturally defined as first moments divided by the a zero moment of a density anomaly:
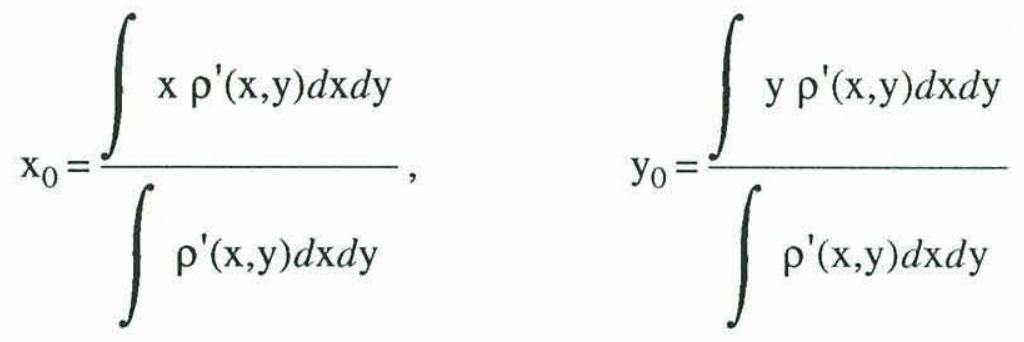

As one can see in most pictures centroids are displaced significantly from the maximum of a density anomaly, although these maxima are usually well localized. This happens because there is always a region of like-signed vorticity to the left of a vortex position (opposite sign for density anomaly) which leads to a large shift in the centroid position. In most experiments the line between the horizontal projection of a vortex position and a density anomaly centroid is approximately perpendicular to the velocity vector of a vortex. This indicates that vortex motion can be roughly interpreted as advection by an opposite-signed point vortex at the bottom situated at the position of a density anomaly centroid. 
surface cyclone, $\mathrm{Av}=0.4, \mathrm{tf}=5$

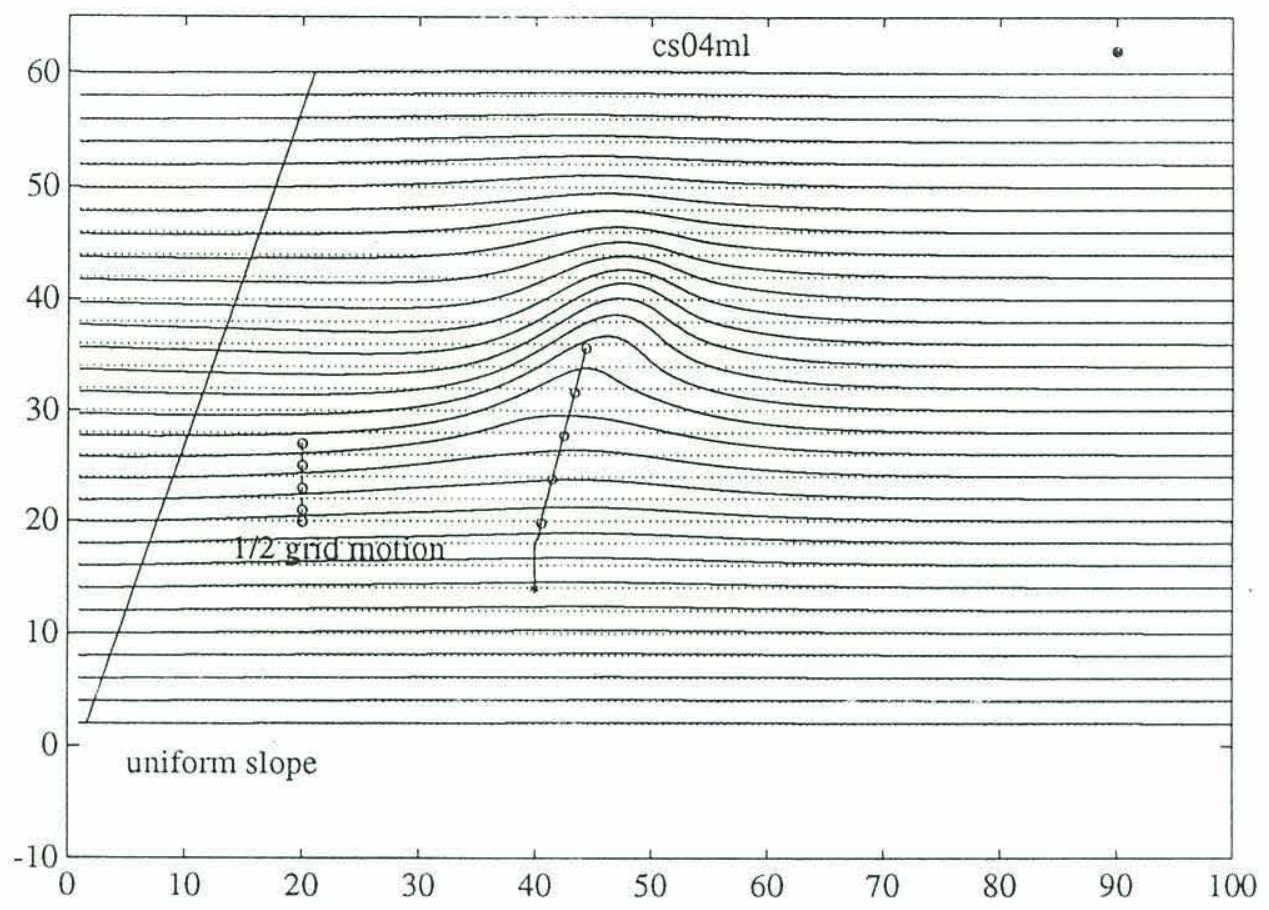

(a)

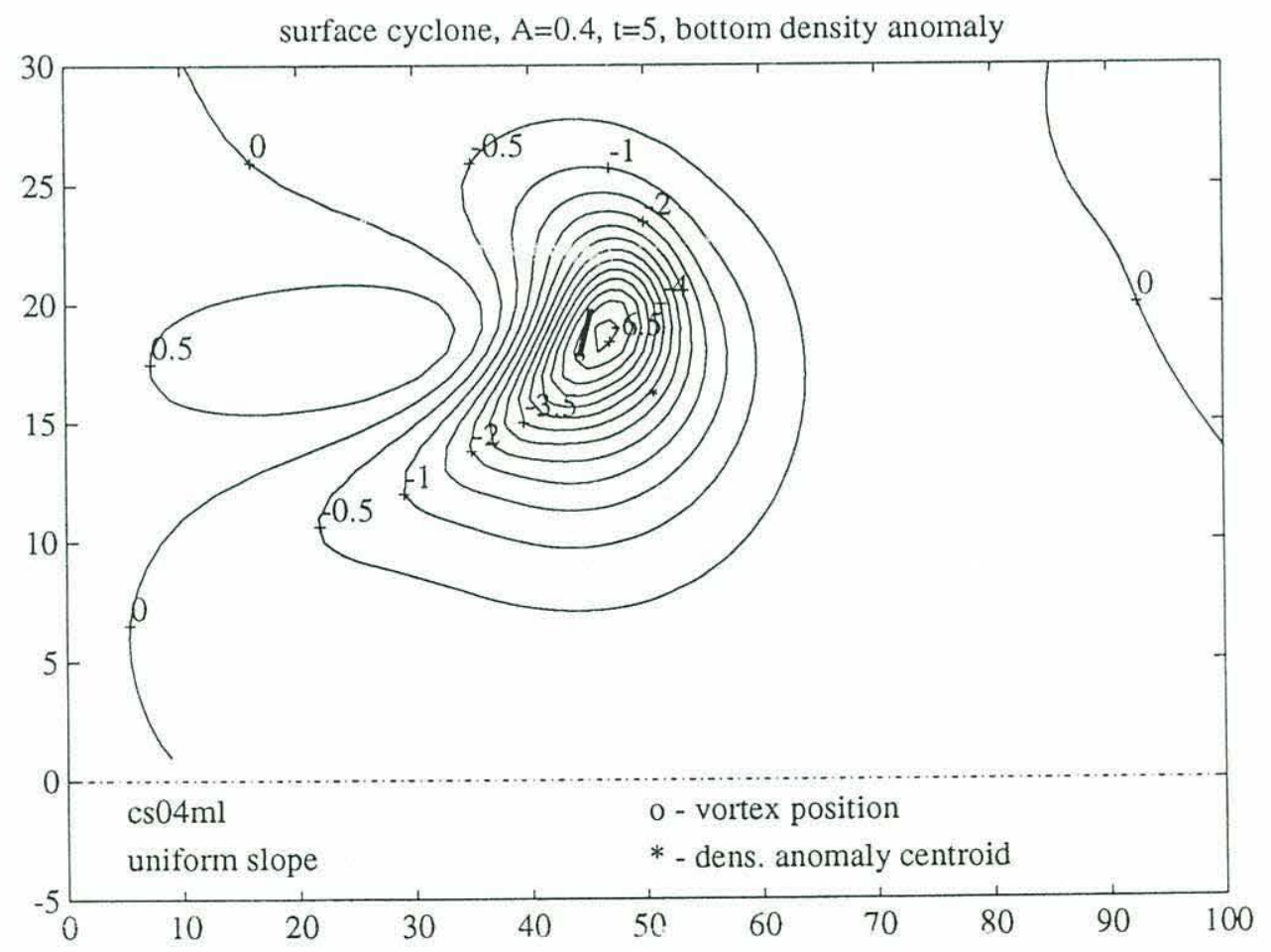

(b)

Figure $5.8(a, b)$. The vortex trajectory (a) and the bottom density anomaly (b) for the cyclonic vortex with $\widehat{A}=0.4$. 
surface cyclone, $A v=1, t f=8$

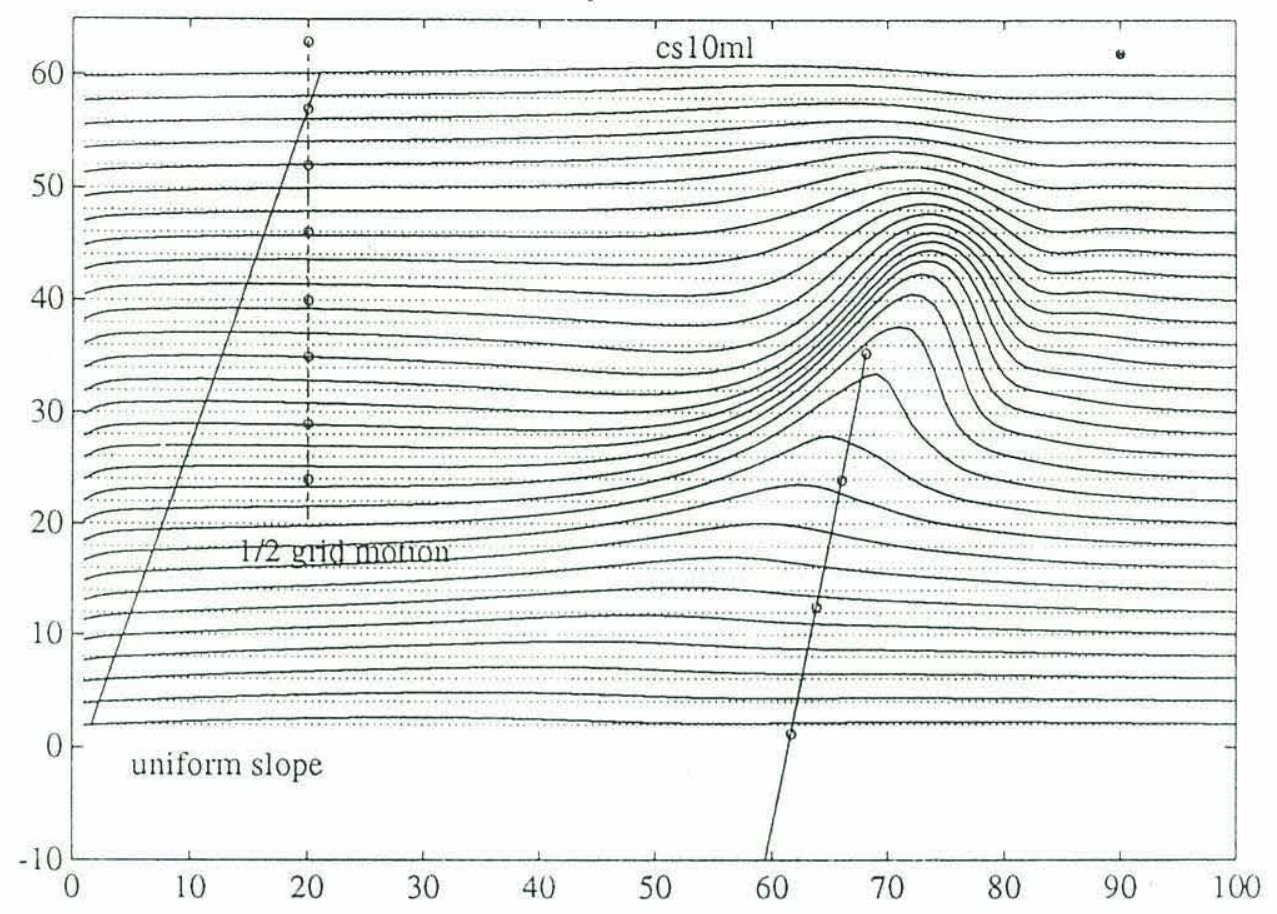

(a)

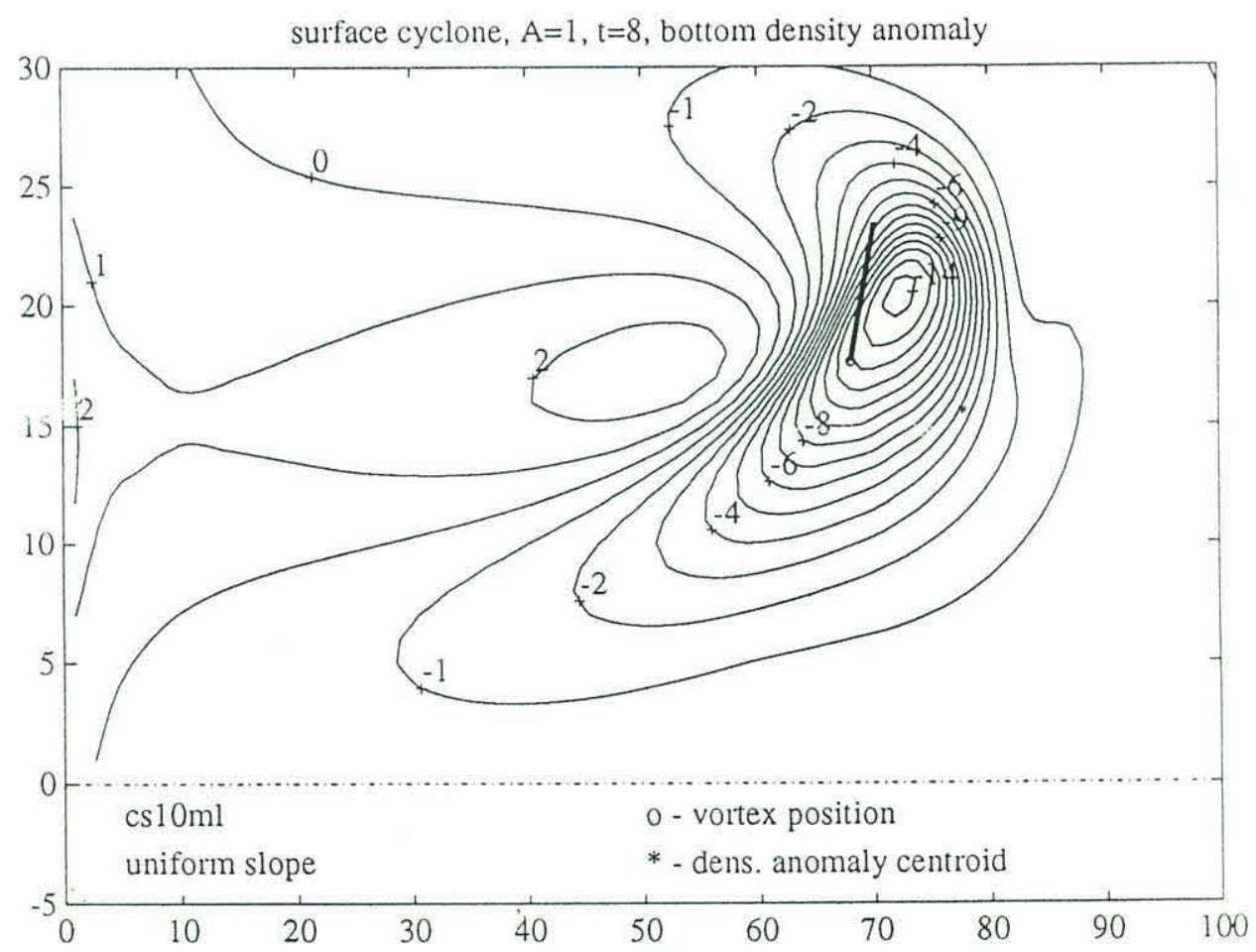

(b)

Figure $5.9(\mathrm{a}, \mathrm{b})$. Same as $5.8(\mathrm{a}, \mathrm{b})$ but for $\widehat{\mathrm{A}}=1$. 
surface cyclone, $\mathrm{Av}=1.5, \mathrm{tf}=5$

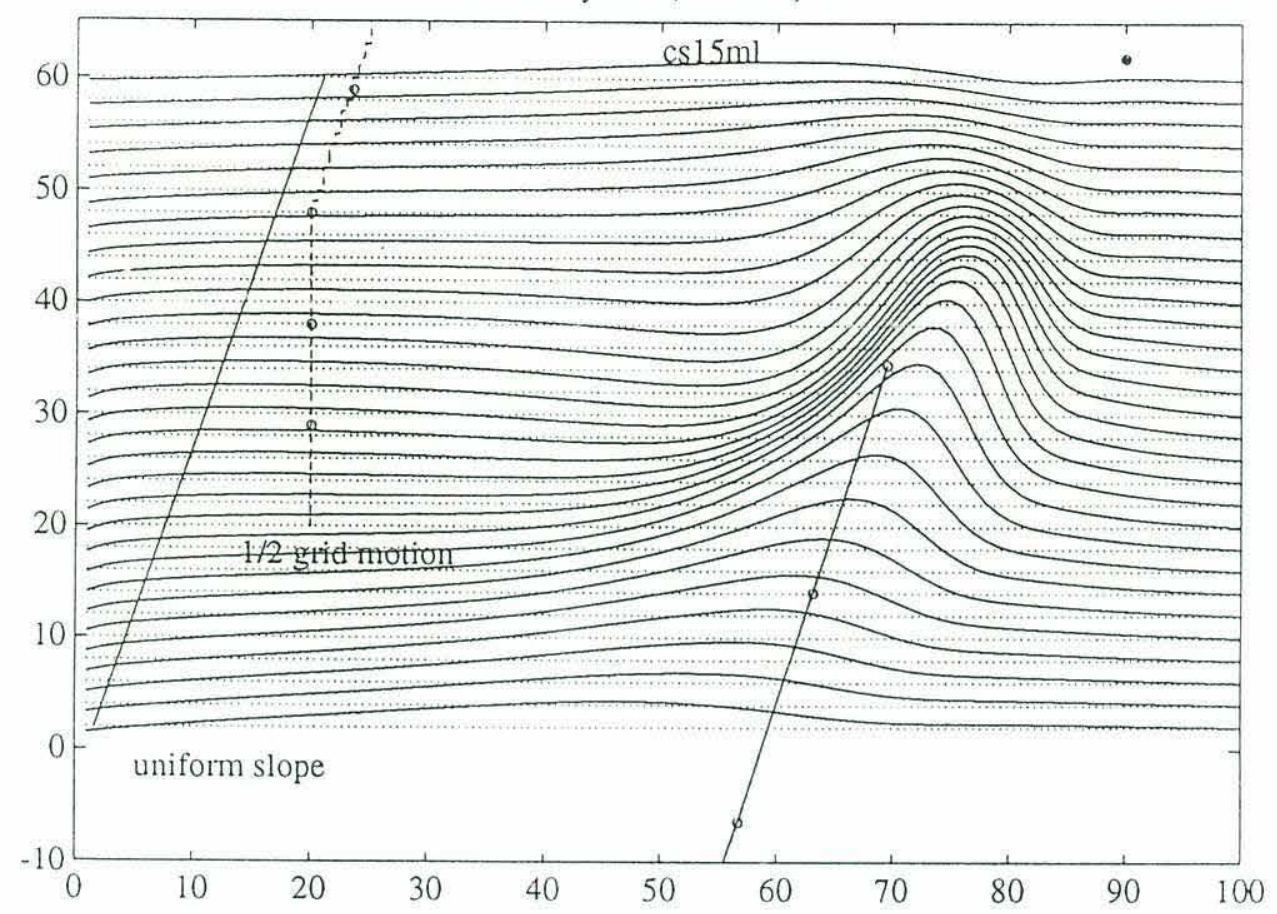

(a)

surface cyclone, $A=1.5, t=5$, bottom density anomaly

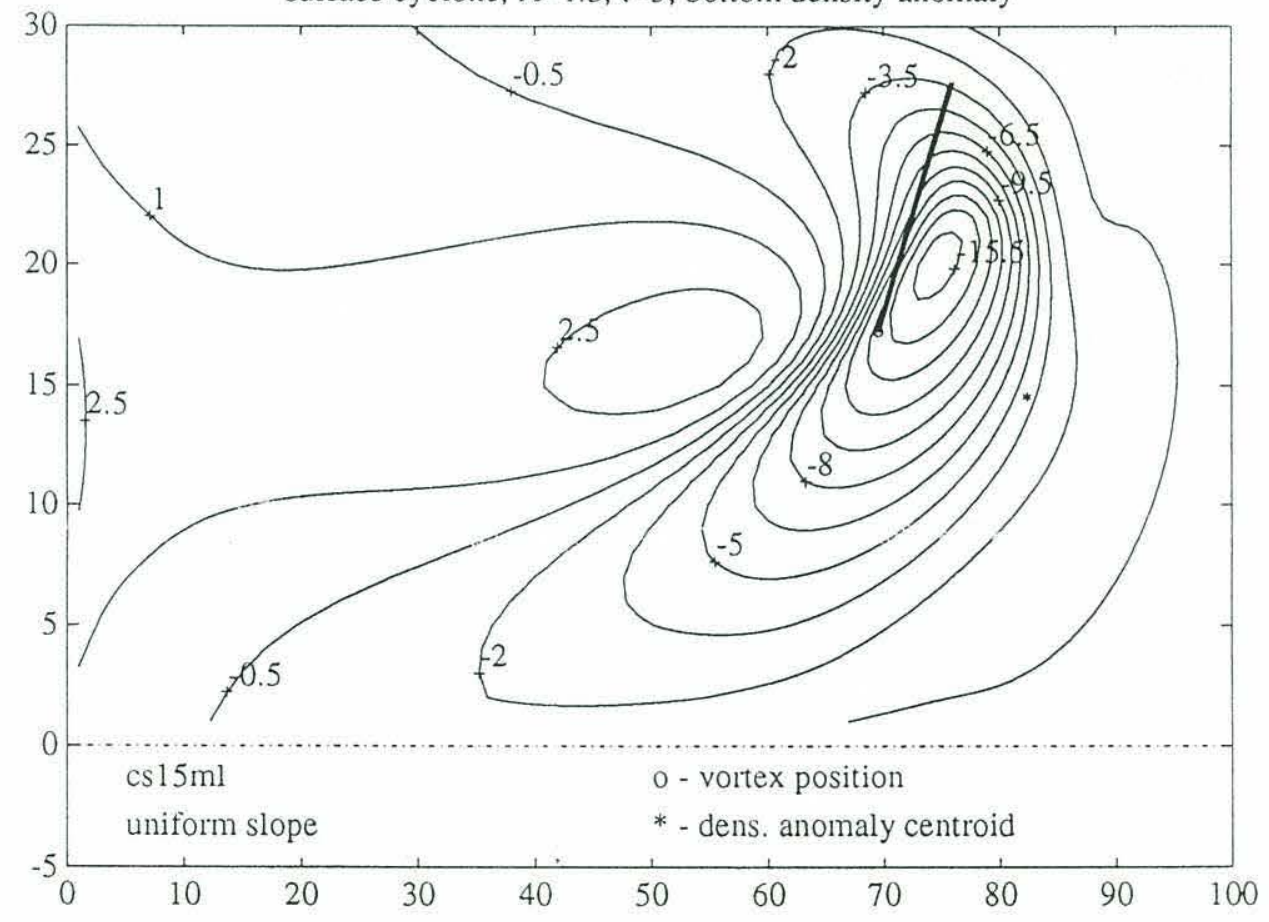

(b)

Figure $5.10(a, b)$. Same as $5.8(a, b)$ but for $\widehat{A}=1.5$. 
surface cyclone, $A v=0.6, t f=5$

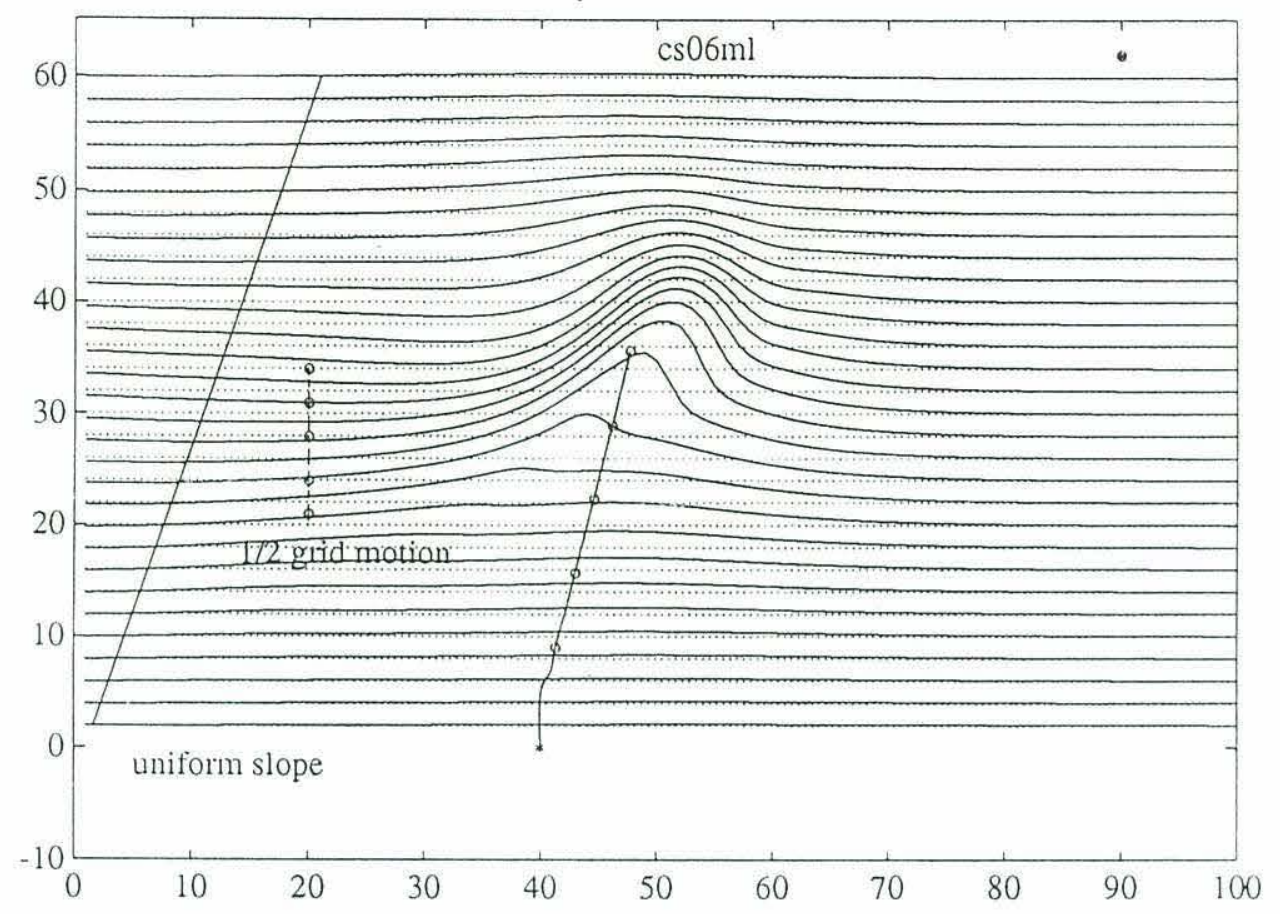

(a)

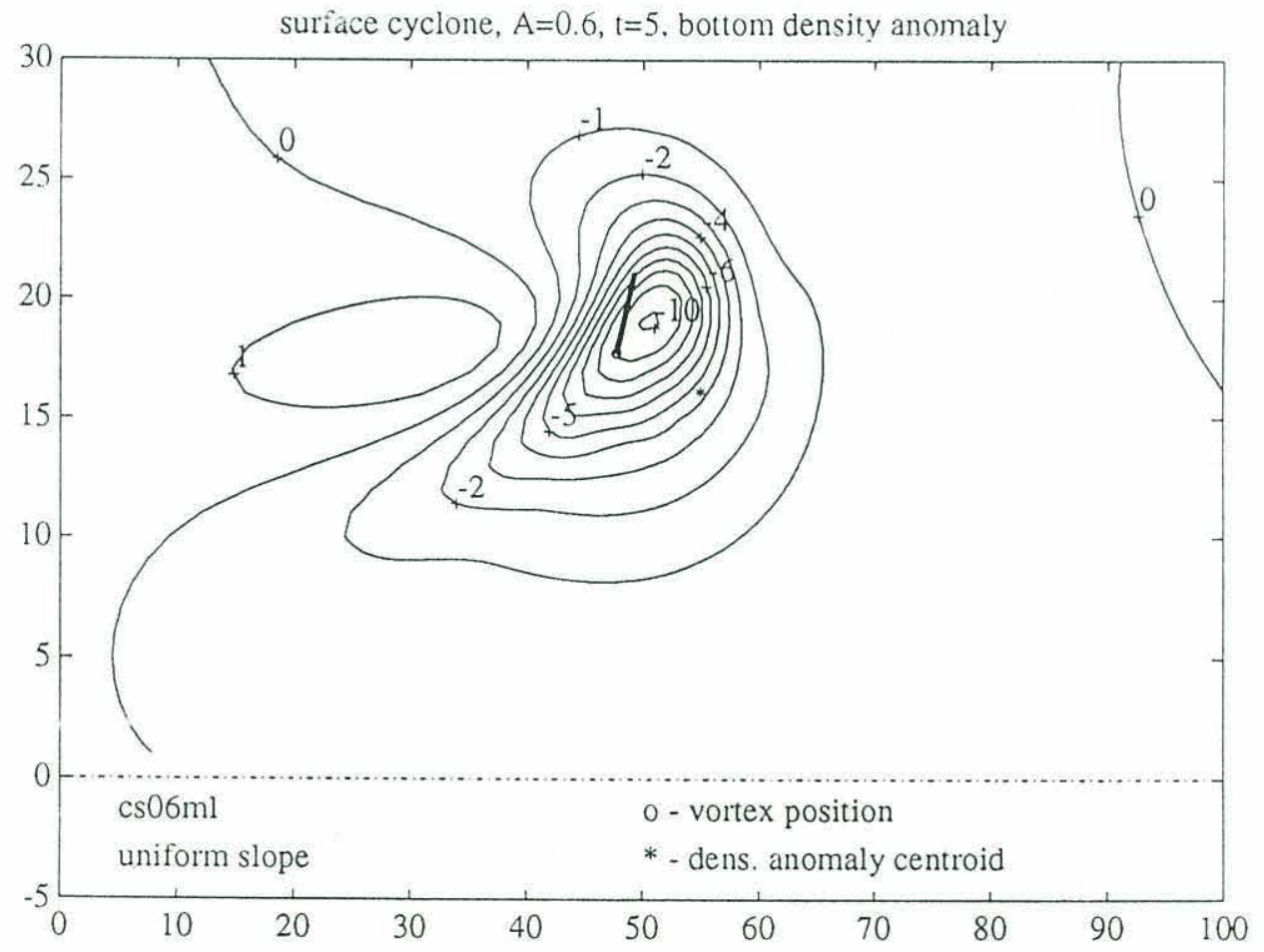

(b)

Figure $5.11(a, b)$. The vortex trajectory (a) and a bottom density anomaly (b) for a cyclonic vortex with $\widehat{A}=0.6$ and bottom boundary condition at the actual depth (as in other experiments). 
surface cyclone, $\mathrm{Av}=0.6, \mathrm{tf}=5$

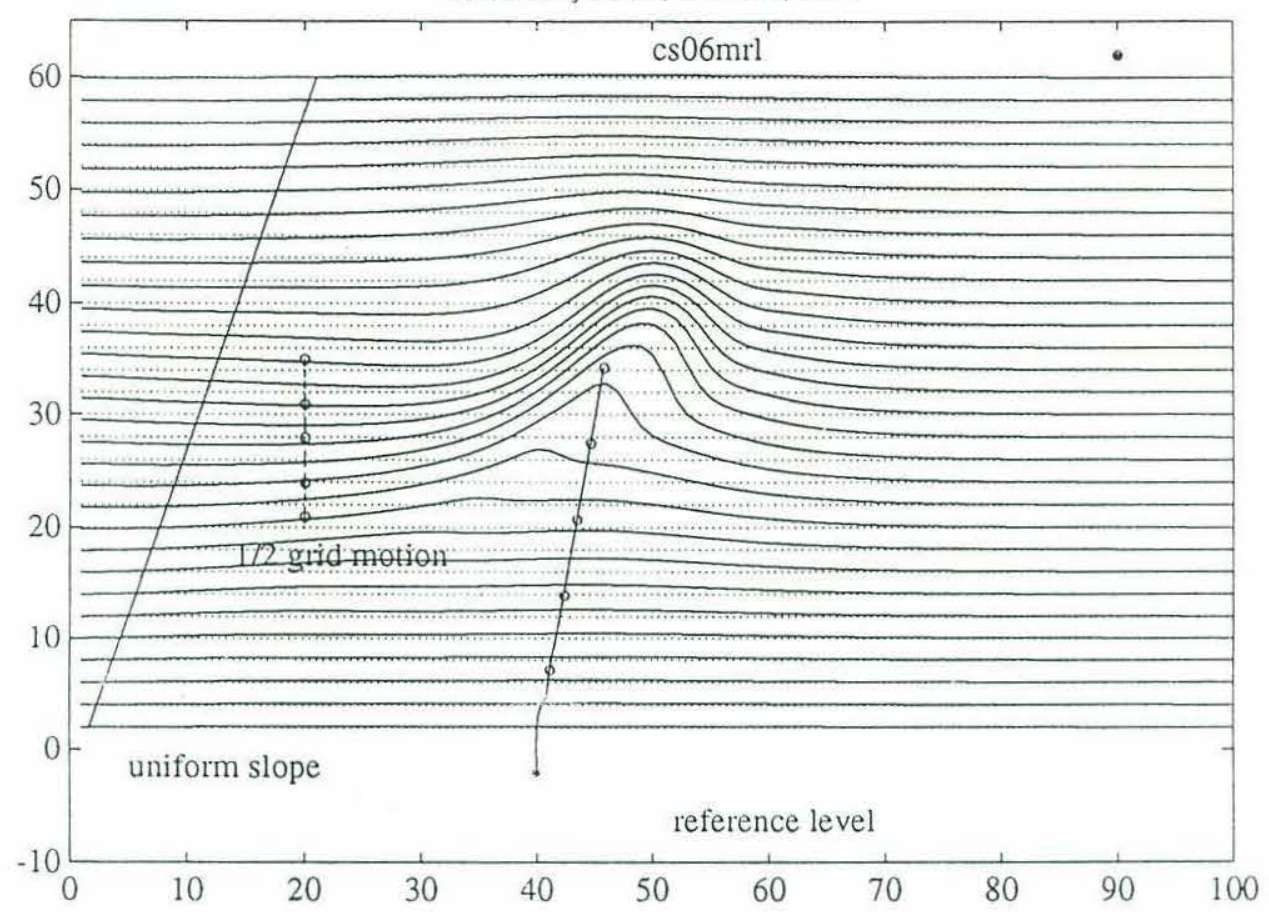

(a)

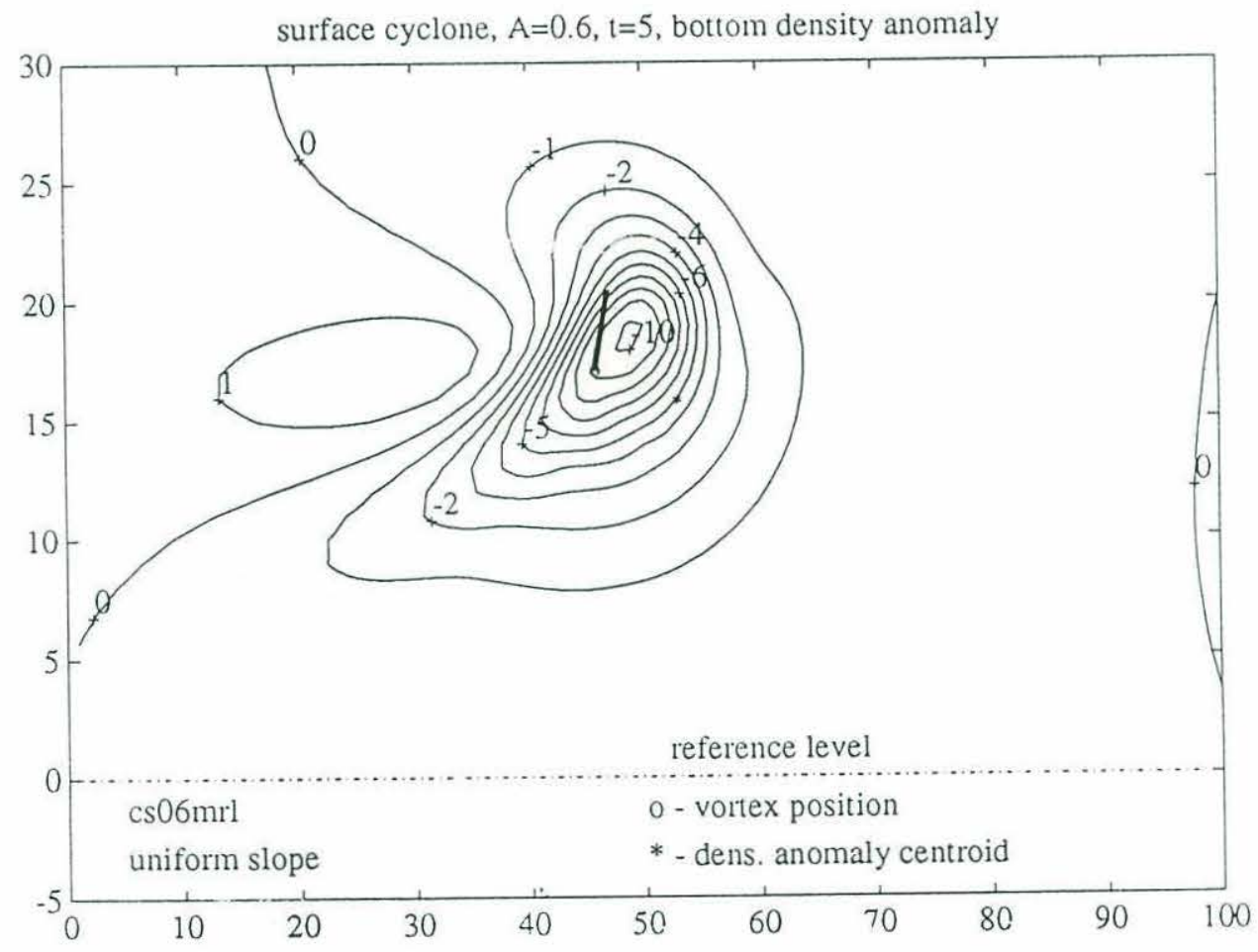

(b)

Figure $5.12(\mathrm{a}, \mathrm{b})$. Same as for $5.11(\mathrm{a}, \mathrm{b})$ but the bottom boundary condition at the reference depth 
surface cyclone trajectory, $A=0.1, t f=15$

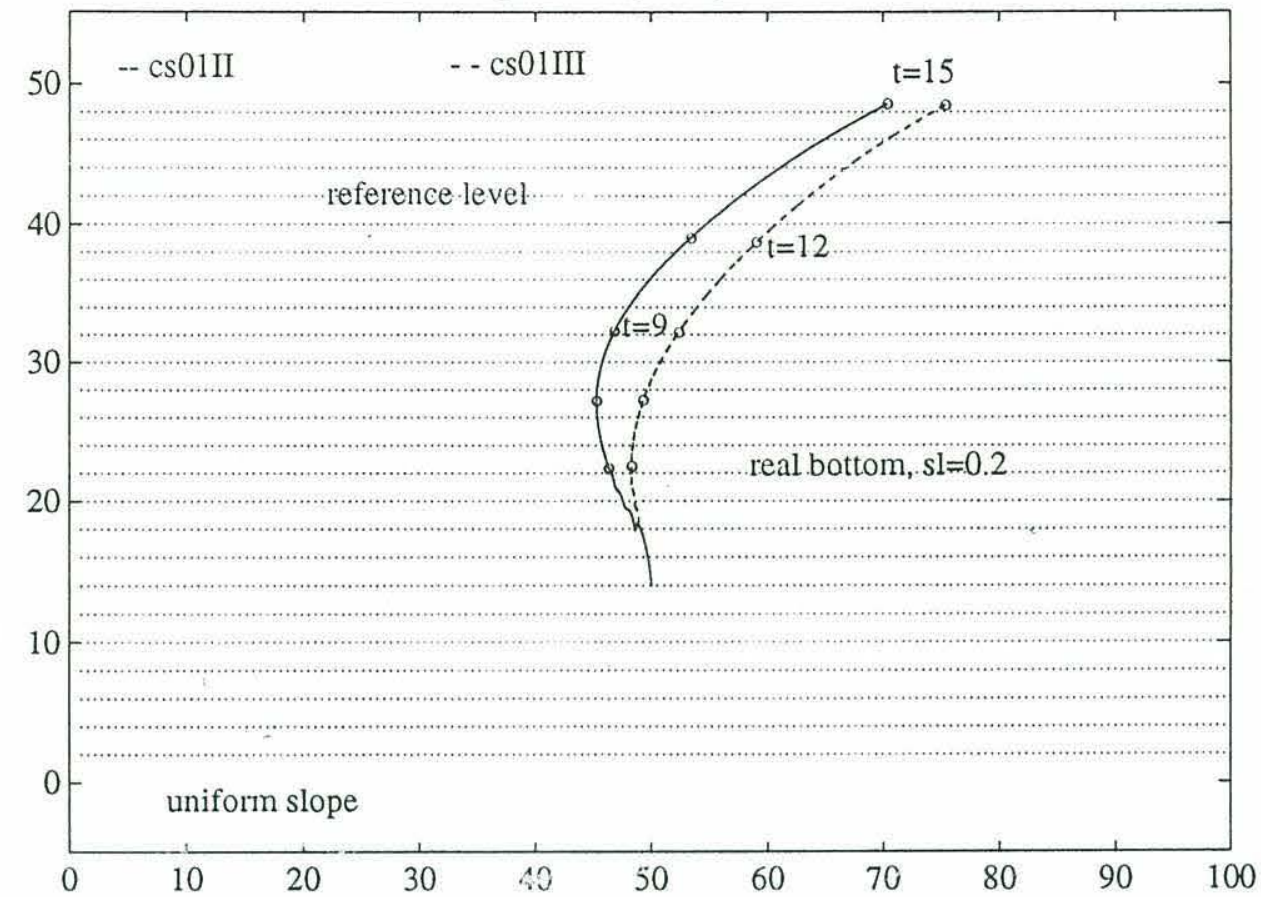

Figure 5.13. Trajectories of identical-twin experiments with a fixed grid with bottom boundary condition at the reference level (solid line) and the actual depth (dashed line).

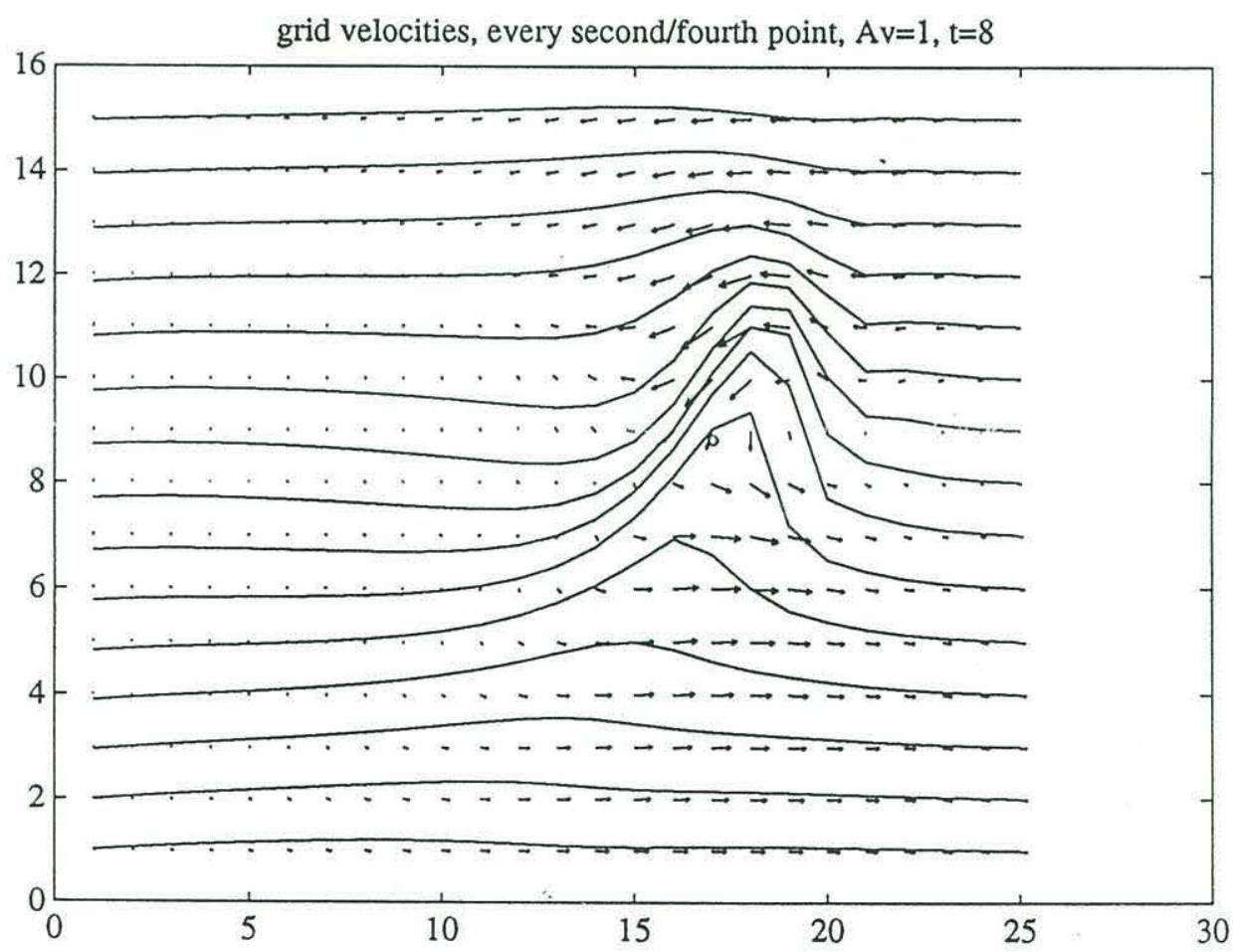

Figure 5.14. Velocity field at the bottom for a steadily translating state. 
Labeling of these runs contains some information about the sense of rotation and the strength of a vortex, type of the grid, and also in some cases necessary information about topography and a background flow. For example, the first plot (figure 5.8) shows the results of the experiment "cs04ml"; here first letter "c" indicates a cyclonic vortex (versus "a" for anticyclones), next letter "s" means a surface vortex, number "04" shows its strength $\widehat{A}=0.4$, then " $m$ " - for the moving grid and the last "l" stands for a "large grid" (some earlier experiments were performed in a smaller domain).

Now consider these pictures in some detail. Generally cyclonic vortices move in the nearly "northward" direction with some "eastward" component of velocity (to the right in these pictures). At certain moments (for example at $\mathrm{t}=.5$ in figure 5.7) the "eastward" component actually dominates, but then motion again becomes predominantly "northward". Stronger vortices move faster. Moreover, their speeds typically increase faster than the first power of the amplitude $\widehat{A}$. An "eastward" component of trajectories also increases faster with the amplitude than the "northward" one, which leads to a larger angle between the trajectory and the northward direction for stronger vortices. It happens because a density anomaly trails further behind with increasing strength. This angle is approximately $10^{\circ}$ for relatively weak vortices $(\widehat{\mathrm{A}} \sim 0.2 \div 0.4)$ and increases up to about $20^{\circ}$ for strong vortices $(\widehat{\mathrm{A}} \sim 2$ ). But even strong vortices over relatively smooth slopes move in the predominantly cross-slope direction. It should be mentioned also that when the surface is absent the vortex trajectory is inclined slightly "westward" instead of the "eastward" direction, although the difference in velocities with and without the surface is quite small. We consider this in some more detail in chapter 6.

The density anomaly has a bean-shaped structure with a maximum on the left side of the vortex and slightly before it in the direction of motion (upward for cyclones), but its centroid is trailing behind the vortex as we already mentioned above.

It is interesting to note that even very strong vortices (relative to the slope angle) in our calculations do not cause breaking of topographic Rossby waves, although they can make density contours strongly shifted and curved from their unperturbed positions. Two factors can contribute to this failure of wave-breaking: first - the velocity field at the bottom is very smooth and perturbations are spread over an area of several deformation radii around a vortex; and second - strong vortices move very fast and before waves succeed to break a vortex moves away from the area of a maximum current perturbation.

When the grid is fixed the behavior of vortices is very similar to the moving grid case when the vortices are far from the boundaries, but as they move closer to the edges their trajectories become more curved and tilted toward the along-slope direction because of the cross-slope asymmetry. Completely steady translation is not achieved in this case and as the vortex moves out of the domain its translation becomes almost along-slope, although the solution is not accurate in this region. 
In these experiments with a simple uniform slope we wanted also to examine the influence of referencing the bottom boundary condition. In most experiments we put the bottom boundary condition (evolution of density function $\eta$ ) at the actual depth, that is, at different levels for upper and lower regions in the top view of the domain. But we also performed some identical-twin runs where we put this boundary condition at the reference depth of the middle of the domain. Figures

5.11 and 5.12 show trajectories of the vortex of the amplitude $\widehat{A}=0.6$ with the actual and the reference depth for the bottom boundary condition. These trajectories look very similar, except that the one with a reference depth is more "vertical", that is its "eastward" velocity component is slightly smaller than for the actual depth case. The cross-slope "northward" velocity is almost identical for two cases. This can be explained from the point of view that putting the boundary condition at the actual depth will introduce some cross-slope asymmetry into the solution (particularly in the pressure field around a vortex). This cross-slope asymmetry leads to some along-slope component of vortex motion because of geostrophic dynamics.

Another pair of identical-twin trajectories (from a different set of experiments) is shown in figure 5.13 for the runs with the fixed (unmoving) grid. It shows that the trajectories are more tilted from "northward" direction because of the edge effects, but the difference between the actual and the reference depth experiments is similar: the reference depth trajectory is slightly more "vertical" than the one with the boundary condition at the actual depth.

Figure 5.14 shows the approximate velocity field at the bottom for the steadily translating state of a moderately strong cyclonic vortex with $\widehat{\mathrm{A}}=1$. It indicates a rather complicated spread structure resulting from a combination of the opposite-signed vorticity fields from the bottom density anomaly and a vortex itself. Near the center (where the vortex is situated) a cyclonic circulation is prevalent and at the upper and lower edges of the domain there is an anticyclonic shear, with velocities almost parallel to the slope. Further from the vortex the velocity decays rapidly because these cyclonic and anticyclonic circulations compensate each other. The mechanism of this compensation and of the vortex propagation will be the subject of the next two sections of this chapter.

\section{6 "Hetonic" translation mechanism}

As we already mentioned above, a vortex is advected by a bottom density anomaly which has approximately the same opposite-signed total circulation as a vortex itself. If this anomaly is symmetrical and centered just under the vortex it would not move at all. But this opposite-signed 


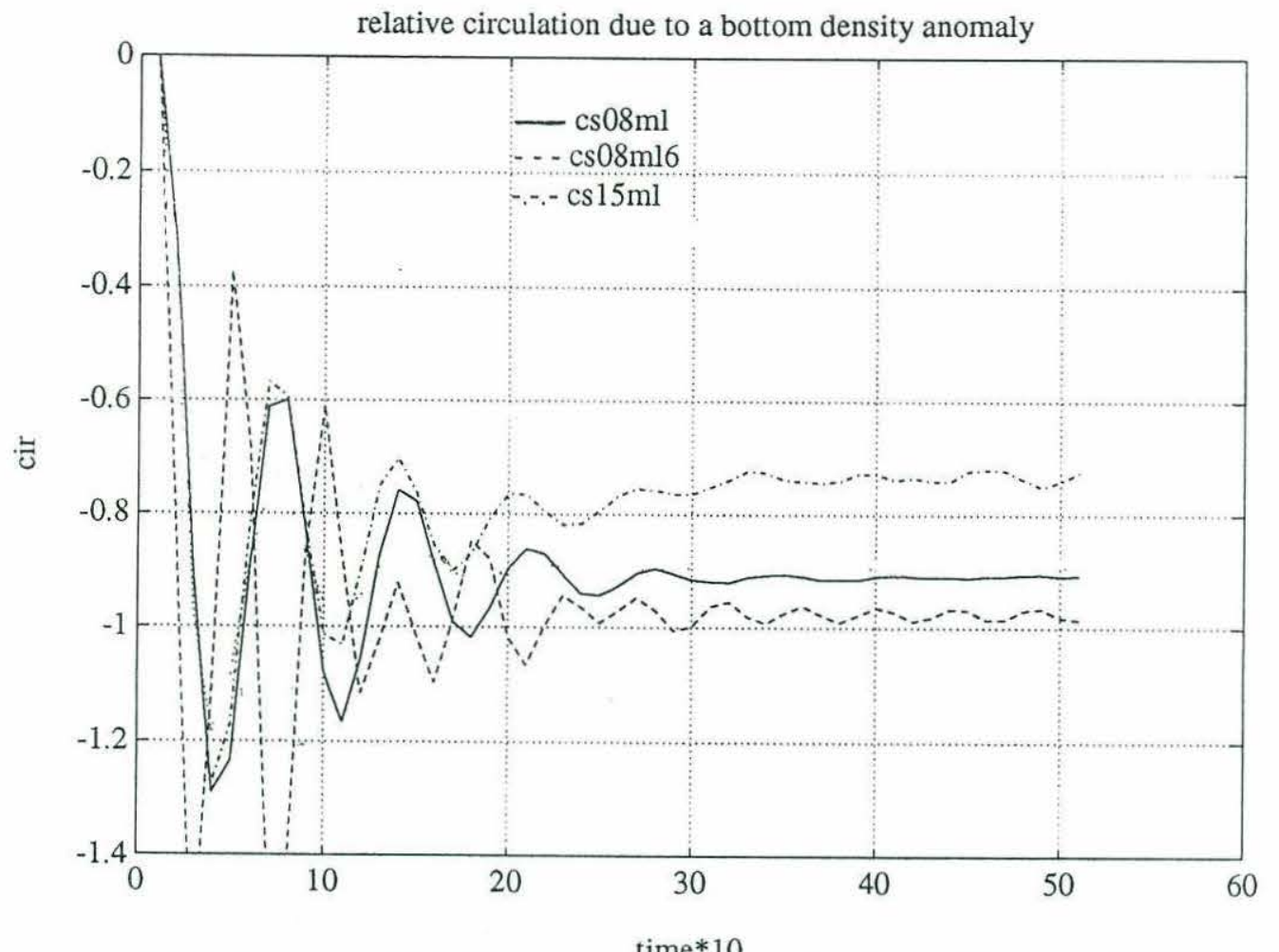

Figure 5.15. Evolution of the total circulation from the bottom density anomalies, normalized by the vortex circulation, for several runs.

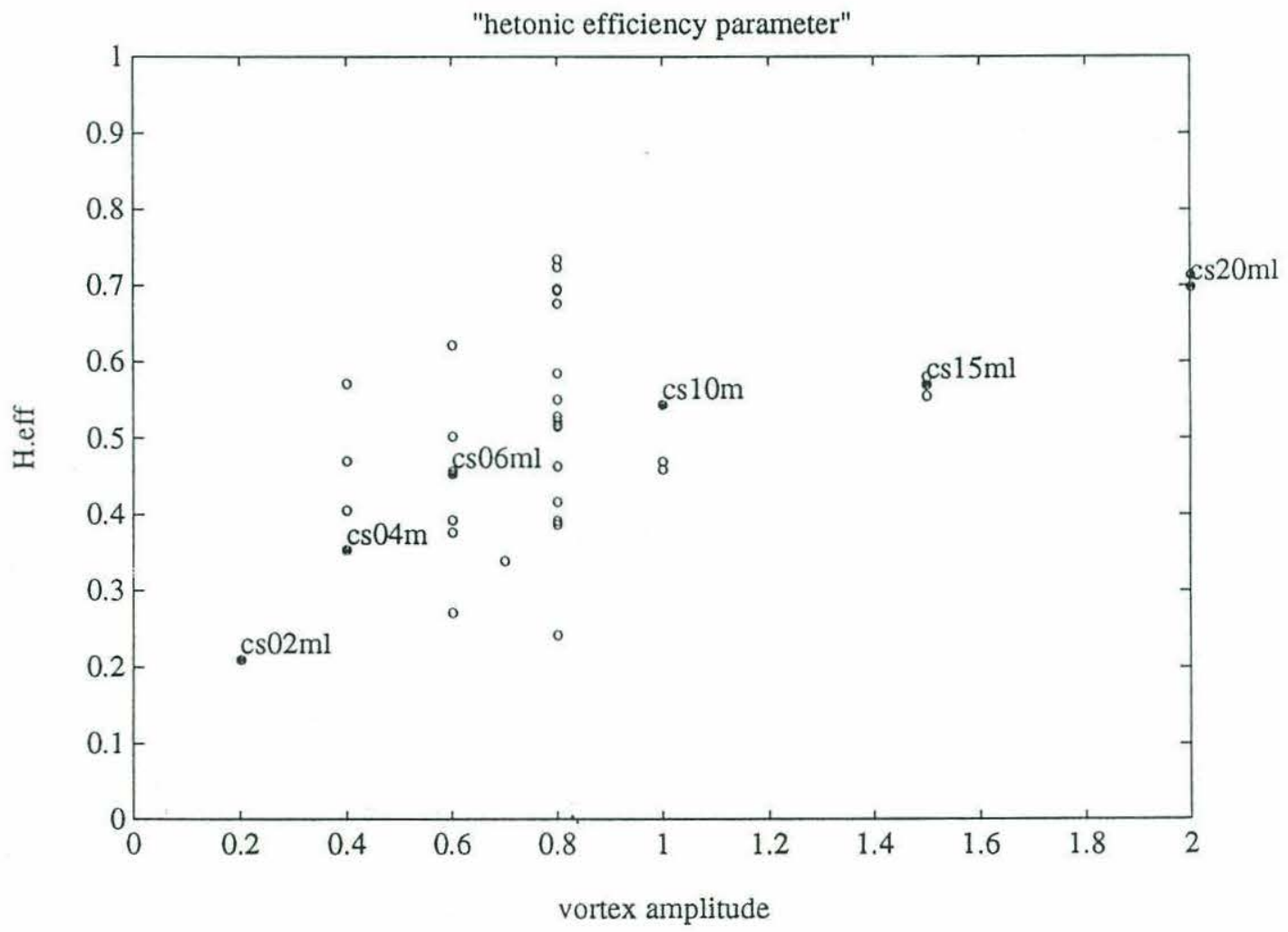

Figure 5.16. "Hetonic efficiency parameter" versus amplitude $\widehat{A}$ for different experiments 
anomaly is shifted horizontally from the vortex position and resulting structure resembles a vertically tilted dipole. Such a dipolar structure was studied before in a different context because of its interesting properties. A general problem of motion of several point vortices at different depths (or in different layers) was considered by Gryanik (1983) and more specific example of two oppositesigned vortices in different layers - by Hogg and Stommel (1985), who coined the term "heton" for such a dipolar structure because it is capable of transporting heat. Steady translation of a dipole is possible only when the upper-layer and lower-layer vortices are opposite-signed and of the same strength, otherwise the pair will move in circles. A heton represents an elegant solution and was used in several studies concerning vortex dynamics and baroclinic instability (for example, Pedlosky, 1985). Yet it is not clear how often such structures can occur in the real ocean. First of all, the requirement that strength of the upper-layer vortex should match that of the lower one is rather stringent to be satisfied often. Another problem with the realistic case is that once such dipole is formed, vertical shear which is usually present in the large-scale ambient flows or induced by other vortices can easily stretch it apart. If vortices are significantly separated vertically they are not very strongly coupled to overcome shearing. Such interaction of vortices with a vertical shear flow was studied in several works, for example Walsh (1992), who developed a multi-layer contour dynamics model for such problem, Yano \& Flierl (1992), Meacham et al (1993).

It is interesting that in our case such a hetonic structure is a natural outcome of the vortextopography interaction. An opposite-signed vorticity anomaly at the bottom is always shifted horizontally from the vortex position because of the asymmetry of the wave-radiation process. It is created by the vortex itself and does not depend on some external processes so it always stays with the vortex regardless of the ambient shear flows. Its circulation is approximately the same as that of the vortex itself. This density (and vorticity) anomaly is distributed over a large area with a horizontal scale more than the "stretched" depth (several deformation radii). But its total circulation nearly compensates for that of the vortex. Below we shall present some theoretical considerations why there should be an exact compensation for steady motion. So the whole system, a vortex and a density anomaly at the bottom, indeed represents a hetonic structure.

To check this suggestion about the "hetonic mechanism" of vortex motion we computed evolution of total circulation due to the bottom density anomaly in our numerical experiments. Some examples are shown in figure 5.15. One can see a strong initial "overshoot" of the bottom vorticity because of such unbalanced initial conditions, so after about one half of the short waves period the bottom circulation exceeds the vortex strength by almost two times. Then after some rapidly decaying oscillations it remains almost constant with a values between -.75 and nearly -1 (one unit is the vortex strength $\widehat{A}$ ). These oscillations are somewhat stronger than those of the vortex trajectory which reflects some processes at smaller scales in the bottom vorticity field. The vortex motion on the other hand depends on the gross, integral structure of the density anomaly and 
naturally it reaches steady regime much faster. Persistent oscillations of the bottom density anomaly are also due to the moving grid technique which also introduces a small amount of discontinuity and unsteadiness to the process.

The actual computed circulation at the bottom is less than the vortex strength. It can be attributed to the fact that our domain is finite and the density anomaly is highly delocalized so it is truncated at the edges of the domain. The evidence of this fact is that results are most close to unity for the run "cs $08 \mathrm{ml6}$ " which is performed with the depth of the model equal to 6 units instead of standard 10 units in other runs, so the effective horizontal lengthscale of the domain was larger in this run which means less truncation error. Also our smoothing and attenuation procedures could contribute to this minor discrepancy. As the strength of the vortex increases the bottom vorticity field gets more delocalized and so the difference between the bottom circulation and the vortex strength increases. For example for $\widehat{\mathrm{A}}=1.5$ (run "cs $15 \mathrm{ml}$ ") the circulation at the bottom reaches up to only approximately 0.75 of the vortex strength.

Nevertheless, the tendency of the bottom vorticity field to compensate for the vortex circulation and form a baroclinic heton-like structure is evident in all our experiments. This allows us to introduce a nondimensional parameter $v$ characterizing the translational velocity of the vortex relative to the maximum velocity which can be achieved by a "heton" structure. I described already this parameter $v$ in the section 5.2. It can naturally be called the "hetonic efficiency parameter" because it reaches unity when translational velocity is equal to that of the "optimal heton" (see the section 5.2 for discussion of this). I computed this parameter for steady translations in our numerical experiments and its values depending on the vortex amplitude $\widehat{A}$ are shown in figure 5.16. The shaded circles correspond to the simple slope cases, without the slope curvature and background flows and the labels of these runs are plotted in this figure. The empty circles correspond to other experiments, with the slope curvature and mean advection. Naturally this parameter is less than unity in all our experiments, but in some cases it is not very far from 1 , its maximum possible value. This shows that this hetonic mechanism of vortex translation over a topographic slope is rather "efficient". Interestingly enough, the efficiency of a "hetonic mechanism" increases with the amplitude from about 0.2 for very weak vortices to approximately 0.75 for strong ones $(\widehat{\mathrm{A}}=2)$. Large scatter in this figure is due to the fact that this plot shows experiments under very different conditions: with and without slope curvature, background flows, etc.

Below in the next section we shall try to explain this "hetonic" tendency and shall formulate a general statement when and why this compensation should occur and its implications for interaction of vortices with topography. 


\section{6 "No net barotropic vorticity" theorem}

In the previous section we showed a propensity of a vortex over a topographic slope to form a density anomaly at the bottom with a circulation compensating that of the vortex itself. The whole structure therefore tends to be purely baroclinic, with a zero total circulation. Since this tendency persists throughout practically all of our experiments there should likely be an explanation why this phenomenon is so ubiquitous.

This tendency strongly recalls conditions of the "zero angular momentum theorem" of Flierl, Stern and Whitehead (1983) concerning motion of an isolated vortex on a planetary beta-plane. In a simple form their theorem states that there can be no isolated slowly-varying monopolar vortex on the planetary beta-plane (with a net circulation and, hence, angular momentum). If such a vortex perturbation exists initially it should start developing a secondary field around itself to compensate for the net initial circulation. This theorem has a dynamical sense - it is proven by integrating the (primitive) equation for a rotating fluid on a beta-plane over the whole infinite domain. It requires also no possibility of angular momentum input through the boundaries - and therefore it is restricted to the flat surface and bottom case. We study the situation with a sloping bottom and the "zero angular momentum theorem" is not applicable here in its initial form. Flierl (1987) actually discussed the conditions of steady translation for a case with a sloping bottom and inferred that the integrated pressure field at the bottom must be zero, because this would imply no net input of angular momentum through the boundaries. But we shall present our statement in the kinematical, rather than the dynamical form, without mentioning a pressure field. In our case the dynamics has much in common with the planetary beta-plane and such property of developing a compensating circulation is also evident.

In this section we present a statement about the zero-circulation property of isolated eddy-like perturbation in geophysical fluids. It is different however from the above mentioned theorem. It is mainly of kinematical rather than dynamical nature and its proof does not depend on specific equations of motion but rather on the geometrical pattern of a flow. Our statement is neither a generalization nor a particular case of the "zero angular momentum theorem" although in certain cases both theorems are applicable.

We would like to present our statement in a general, even somewhat abstract form, to disconnect it from the specifics of our case (a vortex over a slope) which was its initial motivation and to underline its kinematical nature. The theorem itself is written below. 


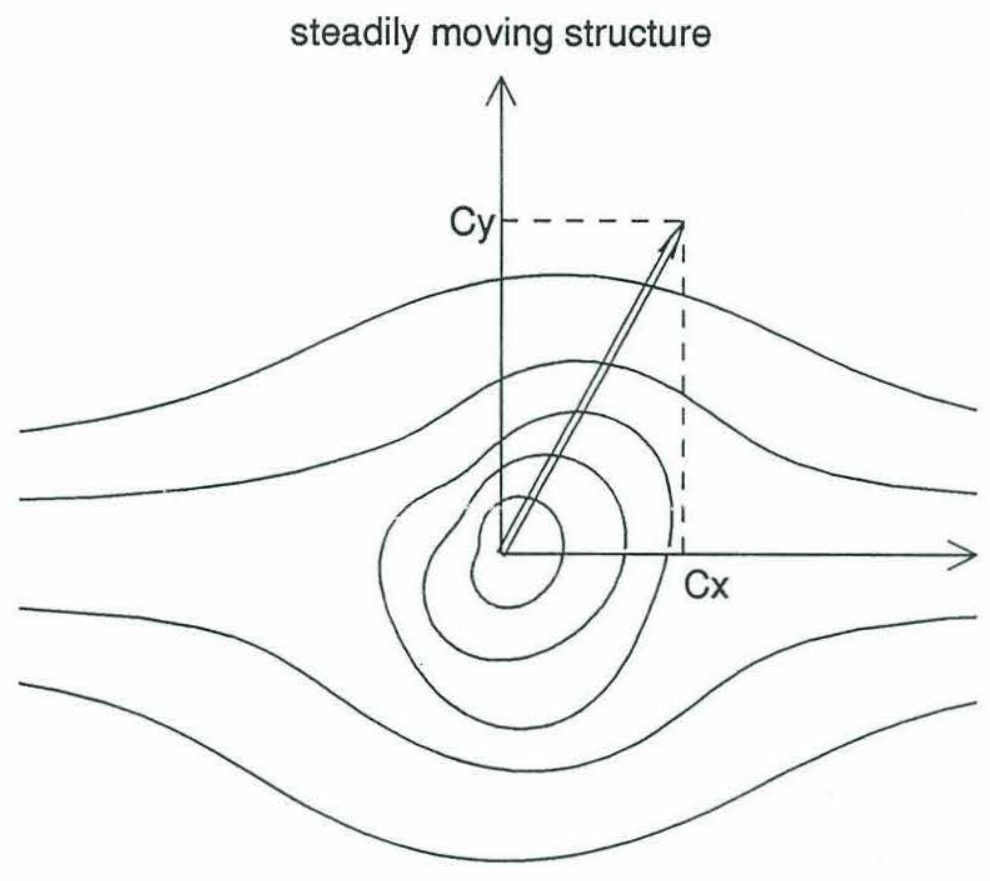

(a)

displacement of tracer isolines

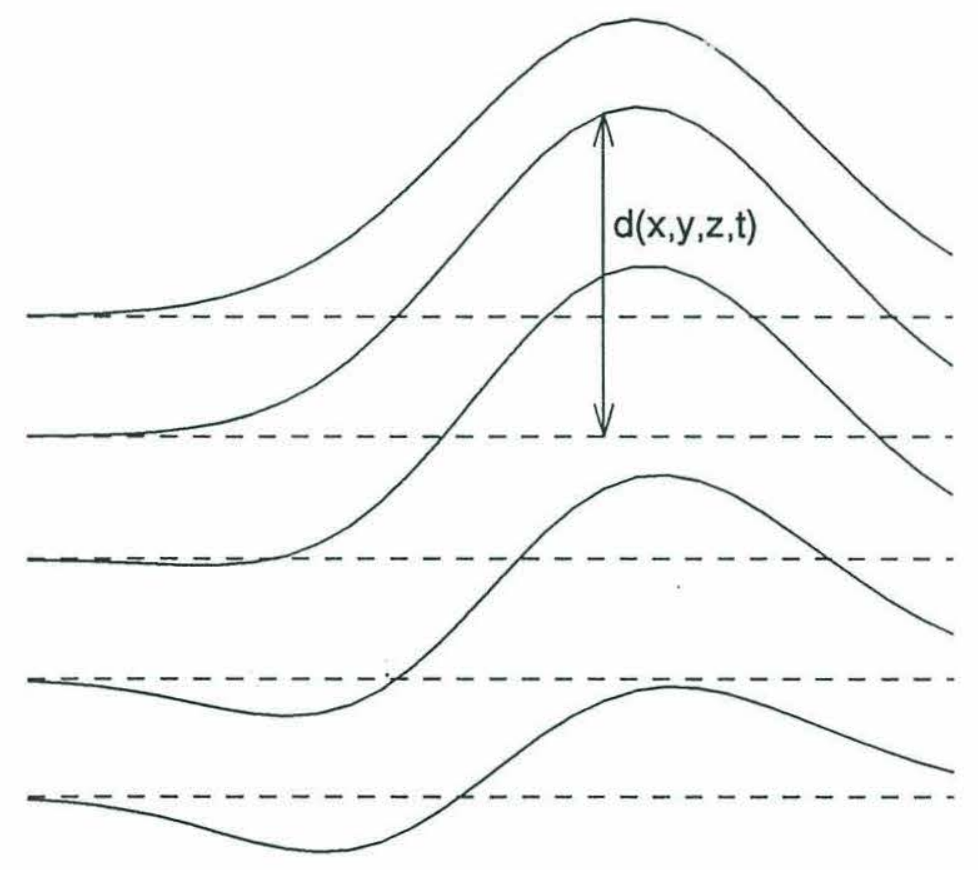

(b)

Figure 5.17 (a,b) (a) Sketch of a steadily moving perturbation.

(b) Displacement of tracer isolines. 


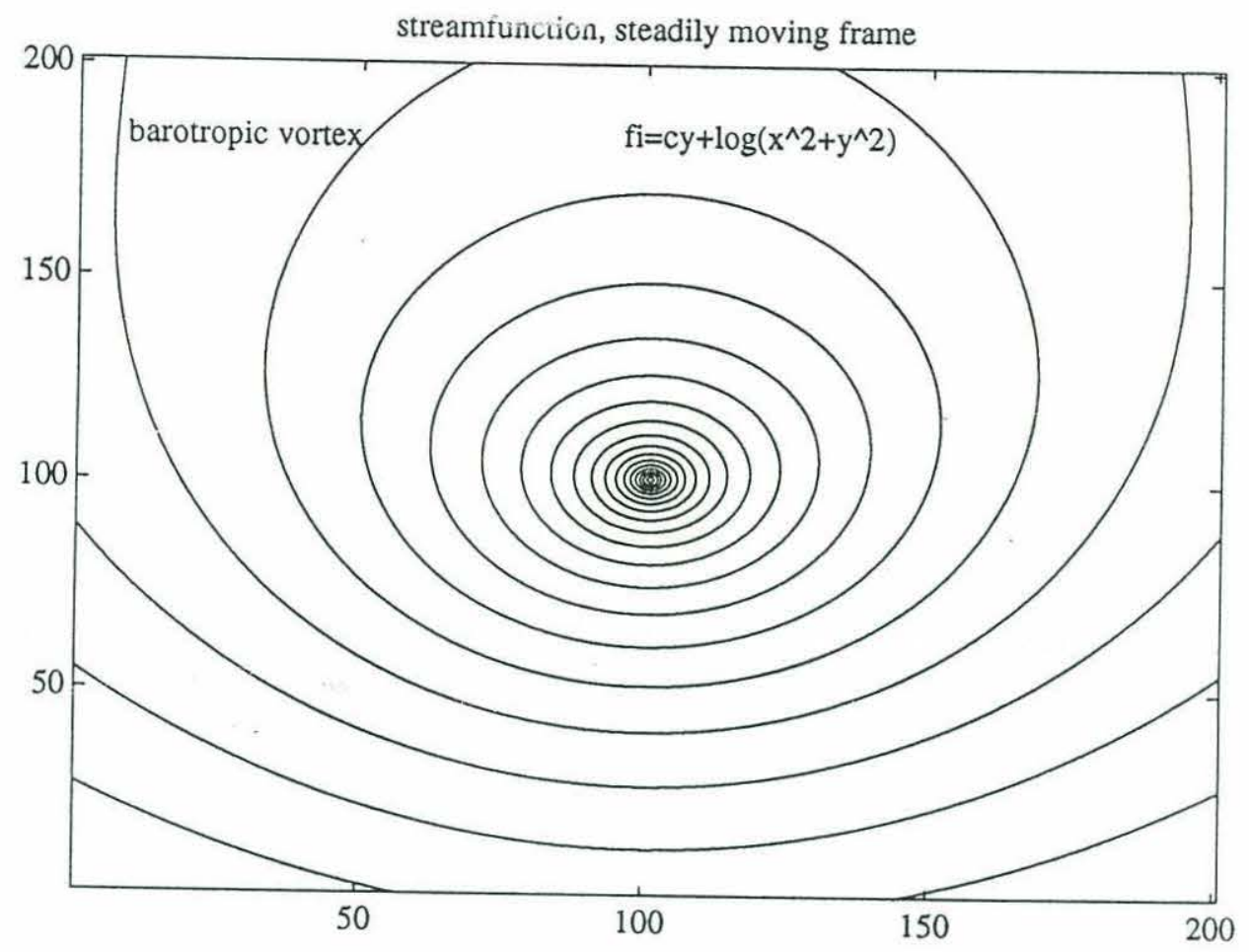

(a)

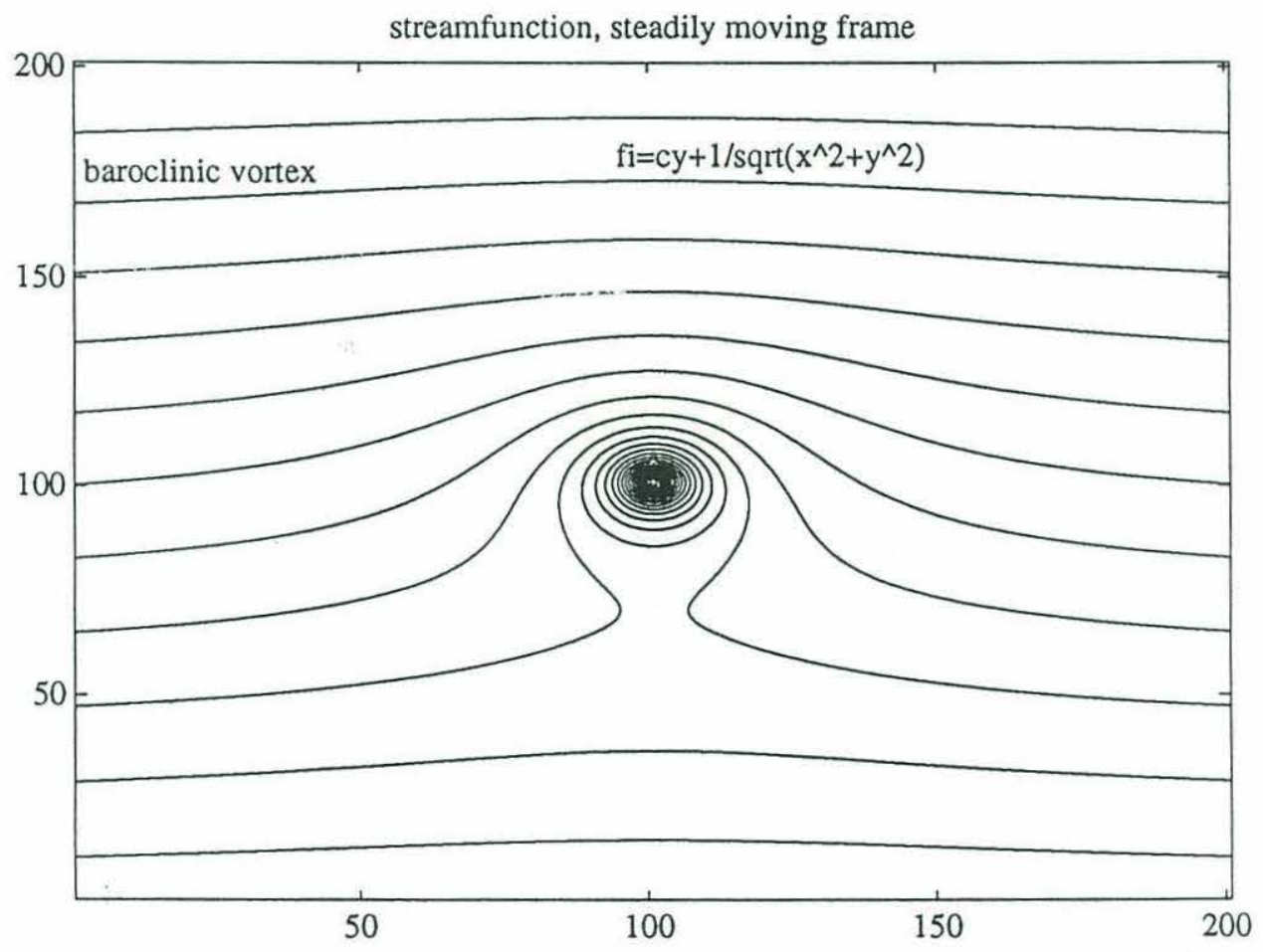

(b)

Figure $5.18(a, b)$. Streamfunction from a perturbation with a non-zero (a) and zero (b) total barotropic component. 
The "no net barotropic vorticity" theorem:

If a certain structure (perturbation) moves (horizontally) through a continuous medium under the following conditions:

C1. Horizontal velocities are non-divergent:

$$
\mathrm{u}_{\mathrm{x}}+\mathrm{v}_{\mathrm{y}}=0
$$

Hence we can introduce streamfunction $\psi(\mathrm{x}, \mathrm{y}, \mathrm{z}, \mathrm{t})$ :

$$
\mathrm{u}=-\psi_{\mathrm{y}}, \quad \mathrm{v}=\psi_{\mathrm{x}}
$$

C2. Motion is steady and translationally invariant (see figure 5.17a):

$$
\psi(\mathrm{x}, \mathrm{y}, \mathrm{z}, \mathrm{t})=\psi\left(\mathrm{x}-\mathrm{c}_{\mathrm{x}} \mathrm{t}, \mathrm{y}-\mathrm{c}_{\mathrm{y}} \mathrm{t}, \mathrm{z}\right)
$$

C3. Continuous medium has a lagrangially conserved property (tracer) dependent on only one of the horizontal coordinates (y) in the background:

$$
\begin{aligned}
& \sigma=\sigma(\mathrm{y}, \mathrm{z}) \quad @ \quad \mathrm{r}^{2} \rightarrow \infty \\
& \text { and } \sigma_{\mathrm{t}}+\mathrm{u} \sigma_{\mathrm{x}}+\mathrm{v} \sigma_{\mathrm{y}}=0
\end{aligned}
$$

C4. Structure is weakly localized (isolated) in the sense of the two requirements:

a. Velocities decay at infinity

$$
|\mathbf{v}| \rightarrow 0 \quad @ \mathrm{r}^{2} \rightarrow \infty
$$

b. Displacement of particles across a tracer gradient far from the perturbation remains finite at all times (figure 5.17b):

$$
|\mathrm{d}(\mathrm{x}, \mathrm{y}, \mathrm{z}, \mathrm{t})|<\infty \quad \text { @ } \forall \mathrm{t}, \mathrm{r}^{2} \rightarrow \infty
$$

C5. Structure moves at a non-zero angle to the $\mathrm{x}$-axis (isolines of the tracer field):

$$
c_{y} \neq 0 .
$$

Then velocities must decay faster than $1 / \mathrm{r}$ (perturbation must be strongly localized):

$$
|\mathbf{v}(\mathbf{r})| \cdot|\mathbf{r}| \rightarrow 0 \quad \text { @ } \mathrm{r}^{2} \rightarrow \infty
$$

For example if a streamfunction $\psi$ can be expanded into Laurent series: 


$$
\psi=\mathrm{A}_{0} \log (\mathrm{r})+\mathrm{A}_{1} \frac{1}{\mathrm{r}}+\ldots
$$

then the first expansion coefficient must vanish: $\mathrm{A}_{0}=0$.

In the case of a geophysical flow this means the absence of a net barotropic vorticity.

This statement is actually easier to prove than to formulate. One need only to consider the streamlines structure from a perturbation with and without a barotropic component. In figure 5.18 $(a, b)$ these streamlines are shown in coordinate system moving with the perturbation, that is the function $\psi_{\mathrm{m}}=\psi+\mathrm{c}_{\mathrm{x}} \mathrm{y}-\mathrm{c}_{\mathrm{y}} \mathrm{x}$. For the case with a non-zero total barotropic component the streamfunction has a logarithmic term and all the streamlines are closed contours around the perturbation. Because this pattern is stationary in a moving frame (condition $\mathrm{C} 2$ ) this means that the particles are advected along closed contours in this moving frame, no matter how far from the perturbation they are situated. They move with velocities averaged over a period of rotation $\left(c_{\mathrm{x}}\right.$, $c_{y}$ ), and if $c_{y} \neq 0$ (condition C5) their displacement across the tracer isolines is unbounded, which contradicts the assumption $\mathrm{C} 4 \mathrm{~b}$. Therefore the net barotropic term, proportional to $\mathrm{A}_{0}$ must be zero.

On the other hand if only baroclinic component is present (figure 5.18b) there is only a small region of closed streamlines in the moving frame and all the streamlines in the background, far from the perturbation, are open. This means that distant particles do not feel the perturbation strongly enough and their displacements along the tracer gradient will not be infinite. When a singular perturbation and a tracer are separated vertically, there can be a situation without any closed contours at the tracer level, so no fluid with tracer will be trapped. This is what actually happens in our case: there are no closed streamlines at the bottom and deep water is not trapped by a vortex.

The only case when a steadily moving perturbation can have a net barotropic component is when its velocity is directed along the tracer isolines (in our case along the isobaths). Then infinite displacement of fluid particles will occur along these lines, which is permissible by $\mathrm{C} 4$. It is worth noting that our previous self-analytical solution in chapter 3 therefore does not contradict this theorem.

This statement for QG approximation can be proven also by a method similar to proof of the "zero angular momentum" theorem, that is integrating the equations of motion over the whole domain (Pedlosky, personal communication). But I wanted to present a more kinematic explanation, using the streamline pattern rather than the equations of motion.

In the case of vortices in geophysical flows the absence of a barotropic component is in fact quite natural. If solution is to be steady (translating) the vortex must be advected by another vortexlike perturbation, so the system should have a dipolar structure. Only when the perturbation is of the 
same strength and opposite sign there will be a straight-line motion, otherwise the trajectory will be circular.

Another, more tentative explanation as to why there is no total barotropic component when a vortex moves across a slope is that the whole system tends to its minimum-energy state. If a net barotropic component is present, the velocity field is "felt" far away from a vortex and even distant surrounding water parcels will move around a vortex which means that their excursion across the slope will be large and so the changes in potential energy of these parcels. To prevent this the bottom density field is adjusted in such a manner as to limit these cross-slope excursions. The only way to achieve this is to reduce the total barotropic component of the system.

Although our statement is not attached to any specific equations of motion (only horizontal non-divergence) it is in some respect weaker (in the presented form) than the "zero angular momentum theorem". In particular it requires steady motion, while the latter operates with a notion of a "slowly varying" dynamics. The most important conclusion from both theorems is that in the presence of an active tracer gradient in the background (potential vorticity or density at the bottom) flow with an initial uncompensated perturbation will evolve toward such a structure which minimizes "mixing" of the tracer, prevents large displacements of fluid parcels across its unperturbed isolines. This is achieved by minimizing the total (integrated over a whole domain) barotropic component of a flow because of its "long-range" influence on the velocity field. Therefore we can expect this tendency to be more general than only under the strict conditions of our theorem and to hold for near-steady motion, not completely isolated perturbation and some $\mathrm{x}$-dependence of the background tracer field (for example for topographic slope with some curvature in our case). Our experiments show this tendency not only for steady motion over a uniform slope but also in the presence of topographic variations and background flows.

Yet there should be limitations to this tendency when the flow properties depart further from the conditions of our statement. We should not expect necessarily the absence of a net barotropic component in the case of very rough topography, presence of strong and unsteady background flows. It would be interesting to examine the limits to which this "no barotropic flow" tendency holds in a realistic case. Consider, for example, the following thought experiment. If we have a flow with a few strong eddies well-separated from each other in the region with a large-scale bottom slope these eddies will be effectively isolated and "independent" because of the lack of the long-range barotropic influence on one another. If we increase the number of eddies (decrease their separation) then at some point "no barotropic flow" tendency may break down and eddies would feel each other much stronger. This suggests that there can be a "threshold" (although not a very distinct one) in eddies population density under which they behave almost independent from each other and above which they represent a strongly interacting turbulent ensemble. 
These considerations can be also relevant to the planetary beta-plane dynamics. Many observations show strong monopolar eddies with a seemingly non-zero barotropic component. However the planetary vorticity gradient is typically not very large in comparison with vorticity of eddies themselves, so the strongest vortices can overcome this "zero angular momentum" tendency, at least locally. Thus we should not expect it to hold in the most energetic regions of the ocean. On the other hand the topographic beta-effect is usually much stronger than the planetary one on typical continental slopes and this tendency is more likely to survive. One can expect that while the influence of the planetary beta-effect is more ubiquitous and present practically everywhere in the ocean, topographic influence, although limited to a small portion of the ocean area, should be stronger where it is present. 


\section{Chapter 6}

\section{Different factors influencing}

\section{vortex-topography interaction}

\subsection{Curvature of the bottom and topographic irregularities}

In previous chapters we presented rather simple and explainable results concerning motion of a point surface vortex above a straight-line topographic slope with a constant angle. This certainly falls short of the realistic treatment of such a problem. In reality the motion of a vortex over a slope is accompanied by many other forces and influences present in the active turbulent ocean with a complicated bottom relief.

In this chapter we are going to examine the influence of various factors on the vortex behavior above a topographic slope. Among these factors one can list non-uniform topography, including large-scale variations and local irregularities, the planetary beta-effect and the presence of steady and unsteady background flows. Actually in this chapter we shall study the influence of only some of these: topographic perturbations, background flows with a vertical shear and also the finite volume of a vortex and non-uniform background stratification.

Uniform slope topography which we considered in the previous chapter is a special case with the topographic forcing symmetrical in some sense around a vortex. By this we mean that in the case of a uniform slope a given cross-slope displacement of density contours leads to the same density anomaly regardless of where this displacement occurs. Because the initial velocity field is circularly symmetric around a vortex it gives rise to a density anomaly which is symmetrical in the cross-slope direction and therefore the along-slope velocity component of a vortex is zero at the beginning. But when the slope angle varies, the same displacement will produce a greater density (and hence vorticity) anomaly at places of a steeper slope and a weaker anomaly where a slope is smaller. Because of the geostrophic dynamics this can lead to stronger along-slope motion of a vortex.

The bottom topography in the real ocean is far from uniform, with large variations of a slope angle and irregularities at all scales. A solution may depart significantly from the uniform slope case 
when various factors are present which introduce the above mentioned asymmetry into dynamics. In this section we want to explore the influence of such topographic asymmetries on vortex motion. First we shall consider cases with straight-line topography, but where a slope angle can vary in the y-direction (cross-slope); we shall refer to this situation as a slope with a curvature. Then we shall give some examples of motion over topographic irregularities (although not very rough ones) where a slope angle can vary in both horizontal directions.

To study the influence of a slope curvature we shall use a bottom elevation in the form of a second-order (quadratic) polynomial of the cross-slope coordinate $y: \tilde{b}(y)=\tilde{\alpha} y+\frac{\gamma}{2} y^{2}$ where $\gamma$ is the approximate curvature of a slope and is assumed to be small.

The above considerations about the increase in the along-slope velocity component of vortex motion are generally confirmed in our numerical experiments. In figure 6.1(a,b) we show the trajectory of the cyclonic vortex over the slope with a positive curvature (the slope angle increases "northward"). While the "northward" velocity component is practically unchanged, the trajectory significantly inclined "westward". This is caused by a certain displacement of an opposite-signed density anomaly upslope. Even if positions of the density contours remain unchanged the resulting density anomaly will be stronger on the "northern" side of the vortex because of the steeper slope angle there. An anticyclonic vorticity field produced by this density anomaly will advect a vortex approximately perpendicular to its centroid and in the clockwise direction, which results in the "westward" velocity component. For an anticyclonic vortex the bottom vorticity field will have the opposite (cyclonic) sign and will advect vortex "eastward", as well as downslope. This mechanism is schematically shown in figure 6.6(a) where we summarize the results for all runs with straight-line topography, for both positive and negative slope curvature. We shall discuss this figure below, after considering several more examples of vortex motion.

When the slope curvature is negative the maximum of the bottom density anomaly is shifted downward, in the direction of increasing steepness. In this case a vortex trajectory is deflected "eastward" for cyclones and "westward" for anticyclones. An example of such motion for the moderately strong cyclonic vortex is shown in figure $6.2(\mathrm{a}, \mathrm{b})$. The motion appears steady while the slope changes, because of the "moving grid" technique. In this case the trajectory is more inclined alongslope than with the same curvature of the positive sign. The bottom density anomaly is more elongated rather than having a bean-like shape as in the cases of a uniform slope. Its centroid is situated farther from the vortex which leads to a more efficient "hetonic mechanism" and a slightly greater translational velocity. For anticyclones the situation is opposite: their trajectories are inclined "westward" over a slope with a negative curvature, and "eastward" - with a positive curvature. "Eastward" translation is slightly faster than westward and a trajectory in this case is more 
surface cyclone, $\mathrm{Av}=0.6, \mathrm{t} f=5$

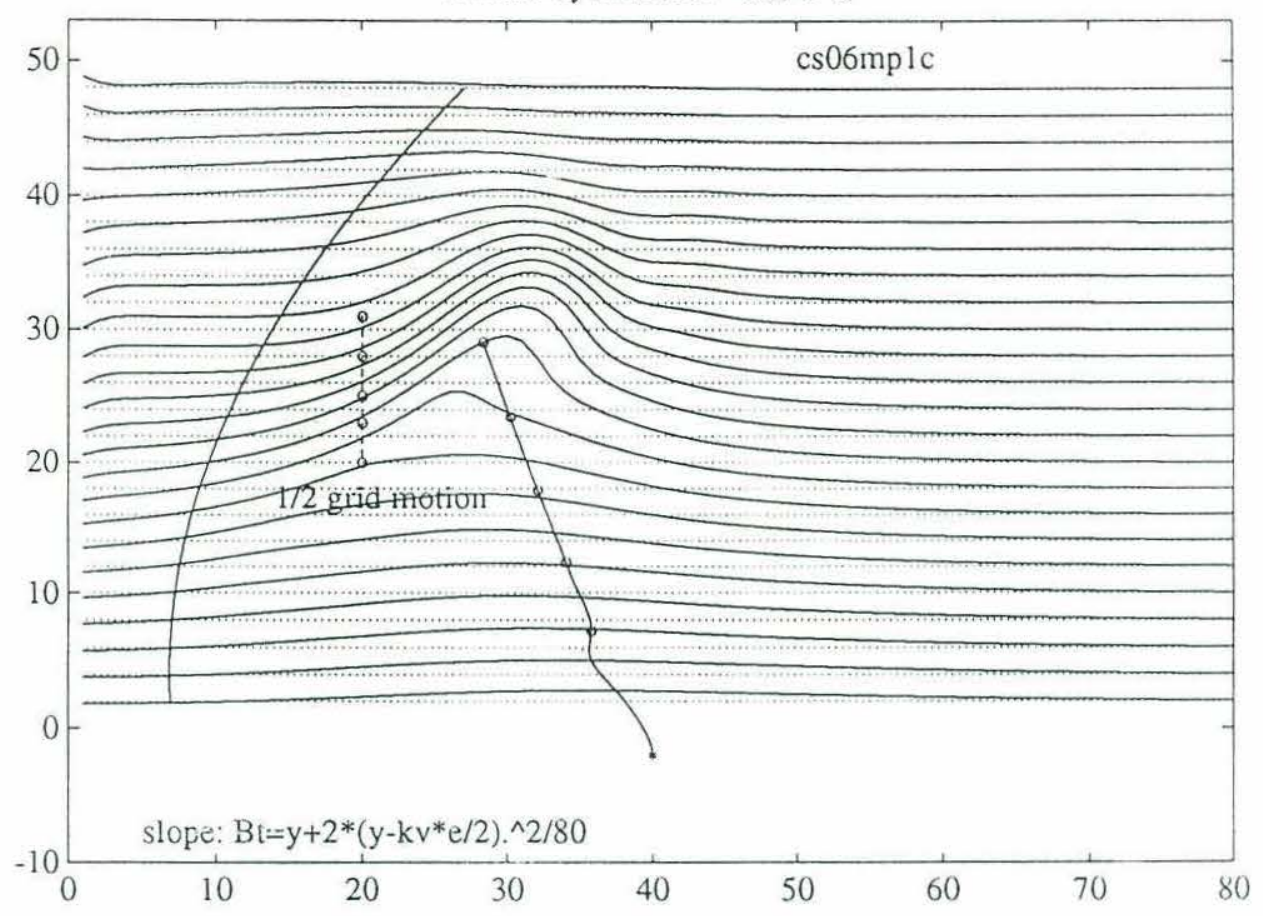

(a)

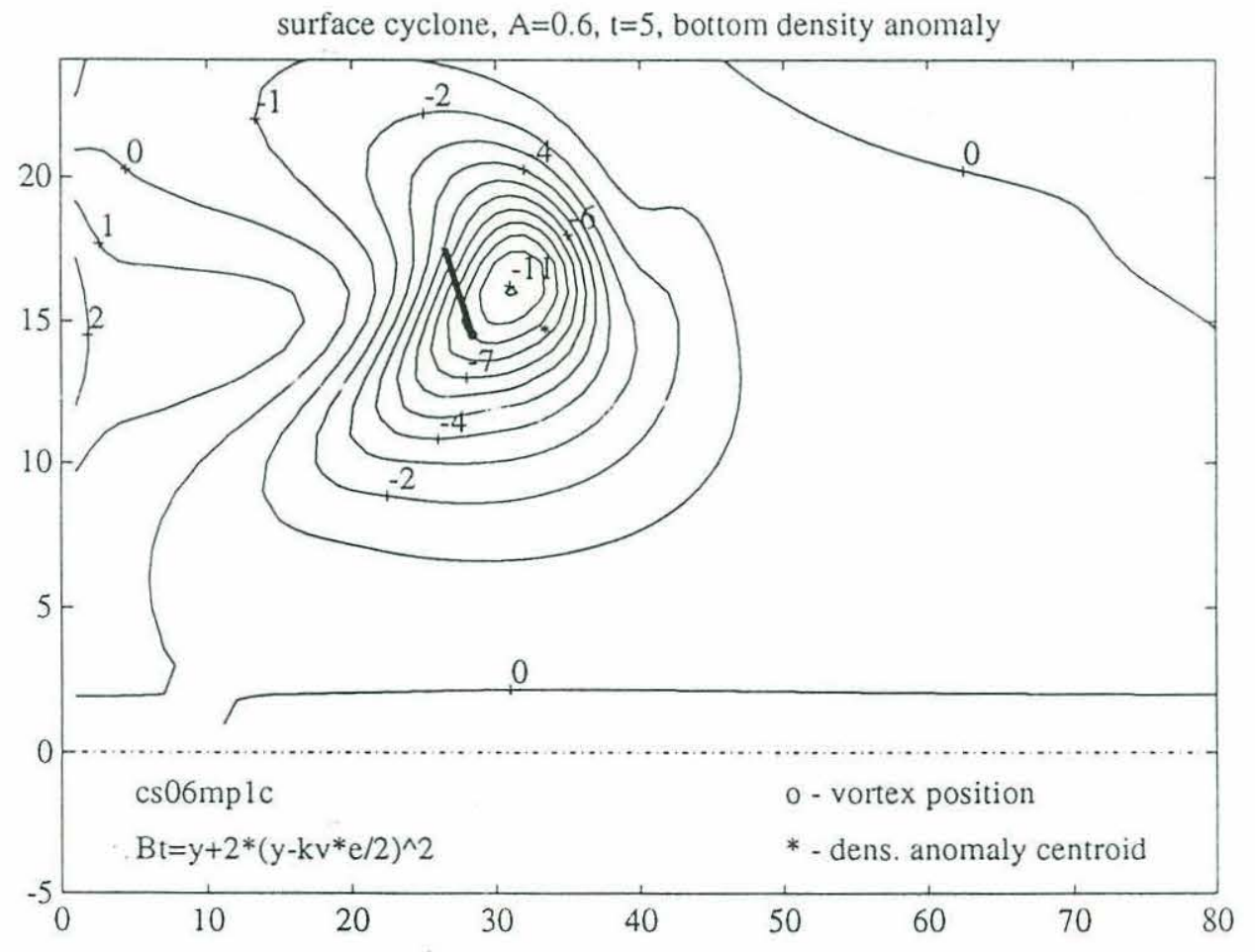

(b)

Figure $6.1(\mathrm{a}, \mathrm{b})$. Motion of a cyclonic vortex over a slope with a positive curvature. 


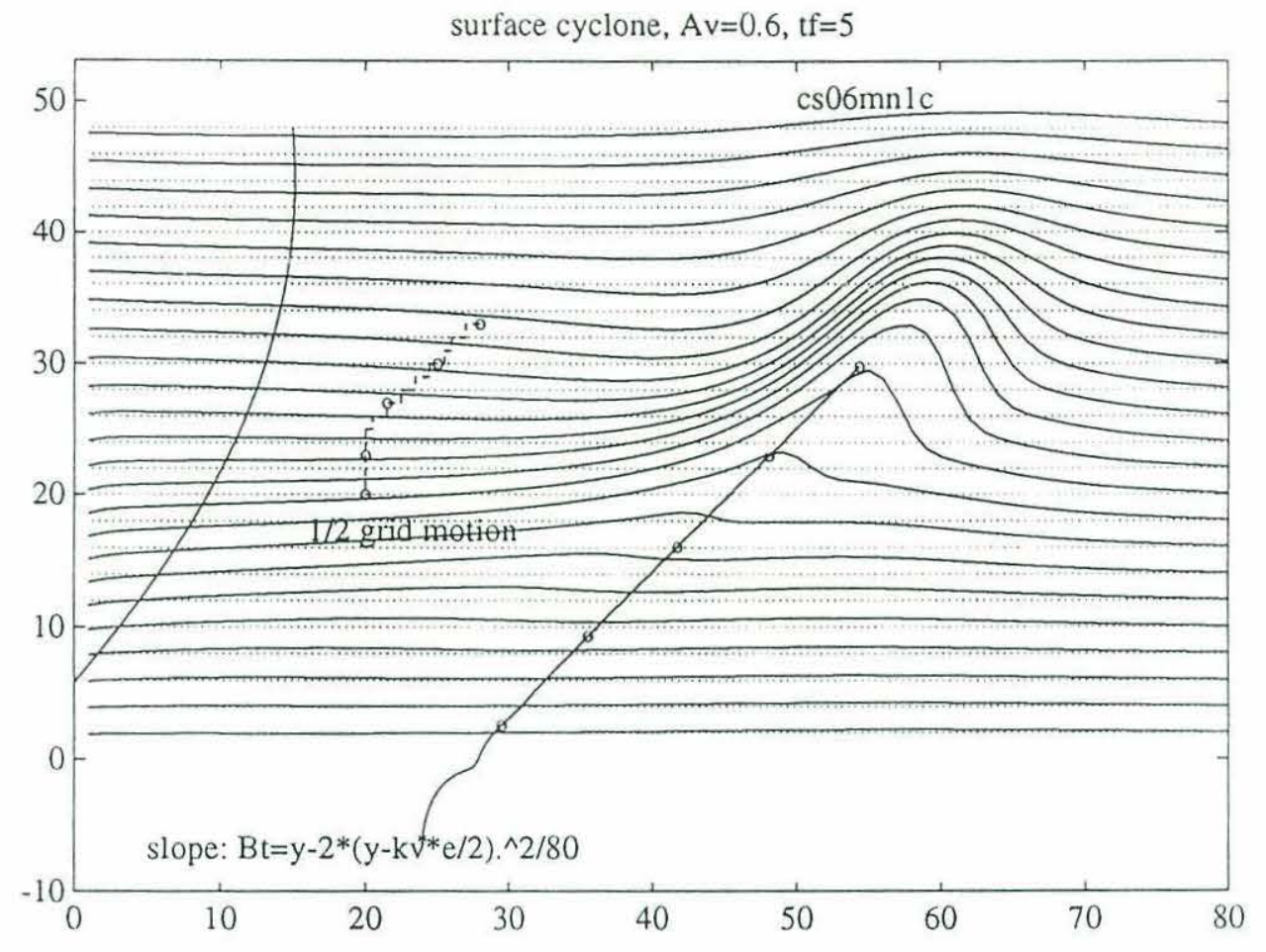

(a)

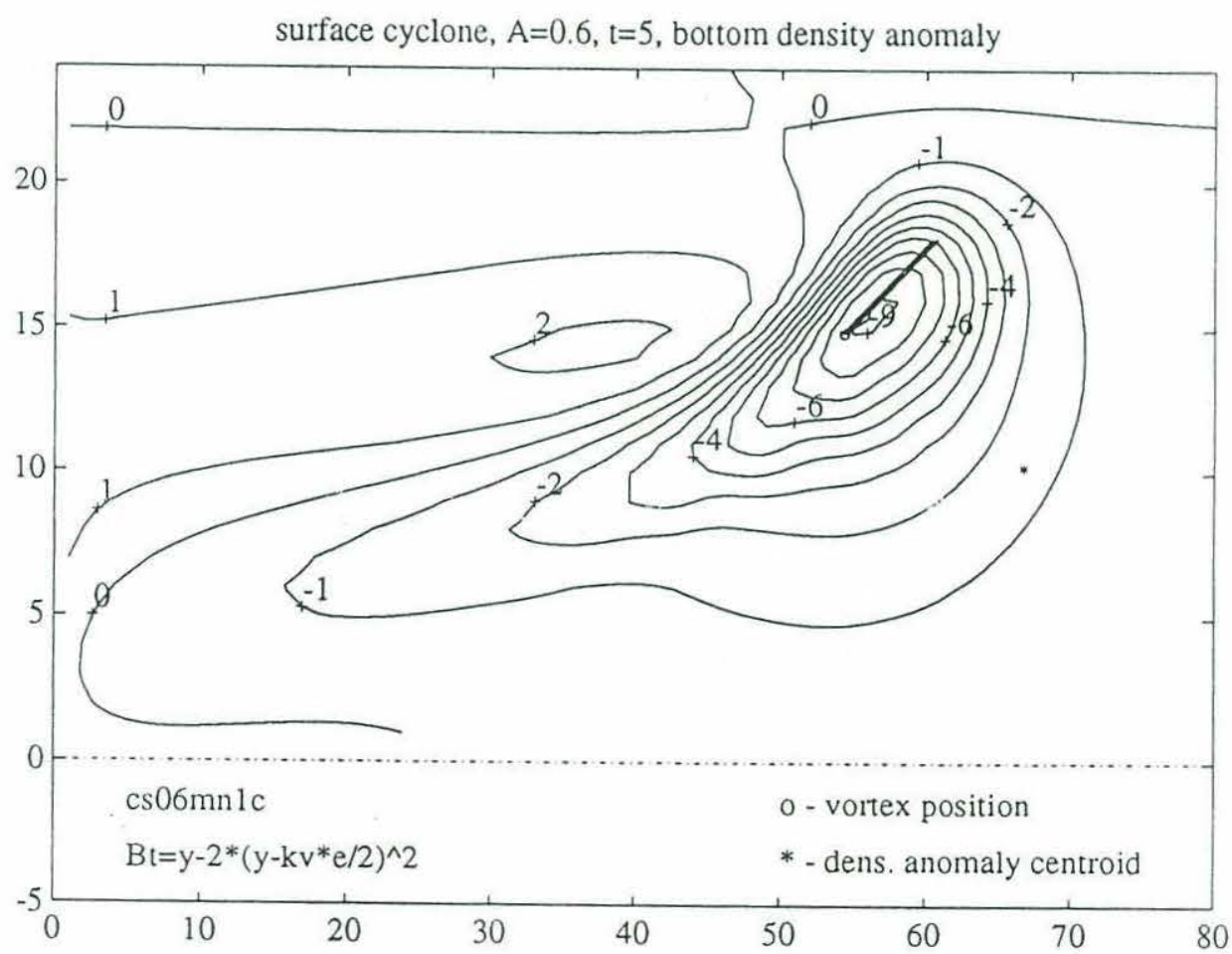

(b)

Figure $6.2(a, b)$. Motion of a cyclonic vortex over a slope with a negative curvature. 


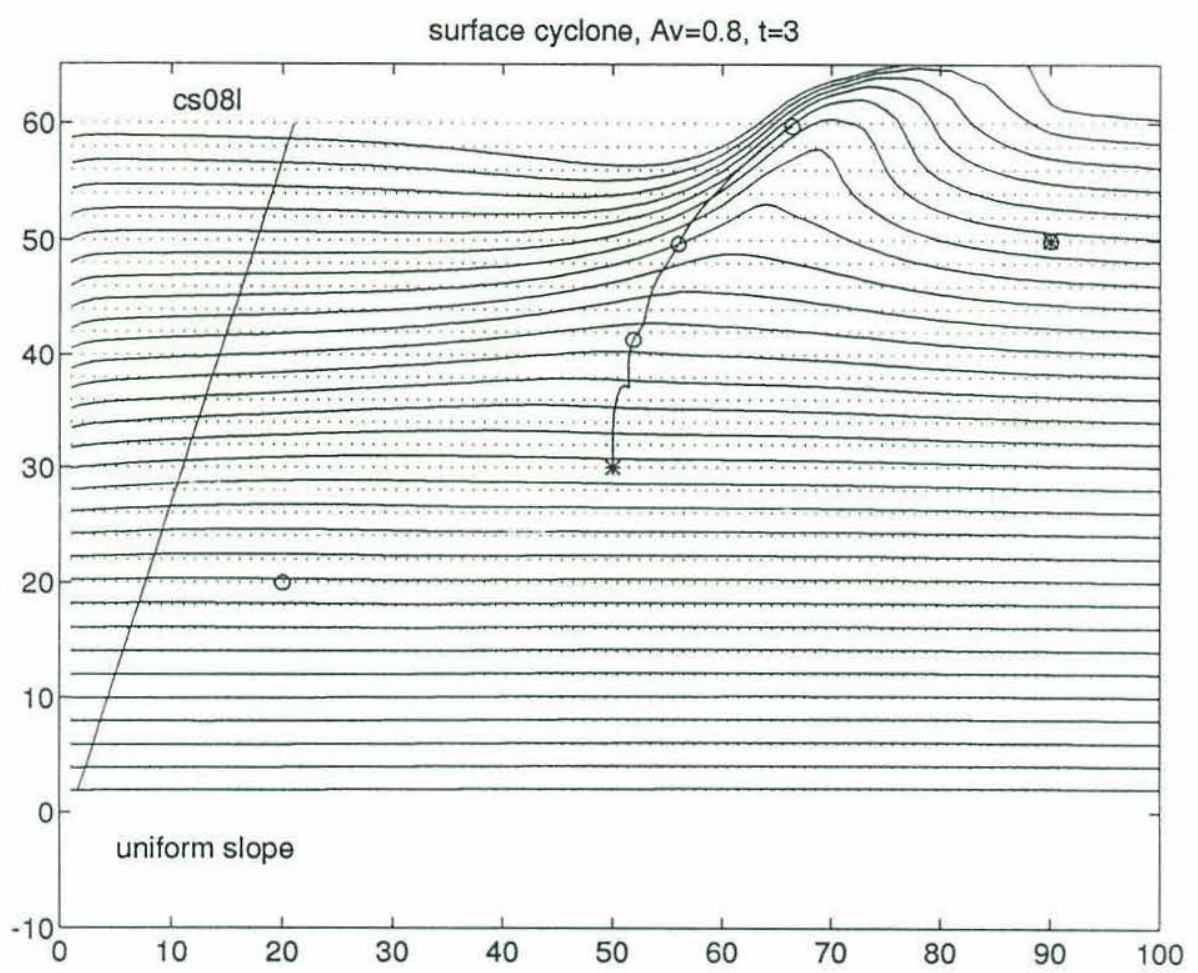

(a)

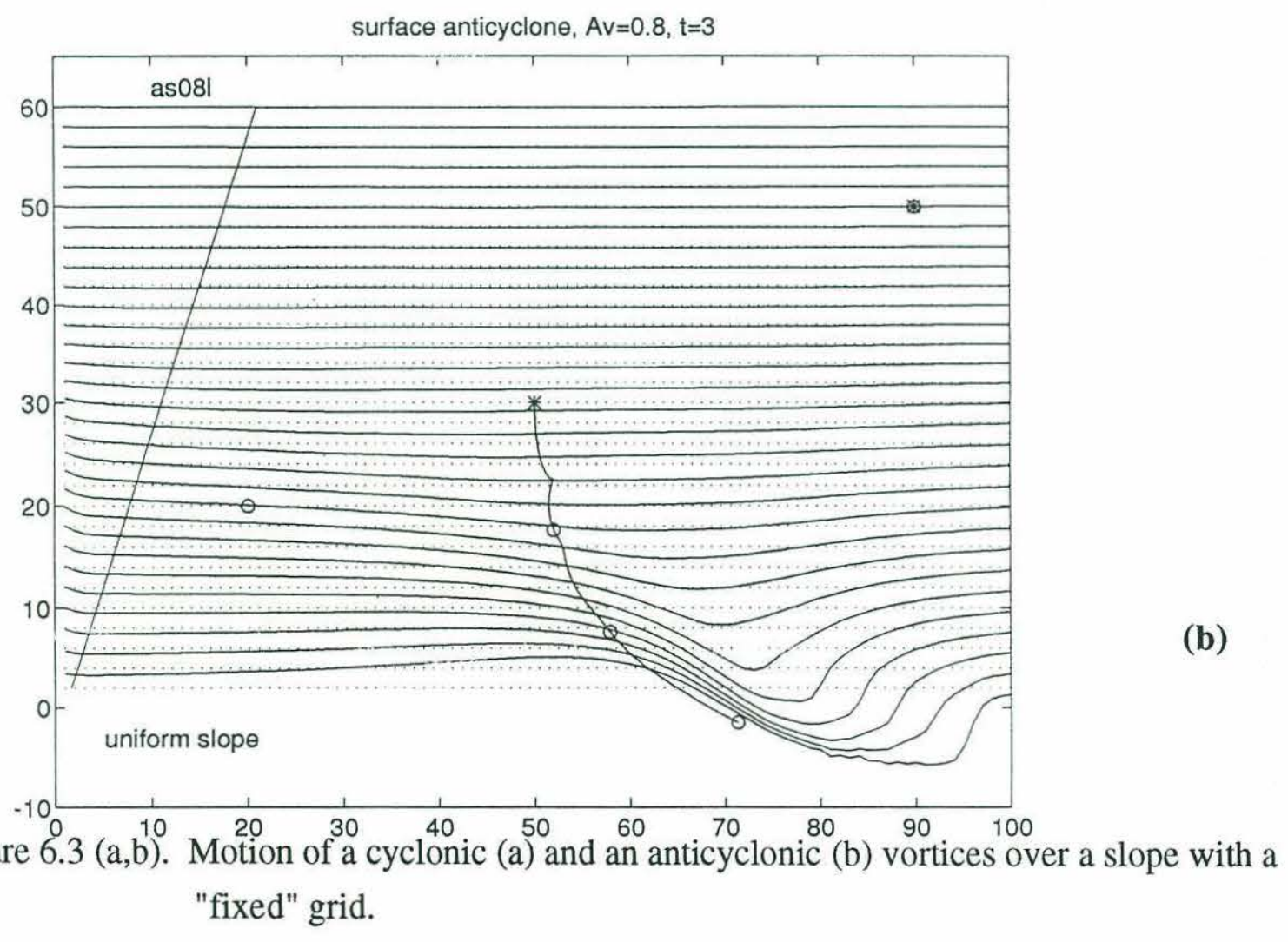


surface cyclone, $\mathrm{Av}=0.8, \mathrm{tf}=2$

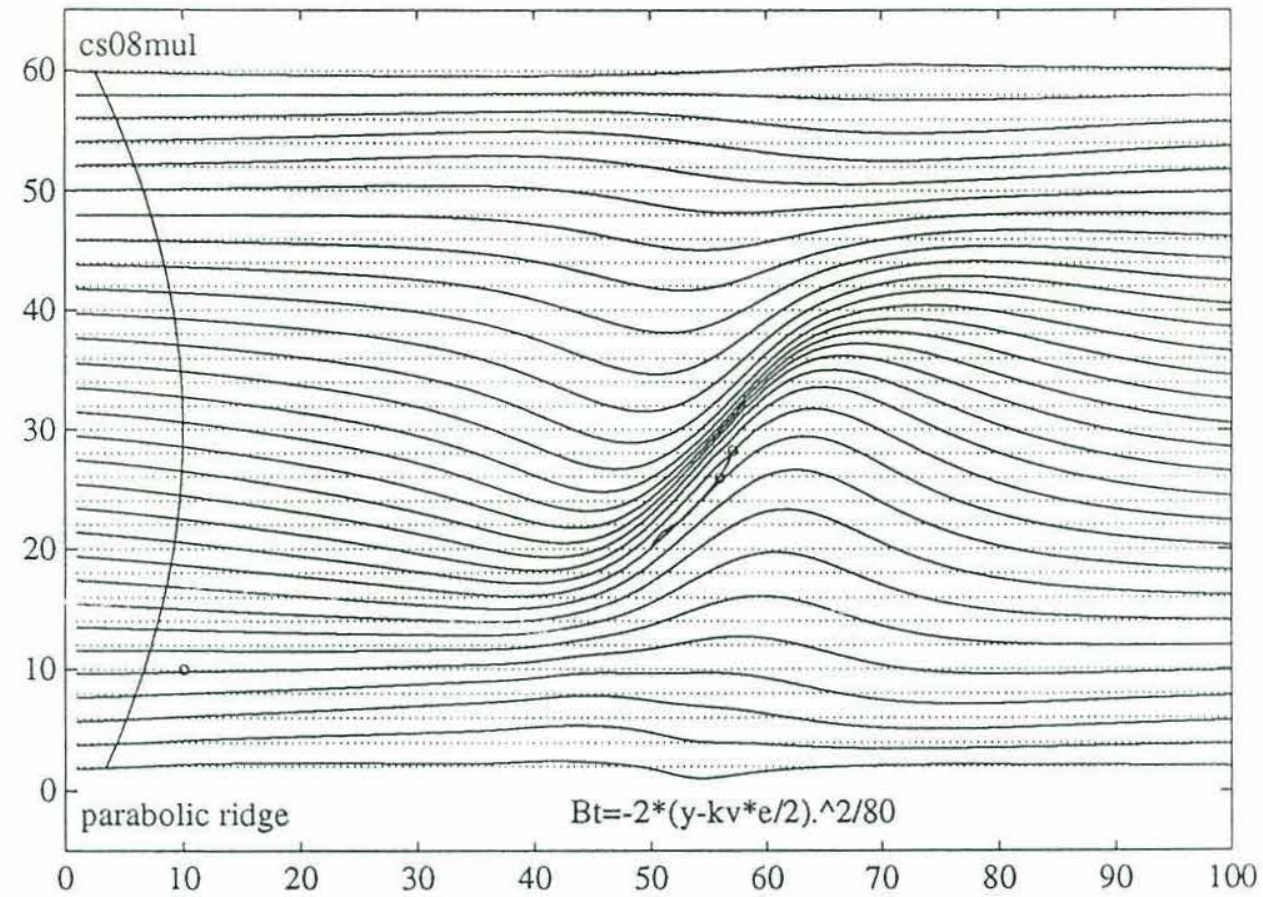

(a)

surface cyclone, $A=0.8, t=2$, bottom density anomaly

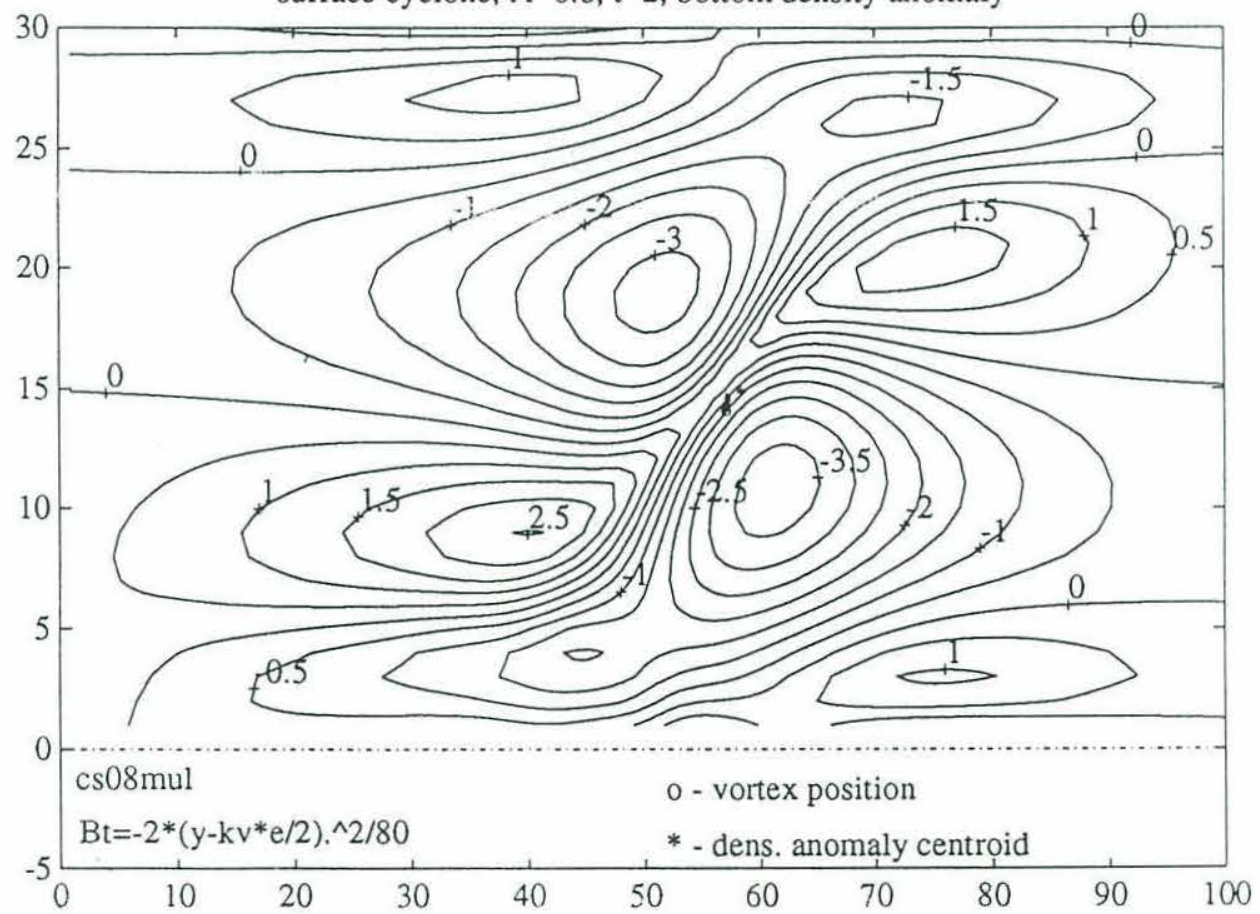

(b)

Figure $6.4(a, b)$. Motion of a cyclonic vortex over the "parabolic hill". 
surface anticyclone, $\mathrm{Av}=-0.8, \mathrm{tf}=3$

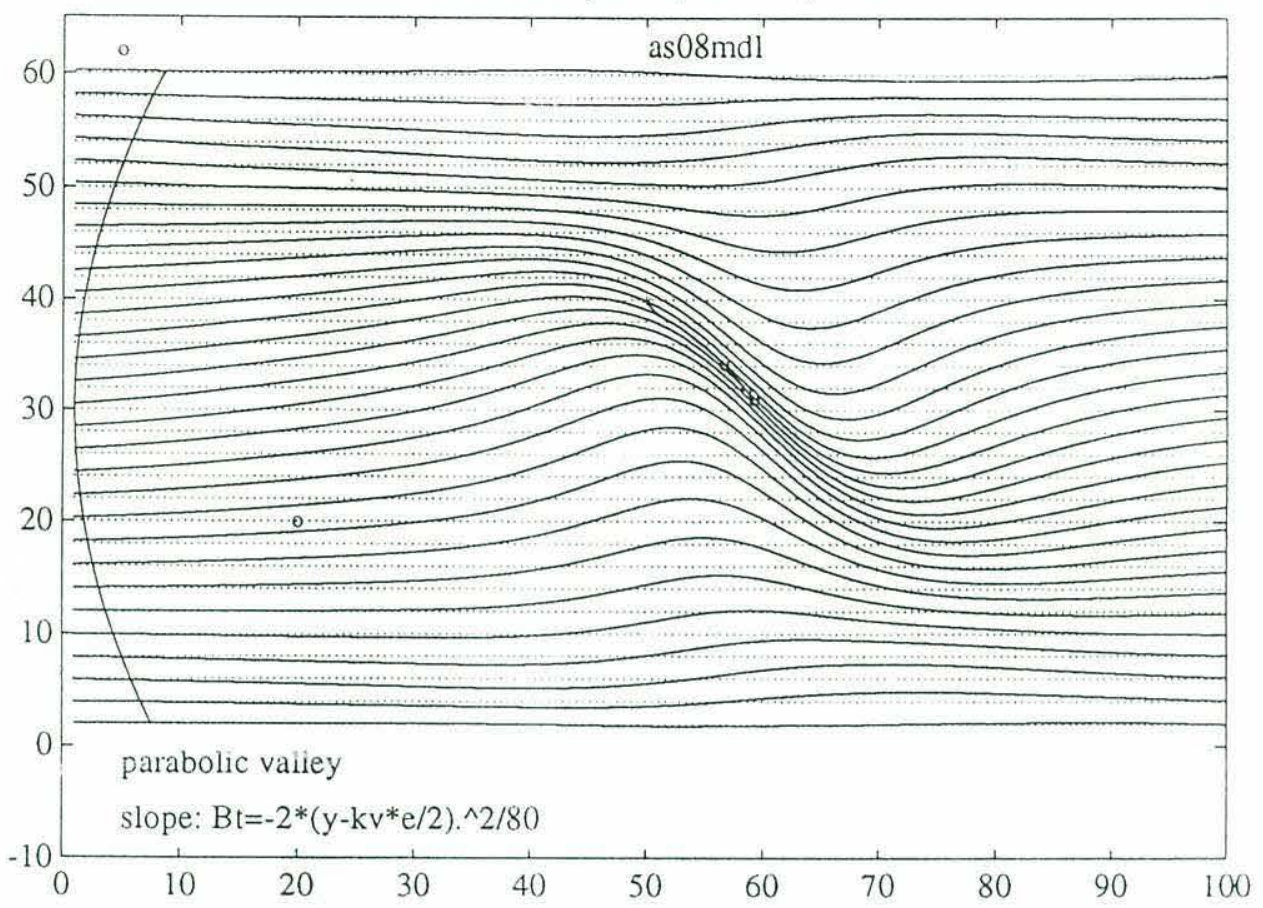

(a)

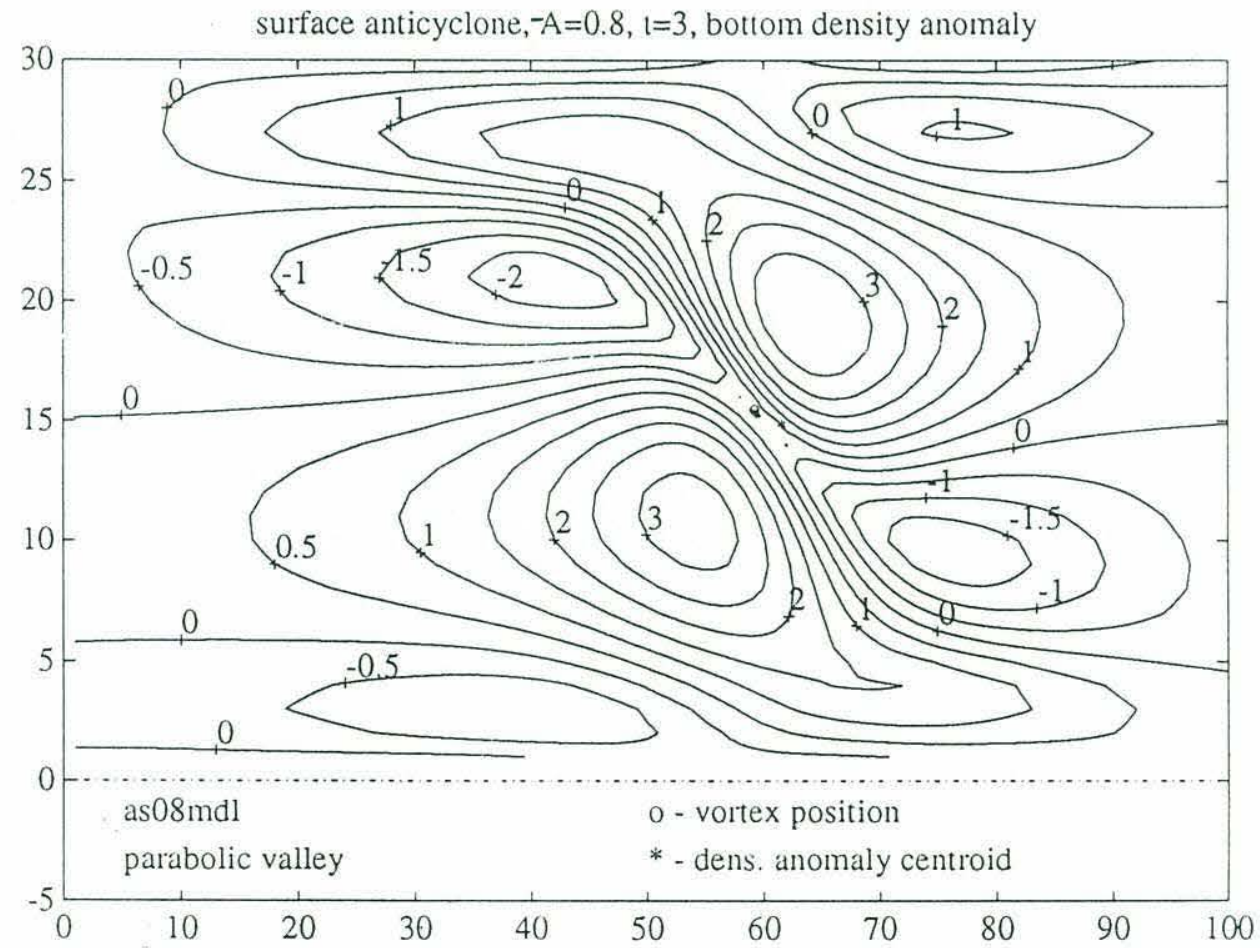

(b)

Figure $6.5(\mathrm{a}, \mathrm{b})$. Motion of an anticyclonic vortex over the "parabolic valley". 
vortices over non-uniform slopes

cyclones anticyclones
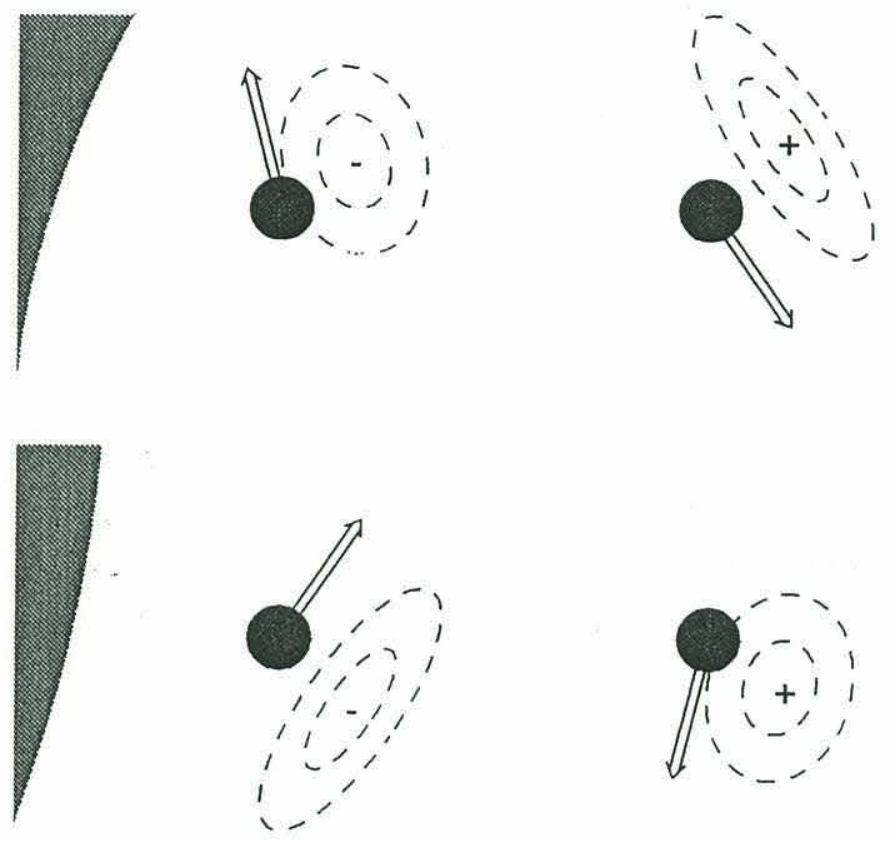

(a)(a)

directions of vortex motion for different topographies
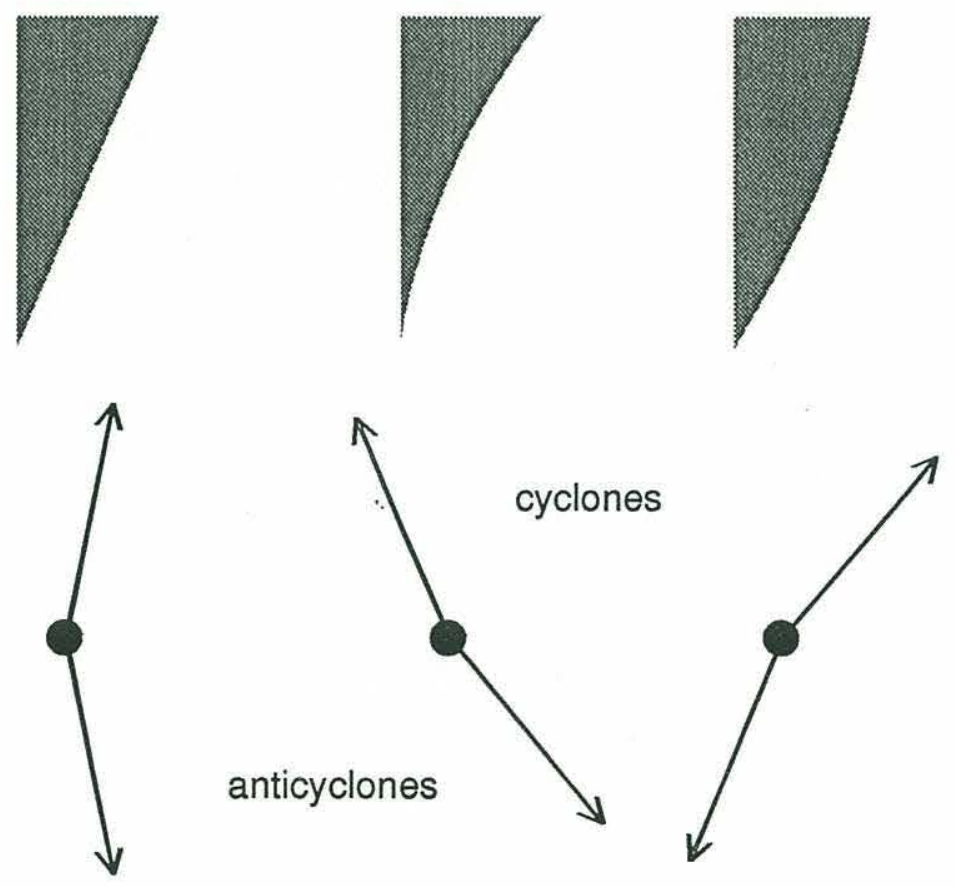

(b) (b)

Figure $6.6(a, b)$. Sketch of directions of vortex motion for different types of topography. 
"horizontal" - the same as for cyclonic vortices (see again the sketch in figure 6.6(a,b) ).

These calculations were performed using the moving grid technique to achieve steady translation. But when studying motion of a vortex over a non-uniform bottom slope the moving grid implies that the bottom itself moves in some sense under a vortex. Therefore it is more consistent to use a fixed grid, with the "flat" upper and lower edges of the domain (such as shown in figure 5.1b). Two examples of vortex motion over a slope with the fixed grid is shown in figure 6.3(a,b) - one for a cyclone and another for anticyclone of the same amplitude.

After an initial impulse a vortex reaches nearly steady motion (although completely steady translation is never achieved with the fixed grid). This later stage (at $\tau=3$ ) is shown in figure 6.3. The trajectory of a vortex is inclined "eastward" for both cyclone and anticyclone, similarly to the case of a cyclonic vortex over a negative curvature slope.

We also performed several experiments over a slope with purely quadratic (parabolic) dependence on the y-coordinate. Such a slope changes sign and has a zero inclination angle in the middle. When a curvature is positive we shall call such bottom topography a "parabolic valley" and in the case of a negative slope - a "parabolic ridge". When a cyclonic vortex is released over a "parabolic ridge" it immediately starts moving towards its upper point (in the middle of the domain). This is shown in figure 6.4 (a). When it reaches the top point is stays there indefinitely in the absence of any background flows or other external factors. Its horizontal position coincides with the coordinates of the centroid of a density anomaly at the bottom. The density anomaly structure (figure 6.4b) has a rather complicated quadrupolar shape with the vortex situated right in the middle of it.

A similar thing happens to an anticyclone over a "parabolic valley" (figure 6.5a,b), although in this case a vortex seeks the lowermost point of the domain. Its velocity decreases gradually as it approaches the middle point with a zero slope angle and finally it stays without motion at this lowest point. The shape of the density anomaly is similar to that of a cyclone over a "parabolic ridge" although it is slanted in the opposite direction. Such behavior of cyclonic and anticyclonic vortices is consistent of course with the general concept that cyclones always move upslope and anticyclones in the downslope direction.

Figure 6.6(a,b), which we mentioned already before, summarizes these results of vortex motion over a straight-line topography. Figure 6.6(a) shows schematically the directions of vortex trajectories and positions of maxima of a bottom density anomaly with positive and negative slope curvatures. When the slope curvature is positive (the inclination angle increases "northward") the density anomaly is shifted upslope (even if the displacement of contours remains the same, which is not exactly the case). Because this density anomaly is equivalent to the opposite-signed circulation it produces an "eastward" (clockwise) component for cyclonic vortices and a "westward" component for anticyclones. For anticyclonic vortices the anomaly maximum (and the centroid) is shifted further from a vortex itself and the alongslope ("eastward") component is somewhat larger than the 
"westward" velocity for cyclones over the same slope. When a slope curvature has a negative sign the situation is opposite - cyclonic vortices have an "eastward" component of velocity, while trajectories of anticyclones are inclined slightly "westward". The "eastward" velocity component is always stronger for the same setting than the "westward" one, most probably because vortices have already a small "eastward" velocity even with a constant-angle slope. In figure 6.6(b) the directions of vortex motion are sketched for all three types of straight-line topography: with zero, positive and negative slope curvatures. It repeats essentially our previous pictures and is presented here for easy reference.

In the case of topographic irregularities situation can be more complicated. If these perturbations have a characteristic horizontal scale larger than a "footprint" which a vortex induces in the bottom density field one can expect that they will not influence its dynamics significantly, except that our previous results will apply to the "local" cross- and along-slope directions. But the size of this "footprint" is large enough to cover an area over which topography can vary considerably. Its characteristic scale is actually larger than the "stretched" depth of an ocean, and much larger than the deformation radius. For a surface vortex in the ocean 2-4 km deep this corresponds to an area of approximately $100-200 \mathrm{~km}$ in diameter which can certainly contain many topographic features of smaller scales.

I tried to study the influence of such features by superimposing smaller-scale perturbations over the straight-line $\mathrm{x}$-independent slope in the model. I do not attempt however to study very rough topography - seamounts and ridges, where quasigeostrophy may not be valid, so perturbations of isobatic contours are not very big and these contours are not closed within the domain. I present here four examples of vortex trajectories over different types of topographic perturbations. For these experiments I plotted only trajectories of vortices and current positions of density contours without contour plots of density anomalies. Bathymetric contours are shown by the dotted lines. In figure 6.7(a,b) two experiments with the cyclonic vortex are shown. In figure 6.7(a) a "northward"- going vortex over a uniform slope encounters two topographic "troughs" consecutively. One can see that the trajectory is curved approximately in accordance with the local "northward" direction. Its average velocity remains practically unchanged. There is also some "westward" drift in comparison with the "eastward" component over an unperturbed slope. Figure 6.7(b) shows the experiment with a slightly different type of topographic perturbation: the slope has some positive curvature and there is a "canyon" in the middle of the picture - the long and narrow trough in the cross-slope direction. The cyclonic vortex of the same intensity $(\widehat{\mathrm{A}}=0.8)$ is released on the right ("eastern") side of the canyon. It goes quickly to the "western" side and climbs "northward" along the left side of the canyon, but it does not escape out of the trough. Note a 
surface cyclone, $\mathrm{Av}=0.8, \mathrm{tf}=3$

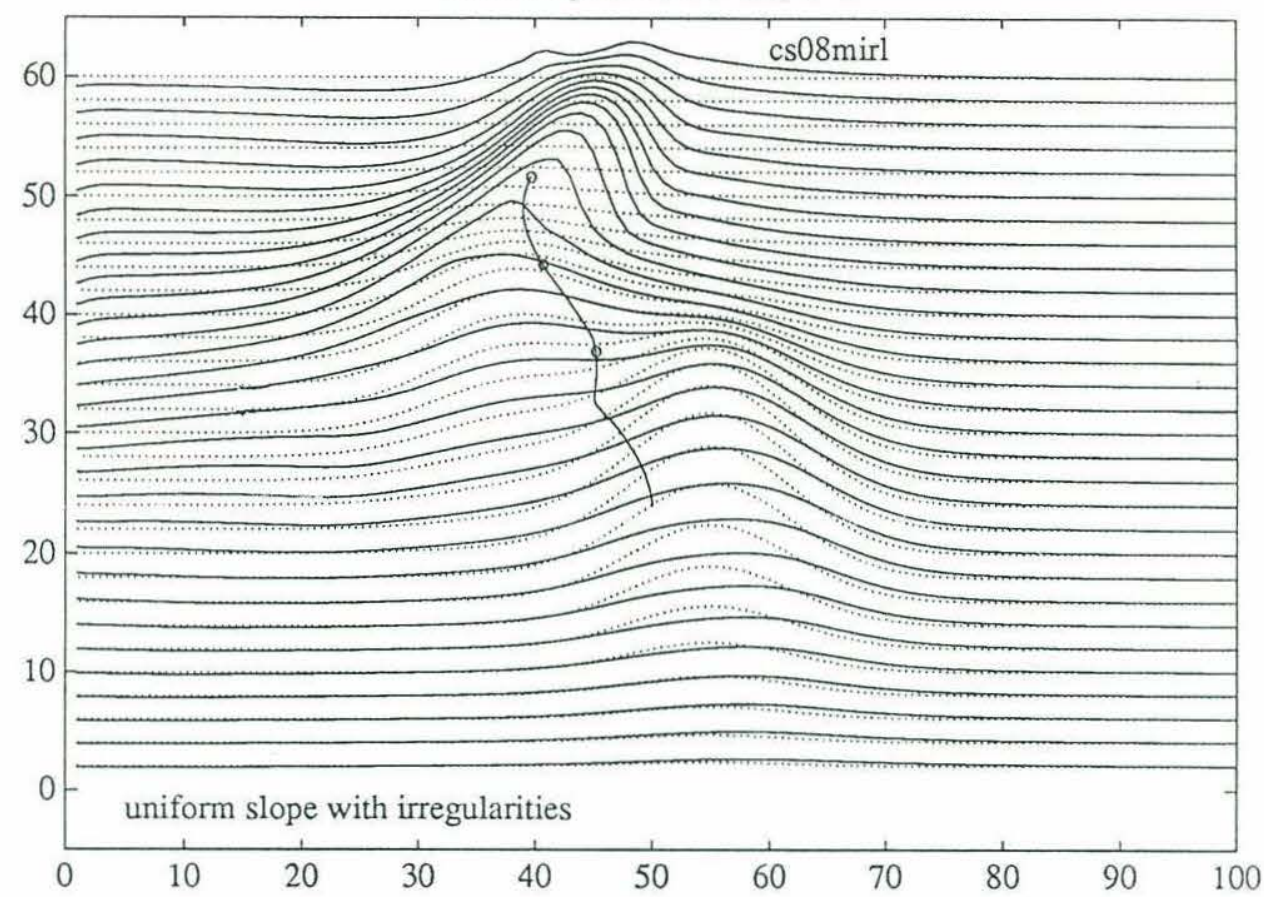

(a)

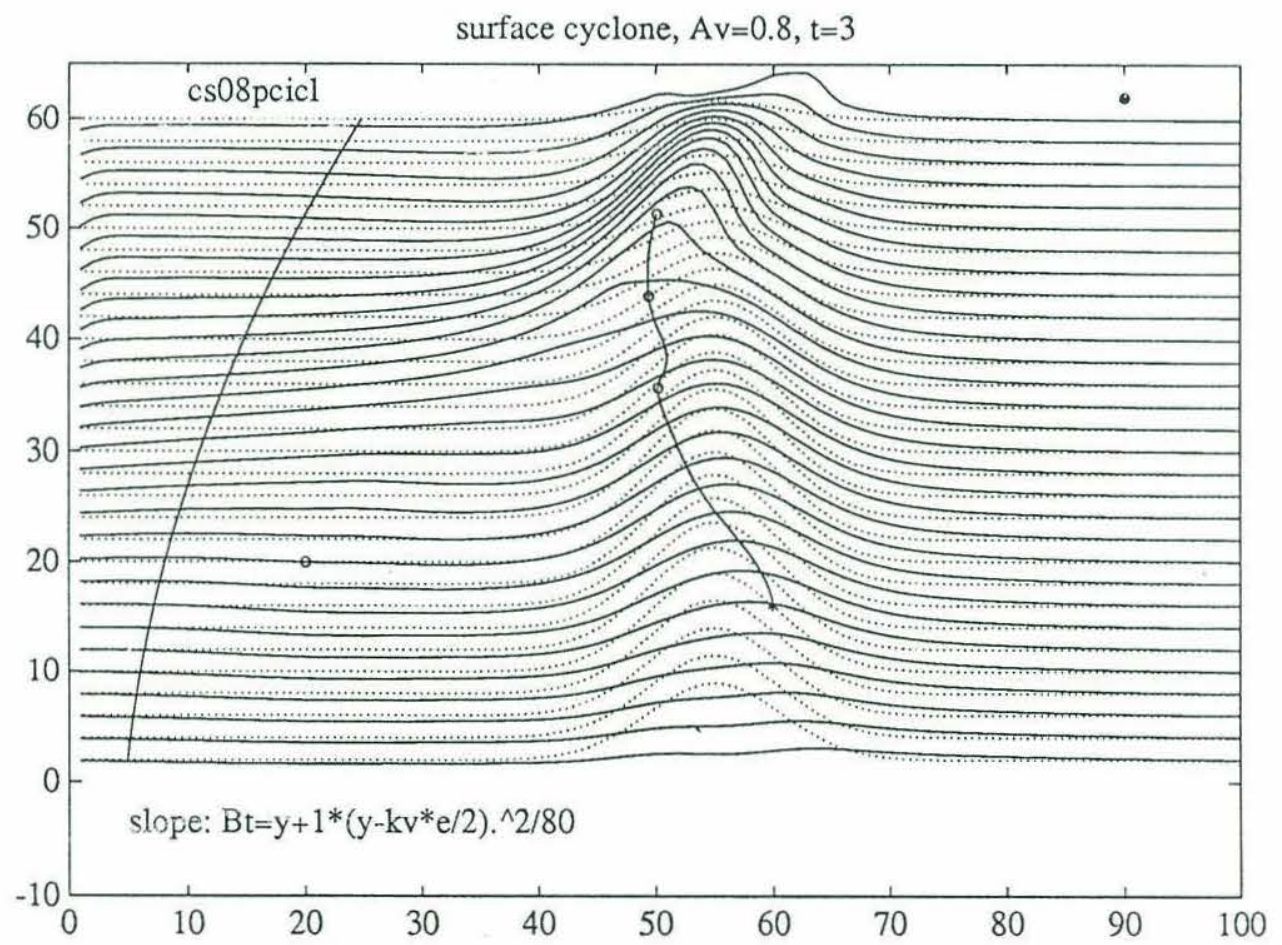

(b)

Figure $6.7(a, b)$. Motion of a cyclonic vortex over the slope with irregularities. 
surface anticyclone, $\mathrm{Av}=-0.8, \mathrm{t}=5$

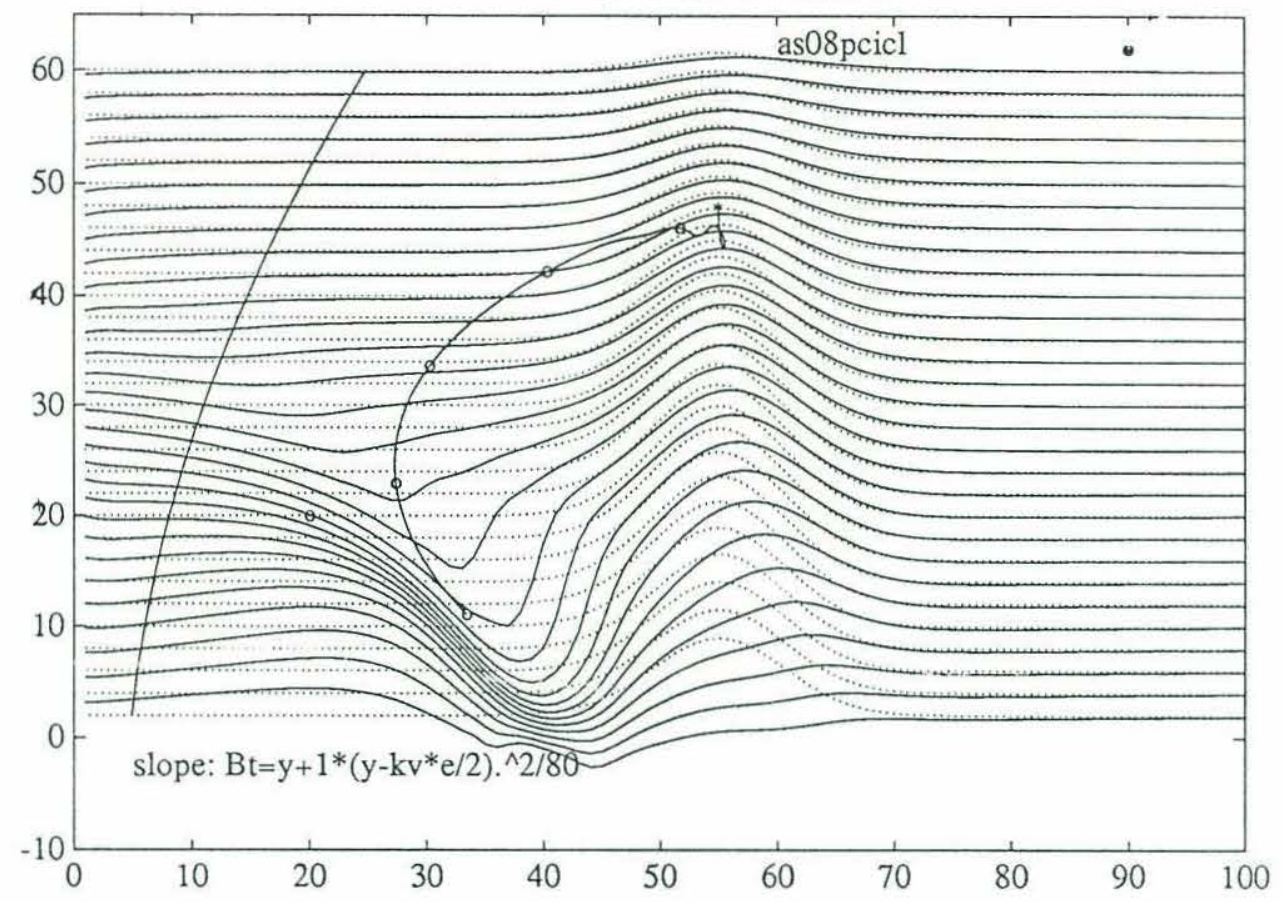

(a)

surface anticyclone, $\mathrm{Av}=-0.8, \mathrm{t}=5$

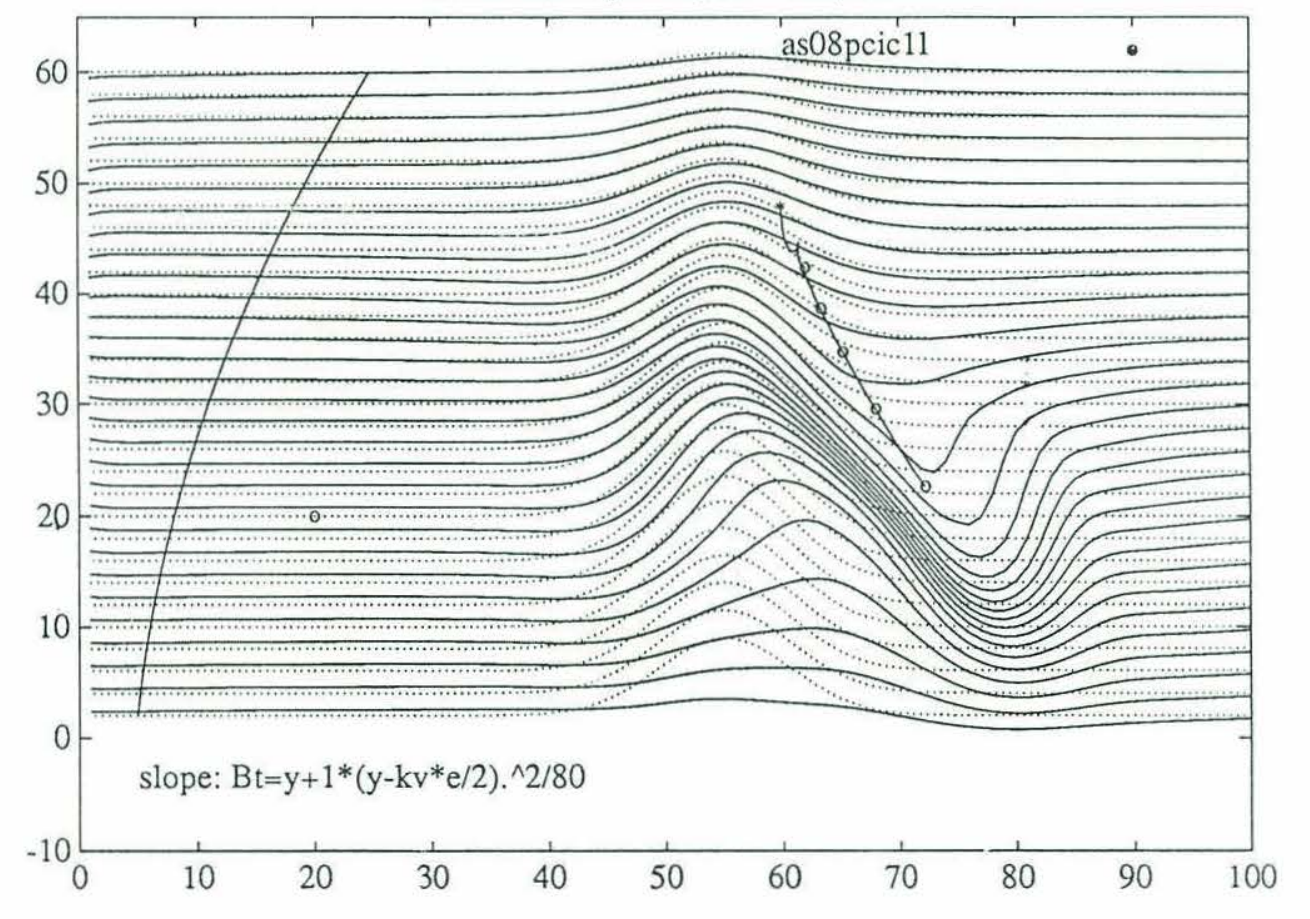

(b)

Figure $6.8(\mathrm{a}, \mathrm{b})$. Motion of an anticyclonic vortex over the slope with the "canyon". 
very rapid initial acceleration in the "north-western" direction, accompanied (not shown in the picture) by a strong surge of topographic waves. Its trajectory does not seem to reach a completely steady state and exhibits some wiggles and perturbations.

The more interesting example is shown in the next figure 6.8(a,b) where two identical-twins experiments with the anticyclonic vortex over the same slope with the canyon are shown. The only difference between these two runs is the initial position of a vortex: in figure 6.8(a) it starts from the middle of the trough and in figure 6.8(b) its position is shifted slightly to the right, on the "eastern" slope. But this small difference in the initial position leads to a dramatic change in its trajectory: When released over the center it escapes to the left, moves rapidly in the almost along-slope "western" direction until getting far enough from the trough. Then its path starts curving to the "south-east" according to previously described case of a slope with positive curvature. But when it starts from the "eastern" side of the canyon it moves in the "south-eastern" direction from the beginning, although its speed is much slower in this case. It may seem surprising that the anticyclonic vortex tends to escape the trough, contrary to its propensity of moving toward the deepest part of the domain. While this "southward" tendency remains the same, small-scale topographic features can apparently cause significant changes in its trajectory.

It is clear from these examples that the presence of small-scale topographic variations can complicate the simple picture presented above of a vortex motion over a uniform or curved slope. However the most important feature - the cross-slope tendency (cyclones - "northward", anticyclones-southward") - remains unchanged. But the zonal, along-slope velocity can differ dramatically in various cases and sometimes exceed the cross-slope component. There are some observational evidences of the influence of bathymetric irregularities on vortices: for example trajectory of the warm-core ring 82B was significantly perturbed when it passed the Hudson canyon region (see figure 1.1a). Apparently this issue deserves a further study. We would not however elaborate on this problem in the present work and instead consider some other aspects of the vortextopography interaction.

\subsection{Presence of background flows}

Among the different factors influencing vortex motion the advection by background flows should be one of the most important. In the absence of topography, planetary beta-effect and other forces that could contribute to the self-propagation, vortices will simply be advected by an external 
flow with its velocity in the middle of a vortex. On the other hand a combination of some important self-propelling mechanism, like bottom topography, and a large-scale flow can result in the advection velocity which is different from that of in the presence of only one of these factors.

In this section we want to examine the influence of a large-scale baroclinic, surface-intensified flow on the vortex-topography interaction. The reason for this is its relative simplicity. A background barotropic flow will influence both vortex and bottom density field simultaneously, which can result in rather complicated dynamics. Moreover, such barotropic flow can have only an along-slope direction, otherwise it will result in systematic advection of density contours across the slope which is rather difficult to interpret in our model. Instead we choose a baroclinic flow with a constant vertical shear and a uniform potential vorticity (and $\beta=0$ ), which will have a zero velocity at the bottom. Thus it will influence directly only a vortex itself, and not the bottom density contours. This is a crude model of a large-scale surface-intensified flow. It is not consistent however to consider this problem in such a simplified treatment in the presence of the surface, because generally we must specify a density field at the surface and compute its evolution explicitly. Without the background shear one can set the density perturbation at the surface to be zero and in this case it will not change in time because of the rigid-lid condition. But if one adds a flow with a uniform vertical shear and a constant potential vorticity it will lead to the surface density perturbation which is linear in the cross-flow coordinate. In this case one must follow the evolution of density contours at the surface, which is possible of course but requires some extension of the present model.

But I choose a simpler approach here: I discard the surface completely and consider a deep vortex far below the surface. This can be not very realistic but at least a consistent approximation. However crude and inadequate such a parameterization of background flow in our model, I consider it useful to study some possible effects of the combination of advection with bottom topography.

For this series of our calculations we chose a constant flow perpendicular to the slope. We experimented with flow velocities at the level of a vortex ranging from very weak to much stronger than the characteristic velocity of the vortex $\mathrm{v}_{0}$. The results of some of our runs are presented in figures $6.9-6.14$. In addition to our usual notation and plotting technique we also show the direction and strength of a flow by a dashed line in the corner of our upper plots. Such a line shows actually the advection by the flow of a "free" water parcel far from a vortex in the course of each run. Sometimes when the flow is very strong we reduce this line by a factor of 2 and add a corresponding comment like "1/2 flow" near these lines.

Figure 6.9(a,b) is a reference picture and shows the vortex trajectory and the bottom density structure for a deep cyclone (without the surface) without any background flow. The vortex shows 


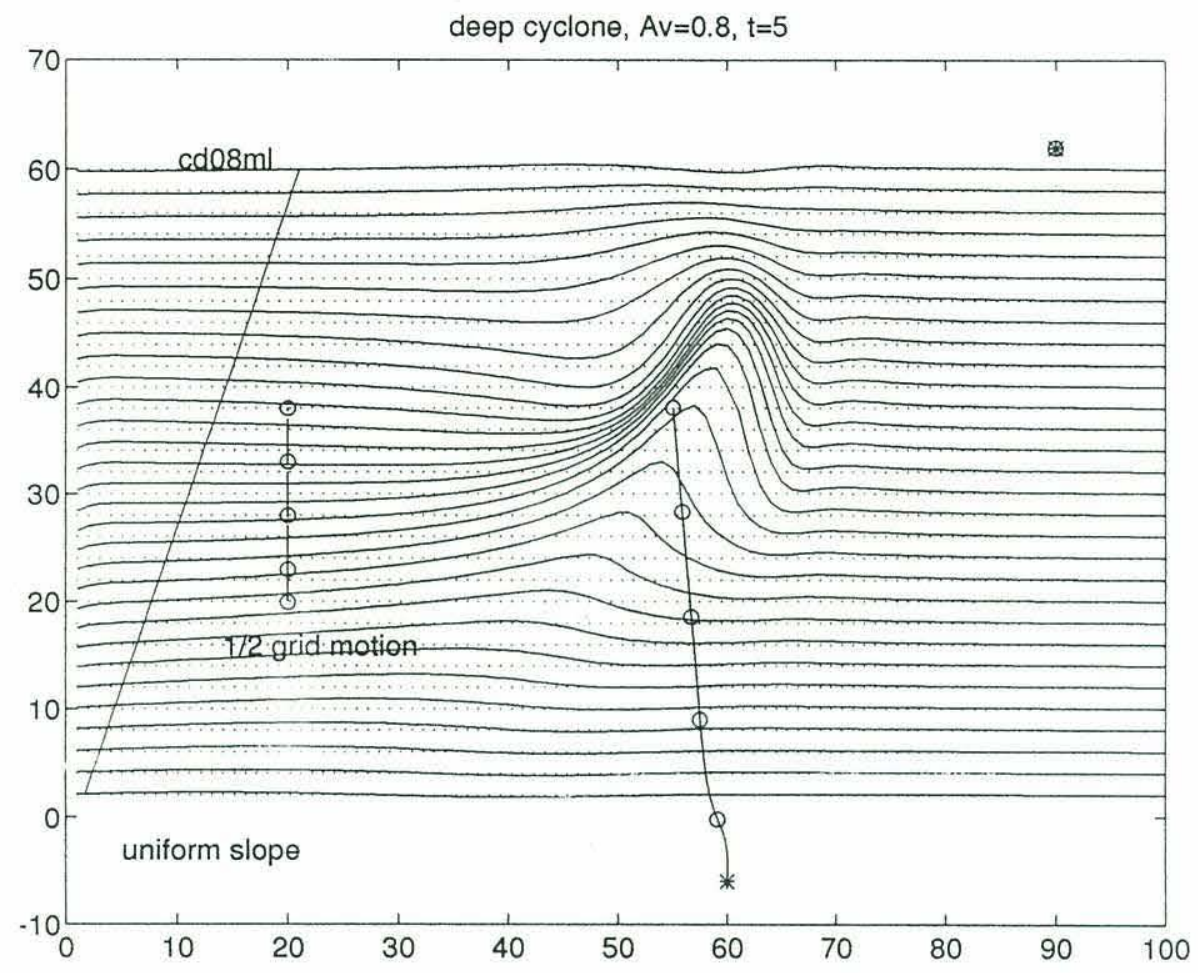

(a)

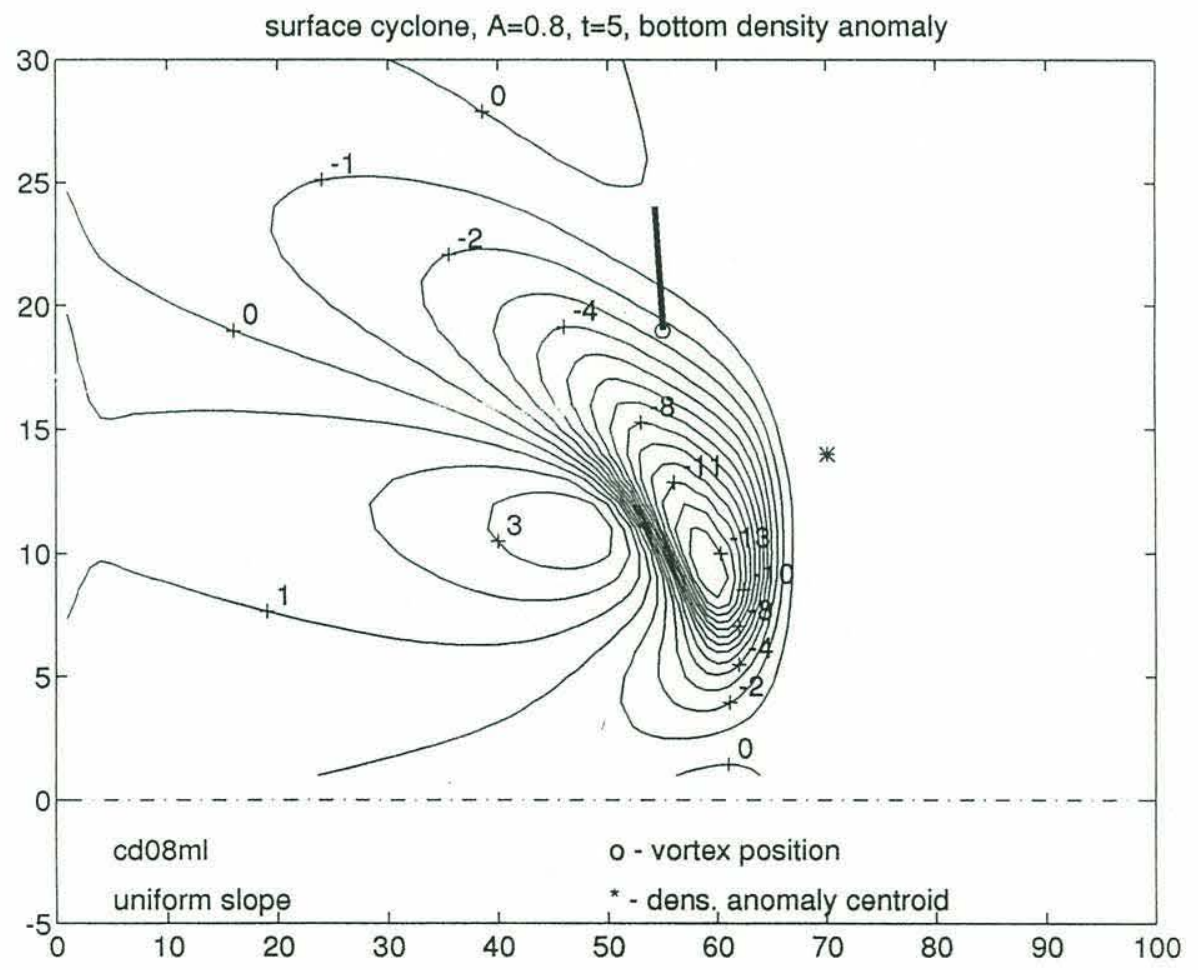

(b)

Figure $6.9(a, b)$. Motion of a deep cyclonic vortex over the uniform slope without the background flow 


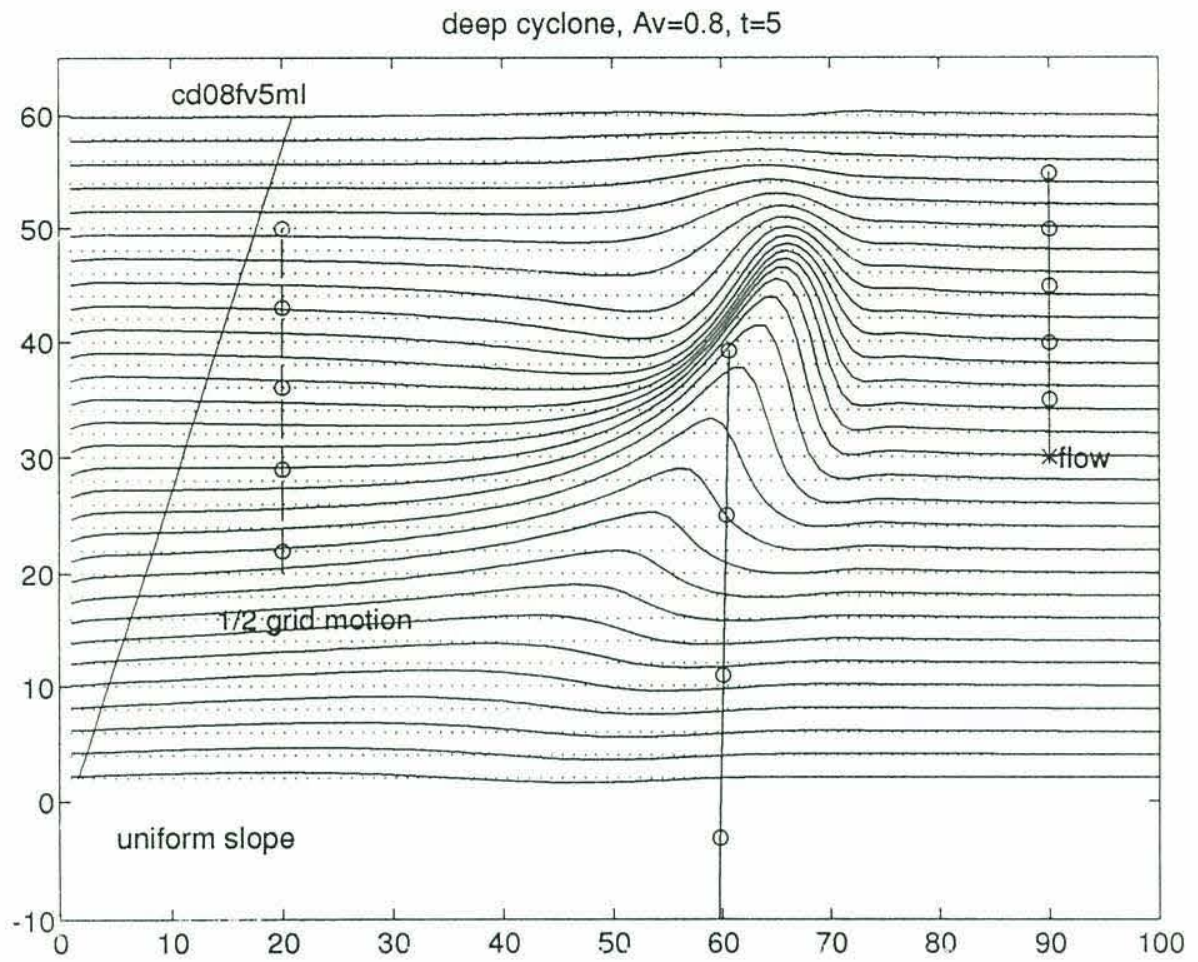

(a)

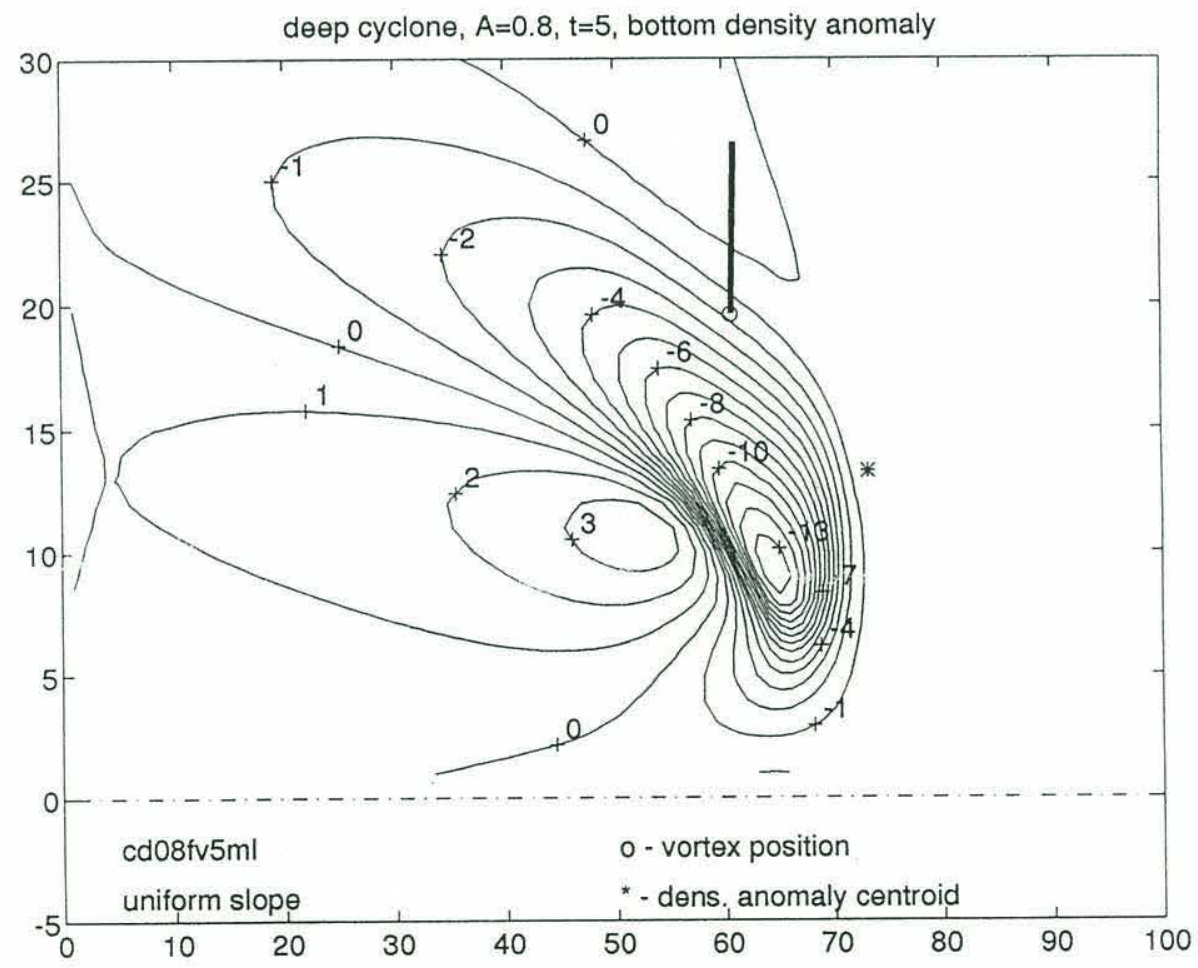

(b)

Figure $6.10(a, b)$. Motion of a cyclonic vortex over the uniform slope with the background "northward" flow, $v_{\text {flow }}=5$. 


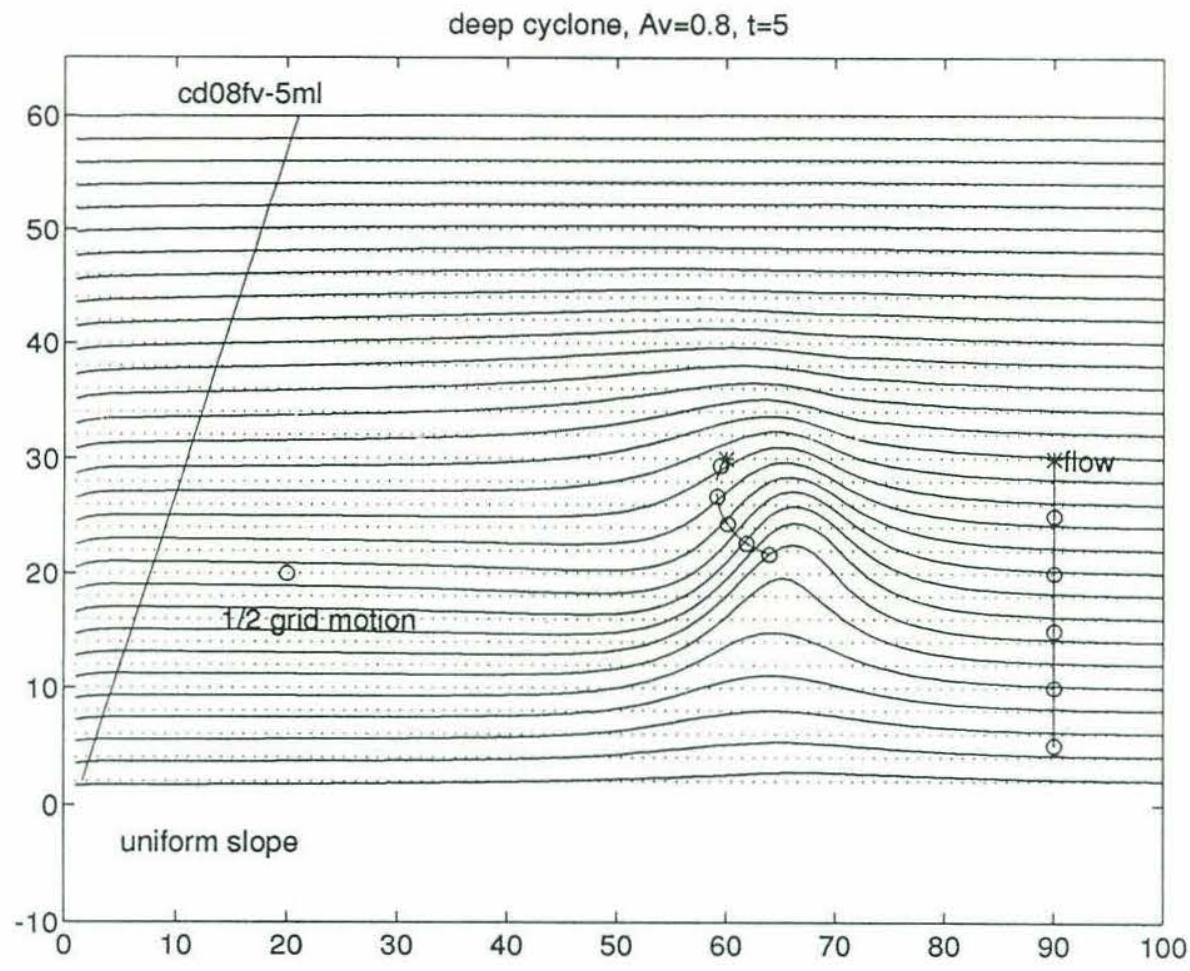

(a)

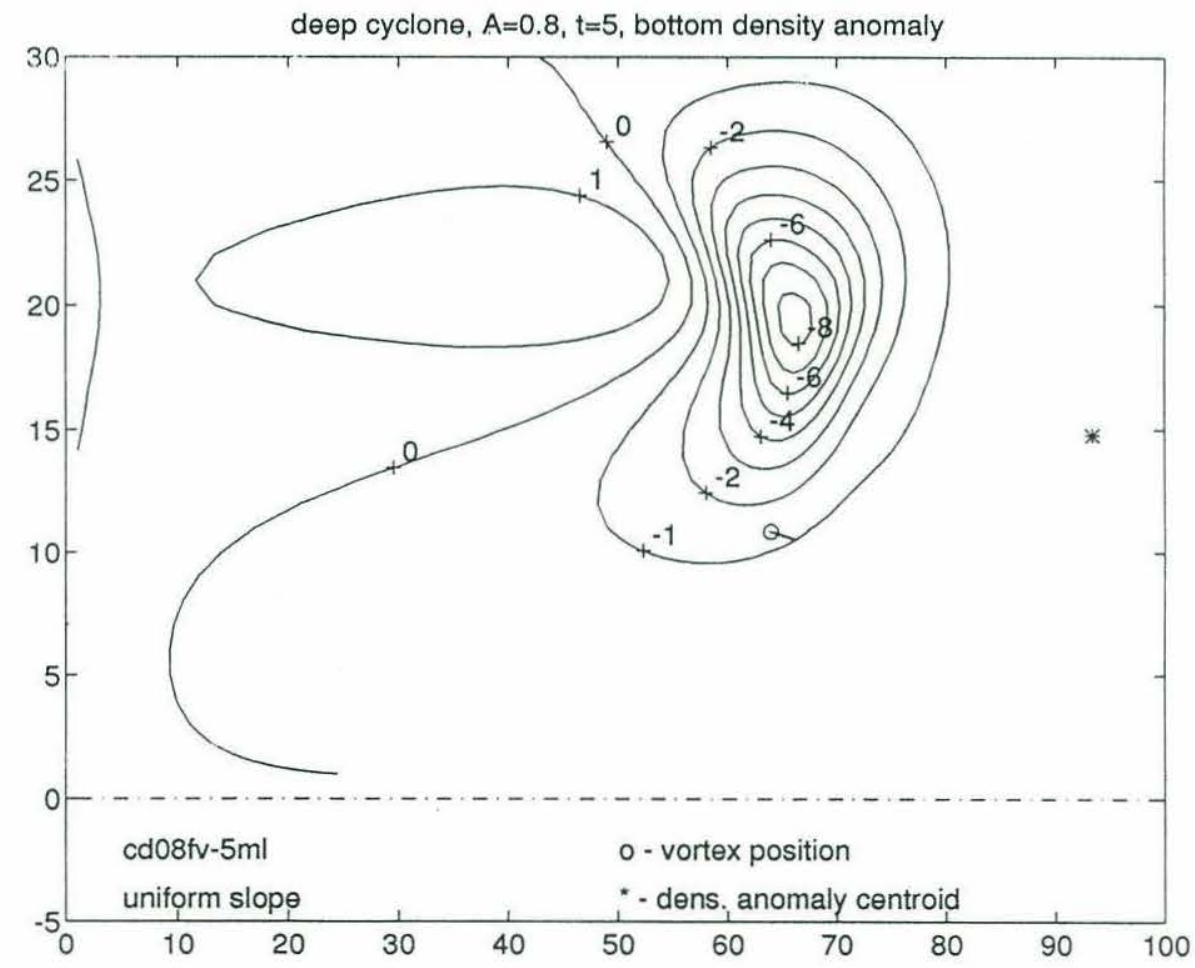

(b)

Figure $6.11(\mathrm{a}, \mathrm{b})$. Motion of a cyclonic vortex over the uniform slope with the background "southward" flow, $v_{\text {flow }}=-5$. 


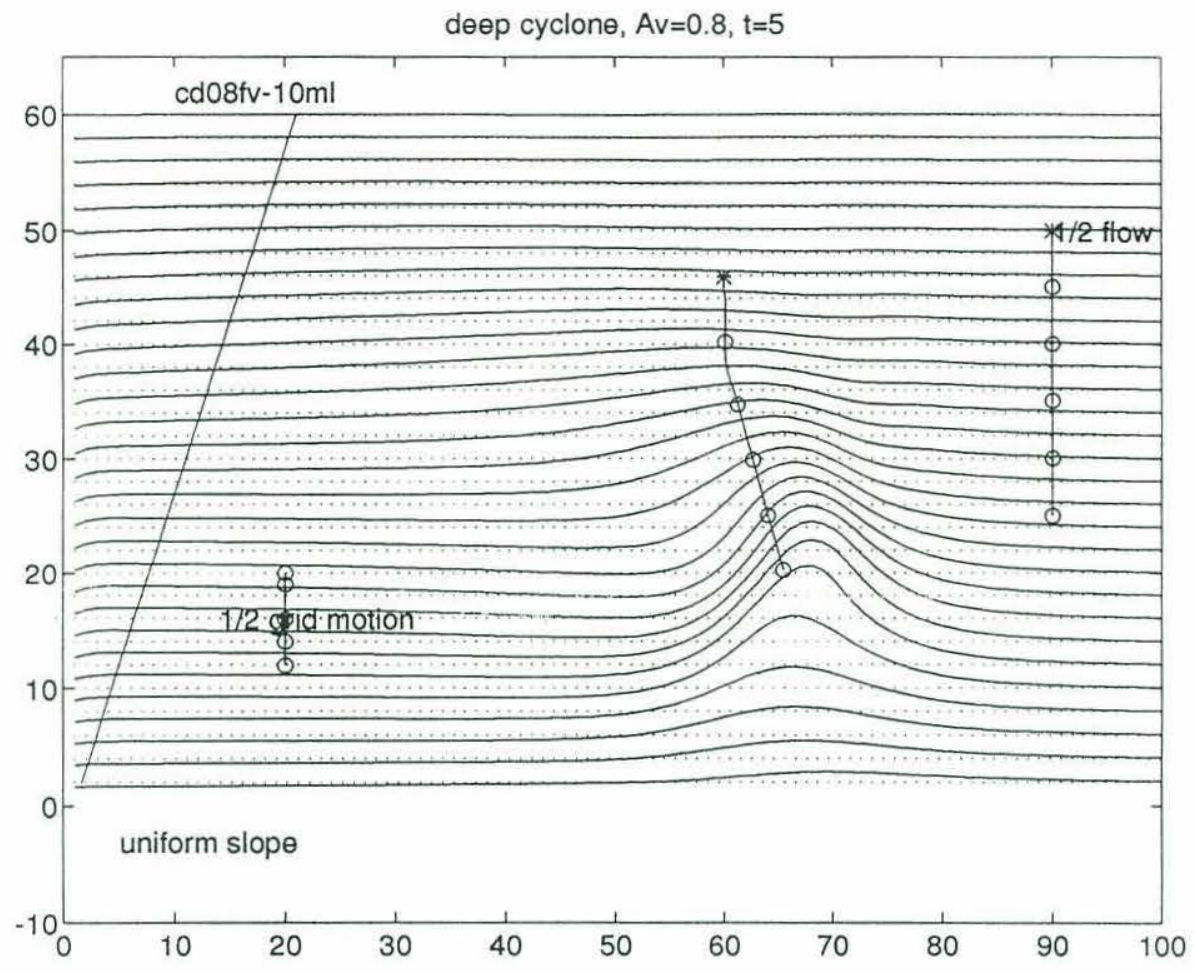

(a)

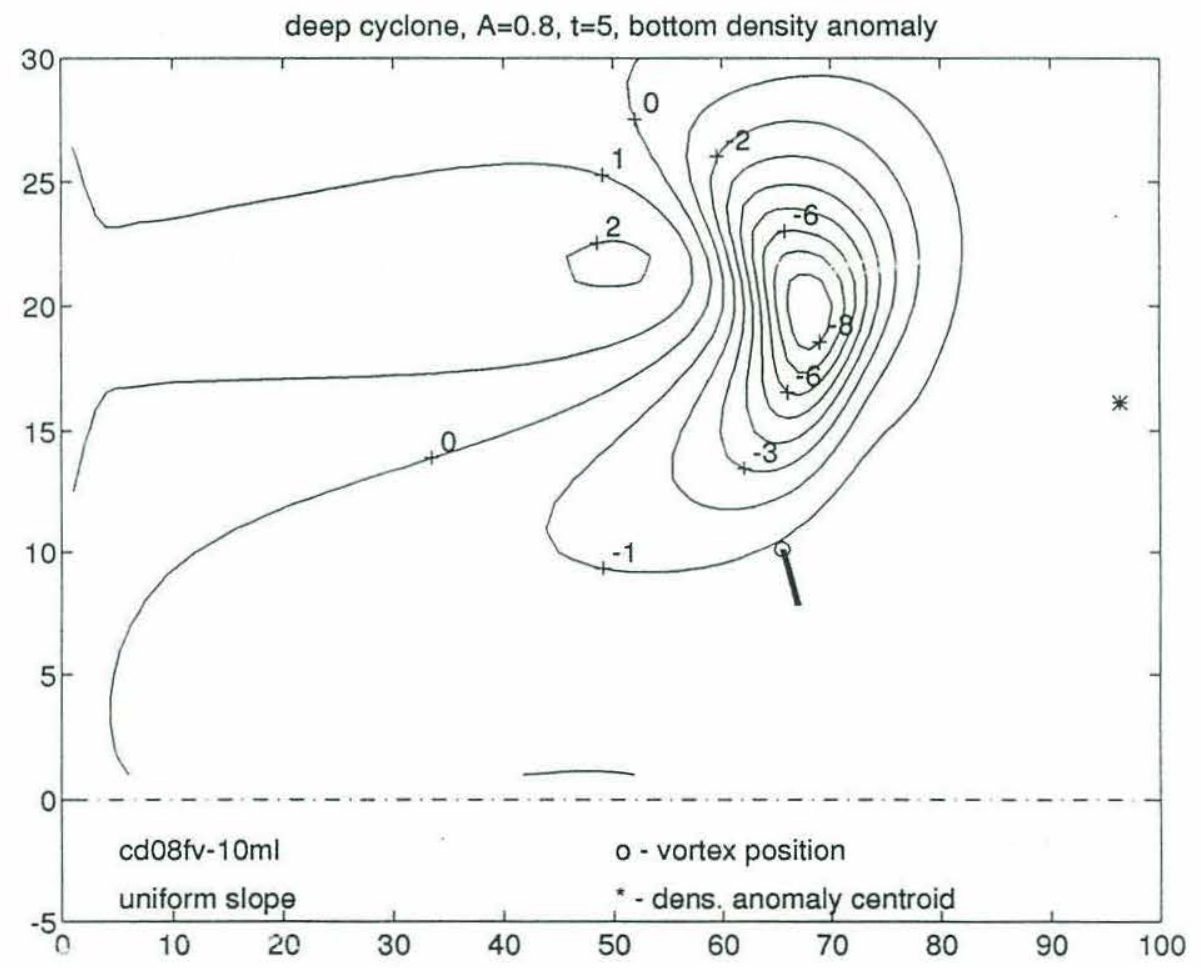

(b)

Figure $6.12(a, b)$. Motion of a cyclonic vortex over the uniform slope with the background "southward" flow, $\mathrm{v}_{\text {flow }}=-10$. 


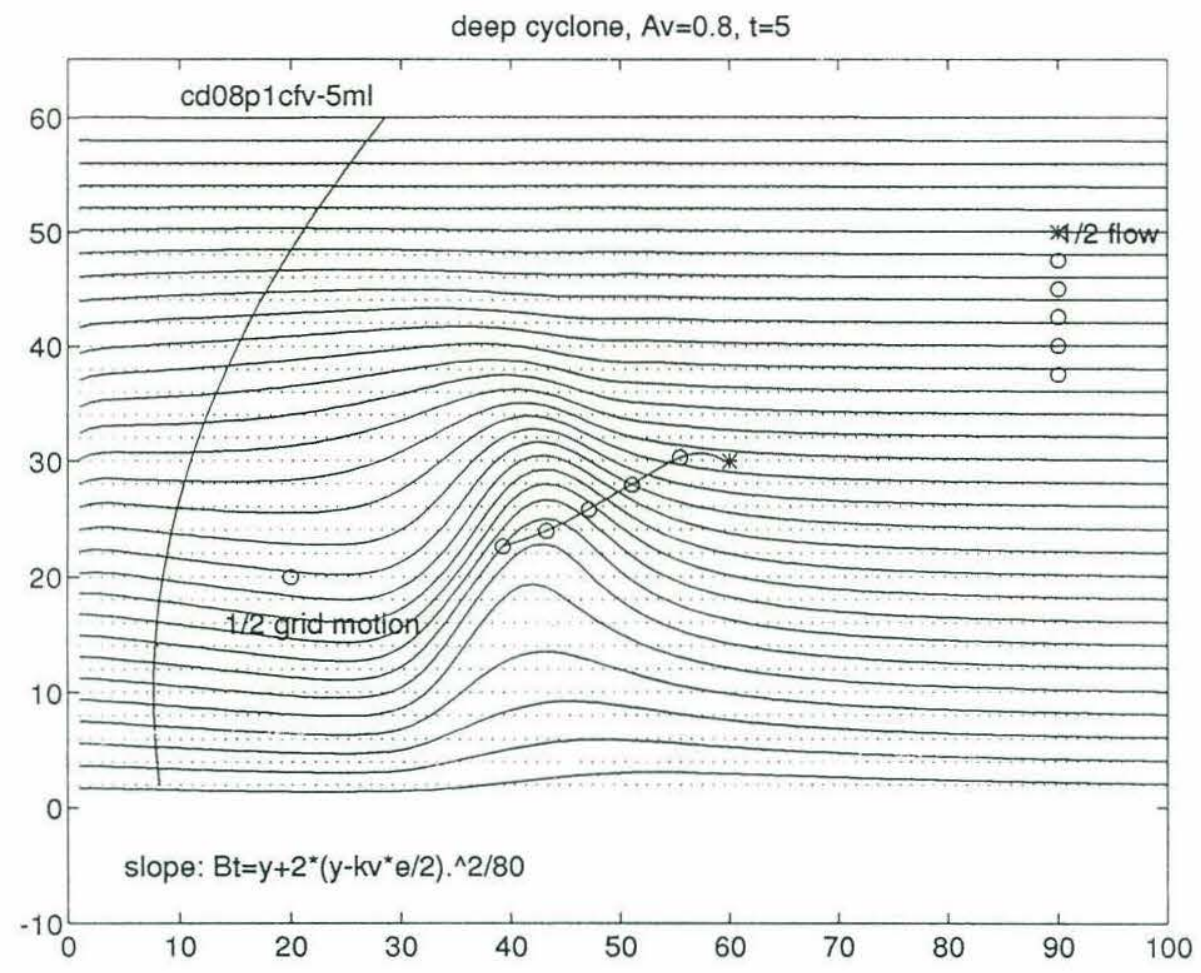

(a)

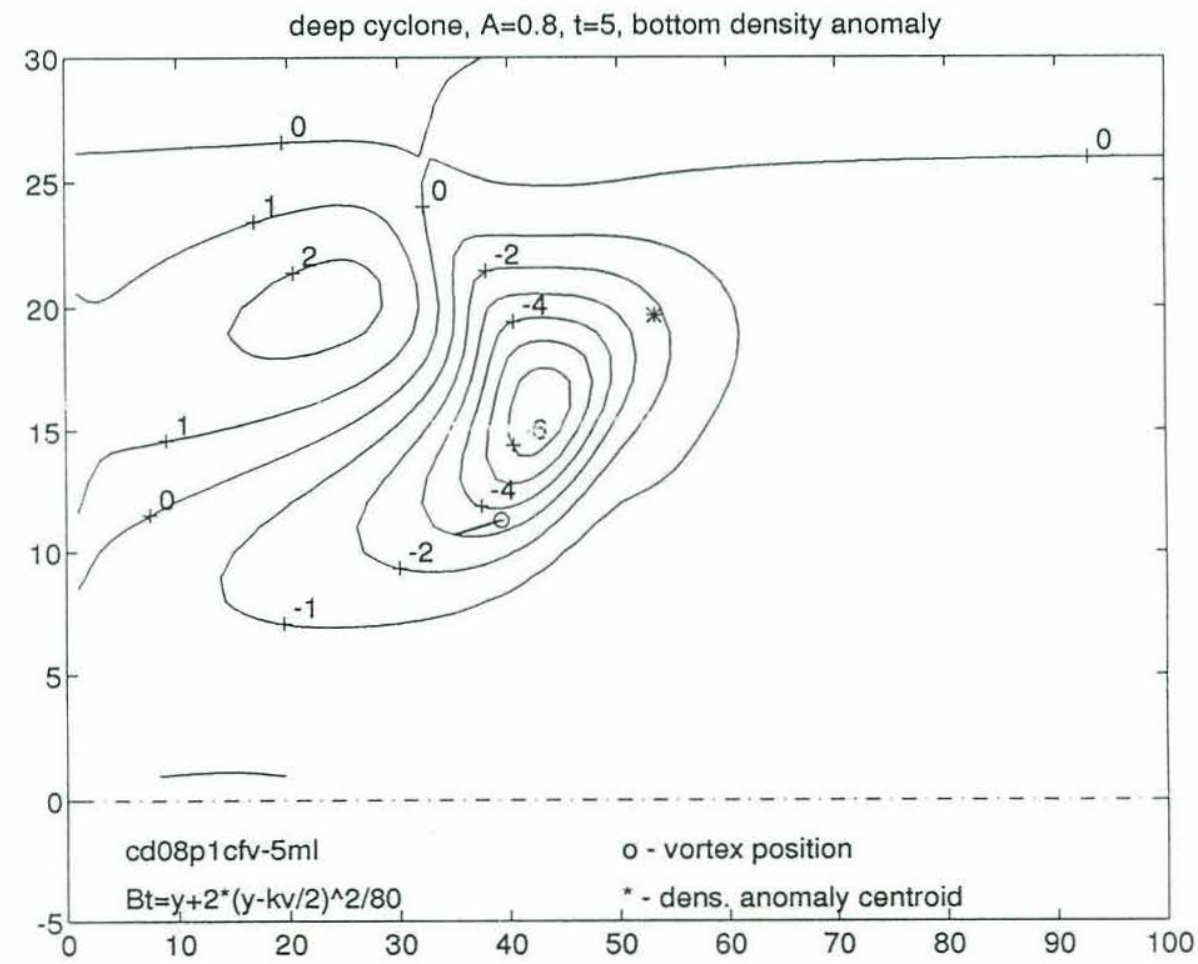

(b)

Figure 6.13 (a,b). Motion of a cyclonic vortex over the slope with positive curvature and the background "southward" flow $\mathrm{v}_{\text {flow }}=-5$. 


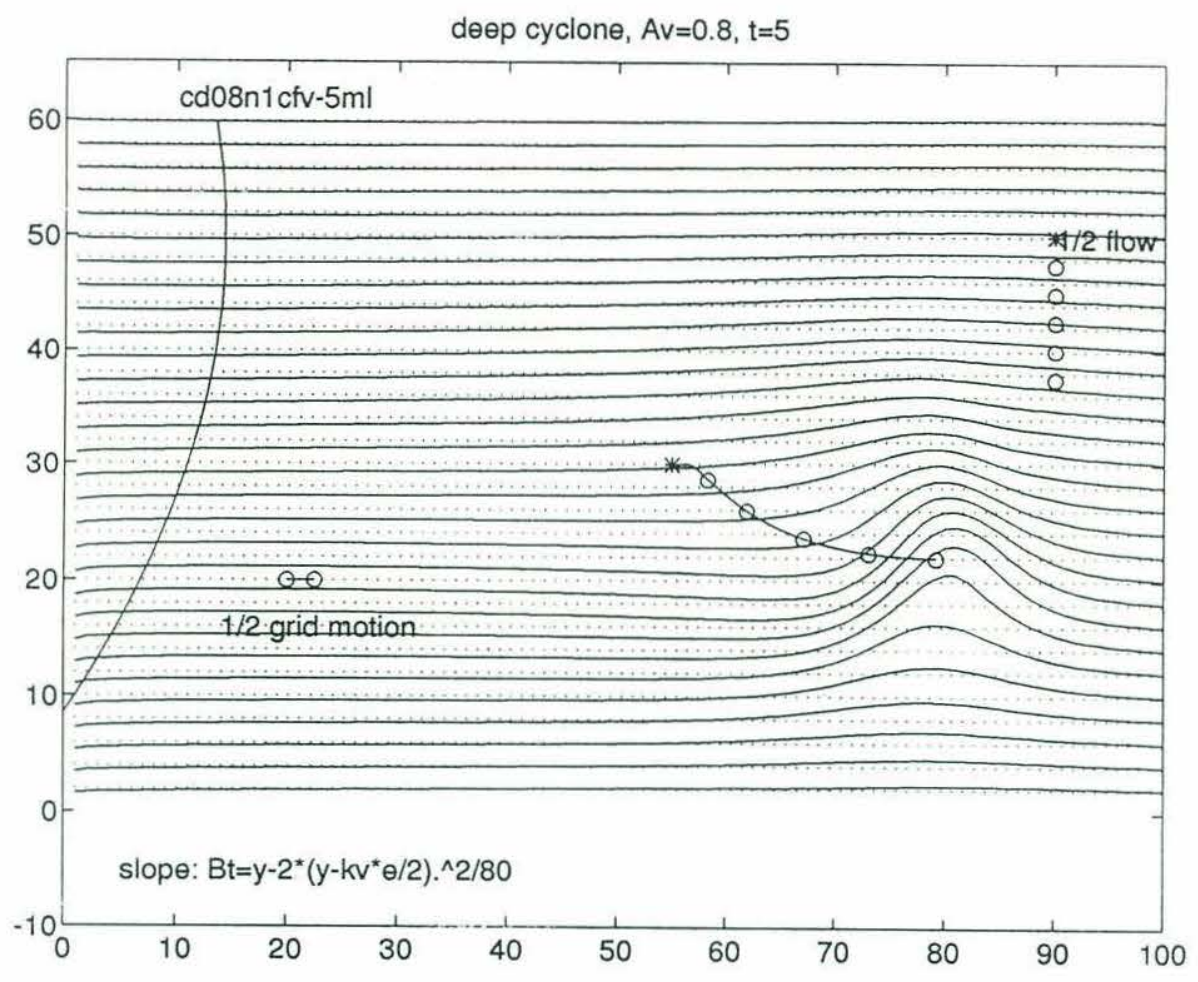

(a)

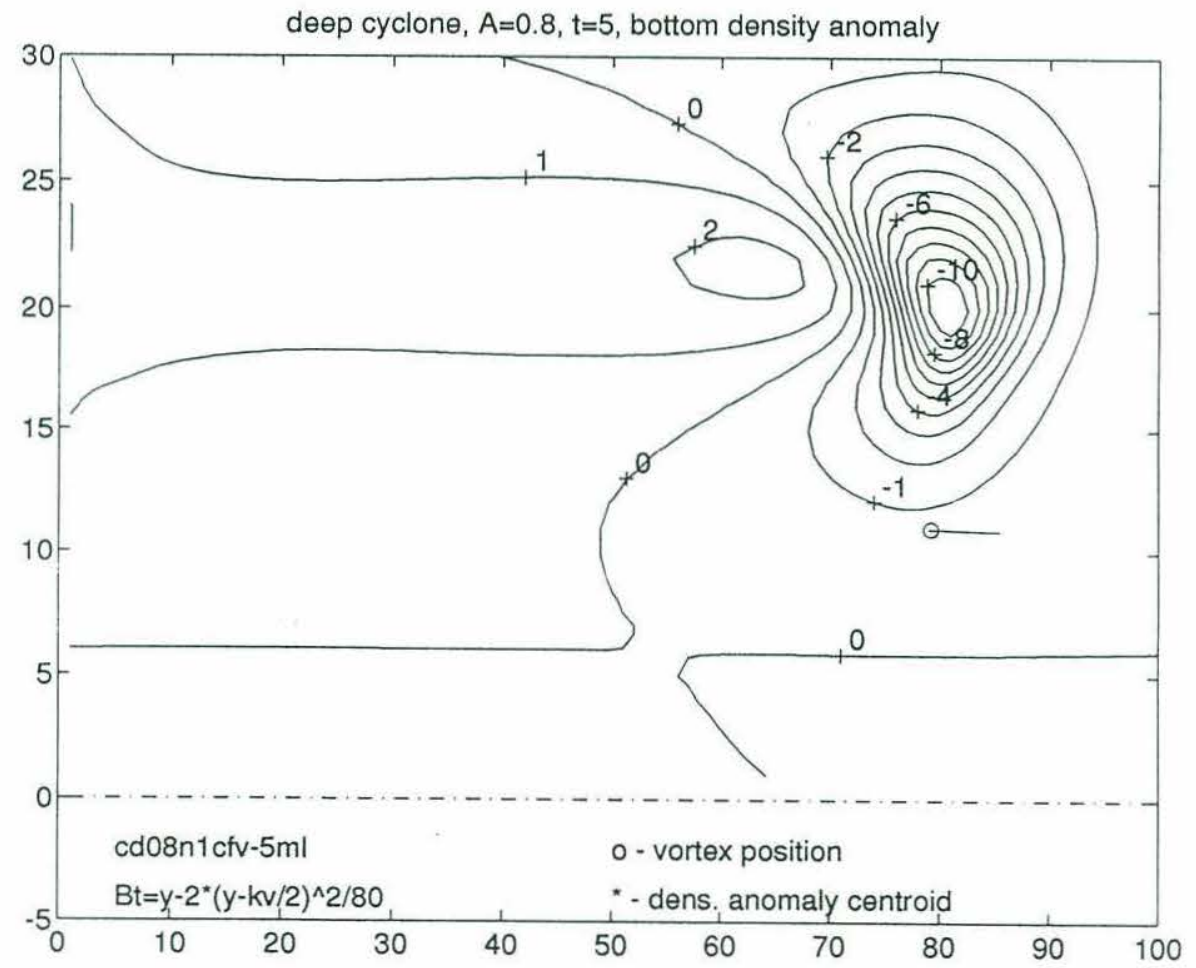

(b)

Figure $6.14(a, b)$. Motion of a cyclonic vortex over the slope with the negative curvature and the background "southward" flow $\mathrm{v}_{\text {flow }}=-5$. 
similar behavior to the case with the surface, although its velocity is inclined slightly "westward" instead of the "eastward" direction. Its speed is just slightly smaller than for the same parameters with the surface. This "westward" component is apparently due to the absence of the surface, because all runs with the constant slope and a finite depth show some "eastward" propagation. Still the actual difference in the direction of the vortex trajectory with and without the surface is only a few degrees and the cross-slope component is dominant in both cases.

Figure 6.10(a,b) shows the trajectory of the same vortex but in the presence of a cross-slope shear flow in the same ("northward") direction as the vortex motion itself. The result is not very different from a simple linear combination of the flow and translational velocity of the vortex in the absence of the flow. Actually the vortex velocity is just slightly stronger than the sum of these two terms, but the difference is only about $10 \%$ which is not significant. The vortex trajectory is exactly cross-slope in this case, without any along-slope component.

When the flow is directed "southward" it counteracts the self-advection tendency so the crossslope velocity component can be significantly reduced or reversed. Runs with such flows are presented in figures 6.11-6.12. The trajectory of the vortex does not differ significantly from the linear combination of its self-propagation velocity and advection by the flow. The density anomaly is much weaker in this case and its circulation does not compensate the strength of a vortex itself. This is not surprising because without the surface there is no barotropic component anyway and there is no necessity to compensate it with the induced vorticity field.

Another example which we would like to demonstrate in this section is a combination of a simple background flow and curvature of the bottom slope. Two similar experiments (the same vortex with $\widehat{\mathrm{A}}=0.8$ and the same "southward" flow with velocity $\mathrm{v}_{\text {flow }}=-5$ ) but with different curvatures of the slope are shown in figures $6.13-6.14(\mathrm{a}, \mathrm{b})$. The flow velocity is again slightly larger and in the opposite direction than the self-propagation velocity of the vortex, so motion is expected to be mainly in the along-slope direction. For the positive curvature (figure 6.13) the vortex velocity is mainly in the along-slope "westward" direction (the same "westward" tendency as without a flow for positive curvature). When the curvature is negative (figure 6.14) the vortex moves "eastward" with approximately the same velocity as in the opposite direction in the previous case.

Summarizing this section we shall conclude that inclusion of a background surface flow in our highly idealized model has not brought any surprizing new effects. The vortex velocity in a steady regime does not depart far from a linear combination of a flow and a self-advection tendency in an otherwise quiescent environment.

In our model we restricted ourselves to baroclinic flows which do not reach the bottom. It is yet to be examined what effects can be caused by more complicated types of flows, with a barotropic component and horizontal and vertical shear, which can directly affect not only a vortex itself but also bottom density contours. These effects are beyond the scope of our present study, although the 
model can be generalized to give background flows a more comprehensive treatment, which is left out for future work.

\subsection{Non-uniform ambient stratification}

In all previous experiments we used a rather strong assumption about a constant buoyancy frequency of ambient fluid. Actual stratification in the ocean changes dramatically and over more than almost one order of magnitude, so the constant $\mathrm{N}$ approximation requires some analysis to be justified.

In our method influence of a variable stratification will change the Green's function which is used in the inversion of PV and bottom density distribution for a velocity field.

In the Appendix 1 I consider a simplified approach to treat a problem with variable stratification. I derive a class of stratification profiles which allows after some manipulations to reduce the differential operator connecting potential vorticity and streamfunction to the same 3dimensional Laplacian as for the case of a constant buoyancy frequency. I show some examples of azimuthal velocity profile for this "parametric" stratification (figure A1.5 a,b). For the surface -intensified stratification profiles, characteristic of the real ocean, most of the difference between velocities for "parametric" and constant $\mathrm{N}$ cases are at the surface from a PV anomaly which is located also near the surface (the second plot in figure A1.5b). For a bottom velocity from a surface PV anomaly or for a surface velocity from a bottom PV or density anomaly this difference does not exceed $\sim 10 \%$ even for the case of $\mathrm{N}_{\text {surface }} / \mathrm{N}_{\text {bottom }}=30$ while qualitatively it looks very similar (the second plot in figure A1.5a and the first one in figure A1.5b). The difference in a surface velocity from a surface vortex will affect internal dynamics of a vortex or its interaction with other surface eddies. For our purpose of the bottom-surface interaction even a large ratio of surface to bottom buoyancy frequencies apparently does not influence velocities very strongly. Thus for our purposes the constant $\mathrm{N}$ assumption is probably not so bad as for a problem in the upper ocean dynamics, as long as we represent a "stretched" depth (which will depend on the stratification profile) correctly.

Still the small difference that is unaccounted for in the constant $\mathrm{N}$ approximation can make some changes in the solution. For the case of surface-intensified stratification velocities of surfacebottom coupling are slightly larger which make the vortex appear "stronger" in comparison with a constant $\mathrm{N}$ ocean. On the other hand the topographic wave frequency is also somewhat greater for 
the equivalent depth in the case of buoyancy frequency increasing toward the surface (figure A1.5a). This implies that the nondimensional amplitude parameter $\widehat{A}$, relevant for the wave-breaking tendency, will remain practically the same as for constant $\mathrm{N}$ and behavior of a vortex will not change significantly.

To verify this I performed several runs in which we tried to simulate a velocity profile obtained for "parametric" stratification. One such experiment is shown in figure 6.15(a,b). Azimuthal velocities used in the inversion procedure (convolution with a corresponding Green's function) were numerically approximated for a close fit to the "parametric" ones. As expected this did not introduce significant differences. The vortex moved "northward" with some "eastward" component with approximately the same speed as in the case of constant $\mathrm{N}$ in previous runs. Its "eastward" velocity was just slightly larger than for uniform stratification which made its trajectory more inclined alongslope, but the difference in angle was only a few degrees. The density anomaly was more elongated and its maximum was shifted somewhat upslope, but again no new qualitative effects were in evidence.

Our "parametric" stratification which we used have a simple monotonic structure and mimic only gross shape of real oceanic profiles. Still I believe that finer details and sharp maximums of real oceanic buoyancy frequency can have significant changes in the velocity field only for interactions of water parcels with small vertical separation. For the surface-bottom interaction, which is the problem in the present study, only this gross structure is significant and, as our example shows, even this does not affect the results strongly enough. The velocity profile from the constant $\mathrm{N}$ case still resembles the actual one more accurately than, for example, the profile from a 2-layer model. In the next chapter we shall consider these differences between the continuously stratified and 2-layer cases and possible consequences which they can make in the behavior of vortices in different models.

\subsection{Finite-volume vortex}

Another assumption which we used throughout the described calculations is that our vortex is a singular PV anomaly placed at the surface (or in the interior of an ocean). Real oceanic vortices have a typical horizontal size considerably larger than the deformation radius and the point vortex approximation appears to be a questionable one. 
On the other hand because a vortex and the bottom are separated by a whole depth of the ocean certainly only integral parameters are important for vortex-topography interaction, since the influence of smaller scales decreases rapidly over a depth.

In this section we present only one experiment in which vortex internal structure has some additional degrees of freedom. Instead of a 3-dimensional delta-function of a PV anomaly it is represented by an infinitely thin patch of uniform PV distribution at the surface bounded by a material contour. Its internal evolution is computed by the ordinary contour dynamics technique, with some special provision for volume conservation. Its interaction with a bottom density field is simplified for computational efficiency. The streamfunction from a vortex is expanded into the multipolar moments (up to the second-order, quadrupolar terms). The velocity field from a bottom density anomaly is also expanded around a vortex in Taylor series and truncated after quadratic terms. This procedure is accurate enough when the radius of a vortex is not much larger than the "stretched" depth.

Motion of such a vortex is shown in figure $6.16(a, b)$. Initially a vortex had a circular shape with the radius equal to two deformation radii. Its bounding contour is indicated by the solid line superimposed on the bottom density contours as in other plots. Another solid line shows its centroid trajectory. One can see that the influence of the desingularization is not very big: its motion resembles that of the point vortex quite closely. The speed of the vortex decreases slightly while the direction remains practically the same. Its "footprint" in the bottom density anomaly is somewhat larger in size which is consistent with some "spreading" of the velocity field due to the finite dimension of the vortex. The shape of the vortex changes slightly as it interacts with the density field at the bottom and the trajectory is slightly "smoother", with smaller wiggles during the adjustment process. But still all these differences are not of principal importance and no new qualitative features appear in this run.

The described approach represents a very simplified treatment of a vortex internal structure and its interaction with the bottom. In particular it does not include the influence of a vertical shear induced in this interaction, because a vortex is represented by a thin patch of PV. In other circumstances, for example when the horizontal size is much larger than the "stretched" depth and a vortex has more complicated internal structure there can be more significant changes in its behavior in comparison with the point vortex model. Yet in our opinion for many oceanic vortices on the continental slopes (but far from the shelf area) their interaction with the bottom can be roughly described within this singular approximation and main results of our study concerning motion of such vortices can be applicable. 
surface cyclone, $\mathrm{Av}=0.8, \mathrm{t}=5$

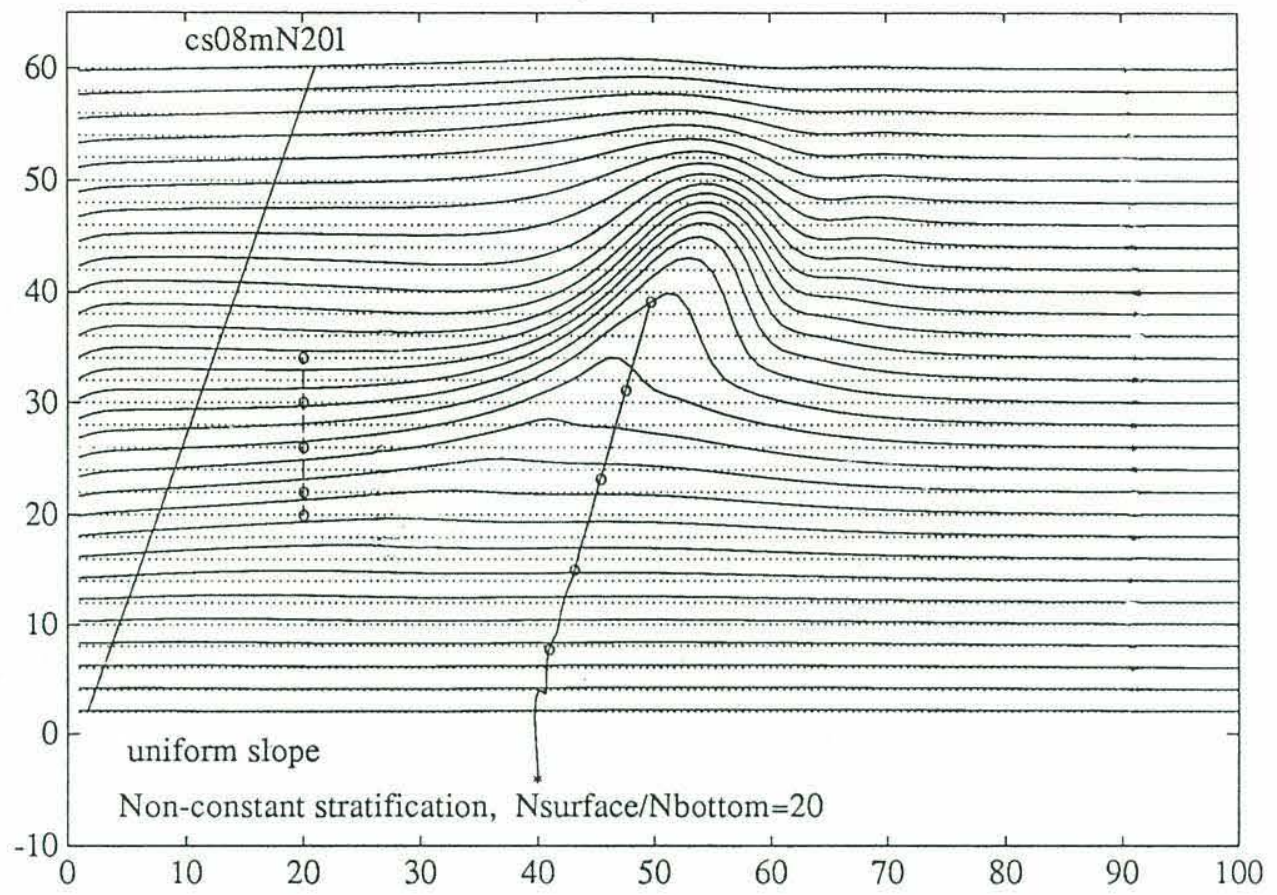

(a)

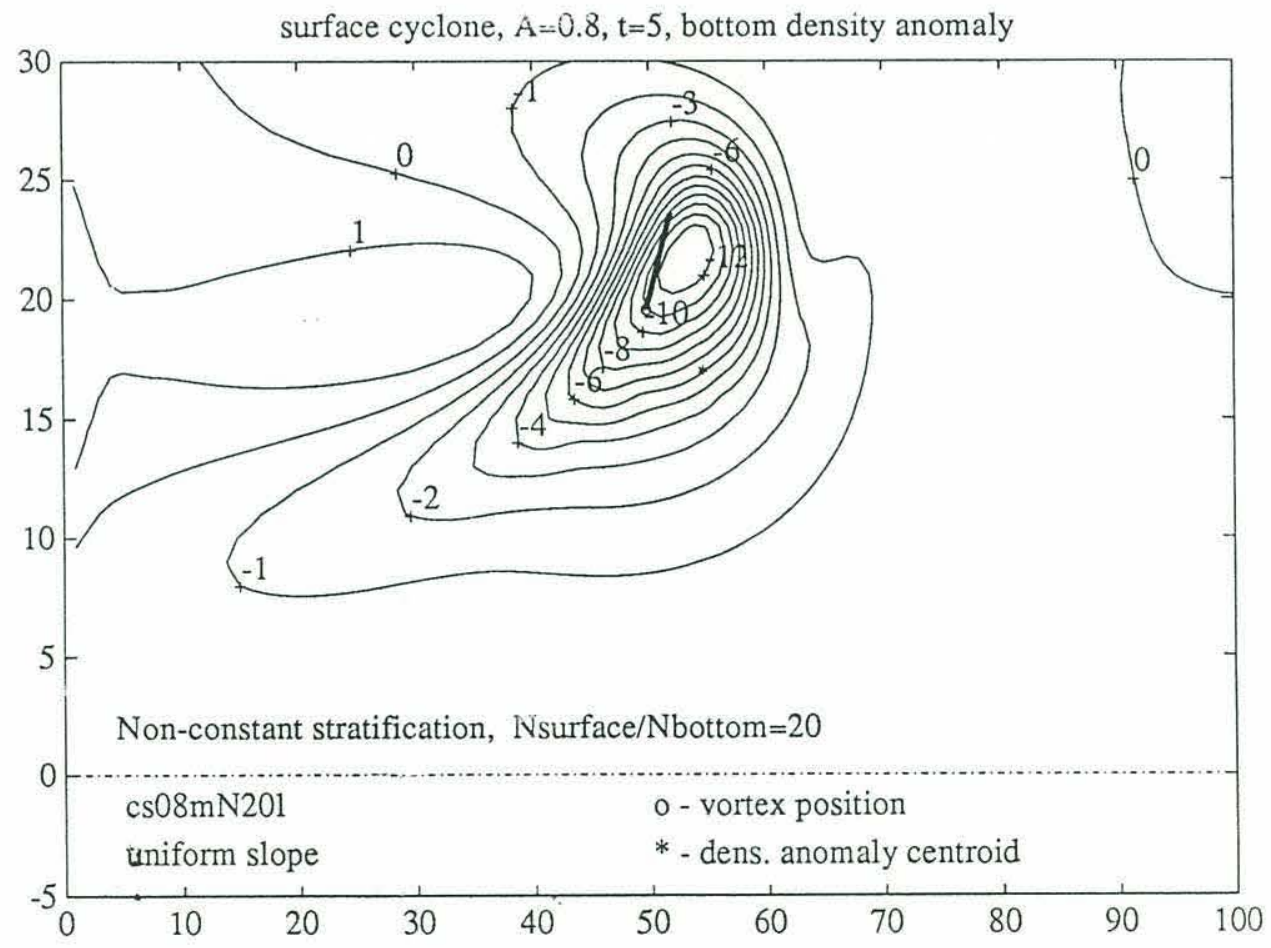

(b)

Figure $6.15(a, b)$. Motion of a cyclonic vortex over the uniform slope and with the "parametric" stratification profile $\mathrm{N}_{\text {surface }} / \mathrm{N}_{\text {bottom }}=20$. 
surface cyclone, $\mathrm{Av}=0.8, \mathrm{t}=5$

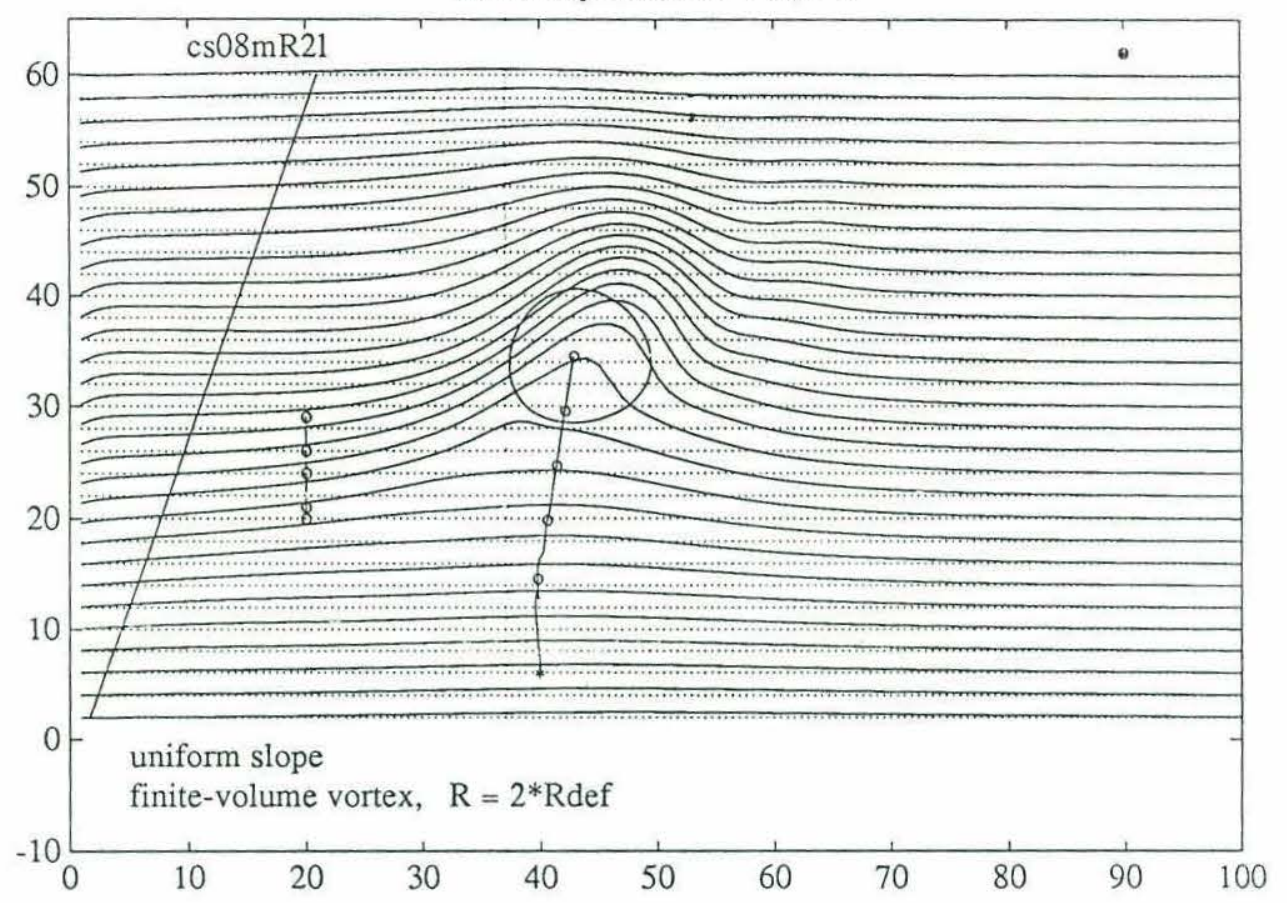

(a)

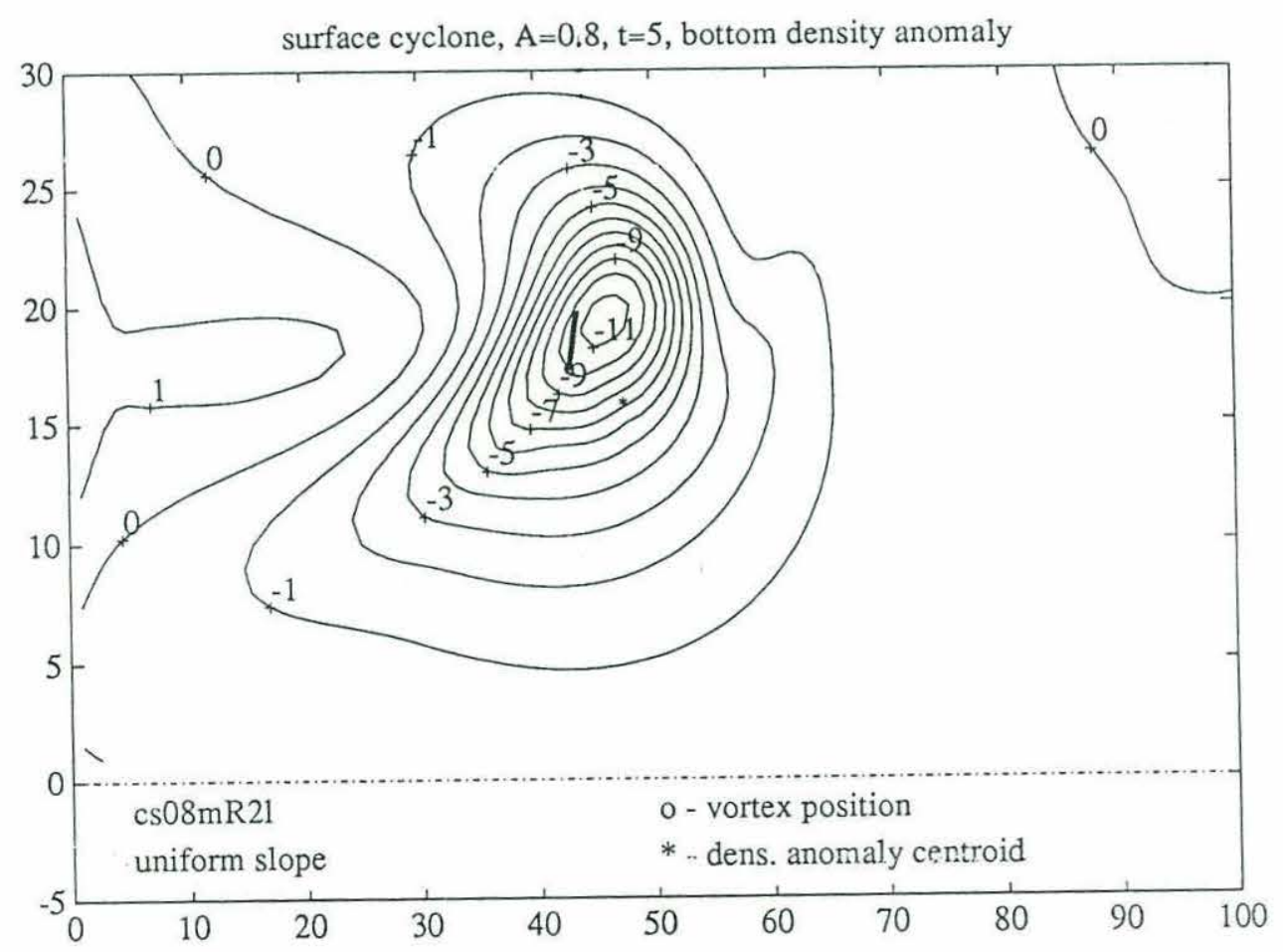

(b)

Figure 6.16 (a,b). Motion of a finite-volume cyclonic vortex with the radius $R=2 R_{\text {def }}$ over the uniform slope. 
In this chapter I considered a variety of factors which can influence the vortex-topography interaction. I studied only a few possibilities and a small fraction of a parameter range and often in a crude and simplified manner. Therefore my results have certainly limited validity and applicability. Most of the attention was given to such factors as variations and irregularities in bottom topography and presence of background surface flows. None of these contributions seem to change the most important observed tendency of cross-slope motion of vortices, "northward" for cyclones and "southward" for anticyclonic ones. But still the presence of additional effects can evidently change some features of vortex behavior, especially its along-slope motion.

Most important in my opinion among the factors studied are variations in slope angle and small-scale topographic irregularities. These perturbations even of small amplitude are able to change significantly the direction of vortex motion. Background flows which act directly only upon a vortex itself do not seem to have as much influence on its behavior: the vortex velocity does not depart significantly from a simple sum of its self-propagating velocity and flow advection. Influence of more complicated external flows, especially unsteady and with a non-uniform potential vorticity structure, remains to be studied.

Non-constant ambient stratification and internal dimensions of a vortex also appear to have limited influence on its propagation. One can certainly imagine a situation when a large vortex with more complicated internal structure and embedded into strongly variable stratification profile would behave differently from the presented results. But this again remains beyond the scope of the present study. 


\section{Chapter 7}

\section{Planetary vs. topographic}

\section{beta-effect}

\subsection{Initial evolution: similarities}

In this chapter we shall discuss and compare qualitatively some characteristics of vortex motion on the planetary $\beta$-plane on the one hand and over a uniform topographic slope on the other. In the barotropic quasigeostrophic dynamics these two cases are identical with a topographic parameter

$\beta_{t}=\alpha f / H \quad(\alpha-$ slope angle, $H$ - reference depth of an ocean, $f$ - coriolis parameter which is assumed constant). The results of the two previous chapters with the continuously stratified model indicate that there are clearly some similarities as well as differences between these two situations. Moreover these similarities and differences can be tracked down to the particular stages of vortex evolution on the $\beta$-plane and over a topographic slope. While the initial motion of a vortex is very similar in both of these situations, the later stages differ significantly in these two cases.

In both cases if started initially from an unperturbed environment a vortex moves rapidly along the gradient of an active tracer field. This coincides with the meridional direction on the planetary $\beta$-plane and the cross-slope one in the topographic case. This process can be easily understood in terms of the interaction of a vortex with a surrounding vorticity field induced by it. For simplicity we shall consider qualitatively only a very short initial stage of this motion. By the "initial stage" we imply two main assumptions. First - a secondary field induced by a vortex when it perturbs background vorticity or density fields is still weak and we can neglect its self-interaction. Second - the vortex displacement from its initial position remains very small so that its velocity field can be considered stationary and radially symmetric. This initial stage can last actually only a fraction of a characteristic period of corresponding Rossby waves. 
The vortex interaction with the background field in this initial stage can be qualitatively described as following. The velocity field from a vortex perturbs isolines of an active tracer antisymmetrically around itself. This induces a secondary vorticity field having a dipolar structure aligned along the unperturbed isolines. Because of geostrophy this secondary field pushes a vortex "meridionally" - along the tracer's gradient. This is shown essentially in the figure 6.6 for the topographic case. Cyclonic vortices will move northward on the $\beta$-plane and upslope over topography and anticyclones - southward and downslope. Because velocity induced by a vortex is constant initially, the displacement of tracer isolines will grow linearly in time. This means that the initial dipolar structure will be almost stationary in space and growing linearly in time and the velocity of the vortex itself will also grow linearly in time.

This initial evolution of an isolated vortex on the planetary $\beta$-plane was studied extensively because of its important meteorological application (motion of strong tropical cyclones). One can refer to some analytical works (for example Reznik, 1991, for a point-vortex approximation and the recent paper of Sutyrin and Flierl, 1993, for a more complicated vortex structure) and numerical studies (for example McWilliams and Flierl, 1979) and in other related works. In all these studies initial meridional motion with a constant acceleration was found (in some cases there was also a nonzero zonal initial velocity).

If we compute this initial meridional acceleration of a vortex (not necessarily a point one) with a non-zero barotropic circulation in the horizontally unbounded domain with the rigid-lid approximation the result will surprisingly give us infinity for both the planetary $\beta$-plane and a topographic slope. This was the reason to use either a finite deformation radius approximation (like in Reznik, 1991) or spherical geometry (Bogomolov, 1985, Sutyrin, 1988) in these analytical studies. But this singularity in the acceleration only underlines the inconsistency of the infinite $\beta$ plane or an infinite topographic slope approximations: both of them can not exist in reality. For a numerical model this does not represent a major problem. Although this initial acceleration can be strong if the vorticity field is integrated numerically over a large domain (as in our model), this initial stage lasts only a brief moment and does not influence significantly the subsequent evolution.

After this short period of meridional acceleration the nonlinear evolution of the induced vorticity field and vortex motion itself become important. These nonlinear processes induce some along-gradient asymmetries and, correspondingly, another dipolar structure aligned in the perpendicular direction to the first one. This results in the growing "zonal" component of the vortex velocity - westward for the planetary $\beta$-plane and to the left of the upslope direction for the topographic case. These results are similar for many numerical studies of vortex dynamics, for example McWilliams and Flierl (1979) and other works. The trajectory of a vortex turns slightly "westward" in both cases, showing again the similarity of the planetary $\beta$-plane and a topographic slope. But after that, at a time moment approximately equal to the characteristic wave period, the 
behavior of a vortex in these two situations diverges as the nonlinearity of the secondary vorticity field increases. One possible reason for this divergence will be discussed in the next section.

\subsection{Differences: "wave-gliding" vs. "wave-breaking" regimes}

The evolution of a localized oceanic vortex on the planetary $\beta$-plane has been a subject of numerous studies. In the context of long-period numerical experiments with oceanic vortices one can mention McWilliams and Flierl (1979), Mied and Lindemann (1979) and many other subsequent works. These studies showed near-steady westward translation (although completely steady motion was never achieved) with some meridional motion; a northward for cyclones and a southward for anticyclonic eddies.

Similarities between the planetary $\beta$-plane and a topographic slope in the initial stage can be easily understood and was discussed in the previous section. Both a meridional displacement of fluid parcels in the planetary case and cross-slope motion of bottom density contours over a slope in the stratified topographic case produce vorticity anomalies in a similar fashion. This vorticity production is the cause of planetary and topographic Rossby waves, which are qualitatively similar to each other. But in the later, fully nonlinear stage of the vortex motion this simple analogy is not sufficient and more complicated dynamical balances must be taken into account. In my opinion the most important process determining the long-term behavior of vortices in these two situations is the interaction between the vortex-induced swirl motion of surrounding fluid parcels and radiation of waves away from a vortex. For simplicity I shall call it the breaking-radiation balance. When a vortex is weak enough waves induced by its velocity field will quickly radiate away. In the case of a strong vortex its swirl velocity can break these waves and water parcels will be rotating around it in near-circular trajectories. Isolines of initially unperturbed potential vorticity field then will be twisted around a vortex. It is not surprising that the behavior of a vortex will be very different in these two dynamical regimes. I believe that this difference is responsible for the discrepancy between our results with a topographic slope and simulation of vortices on the planetary $\beta$-plane.

We estimate that typically for upper-ocean eddies over a topographic slope on the one hand and in the presence of the beta-effect in the flat-bottom ocean on the other hand the situation will be different with respect to this wave-breaking and wave-radiating tendencies.

In the case of the strong vortices on the $\beta$-plane their potential vorticity contrast over a characteristic lengthscale is likely to be much greater than its planetary gradient. In other words the 
parcel swirl velocities are much greater than the phase velocities of Rossby waves. A typical barotropic or baroclinic Rossby wave velocity of the lengthscales not much larger than the deformation radius is about $5 \mathrm{~m} / \mathrm{sec}$ while the swirl velocity in the vicinity of strong surface vortices can easily reach $50 \mathrm{~cm} / \mathrm{sec}$. This implies that not only these waves are highly non-linear but they are constantly broken by a vortex and the planetary PV gradient is vigorously eroded as water parcels are "stirred" around a vortex. The persistence of this wave-breaking process is actually a "survivability" condition for oceanic eddies. Because such vortices are embedded into the planetary vorticity gradient they can lose their identity by mixing with surrounding water if they are not strong enough. So that when they are not highly nonlinear initially they will quickly disperse into a packet of Rossby waves (one can refer to Flierl, 1977, for the details of this process).

Therefore strong vortices on the planetary $\beta$-plane must break Rossby waves around themselves and twist (initially unperturbed) isolines of planetary vorticity into highly convoluted spiral structures. Several stages of this process are sketched in figure 7.1. Vortex motion in the later stage is determined by its interaction with surrounding vorticity field. It can approximately be represented by the two dipolar structures which we already mentioned in the previous section. One of them is aligned in the north-south direction and is produced due to the influence of surrounding trapped water parcels which rotate around a vortex in almost circular trajectories. Because of the exchange of vorticity between the "active" and planetary parts parcels on the northern side of a vortex will have the excessive anticyclonic "active" vorticity and, correspondingly, the excessive cyclonic vorticity when they are in the southern half-circle. The resulting dipolar structure advects a vortex westward. This is a well-known mechanism for the vortex translation on the $\beta$-plane. There is also another, east-west dipolar structure which is associated with the unsteadiness of the process. Because in the course of the vortex motion new parcels are constantly involved in the rotating motion around a vortex, their meridional motion on the left and the right side of a rotation center results in the antisymmetric vorticity structure which advects cyclonic vortices northward and anticyclones southward. This east-west dipolar structure is strong initially (its role in the initial meridional acceleration of the vortex was discussed in the previous section) and decreases in magnitude as the vortex reaches almost steady westward translation. We shall refer to this type of motion, depicted schematically in the figure 7.1 as the "wave-breaking regime".

For an upper-ocean vortex over a topographic slope this later stage can be different. The swirl velocity of a baroclinic vortex is typically smaller at the bottom than at the surface and the speed of topographic Rossby waves over the continental slopes is much larger than the speed of planetary waves. While a vortex on the planetary $\beta$-plane has to break planetary Rossby waves around itself to preserve its identity, there is no such necessity in the case of topographic Rossby waves. Moreover, the vortex has to be exceptionally strong or the ocean is shallow enough for this breaking 
Wave - breaking regime:
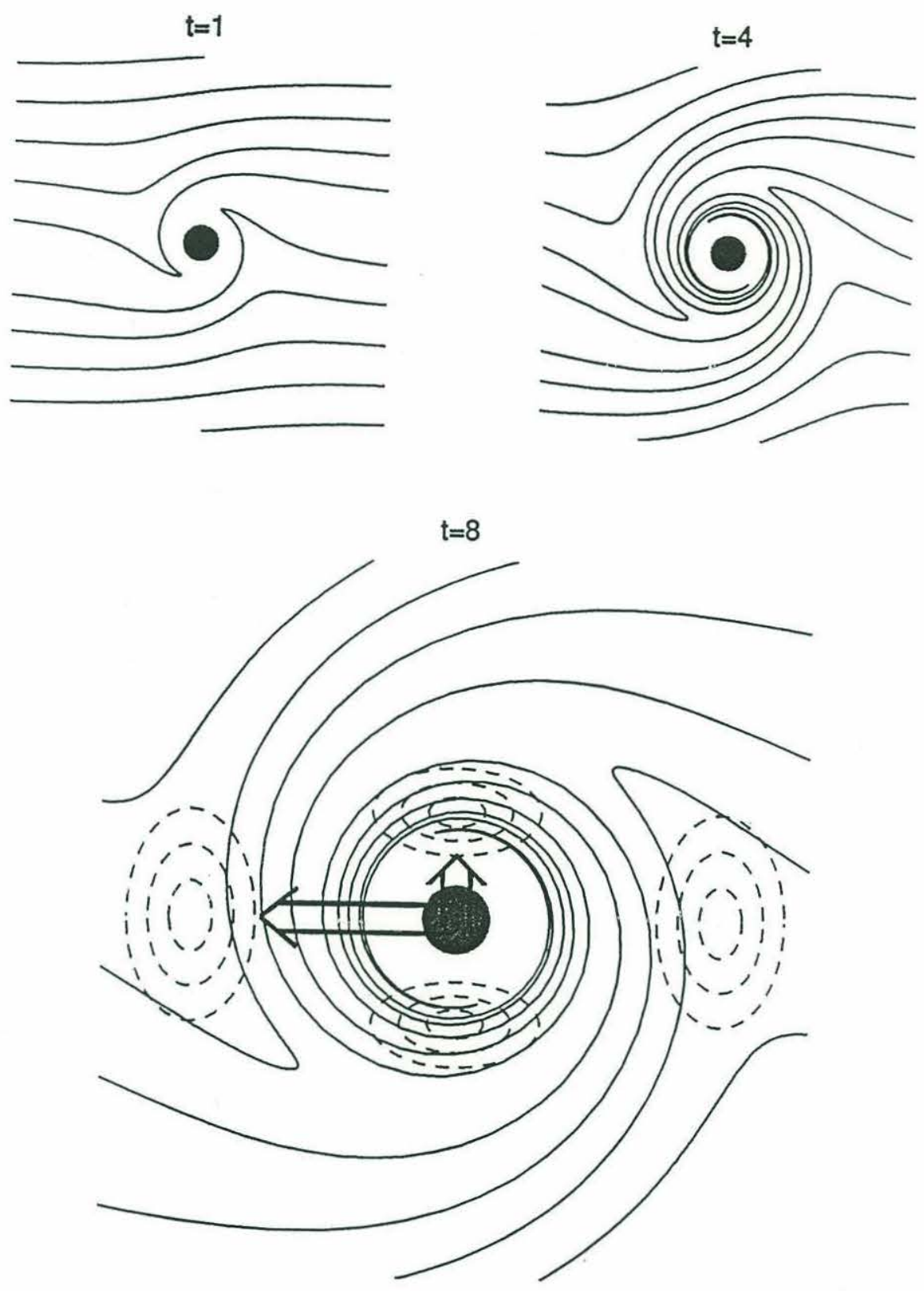

almost westward advection

Figure 7.1. Near-steady vortex motion on the planetary $\beta$-plane: the "wave-breaking" regime. 


\section{Wave - gliding regime:}
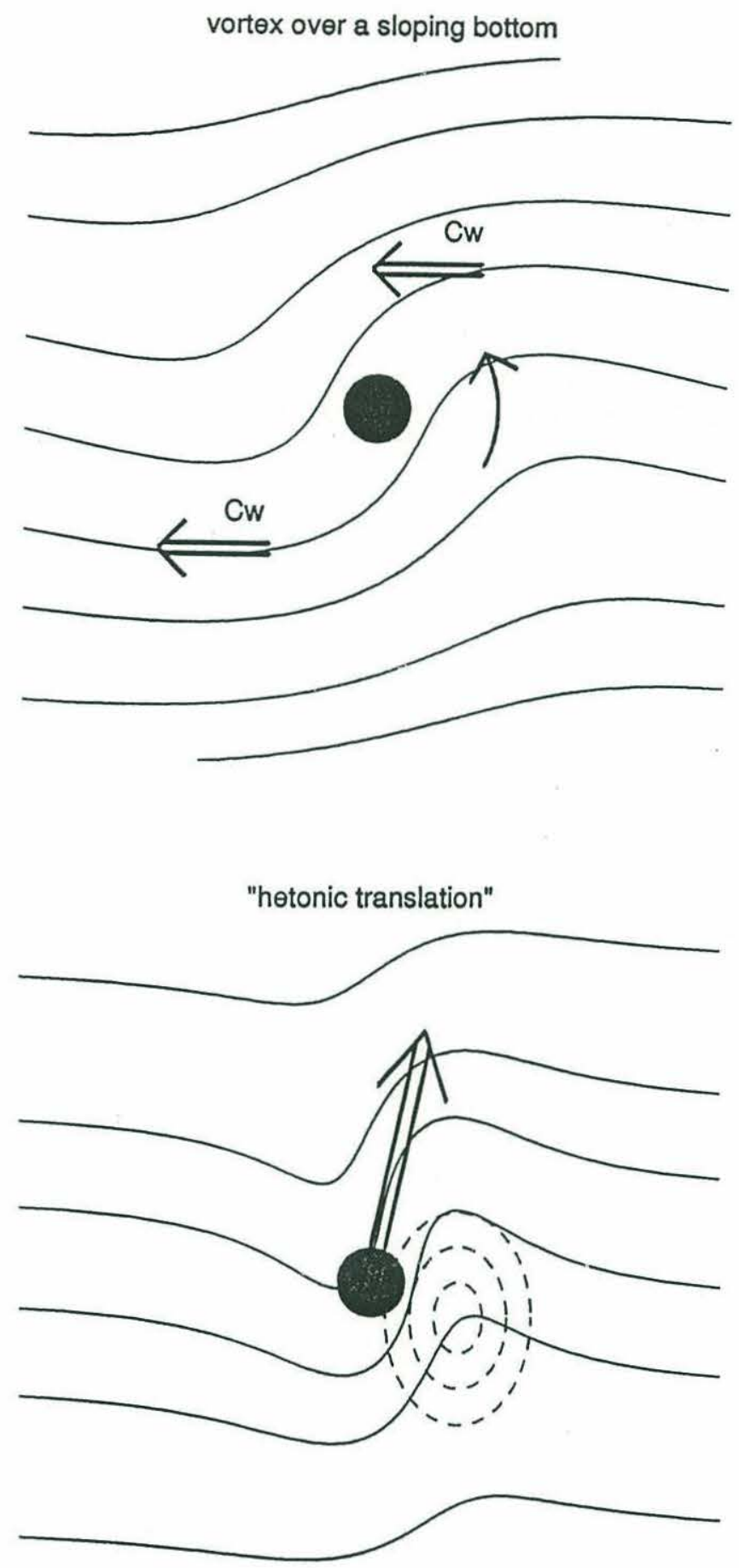

almost upslope (northward) motion

Figure 7.2. Steady vortex motion over a uniform slope: the "wave-gliding" regime. 
to occur. This difference between planetary and topographic waves is facilitated by two circumstances. The first - that an upper-ocean vortex and the bottom are separated vertically and the azimuthal velocity from a vortex is weaker than near the surface and has a much smoother profile (we shall consider this in some details in the next section). The second reason is that the typical phase velocity of topographic waves in the regions of continental slopes with angles $10^{-3}-10^{-2}$ is much larger $(20-100 \mathrm{~cm} / \mathrm{s})$ than than the velocity of planetary waves $(3-10 \mathrm{~cm} / \mathrm{s})$. To stress this difference some authors, for example Rhines (1977), referred to topographic and planetary Rossby waves as "fast" and "slow" baroclinic waves respectively.

This failure of wave-breaking can be responsible for the difference of the vortex behavior in the later stage for the planetary and topographic cases. For a vortex over a slope its initial "east-west" dipolar anomaly is not broken by the velocity field but transformed instead: Its "like-signed" part which was initially situated on the "western" side is dispersed into outgoing waves and the "opposite-signed" part shifts slightly "westward" under a vortex and forms a coupled "hetonic" structure together with a vortex itself. This coupled structure is sketched in figure 7.2. We shall refer to this type of motion as the "wave-gliding" regime to distinguish between the predominantly "westward" motion on the planetary $\beta$-plane.

This difference between the topographic and planetary cases is mainly of a quantitative rather than qualitative nature. When the stratification is very weak and a flow is almost barotropic then the baroclinic "hetonic" structure can fail to materialize and the role of topography will be more similar to that of the planetary $\beta$-plane.

The persistence of the "wave-gliding" regime would not necessarily imply only cross-slope motion. As we have seen in previous chapter, in the presence of nonuniform slope, topographic perturbations and background flows characteristic of the real ocean, the direction of vortex motion can vary strongly and sometimes the along-slope velocity can be dominant. But in this case it is actually harder to predict the direction of along-slope motion: it can be "westward" or "eastward" in different environments in comparison with more definite westward motion for the "wave-breaking" regime on the planetary $\beta$-plane. The arguments discussed above also do not imply that the "wavebreaking" regime can not occur for the vortices over a slope. But in our opinion such occurrence should be limited to very strong vortices in the relatively shallow regions, for example near the continental shelves. For steep continental slopes far from the coastal regions the breaking of topographic waves is unlikely and the "wave-gliding" (essentially baroclinic) mechanism should be dominant for motion of near-surface eddies in these regions. 


\subsection{Comparison of the representation of a vortex in a continuously stratified and a two-layer model.}

Some previous studies of vortex interaction with topography assumed the qualitative similarity between varying bathymetry and the planetary $\beta$-effect. Usually such simulations were carried out with a barotropic or 2-layer model. Although the dynamics is more complicated in the latter case, motion of vortices over topography was usually interpreted using this analogy. Our results suggest significant difference between these two situations and we shall try to explain the reason for this difference here in this section. We shall briefly discuss some properties of continuously stratified and layered QG models. We would not attempt a detailed comparison but instead using a simple example in the light of the discussion of the previous section we shall suggest that these two types of models can produce somewhat different results for a problem of eddy-topography interaction.

Our results are not easily comparable with those in a layered model. While we considered a uniform slope such configuration is more difficult to implement in the layered model, especially in the QG form. Usually (for example O'Brien \& Smith, 1983 and Smith, 1986), topography was represented by a strip of a uniform slope bounded by a flat bottom and vertical wall. The size of the vortex itself was comparable to the width of the slope, while in our case characteristic lengthscale of topography is much larger than deformation radius. Our model is quasigeostropic while in the above mentioned papers primitive equations were used and the planetary $\beta$-effect was also included. The resulting motion of vortices in the 2-layer model was more complicated but nevertheless was interpreted in terms of a combination of the planetary and topographic $\beta$-effects. We proposed a different interpretation in terms of "wave-breaking" and "wave-gliding" regimes which distinguishes these two situations.

In the previous section we argued that for such type of dynamics it is very important to represent the wave-breaking and wave-radiation tendency correctly since they determine the evolution of the vortex in the later stage. In this section we compare this wave-breaking tendency for our continuously stratified and the 2-layer model (both in the QG approximation) in a very simple fashion. We consider azimuthal velocity and angular velocity profiles at the bottom from a typical upper-ocean vortex as it can be represented in these two models. Figure 7.3 shows the sketch of such a vortex in 2-layer and continuously stratified cases. In both models we consider a

horizontally circular eddy with the same circulation and uniform PV distribution and with the radius measured by the deformation radius of the system. For the two-layer model a vortex has a PV anomaly in the upper layer. In the continuously stratified model it is represented by a thin patch of PV placed near the surface (although real vortices have a finite vertical structure as well and this can influence the velocity profile too). But generally details of the vortex structure are not significant for 
the bottom flow. The azimuthal velocity profile from such vortices is easily calculated. The results for both cases are shown in figure 7.4.

Although a general form of these profiles is similar and they naturally tend to the same (barotropic) limit for large distances, there is quite a big quantitative difference in the bottom velocities for moderate values of the radius $r$. Velocity profiles in the two-layer model are much "sharper" and have much bigger maximum values around $\mathrm{r}=1$ (deformation radius). In addition they depend strongly on the size of the vortex (velocity from a point vortex has a singularity even at the bottom). For continuous uniform stratification the velocity profile is much "smoother" and depends on the size of the vortex only slightly. The velocity maxima are considerably smaller and are shifted toward larger values of $\mathrm{r}$.

This comparison is made for vortices with the same barotropic circulation. It is not the only way of comparison of vortices in different models. One can use, for example vortices with the same surface or upper layer velocities and the results of such comparison can be different. But still a twolayer vortex has a velocity profile with "sharper" gradient, which helps the wave-breaking tendency.

These profiles are computed for the case of $h_{1} / h_{2}=0.3$ and $h_{v}=0.9$ for the two-layer and continuous stratification respectively. This ratio is not however optimized for topography in the sense of the "calibration" recipies of Flierl (1978). But in both cases velocities do not depend strongly on the vertical size of the vortex (layer thickness ratio in the two-layer model and a vortex relative height $h_{V}$ for continuous stratification), as long as the upper layer is thinner or a vortex is near the surface.

The corresponding angular velocity $\mathrm{v} / \mathrm{r}$ under a vortex is much bigger for the 2-layer model. For a vortex radius twice smaller than the deformation radius its maximum is almost four times as big as it is for continuous stratification, for a vortex size equal to the deformation radius it is about two and a half times bigger. In addition for the two layer case it varies strongly over a distance of one deformation radius which implies the strong horizontal shearing of the flow even in the lower layer.

This simple picture suggests that the "breaking-radiation" balance can be represented very differently in these two models. The probability of the "wave-breaking" regime can be much higher in the layered case because of bigger angular velocity induced by the vortex. Although strong oceanic eddies have a typical size considerably larger than the deformation radius, the region of the highest potential vorticity anomaly as it is represented in the numerical models can be much smaller which will result in the unrealistically large angular velocity for small distances from the vortex center. Therefore it is more likely for a vortex in a 2-layer model fall into the "wave-breaking" regime, while continuous stratification can change its behavior (in a model) significantly.

For the continuously stratified case we used an assumption of a constant buoyancy frequency profile for simplicity of calculations. But as we show later in Appendix 1 and was already 
discussed in section 6.3 even a strongly variable buoyancy frequency has a limited influence on the velocity profile at the bottom. The constant $\mathrm{N}$ approximation is still more realistic than the singular delta-function buoyancy frequency profile of a layered model.

This simple example shows that for a two-layer model it is more likely for a vortex to behave similarly to the planetary $\beta$-plane case because of the higher probability of wave-breaking. We argue that a continuously stratified model behaves more realistically in this sense, which was one of the reason to adopt it in our numerical experiments. We would not claim however that the twolayered model is wrong, but there are some features of it which can lead to considerably different representations of important dynamical balances. 

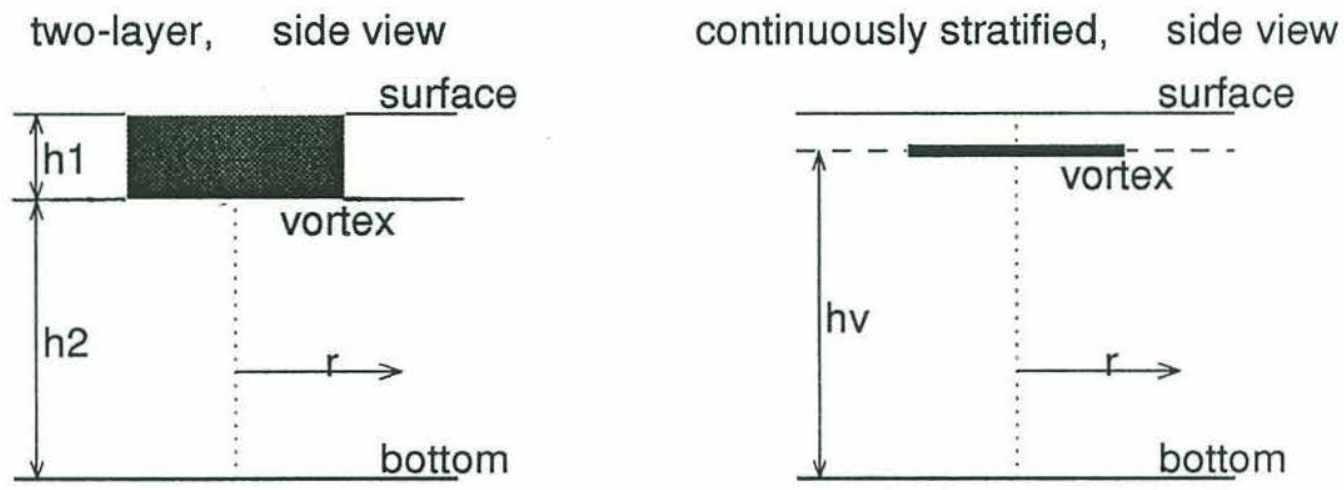

two-layer, top view

continuously stratified, top view
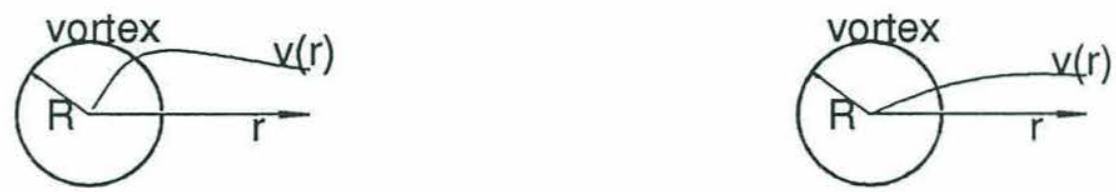

Figure 7.3. Sketch of a vortex for the two-layer and the continuously stratified models. 


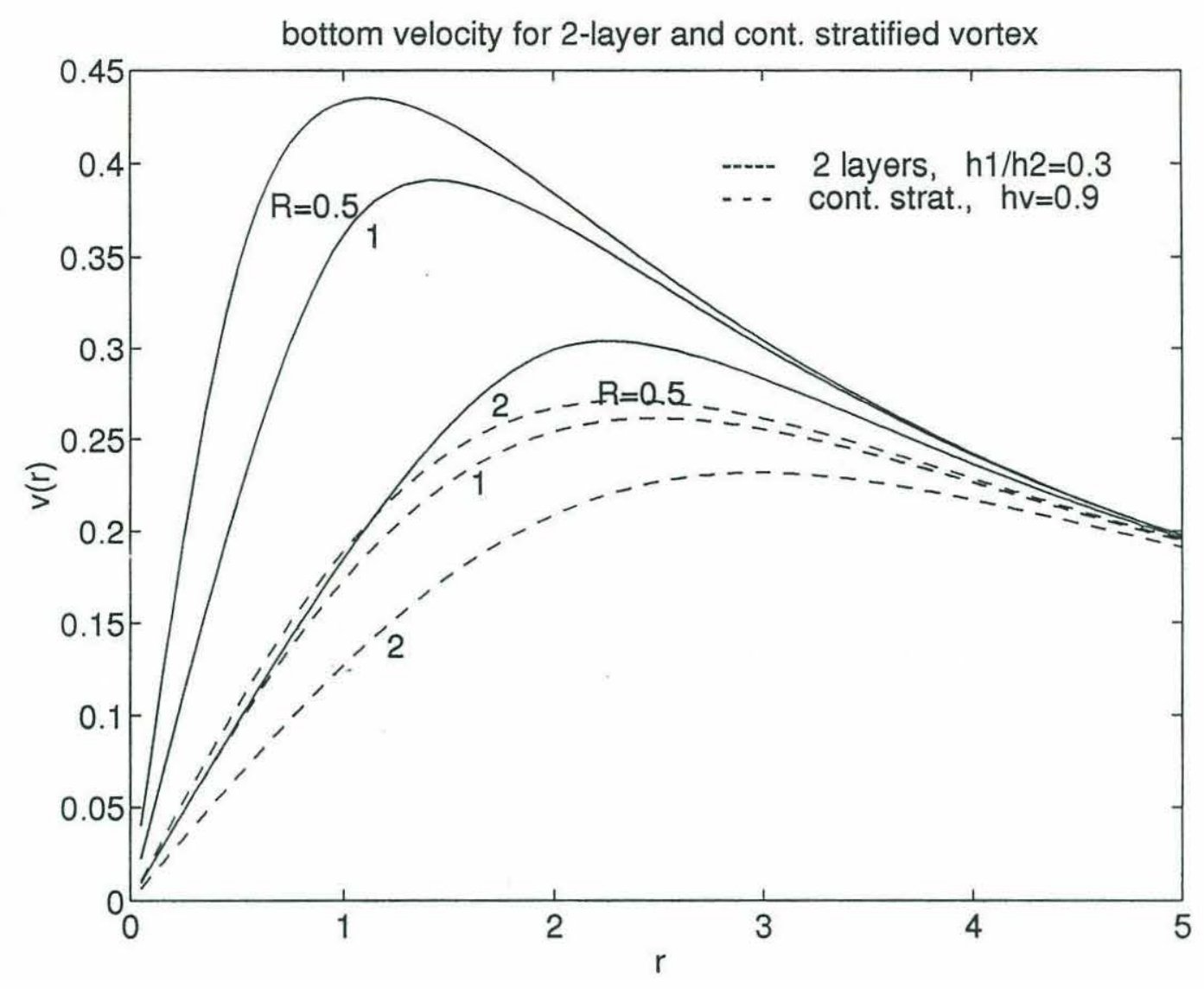

(a)

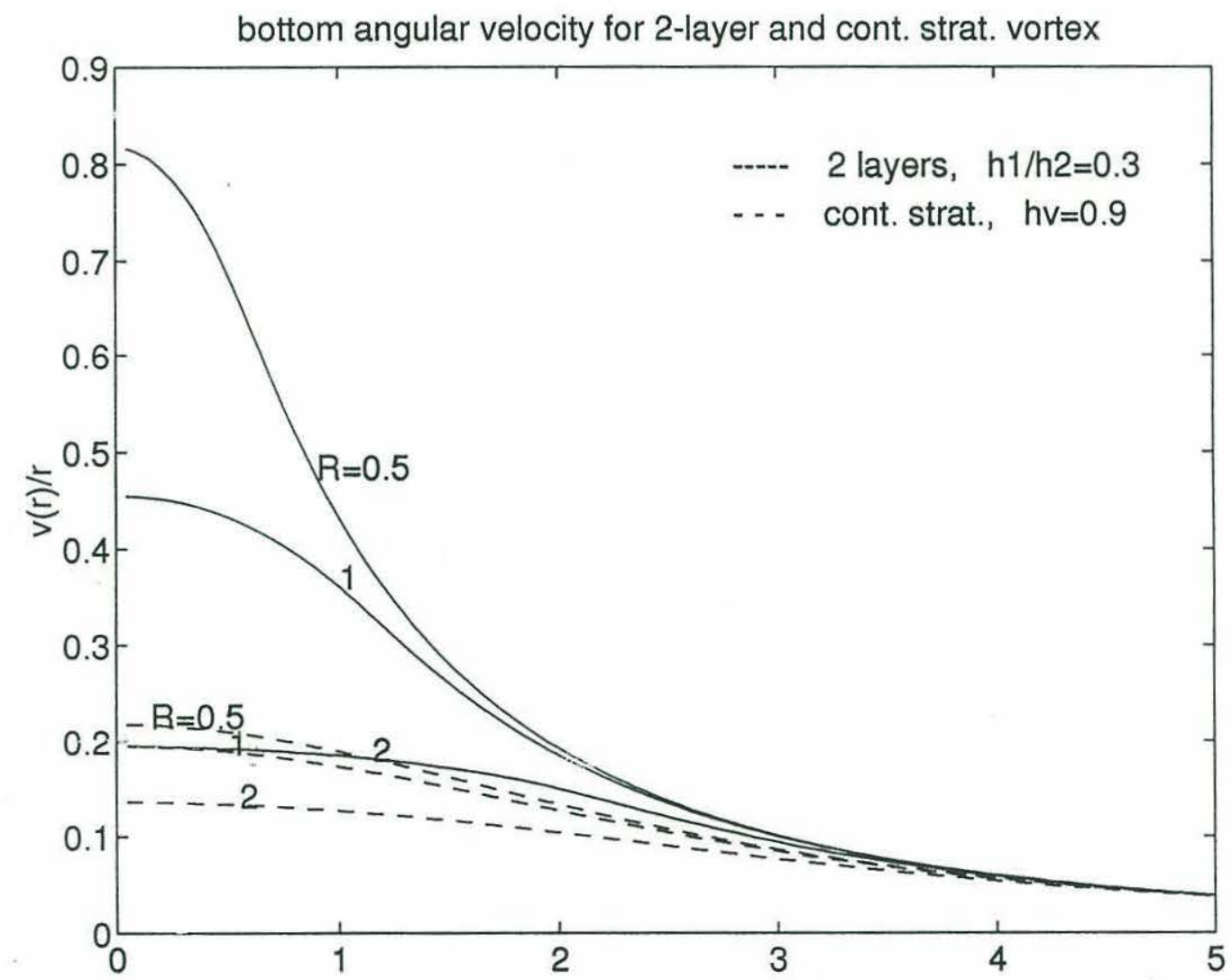

(b)

Figure 7.4(a,b). Velocities (a) and angullar velocities (b) at the bottom from the vortex for the two-layer and the continuously stratified model. 


\section{Chapter 8}

\section{Conclusions}

The present work is concerned with modeling of the interaction of an individual upper-ocean eddy with a smooth bottom topography simulating continental slopes. This is not the first study of such a process and some of my results conform with those in previous works, while others are considerably different from the conclusions based on other models. In particular my results suggest the primary importance of baroclinic effects and significant differences between the role of the planetary $\beta$-plane and a topographic slope in vortex motion. I also developed a new numerical technique for this and some other oceanographic problems. Here we want to reiterate the main points of this study, some novelties of the approach and the possible reasons for differences in the results of the current and previous studies.

1) Baroclinic effects. One of the important features of our approach is to investigate the baroclinic effects of the eddy-topography interaction process. In many previous works they were either absent or present in a more simple form. Baroclinic effects allow richer dynamical processes and the existence of qualitatively different regimes of a vortex-topography interaction. In particular our results show that isolated vortices over smooth, uniform topography and $\beta=0$ quickly reach steady state in which total barotropic vorticity of the flow is zero - we called this statement the "no net barotropic vorticity theorem". Thus baroclinic processes are not only important, but they are crucial in determining the vortex behavior, since barotropic flow is absent at all in this case. The "propulsion" mechanism of the vortex over topography is also essentially baroclinic, since a vortex forms a "hetonic" structure together with a vorticity field induced by itself because of the bottom slope.

2). Cross-slope motion. My calculations suggest that vortices can move mainly in the crossslope direction in the absence of other factors. Such motion is antisymmetric with respect to the sense of rotation of the vortices: cyclonic eddies move towards the shallower waters while anticyclones - to deeper parts of the basin. This coincides generally with other related studies, but what makes the presented results different is the along-slope component of vortex motion. In my model it can have either sign depending on different factors, like the curvature of the topographic slope or background flows. But this along-slope component is usually not dominant in comparison with the cross-slope one. This contrasts with the prevalent westward translation of eddies on the planetary beta-plane and the results of some other simulations of eddies over topography. 
3). The semi-lagrangian contour dynamics method. My main results were obtained with the numerical model constructed specifically to investigate this process of eddy-topography interaction. The model employs a technique resembling the method of a contour dynamics. Yet I want to accentuate here two of its features which distinguishes it from other models of this type. First instead of potential vorticity contours I use density contours at the bottom to represent the interaction of stratified fluid with topography more accurately. Second - I actually combine an Eulerian fixed grid and Lagrangian moving contours in an attempt to use the best features of them both. To do so at each timestep I interpolate density (or vorticity) field on the regular grid, perform the inversion procedure (computation of the velocity field) and interpolate the velocity field back to the moving contours. This technique allows to use some of the advantages of contour dynamics (following property-conserving parcels, reduction of partial to ordinary differential equations of contour evolution). At the same time one is rid of some of its limitations - for example a step-like vorticity distribution. Also the inclusion of the fixed grid allows us to build a much more efficient inversion procedure (using also a Green's function method) in comparison with the pure contour dynamics method.

Such a technique can be used not only for this problem but for many others as well. First of all it is very convenient for computation of any stratified flow with a uniform or simplified potential vorticity structure over a smooth topography. This can include, for example, strait or sill flows or the "Taylor column" problem. In these cases the problem remains computationally 2-dimensional. Then the same numerical procedure which I applied here to density contours at the bottom can be easily extended to density at the surface and PV contours in the interior and this allows us to compute more complex quasigeostrophic stratified flows. Moreover the described approach can be generalized to more complicated dynamics, in particular to the semi-geostrophic and primitive equations and the work is in progress in this direction to build a much more comprehensive model for oceanic flows.

4). Topographic slope vs. planetary $\beta$-plane. "Wave-gliding" vs. "wave-breaking" regimes. The role of a sloping bottom topography in oceanic circulation was often compared to that of the planetary $\beta$-effect. While this is certainly correct for a barotropic QG vorticity equation there is no exact equivalence of a sloping bottom and a planetary vorticity gradient when stratification is present. There are certainly some important similarities between these two cases: both support Rossby waves moving westward or to the left of the upslope direction with qualitatively similar dispersion relationships. Yet my results show that this qualitative similarity is not enough to ensure similar behavior of vortices over slope and on the $\beta$-plane. In my opinion it is the balance between the wave radiation and swirl velocity of the vortex that determines its motion. My suggestion is that for typical oceanic conditions eddies over the slope and on the planetary $\beta$-plane will fall into two dynamically different regimes with respect to this swirl-radiation balance. To distinguish these situations I introduced the concepts of the "wave-breaking" regime (for eddies on the $\beta$-plane) and the "wave- 
gliding" regime, characteristic for the baroclinic eddies over a slope. In the "wave-breaking" regime the eddy motion should be mainly in the westward direction for both cyclones and anticyclonic eddies, while in the "wave-gliding" regime the meridional, cross-slope velocity component is dominant and it is antisymmetric for vortices of different sign.

In the introductory chapter I mentioned the difficulties of comparison of the model results with observations. This stems mainly from the lack of deep-flow velocity or hydrographic data simultaneous with vortex motion and the influence of other important factors, unobserved or unaccounted for. Yet our results may help explain for example one intriguing fact about the circulation in the Eastern Mediterranian - the permanent presence of the "Shikmona" eddy in the Levantine basin. Our calculations show strong tendency of isolated anticyclonic eddies to move towards the deepest point in the basin - thus "stabilizing" its position in the center of a region with a bowl-shaped topography and preventing it from going away. This seaward tendency of anticyclones can also account for the fact that the Gulf Stream and Gulf of Mexico warm-core rings do not come close to the shore - typically they stay deeper of the 2000-m isobath, although their cores have considerably smaller vertical sizes.

In our study I used a variety of restricting assumptions - the quasigeostrophic f-plane approximation, a simple vortex structure, a uniform potential vorticity of ambient water. These results may not hold for more realistic circumstances, especially in the presence of strongly turbulent surroundings and irregularly-shaped seafloor. One can only speculate which of these results will survive and how can the behavior of vortices be changed in more complicated situations. Crossslope motion, particularly the seaward tendency of anticyclonic vortices appears to be a very robust feature of our results and I feel it will persist under more complicated conditions. Although eddies can never reach a steady state with vanishing barotropic flow, the compensating deep circulation should still develop and baroclinic effects will be important in any case. If the slope is too weak or the vortex is too close to the shore then the "wave-gliding" regime can be replaced by the "wavebreaking" one and the results of barotropic modeling can be more relevant (for example those of Wang, 1992). In this case the vortex behavior over a slope would more resemble its motion on the planetary beta-plane.

This study can be extended, using a more sophisticated model, in several directions. First, it would be interesting to examine the influence of rougher topography, more characteristic of the oceanic seafloor, on the vortex behavior. The sketchy results in the present study suggest that its motion can be strongly perturbed by the presence of topographic irregularities. Although the crossslope tendency remains, a trajectory can be more complicated and strongly depend on initial conditions and different parameters. This can explain the variety of vortex trajectories over continental slopes, particularly in the along-slope direction. Also the interaction of several vortices in the presence of a slope is worth studying. In my model isolated vortices rapidly developed a 
compensating circulation in the deeper flow and reached near-steady state. When other factors are present, like another vortex, the behavior can be more involved and a steady state can never be reached. But still the baroclinic effects considered in detail in the present study should play an important role even in the case of unsteady and turbulent environment. 


\section{Appendix 1}

\section{Parameterization of variable}

\section{buoyancy frequency}

\section{A1.1 Introduction}

It has long been recognized by meteorologists and oceanographers that the appropriate relation of a vertical to a horizontal scales for atmospheric and oceanic geostrophic turbulence should be their actual geometric relation multiplied by a ratio of a coriolis parameter to a buoyancy frequency $\mathrm{H}=\mathrm{Lf} / \mathrm{N}$. This corresponds to the so-called "Prandtl balance" (Prandtl, 1952) with a Burger number - a measure of relative importance of stretching term and relative vorticity - of the order one:

$$
\mathrm{B}=(\mathrm{NH} / \mathrm{fL})^{2} \approx 1
$$

Charney (1971) argued that when the vertical coordinate is rescaled accordingly, $\mathrm{z}=\mathrm{zN} / \mathrm{f}$, quasigeostrophic turbulence should be three-dimensionally isotropic and energy is equipartitioned between wavenumbers corresponding to all three coordinates. His arguments are based primarily on the fact that when the buoyancy frequency is uniform the operator relating a quasigeostrophic potential vorticity to a streamfunction is reduced to a simple three-dimensional laplacian in rescaled ("stretched") coordinates. Among other convenient properties of this constant $\mathrm{N}$ case is that the energy will be proportional to a squared three-dimensional gradient of a streamfunction.

This elegant transformation to a simple laplacian operator can be made rigorously only when a buoyancy frequency profile is uniform. When it is varying there is an additional term in the vertical part of the operator which does not generally allow an explicit analytical solution. This term is proportional to a vertical derivative of a buoyancy frequency and can be neglected when it changes only slightly at a characteristic vertical scale. For the real ocean it is apparently not true - in the main thermocline, for example, it can change dramatically over a few hundred meters. 
In this chapter we examine a possibility of generalization of the above mentioned approach to model the ocean with a non-uniform stratification profile. One can think of several motivations for doing this.

Usually for analytical and numerical studies the vertical mode expansion is used. This procedure is applicable to any stratification profiles but requires a numerical solution of SturmLouiville problem for vertical modes. Still it is often more convenient to have an analytical expression for vertical structure, dispersion relationship, etc., depending on several parameters of a profile, rather than to solve the vertical mode problem numerically for each individual case.

Moreover, growing popularity of lagrangian methods like contour dynamics suggest the need for the explicit formula for the Green's function and the azimuthal velocity profiles from a singular potential vorticity anomaly. One can, of course, expand it again into vertical modes (or layers) and retain only few of them for simplicity. This, however, can be rather inaccurate if we retain very few vertical modes, or complicated and inconvenient if we retain many of them. So it seems useful to have an explicit formula for continuously stratified case, similarly to a constant $\mathrm{N}$ model, without any truncation in the vertical.

\section{A1.2 "Parametric" buoyancy frequency profiles}

Consider a stratified rotating and horizontally unbounded ocean between a flat bottom at $\mathrm{z}=0$ and surface at $\mathrm{z}=\mathrm{H}$, with a constant coriolis parameter $\mathrm{f}$.

Generally the quasigeostrophic potential vorticity is related to the streamfunction via the elliptic operator

$$
\mathrm{q}=\left(\Delta_{\mathrm{h}}+\mathrm{L}_{\mathrm{z}}\right) \psi
$$

where $\Delta_{\mathrm{h}}=\partial_{\mathrm{xx}}^{2}+\partial_{\mathrm{yy}}^{2}$ - the horizontal Laplacian operator and the vertical operator $\mathrm{L}_{\mathrm{z}}$ is

$$
\mathrm{L}_{\mathrm{z}}=\partial_{\mathrm{z}} \frac{\mathrm{f}^{2}}{\mathrm{~N}^{2}(\mathrm{z})} \partial_{\mathrm{z}}
$$

Let's try to reduce (A1.1) to a more simple and convenient form:

$$
\tilde{\mathrm{q}}=\left(\Delta_{\mathrm{h}}+\partial_{\tilde{\mathrm{zz}}}^{2}-\lambda^{2}\right) \tilde{\psi}=\left(\Delta_{\mathrm{h}}+\widetilde{\mathrm{L}}_{\mathrm{z}}\right) \tilde{\psi}
$$

Here $\tilde{\mathrm{Z}}=\tilde{\mathrm{Z}}(\mathrm{z})$ is the transformed ("stretched") vertical coordinate, $\lambda$ is a real number and 


$$
\psi=\mu(\widetilde{\mathrm{z}}) \tilde{\psi}, \quad \mathrm{q}=\mu(\tilde{\mathrm{z}}) \tilde{\mathrm{q}}
$$

where $\mu(\widetilde{\mathbf{z}})$ is an "amplitude factor" dependent on the vertical coordinate only.

It is not quite obvious beforehand that such transformation from (A1.1) to (A1.1') can be accomplished for any profile $\mathrm{N}(\mathrm{z})$, so let's try to find a class of profiles for which it is possible. Changing vertical variable in the operator $\mathrm{L}_{\mathrm{z}}$ and substituting (A1.3) we shall obtain:

$$
\frac{\partial}{\partial \mathrm{z}} \frac{\mathrm{f}^{2}}{\mathrm{~N}^{2}} \frac{\partial}{\partial \mathrm{z}} \psi=\mathrm{f}^{2} \eta \frac{\partial}{\partial \tilde{\mathrm{z}}} \eta \frac{1}{\mathrm{~N}^{2}} \frac{\partial}{\partial \tilde{\mathrm{z}}} \mu \tilde{\psi}, \quad\left(\eta \equiv \frac{\partial \tilde{\mathrm{z}}}{\partial \mathrm{z}}\right)
$$

From now on we shall denote $\frac{\partial}{\partial \tilde{z}}(*)$ by prime $(*)^{\prime}$ for simplicity and our vertical operator becomes:

$$
\begin{gathered}
\widetilde{\mathrm{L}}_{\tilde{\mathrm{z}}}=\mathrm{f}^{2} \eta \frac{\partial}{\partial \tilde{\mathrm{z}}} \eta \frac{1}{\mathrm{~N}^{2}} \frac{\partial}{\partial \tilde{\mathrm{z}}} \mu \tilde{\psi}=\mathrm{f}^{2} \eta\left(\frac{\eta}{\mathrm{N}^{2}}(\mu \tilde{\psi})^{\prime}\right)^{\prime}= \\
=\mathrm{f}^{2} \eta\left(\left(\frac{\eta^{\prime}}{\mathrm{N}^{2}}-2 \eta \frac{\mathrm{N}^{\prime}}{\mathrm{N}^{3}}\right)\left(\mu^{\prime} \tilde{\psi}+\mu \tilde{\psi}^{\prime}\right)+\frac{\eta}{\mathrm{N}^{2}}\left(\mu^{\prime \prime} \tilde{\psi}+2 \mu^{\prime} \tilde{\psi}^{\prime}+\mu \tilde{\psi}^{\prime \prime}\right)\right)= \\
=\frac{\mathrm{f}^{2}}{\mathrm{~N}^{2}} \eta\left(\eta \mu \tilde{\psi}^{\prime \prime}+\left[\left(\eta^{\prime}-2 \eta \frac{\mathrm{N}^{\prime}}{\mathrm{N}}\right) \mu+2 \eta \mu^{\prime}\right] \tilde{\psi}^{\prime}+\left[\left(\eta^{\prime}-2 \eta \frac{\mathrm{N}^{\prime}}{\mathrm{N}}\right) \mu^{\prime}+\eta \mu^{\prime \prime}\right] \tilde{\psi}\right)
\end{gathered}
$$

To get a "pure Laplacian" or, more generally, "pure Helmholtzian" operator of (A1.1') this long expression must be reduced to a simple second vertical derivative minus $\lambda^{2}$ multiplied by the rescaled (tilded) streamfunction: $\tilde{\psi}^{\prime \prime}-\lambda^{2} \tilde{\psi}$. To achieve this we must satisfy the following set of equations:

$$
\begin{aligned}
& \frac{\mathrm{f}^{2}}{\mathrm{~N}^{2}} \eta^{2}=1 \\
& \frac{\mathrm{f}^{2}}{\mathrm{~N}^{2}} \eta\left(\left(\eta^{\prime}-2 \eta \frac{\mathrm{N}^{\prime}}{\mathrm{N}}\right) \mu+2 \eta \mu^{\prime}\right)=0 \\
& \frac{\mathrm{f}^{2}}{\mathrm{~N}^{2}} \eta\left(\left(\eta^{\prime}-2 \eta \frac{\mathrm{N}^{\prime}}{\mathrm{N}}\right) \mu^{\prime}+\eta \mu^{\prime \prime}\right)=-\lambda^{2} \mu
\end{aligned}
$$

From (A1.4a) we have $\eta=\frac{\partial \tilde{z}}{\partial z}= \pm \frac{N}{f}, \quad$ natural choice is " + ", so that $\eta=\frac{N}{f}$. 
This means that the coordinate $\tilde{\mathrm{z}}$ must be "differentially" rescaled $d \widetilde{\mathrm{z}}=\frac{\mathrm{N}(\mathrm{z})}{\mathrm{f}} d \mathrm{z}$. This rescaling was suggested, for example, by Flierl (1987) although he did not specify how and for which profiles this can be accomplished.

Such scaling implies a rather peculiar property of this rescaled coordinate $\tilde{\mathrm{z}}$ which appears to be the "natural" vertical coordinate for quasigeostrophic motion. An increment of this coordinate $\tilde{\mathrm{z}}$ is proportional to the buoyancy frequency $\mathrm{N}$ and an increment of geometric coordinate $\mathrm{z}$, so it is proportional to a square root of an increment of density. It means that this "natural" coordinate $\tilde{\mathbf{z}}$ is neither density nor the geometric coordinate $\mathrm{z}$ but in some sense a geometric mean of those two. This fact can suggest, for example, the proper vertical grid spacing for level or layered numerical models.

From the two other equations of (A1.4) we have:

$$
\begin{gathered}
\frac{N^{\prime}}{N}=2 \frac{\mu^{\prime}}{\mu} \\
\mu^{\prime \prime}-\frac{N^{\prime}}{N} \mu^{\prime}+\lambda^{2} \mu=0
\end{gathered}
$$

Introducing new variables $\mathrm{n}=\frac{\mathrm{N}^{\prime}}{\mathrm{N}}$ and $\mathrm{m}=\frac{\mu^{\prime}}{\mu}$ we obtain a simple system of equations:

$$
\begin{gathered}
n=2 m \\
m^{\prime}-m^{2}+\lambda^{2}=0
\end{gathered}
$$

Now consider two cases separately, with a zero and non-zero parameter $\lambda$ :

\section{1) $\underline{\lambda=0}$.}

Integrating $\left(\mathrm{A} 1.5 \mathrm{~b}^{\prime}\right)$ one time we get $\quad \frac{\mathrm{m}}{\mathrm{m}_{0}}=\frac{1}{\left(1-\mathrm{m}_{0} \tilde{\mathrm{z}}\right)}$, where $\quad \mathrm{m}_{0}=\left.\frac{\mu^{\prime}}{\mu}\right|_{\tilde{z}=0}$ parameter of a profile, $\mathrm{N}_{0}=\mathrm{N}(0), \mu_{0}=\mu(0)$. Integrating this relation ones more (recall that $\mathrm{m}=\frac{\mu^{\prime}}{\mu}$ ) and taking into account (A1.5a') we easily obtain:

$$
\frac{\mathrm{N}}{\mathrm{N}_{0}}=\frac{1}{\left(1-\mathrm{m}_{0} \tilde{\mathrm{z}}\right)^{2}}, \quad \frac{\mu}{\mu_{0}}=\frac{1}{1-\mathrm{m}_{0} \tilde{\mathrm{z}}}
$$


Switching to the physical coordinate $\mathrm{z}$ we get

$$
\frac{\mathrm{N}}{\mathrm{N}_{0}}=\left(1-3 \mathrm{~m}_{0} \frac{\mathrm{N}_{0}}{\mathrm{f}} \mathrm{z}\right)^{-2 / 3}, \quad \frac{\mu}{\mu_{0}}=\left(1-3 \mathrm{~m}_{0} \frac{\mathrm{N}_{0}}{\mathrm{f}} \mathrm{z}\right)^{-1 / 3}
$$

and two vertical coordinates are connected by the relation

$$
\tilde{\mathrm{z}}=\frac{1}{\mathrm{~m}_{0}}\left(1-\left(1-3 \mathrm{~m}_{0} \frac{\mathrm{N}_{0}}{\mathrm{f}} \mathrm{z}\right)^{-1 / 3}\right)
$$

Examples of profiles with different $\mathrm{m}_{0}$ and $\lambda=0$ are given in figure A1.1a. The profile parameter $m_{0}$ can easily be substituted by the ratio $N_{\text {surface }} / N_{\text {bottom }}$ : from (A1.6) we easily obtain

$$
\frac{\mathrm{N}_{\text {surface }}}{\mathrm{N}_{\text {bottom }}}=\frac{1}{1-\mathrm{m}_{0}}
$$

Here and later we assume that the stretched variable $\tilde{\mathrm{z}}$ is normalized by the "stretched" depth $\widetilde{\mathrm{H}}$ of an ocean, which will be our unit lengthscale:

$$
\widetilde{\mathrm{H}}=\int_{0}^{\mathrm{H}} \frac{\mathrm{N}(\mathrm{z})}{\mathrm{f}} d \mathrm{z}
$$

So that at the bottom $\tilde{\mathrm{z}}=0$ and at the surface $\tilde{\mathrm{z}}=1$. All distances and wavenumbers are assumed to be nondimensionalized accordingly.

\section{2) $\underline{\lambda \neq 0}$.}

Integration is a little bit more difficult this time but finally we can obtain:

$$
\frac{N}{N_{0}}=\frac{\lambda^{2}}{\left(\lambda \cosh (\lambda \tilde{z})-m_{0} \sinh (\lambda \tilde{z})\right)^{2}}, \quad \frac{\mu}{\mu_{0}}=\frac{\lambda}{\lambda \cosh (\lambda \tilde{z})-m_{0} \sinh (\lambda \tilde{z})}
$$

and the two vertical coordinates are connected by the relation: 


$$
\frac{1}{\lambda} \sinh (\lambda \tilde{z})\left(\left(m_{0}^{2}+\lambda^{2}\right) \sinh (\lambda \widetilde{z})-2 m_{0} \lambda \cosh (\lambda \tilde{z})\right)-2\left(m_{0}^{2}-\lambda^{2}\right) \tilde{z}=2 m_{0} \lambda z
$$

It can easily be shown that when $\lambda$ tends to zero the relations (A1.8) and (A1.9) are reduced to (A1.6) and (A1.7) correspondingly.

So we have a family of profiles $\mathrm{N}(\mathrm{z})$ determined by the relation (A1.8) and dependent on two parameters $-\mathrm{m}_{0}$ and $\lambda$. The parameter $\mathrm{m}_{0}$ is proportional to a relative vertical derivative of $\mathrm{N}(\tilde{\mathrm{z}})$ at the bottom (in "stretched" coordinates) - it is half tangent of the angle at which profile $\mathrm{N}(\tilde{\mathrm{z}}) / \mathrm{N}_{0}$ intersects the bottom (this follows from (A1.5a)):

$$
\mathrm{m}_{0}=\mu_{\tilde{z}}(0)=\frac{1}{2} \frac{\mathrm{N}_{\mathbf{z}}(0)}{\mathrm{N}(0)}
$$

The other parameter - $\lambda$ has the meaning of (imaginary) wavenumber in the Helmholtz operator (A1.1'). Since both parameters $\mathrm{m}_{0}$ and $\lambda$ have the meaning of inverse lengthscales it is convenient to retain one parameter of this dimension and substitute another one by their ratio $\tau$ so that

$$
\tau=\mathrm{m}_{0} / \lambda
$$

Then we can rewrite the expressions (A1.8) and (A1.9) in the form:

$$
\frac{\mathrm{N}}{\mathrm{N}_{0}}=\frac{1}{\left(\cosh \left(\tau \mathrm{m}_{0} \tilde{\mathrm{z}}\right)-\frac{1}{\tau} \sinh \left(\tau \mathrm{m}_{0} \tilde{\mathrm{z}}\right)\right)^{2}}, \quad \frac{\mu}{\mu_{0}}=\frac{1}{\cosh \left(\tau \mathrm{m}_{0} \tilde{\mathrm{z}}\right)-\frac{1}{\tau} \sinh \left(\tau \mathrm{m}_{0} \tilde{\mathrm{z}}\right)}
$$



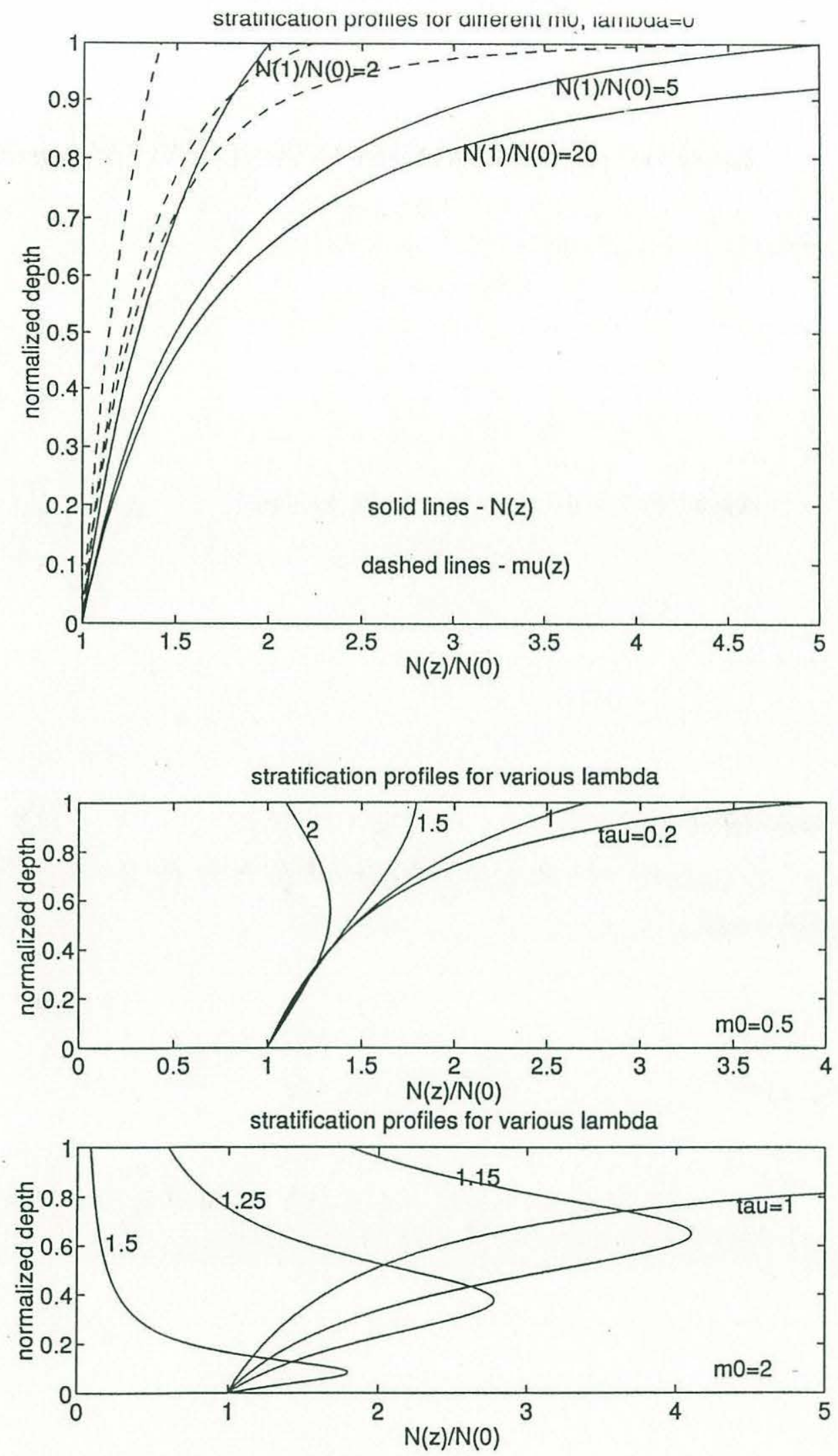

Figure A1.1 Examples of the "parametric" stratification profiles
(a) for $\lambda=0$
(b) for different $\lambda$ 


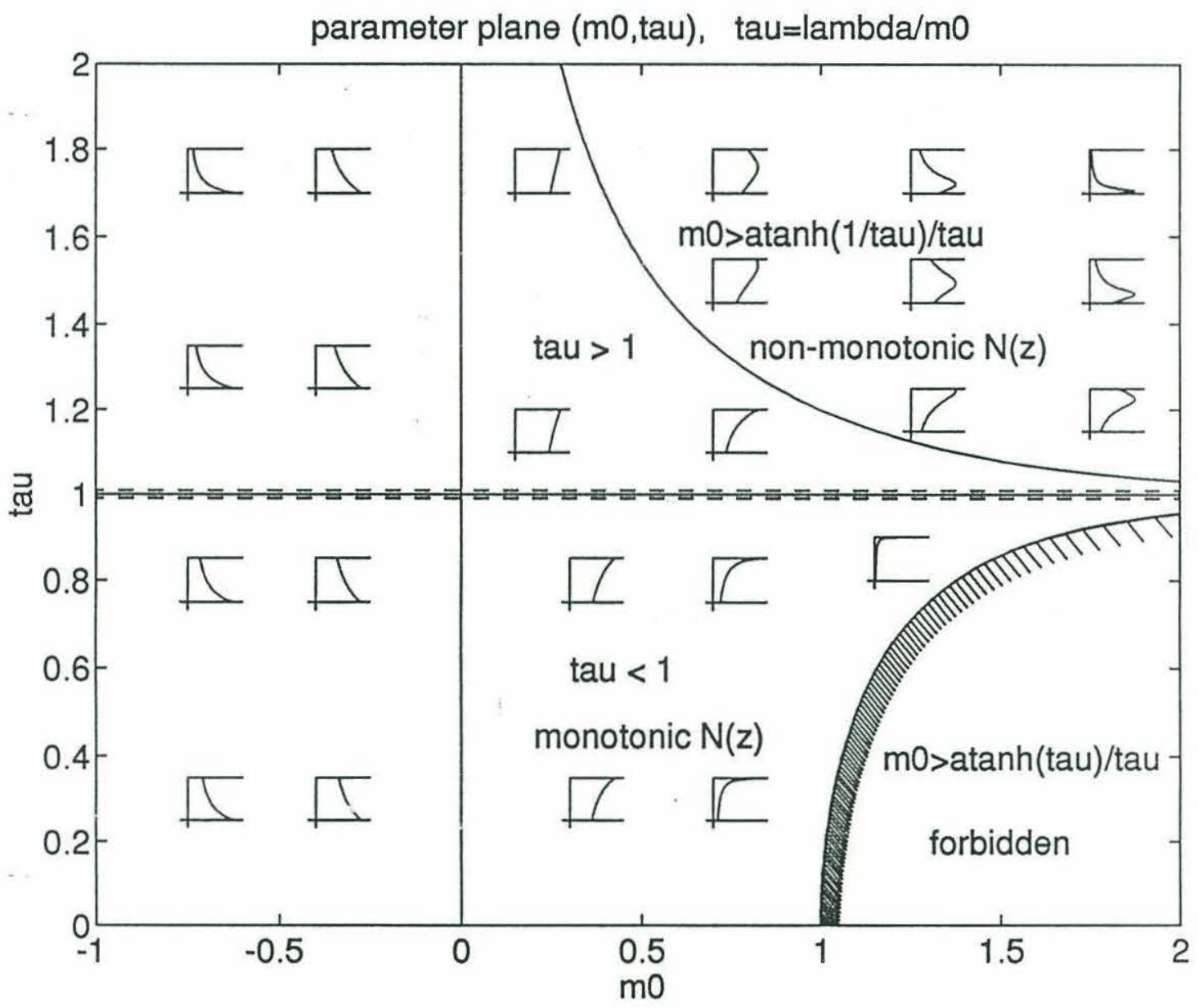

Figure A1.2 The parameter plane $\left(\mathrm{m}_{0}, \tau\right)$ 


$$
\mathrm{z}=\frac{1}{2 \tau^{2}} \sinh \left(\tau \mathrm{m}_{0} \tilde{\mathrm{z}}\right)\left(\left(1+\tau^{2}\right) \sinh \left(\tau \mathrm{m}_{0} \tilde{\mathrm{z}}\right)-2 \tau \cosh \left(\tau \mathrm{m}_{0} \tilde{\mathrm{z}}\right)\right)-\frac{\left(1-\tau^{2}\right)}{\tau} \tilde{\mathrm{z}}
$$

Examples of stratification profiles and the "amplitude factor" dependence on the vertical coordinate $\mathrm{z}$ are shown in figure A1.1. Figure A1.1a shows profiles with $\lambda=0$ and different values of the parameter $m_{0}$. In this case all profiles are monotonic and if $m_{0}>0 \mathrm{~N}(\mathrm{z})$ increases with z. This can represent gross structure of typical oceanic stratification profile, with upper ocean buoyancy frequency much greater than its value near the bottom. Corresponding profiles of "amplitude factor" $\mu(\mathrm{z})$ are shown in figure A1.1a by dashed lines. Its $\mathrm{z}$ dependence is proportional to square root of $\mathrm{N}(\mathrm{z})$ as seen from relations (A1.6) and (A1.8) so that it varies slower than the buoyancy frequency itself.

Figure A1.1b shows examples of the profiles with a non-zero parameter $\lambda$ (or, equivalently, $\tau)$. In this case the situation is more complicated. When $\tau<1$ profiles are qualitatively similar to previous parametric case of $\tau=0$ with monotonic behavior of $\mathrm{N}(\mathrm{z})$. For $\mathrm{m}_{0}>0$ buoyancy frequency monotonically increases toward the surface, when $m_{0}<0$ it decreases. But when $\tau>1$ profiles can have a non-monotonic curvature and for $m_{0} \geq \operatorname{atanh}(1 / \tau) / \tau$ profiles themselves can be non-monotonic, with maximum $\mathrm{N}(\mathrm{z})$ in the interior. By choosing suitable parameters $\tau$ and $\mathrm{m}_{0}$ one can obtain a profile more closely resembling the actual stratification - with a sharp maximum of $\mathrm{N}(\mathrm{z})$ near the surface, representing the thermocline structure.

Not all parameters $m_{0}$ and $\tau$ are permissible to construct a stratification profile. We should choose among those which render denominators in (A1.8') positive. Qualitative behavior of profiles is sketched in figure A1.2 in the $\left(\mathrm{m}_{0}, \tau\right)$ parameter plane. Profiles are invariant under a transformation $\tau \rightarrow-\tau$ so only positive values of $\tau$ is shown. Area $m_{0} \geq \operatorname{atanh}(\tau) / \tau$ is forbidden because the denominator is not positive. To the left of this line and also to the left of the line $\mathrm{m}_{0}=\operatorname{atanh}(1 / \tau) / \tau$ they are monotonic, below $\tau=1$ and also for $\mathrm{m}_{0}<0$ and any $\tau$ the curvature of the profiles does not change sign. When $m_{0}>\operatorname{atanh}(1 / \tau) / \tau$ profiles can have an internal maximum (but not a minimum) and this part of the parameter plane is most interesting to construct a profile resembling a realistic behavior $N(z)$. The line $\tau=1$ is also not permissible. All negative values of $m_{0}$ are permissible and profiles are monotonically decreasing in this case.

Summarizing this section we shall reformulate the boundary-value problem relating the QG potential vorticity with the streamfunction for parametric buoyancy profiles in the "stretched" coordinates. Because of the introduction of the "amplitude factor" $\mu(\mathrm{z})$ the vertical derivative of the streamfunction needs to be modified:

$$
\psi_{\mathrm{z}}=(\mu \tilde{\psi})_{\mathrm{z}}=\frac{\mathrm{N}}{\mathrm{f}}(\mu \tilde{\psi})_{\tilde{\mathrm{z}}}=\frac{\mathrm{N}}{\mathrm{f}}\left(\mu_{\tilde{\mathrm{z}}} \tilde{\psi}+\mu \tilde{\psi_{\mathrm{z}}}\right)=\mu \frac{\mathrm{N}}{\mathrm{f}}\left(\mathrm{m} \tilde{\psi}+\tilde{\psi_{\mathrm{z}}}\right)
$$


therefore the "rigid lid" boundary condition at the surface and the bottom can be written as

$$
\begin{aligned}
& \tilde{\psi}+\mathrm{S}_{0} \tilde{\psi} \tilde{\mathrm{z}}=0 \quad @ \quad \tilde{\mathrm{z}}=0 \\
& \tilde{\psi}+\mathrm{S}_{1} \tilde{\psi}_{\tilde{\mathrm{z}}}=0 \quad @ \quad \tilde{\mathrm{z}}=1
\end{aligned}
$$

where we introduced constants $\mathrm{S}_{0}=\frac{1}{\mathrm{~m}_{0}} \quad$ and $\quad \mathrm{S}_{1}=\frac{1}{\mathrm{~m}_{1}}$ for convenience. These relations together with the Helmholtz operator (A1.1') comprise the above mentioned boundary-value problem.

When $\tau=0$ we have $\mathrm{m}_{1}=\frac{\mathrm{m}_{0}}{1-\mathrm{m}_{0}}$ and $\mathrm{S}_{0}-\mathrm{S}_{1}=1 \quad$ - a useful relation which we shall use in the next section where we shall consider vertical modes and Rossby waves structure for the "parametric" profiles obtained above.

\section{A1.3 Vertical structure and dispersion relationship for topographic and planetary Rossby waves}

Using the parametric approach discussed in the previous section we can derive a general analytical formulae for quasigeostrophic vertical modes and dispersion relations for planetary and topographic Rossby waves for the stratification profiles which are given by the equations (A1.6) or (A1.8).

1) Planetary Rossby waves.

For planetary Rossby waves the vertical mode structure is given by a solution of a Stürm Louiville problem:

$$
\begin{aligned}
& \mathrm{L}_{\mathrm{z}} \psi(\mathrm{z})=-\gamma^{2} \psi(\mathrm{z}) \\
& \psi_{\mathrm{z}}=0 \quad \text { @ } \mathrm{z}=0 \text { and } \mathrm{z}=\mathrm{H}
\end{aligned}
$$

where $-\gamma^{2}$ is an eigenvalue of the vertical operator $\mathrm{L}_{\mathrm{z}}(\gamma), \gamma$ corresponds to an inverse Rossby deformation radius), $\mathrm{H}$ is a depth of an ocean. Changing to the tilded variables $\psi \rightarrow \tilde{\psi}, \mathrm{z} \rightarrow \tilde{\mathrm{z}}$, $\mathrm{L}_{\mathrm{z}} \rightarrow \mathrm{L}_{\tilde{\mathrm{z}}}=\partial_{\tilde{\mathrm{z}}}^{2}-\lambda^{2}$, we have now the following problem: 


$$
\begin{array}{lrl}
\tilde{\Psi} \tilde{\mathrm{zz}}+\kappa^{2} \tilde{\psi}=0, & \text { where } & \kappa^{2}=\gamma^{2}-\lambda^{2} \\
\widetilde{\psi}+\mathrm{S}_{0} \tilde{\Psi}_{\tilde{\mathrm{z}}}=0 & \text { @ } & \tilde{\mathrm{z}}=0 \\
\tilde{\Psi}+\mathrm{S}_{1} \tilde{\Psi}_{\tilde{\mathrm{z}}}=0 & \text { @ } & \tilde{\mathrm{z}}=1
\end{array}
$$

An obvious solution is $\tilde{\psi}=\mathrm{A} \cos \kappa \tilde{\mathrm{z}}+\mathrm{B} \sin \kappa \tilde{\mathrm{z}}$ so that $\tilde{\psi}_{\tilde{\mathrm{z}}}=\kappa(-\mathrm{A} \sin \kappa \tilde{z}+\mathrm{B} \cos \kappa \tilde{\mathrm{z}})$, and from the boundary conditions we obtain:

$$
\mathrm{A}+\mathrm{B} \mathrm{S}_{0} \kappa=0
$$

$A\left(\cos \kappa-S_{0} \kappa \sin \kappa\right)+B\left(\sin \kappa+S_{1} \kappa \cos \kappa\right)=0$

The compatibility condition (a zero determinant) gives us the dispersion relationship:

$$
\tan \kappa=\frac{\kappa\left(S_{0}-S_{1}\right)}{1+S_{0}^{2} \kappa^{2}}
$$

and vertical eigenmodes have the form:

$$
\tilde{\psi}=\mathrm{A}\left(\cos \kappa \tilde{z}-\frac{1}{\mathrm{~S}_{0} \kappa} \sin \kappa \tilde{z}\right)
$$

for each $\kappa$ satisfying the dispersion relation (A1.12).

If $\lambda=0$ then $\kappa=\gamma$ and $S_{0}=\frac{1}{m_{0}}, S_{1}=\frac{1}{m_{0}}-1, S_{0}-S_{1}=1$ and the last formula has the form: 
eigenvalues for vertical modes, lambda $=0$
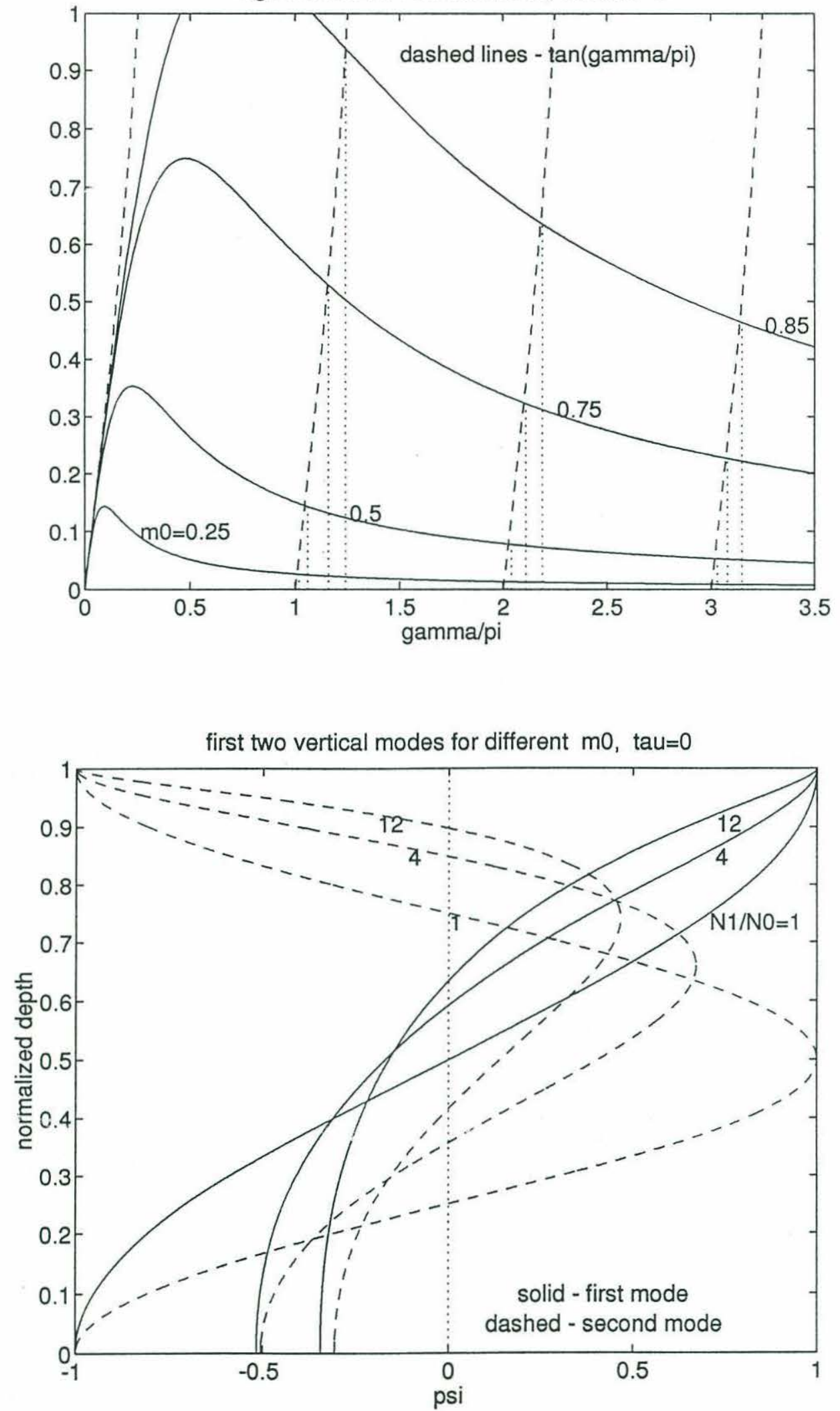

Figure A1.3 Quasigeostrophic vertical modes for "parametric" profiles:

(a) graphical solution of for deformation radius;

(b) examples of vertical modes for $\lambda=0$ 


$$
\begin{gathered}
\tan \gamma=\frac{\gamma}{1+\gamma^{2} / m_{0}^{2}} \\
\tilde{\psi}=A\left(\cos \gamma \tilde{z}-\frac{m_{0}}{\gamma} \sin \gamma \tilde{z}\right)
\end{gathered}
$$

Figure A1.3a shows graphical solutions for the dispersion relation (A1.12'). Left-hand side of the equation (A1.12') as a function of $\gamma$ (tangent lines) is shown by dashed lines. Several curves corresponding to the right-hand side at different $\mathrm{m}_{0}$ are shown by solid lines. Projections of the intersections of these curves on the $\gamma$ axis (scaled by $\pi$ ) determine the solutions to the dispersion relation (A1.12'). For the case of uniform stratification solutions correspond to the lower end of tangent lines - that is $\gamma_{n}=\pi n$ where $n$ is integer. One can see that values of $\gamma$ are slightly greater than those for a uniform profile, which means that corresponding deformation radii are slightly smaller for non-uniform stratification than for constant $\mathrm{N}$ cases of an equal rescaled depth $\widetilde{\mathrm{H}}$. Examples of vertical modes are shown in figure A1.3b for several positive values of $\mathrm{m}_{0}$ (which means that the buoyancy frequency increases toward the surface). They naturally have a more pronounced structure in the upper part and change slowly near the bottom. Their behavior near the surface $(\tilde{\mathrm{z}}=1)$ seems quite peculiar since they do not seem to intersect the line $\tilde{\mathrm{z}}=1$ perpendicularly (rigid-lid condition). More careful analysis indicates however that they do satisfy this condition, but turn to the right angle very close to the surface in the case of a large ratio of the surface to bottom buoyancy frequency. This happens because $N(z)$ changes very rapidly near the surface, so do the profiles of vertical modes.

\section{2) Topographic Rossby waves.}

For the case of topographic Rossby waves the procedure is similar. But now we do not need to solve an eigenvalue problem for deformation radius; instead we must solve a dispersion relation for a phase velocity $\mathrm{c}$ which appears as a coefficient in the bottom boundary condition. Now the vertical structure satisfies the equation:

$$
\left(-\kappa^{2}+L_{z}\right) \psi(z)=0
$$

and the boundary conditions

$$
\psi+\widehat{S} \psi_{\mathrm{z}}=0 \quad @ \mathrm{z}=0
$$




$$
\psi_{\mathrm{z}}=0 \quad \text { @ } \mathrm{z}=\mathrm{H}
$$

Here the parameter $\widehat{\mathrm{S}}$ is proportional to the phase velocity $\quad \mathrm{c}: \widehat{\mathrm{S}}=\mathrm{cf} / \alpha \mathrm{N}_{0}^{2}$, where $\quad \alpha$ is slope angle and $\mathrm{N}_{0}$ - the buoyancy frequency at the bottom. Switching to the stretched coordinate $\tilde{\mathrm{z}}$ and tilded variables and recalling (A1.10) the bottom boundary condition (A1.14b) can be written as

$$
\left(1+\widehat{\mathrm{S}} \frac{\mathrm{N}_{0}}{\mathrm{f}} \mathrm{m}_{0}\right) \tilde{\psi}+\widehat{\mathrm{S}} \frac{\mathrm{N}_{0}}{\mathrm{f}} \tilde{\Psi} \tilde{z}=0
$$

After some simple transformations this system will be written as follows:

$$
\begin{array}{lcc}
\tilde{\psi}_{\tilde{\mathrm{zz}}}-v^{2} \tilde{\psi}=0, & \text { where } & v^{2}=\kappa^{2}+\lambda^{2} \\
\tilde{\psi}+\mathrm{S}_{0} \Gamma \tilde{\psi} \tilde{\mathrm{z}}=0 & \text { @ } & \tilde{\mathrm{z}}=0 \\
\tilde{\psi}+\mathrm{S}_{1} \tilde{\psi}_{\tilde{\mathrm{z}}}=0 & \text { @ } & \tilde{\mathrm{z}}=1
\end{array}
$$

where the constant $\Gamma=\frac{\widetilde{\mathrm{S}}}{\mathrm{S}_{0}+\widetilde{\mathrm{S}}}, \quad \widetilde{\mathrm{S}}=\frac{\omega}{\alpha \mathrm{N}_{0} \kappa} \quad$ is a nondimensional wave frequency.

In this case the solution will have the form $\tilde{\psi}=\mathrm{A} \cosh \kappa \tilde{z}+\mathrm{B} \sinh \kappa \tilde{z}$. Similarly to the case of planetary waves we obtain the dispersion relation and the vertical structure:

$$
\tanh v=\frac{v\left(\Gamma S_{0}-S_{1}\right)}{1+\Gamma S_{0}^{2} v^{2}}
$$

which we must solve for $\Gamma$ and then $\widetilde{\mathrm{S}}$ :

$$
\widetilde{\mathrm{S}}=\frac{1+\mathrm{m}_{1} \tanh (v) / v}{v \tanh (v)+\mathrm{m}_{1}-\mathrm{m}_{0}-\mathrm{m}_{0} \mathrm{~m}_{1} \tanh (v) / v}
$$

The wave frequency (dimensional) can be expressed through $\widetilde{\mathrm{S}}$ :

$$
\omega=\alpha \mathrm{N}_{0} \widetilde{\mathrm{S}} \text {. }
$$



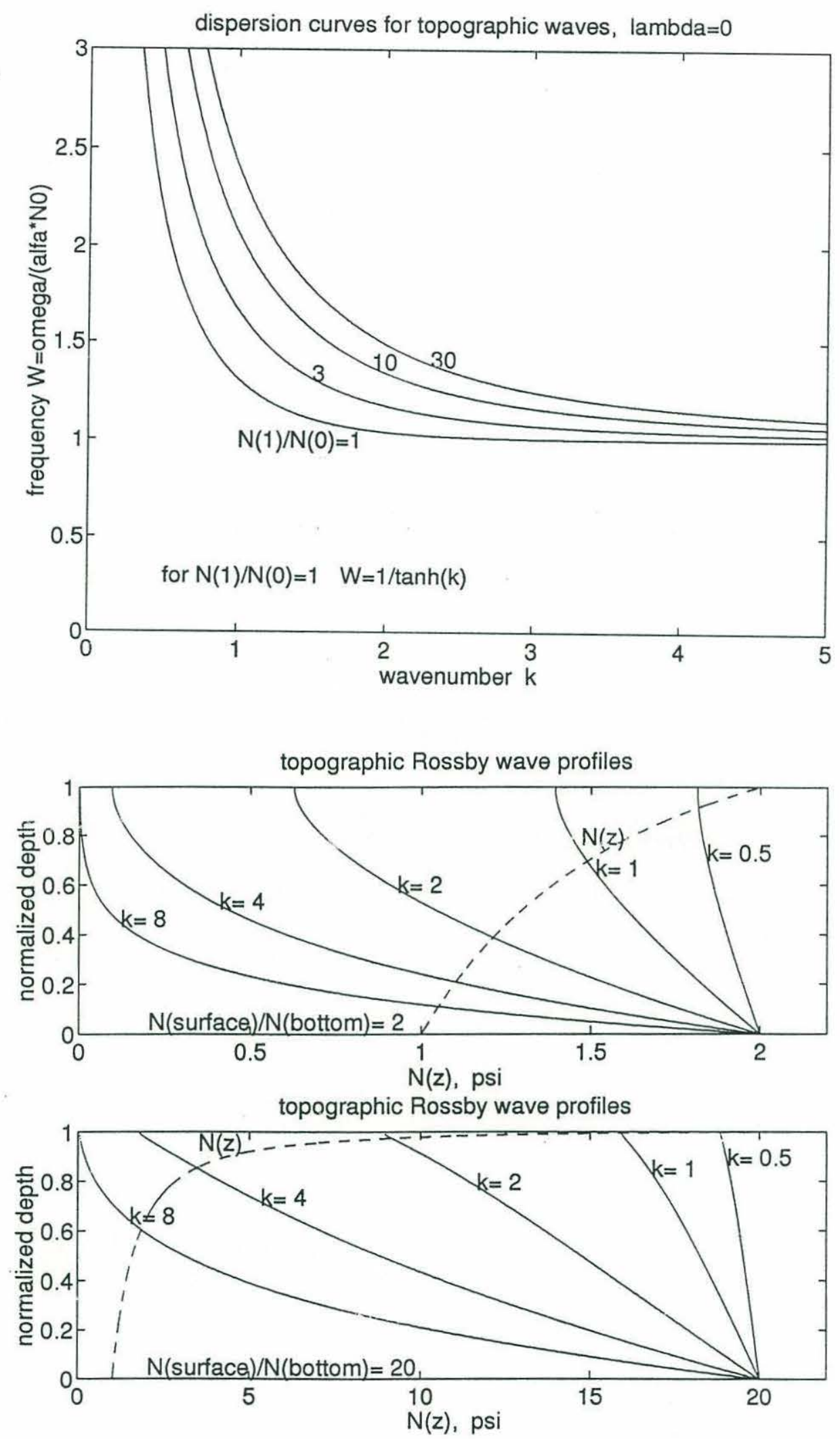

Figure A1.4 Topographic Rossby waves for "parametric" stratification:

(a) dispersion relation;

(b) vertical structure. 
The vertical structure will be given by the following expression:

$$
\tilde{\psi}=\mathrm{A}\left(\cosh v \tilde{\mathrm{Z}}-\frac{1}{\Gamma S_{0} v} \sinh v \tilde{\mathrm{Z}}\right)
$$

When $\lambda=0$ we have $v=\kappa$ and relations (A1.15) - (A1.17) are reduced to:

$$
\begin{gathered}
\tanh \kappa=\frac{\mathrm{m}_{0}\left(1+\mathrm{m}_{0}-\Gamma\right)}{\mathrm{m}_{0}^{2}+\Gamma\left(1-\mathrm{m}_{0}\right) \kappa^{2}} \\
\widetilde{\mathrm{S}}=\frac{1-\mathrm{m}_{0}(1-\tanh (\kappa) / \kappa)}{\left(1-\mathrm{m}_{0}\right) \kappa \tanh (\kappa)+\mathrm{m}_{0}(1-\tanh (\kappa) / \kappa)} \\
\tilde{\psi}=\mathrm{A}\left(\cosh \kappa \tilde{\mathrm{z}}-\Gamma \frac{\mathrm{m}_{0}}{\kappa} \sinh \kappa \tilde{\mathrm{z}}\right)
\end{gathered}
$$

In figure A1.4a we show dispersion curves $\widetilde{\mathrm{S}}$ as functions of the alongslope wavenumber $\kappa$ for several values of stratification parameters $\mathrm{m}_{0}$ and $\lambda$. One can see that for typical stratification profiles increasing towards the surface, wave frequency is slightly larger than for uniform stratification profiles for corresponding wavenumbers. Figure A1.4b shows some examples of vertical structure of topographic Rossby waves. One can see that for positive $m_{0}$ it inclines steeper near the surface and typical profile is almost linear with depth rather than having greater curvature near the bottom as in the case of uniform stratification. This is understandable from a point of view that profiles should be more barotropic near the bottom since stratification is weaker there and should have more structure near the surface - where stratification is strong and varies rapidly.

\section{A1.4 Green's function and azimuthal velocity for the case of parametric variable stratification}

To solve the boundary value problem (A1.1') - (A1.11a,b) we must invert potential vorticity to obtain the streamfunction and the velocity field. In the case of the uniform stratification one can do it easily by a convolution of a potential vorticity with the Green's function which in this case is equivalent to a field induced by a point charge plus an infinite system of images symmetric relative to a flat bottom and a surface of an ocean. 
In this section we attempt to derive the Green's function and, correspondingly, the azimuthal velocity profile when stratification is not uniform but belongs to the family of parametric profiles described in the previous sections. We restrict our analysis however to the case $\lambda=0$ for simplicity because the problem appears already tedious enough. Of course the approach described below can be straightforwardly generalized to the case with non-zero parameter $\lambda$.

Generally Green's function depends on coordinates of both "source" ( $\left.x^{\prime}, y^{\prime}, z^{\prime}\right)$ and "observing point" $(\mathrm{x}, \mathrm{y}, \mathrm{z})$, so that $\mathrm{G}=\mathrm{G}\left(\mathrm{x}, \mathrm{y}, \mathrm{z}, \mathrm{x}^{\prime}, \mathrm{y}^{\prime}, \mathrm{z}^{\prime}\right)$. If the domain is translationally and rotationally invariant in horizontal coordinates, like in our case, then $G=G\left(\left|r-r^{\prime}\right|, z, z^{\prime}\right)$, where $\left|r-r^{\prime}\right|=\left(x-x^{\prime}\right)^{2}+\left(y-y^{\prime}\right)^{2}$. To simplify notation we assume that "source" is placed at a point $(0,0, \mathrm{~h})$, so that we shall seek our Green's function in the form $G=G(r, z, h)$ where $r^{2}=x^{2}+y^{2}$.

This Green's function must render the interior laplacian operator singular at a given point $(0,0, h)$ and satisfy the surface and the bottom boundary conditions. With the "stretched" vertical coordinate this will have the form:

$$
\begin{array}{ll}
\Delta_{3} \mathrm{G}=4 \pi \delta(\mathrm{r}, \tilde{\mathrm{z}}-\mathrm{h}) & 0 \leq \tilde{\mathrm{z}} \leq 1 \\
\mathrm{G}+\mathrm{S}_{0} \mathrm{G}_{\tilde{\mathrm{z}}}=0 & \text { @ } \tilde{\mathrm{z}}=0 \\
\mathrm{G}+\mathrm{S}_{1} \mathrm{G}_{\tilde{\mathrm{z}}}=0 & \text { @ } \tilde{\mathrm{z}}=1
\end{array}
$$

It is convenient to split the Green's function into two parts, one corresponding to an interior potential vorticity anomaly and another, "boundary" part responsible for satisfying the boundary conditions :

$$
\mathrm{G}=\mathrm{G}^{\mathrm{int}}+\mathrm{G}^{\text {bound }}
$$

The interior part will be subject to

$$
\begin{array}{ll}
\Delta_{3} \mathrm{G}^{\text {int }}=4 \pi \delta(\mathrm{r}, \tilde{\mathrm{z}}-\mathrm{h}) & 0 \leq \tilde{\mathrm{z}} \leq 1 \\
\mathrm{G}^{\text {int }} \rightarrow 0 & \text { @ r, }|\tilde{\mathrm{z}}| \rightarrow \infty
\end{array}
$$

The "boundary" part then must be a solution of a following problem:

$$
\begin{array}{cc}
\Delta_{3} G^{\text {bound }}=0 & 0 \leq \tilde{\mathrm{z}} \leq 1 \\
\mathrm{G}^{\text {bound }}+\mathrm{S}_{0} \mathrm{G}_{\tilde{\mathrm{z}}}^{\text {bound }}=-\left(\mathrm{G}^{\text {int }}+\mathrm{S}_{0} \mathrm{G}_{\tilde{\mathrm{z}}}^{\text {int }}\right) & \text { @ } \tilde{\mathrm{z}}=0
\end{array}
$$




$$
\mathrm{G}^{\text {bound }}+\mathrm{S}_{1} \mathrm{G}_{\tilde{\mathrm{z}}}^{\text {bound }}=-\left(\mathrm{G}^{\text {int }}+\mathrm{S}_{1} \mathrm{G}_{\tilde{\mathrm{z}}}^{\text {int }}\right) \quad \quad @ \quad \tilde{\mathrm{z}}=1
$$

Solution for the "interior" part is simple and equivalent to a field of electrostatic (or gravitational) charge placed at the point $(0,0, \mathrm{~h})$ :

$$
\mathrm{G}^{\mathrm{int}}=-\frac{1}{\mathrm{R}}
$$

Here and in the reminder of the text we denote by capital $R$ a three-dimensional distance and by small $\mathrm{r}$ - horizontal distance. Particularly in this case

$$
\mathrm{R}^{2}=\mathrm{r}^{2}+(\tilde{\mathrm{z}}-\mathrm{h})^{2}, \quad \mathrm{r}^{2}=\mathrm{x}^{2}+\mathrm{y}^{2}
$$

Using (A1.21) the right-hand side of (A1.20) will become:

$$
\begin{aligned}
& \Phi^{0}=-\left.\left(G^{\text {int }}+S_{0} G_{\tilde{z}}^{\text {int }}\right)\right|_{\tilde{z}=0}=\frac{1}{\sqrt{r^{2}+h^{2}}}+\frac{S_{0} h}{\sqrt{r^{2}+h^{2}}} \\
& \Phi^{1}=-\left.\left(G^{\text {int }}+S_{1} G_{\tilde{z}}^{\text {int }}\right)\right|_{\tilde{z}=1}=\frac{1}{\sqrt{r^{2}+(1-h)^{2}}}-\frac{S_{1}(1-h)}{\sqrt{r^{2}+(1-h)^{2}}}
\end{aligned}
$$

Solution for the "boundary" part as well as for "interior" one will be radially symmetric and we shall seek it in the form of a Bessel integral in the radial structure. The vertical dependence will have such a form that each harmonics will obey the interior equation (zero laplacian):

$$
\mathrm{G}^{\text {bound }}=\int_{0}^{\infty} \kappa\left[\mathrm{A}_{\kappa} \cosh (\kappa \tilde{\mathrm{z}})+\mathrm{B}_{\kappa} \sinh (\kappa \tilde{\mathrm{z}})\right] \mathrm{J}_{0}(\kappa r) d \kappa
$$

Then

$$
\mathrm{G}_{\mathrm{z}}^{\text {bound }}=\int_{0}^{\infty} \kappa^{2}\left[\mathrm{~A}_{\kappa} \sinh (\kappa \tilde{\mathrm{z}})+\mathrm{B}_{\kappa} \cosh (\kappa \tilde{\mathrm{z}})\right] \mathrm{J}_{0}(\kappa r) d \kappa
$$

where $\mathrm{J}_{0}$ - a zero-order Bessel function.

Now the left-hand side of the boundary conditions (A1.20b,c) will become: 


$$
\begin{array}{r}
\left(\mathrm{G}^{\text {bound }}+\mathrm{S}_{0} \mathrm{G}_{\mathrm{z}}^{\text {bound }}\right)=\int_{0}^{\infty} \kappa\left(\mathrm{A}_{\kappa}+\mathrm{B}_{\kappa} \mathrm{S}_{0} \kappa\right) \mathrm{J}_{0}(\kappa \mathrm{r}) d \kappa \\
\left(\mathrm{G}^{\text {int }}+\mathrm{S}_{1} \mathrm{G}_{\mathrm{z}}^{\text {int }}\right)=\int_{0}^{\infty} \kappa\left(\mathrm{A}_{\kappa}\left[\left(\cosh (\kappa)+\mathrm{S}_{1} \kappa \sinh (\kappa)\right]+\mathrm{B}_{\kappa}\left[\sinh (\kappa)+\mathrm{S}_{1} \kappa \cosh (\kappa)\right]\right) \mathrm{J}_{0}(\kappa \mathrm{r}) d \kappa\right. \\
@ \quad \tilde{\mathrm{z}}=1
\end{array}
$$

We shall expand also the right-hand side of $(\mathrm{A} 1.20 \mathrm{~b}, \mathrm{c})$ into a similar Bessel integral form:

$$
\begin{array}{lll}
\mathrm{G}^{\mathrm{int}}=-\int_{0}^{\infty} \kappa \mathrm{P}_{\kappa}^{0} \mathrm{~J}_{0}(\kappa r) d \kappa, & \mathrm{G}_{\tilde{\mathrm{z}}}^{\mathrm{int}}=-\int_{0}^{\infty} \kappa \mathrm{Q}_{\kappa}^{0} \mathrm{~J}_{0}(\kappa r) d \kappa & \text { @ } \tilde{\mathrm{z}}=0 \\
\mathrm{G}^{\text {int }}=-\int_{0}^{\infty} \kappa \mathrm{P}_{\kappa}^{1} \mathrm{~J}_{0}(\kappa r) d \kappa, & \mathrm{G}_{\tilde{\mathrm{z}}}^{\mathrm{int}}=\int_{0}^{\infty} \kappa \mathrm{Q}_{\kappa}^{1} \mathrm{~J}_{0}(\kappa r) d \kappa & @ \tilde{\mathrm{z}}=1
\end{array}
$$

where the Fourier (Bessel) coefficients $\quad \mathrm{P}_{\kappa}^{0}, \mathrm{P}_{\kappa}^{1}, \mathrm{Q}_{\kappa}^{0}, \mathrm{Q}_{\mathrm{K}}^{1} \quad$ are

$$
\begin{array}{ll}
\mathrm{P}_{\kappa}^{0}=\int_{0}^{\infty} \frac{\mathrm{r}}{\sqrt{\mathrm{r}^{2}+\mathrm{h}^{2}}} \mathrm{~J}_{0}(\mathrm{\kappa r}) d \mathrm{r} & \mathrm{P}_{\kappa}^{1}=\int_{0}^{\infty} \frac{\mathrm{r}}{\sqrt{\mathrm{r}^{2}+(1-\mathrm{h})^{2}}} \mathrm{~J}_{0}(\kappa \mathrm{r}) d \mathrm{r} \\
\mathrm{Q}_{\kappa}=\int_{0}^{\infty} \frac{\mathrm{rh}}{\sqrt{\mathrm{r}^{2}+\mathrm{h}^{2}} 3} \mathrm{~J}_{0}(\kappa \mathrm{r}) d \mathrm{r} & \mathrm{Q}_{\mathrm{\kappa}}^{1}=\int_{0}^{\infty} \frac{\mathrm{r}(1-\mathrm{h})}{\sqrt{\mathrm{r}^{2}+(1-\mathrm{h})^{2}} 3} \mathrm{~J}_{0}(\kappa \mathrm{r}) d \mathrm{r}
\end{array}
$$

This expansion procedure uses the self-inverting property of the two consecutive Bessel transforms:

$$
\mathrm{f}(\mathrm{r})=\int_{0}^{\infty} \hat{\mathrm{f}}_{\kappa} \mathrm{J}_{0}(\kappa \mathrm{r}) \kappa d \kappa, \quad \text { where } \quad \hat{\mathrm{f}}_{\kappa}=\int_{0}^{\infty} \mathrm{f}(\mathrm{r}) \mathrm{J}_{0}(\kappa \mathrm{r}) \mathrm{r} d \mathrm{r}
$$


(Note that in comparison with the cartesian Fourier transform this identity is satisfied without factor $1 / 2 \pi)$.

Comparing left-hand and right-hand side of (A1.20b,c) we shall obtain an expression for unknown Fourier (Bessel) coefficients $A_{\kappa}$ and $B_{\kappa}$ :

$$
\begin{aligned}
& A_{\kappa}=\frac{1}{D}\left(F_{\kappa}^{0}\left(\sinh (\kappa)+S_{1} \cosh (\kappa)\right)-F_{\kappa}^{1} S_{0}\right) \\
& B_{\kappa}=\frac{1}{D}\left(-F_{\kappa}^{0}(\cosh (\kappa)+\sinh (\kappa))+F_{\kappa}^{1}\right)
\end{aligned}
$$

where

$$
D=\left(1-S_{0} S_{1} \kappa^{2}\right) \sinh (\kappa)+\left(S_{1}-S_{0}\right) \cosh (\kappa)
$$

and Bessel coefficients $\mathrm{F}_{\mathrm{K}}^{0}, \mathrm{~F}_{\mathrm{K}}^{1}$ are:

$$
\begin{aligned}
& \mathrm{F}_{\kappa}^{0}=\int_{0}^{\infty} \Phi^{0} \mathrm{~J}_{0}(\kappa r) \kappa d \kappa=\mathrm{P}_{\kappa}^{0}+\frac{1}{\mathrm{~m}_{0}} \mathrm{Q}_{\kappa}^{0} \\
& \mathrm{~F}_{\kappa}^{1}=\int_{0}^{\infty} \Phi^{1} \mathrm{~J}_{0}(\kappa r) \kappa d \kappa=\mathrm{P}_{\kappa}^{1}-\frac{\mathrm{m}_{0}}{1-\mathrm{m}_{0}} Q_{\kappa}^{1}
\end{aligned}
$$

Finally, expressing constants $S_{0}$ and $S_{1}$ through the parameter $m_{0}$ we obtain that the Green's function will have the following form:

$$
\begin{aligned}
& \mathrm{G}(\mathrm{r}, \tilde{\mathrm{z}}, \mathrm{h})=-\frac{1}{\mathrm{R}}+\int_{0}^{\infty} \kappa\left[\mathrm{A}_{\kappa} \cosh (\kappa \tilde{\mathrm{z}})+\mathrm{B}_{\kappa} \sinh (\kappa \tilde{\mathrm{z}})\right] \mathrm{J}_{0}(\kappa \mathrm{r}) d \kappa \\
& \mathrm{A}_{\kappa}=\frac{1}{\mathrm{D}}\left(\mathrm{F}_{\kappa}^{0}\left(\sinh (\kappa)+\frac{1-\mathrm{m}_{0}}{\mathrm{~m}_{0}} \cosh (\kappa)\right)-\mathrm{F}_{\kappa}^{1} \frac{1}{\mathrm{~m}_{0}}\right) \\
& \mathrm{B}_{\kappa}=\frac{1}{\mathrm{D}}\left(-\mathrm{F}_{\kappa}^{0}(\cosh (\kappa)+\sinh (\kappa))+\mathrm{F}_{\kappa}^{1}\right)
\end{aligned}
$$

where

$$
\mathrm{D}=\left(1-\frac{1-\mathrm{m}_{0}}{\mathrm{~m}_{0}^{2}} \kappa^{2}\right) \sinh (\kappa)+\cosh (\kappa)
$$


and functions $\mathrm{F}_{\mathrm{K}}^{0}$ and $\mathrm{F}_{\mathrm{K}}^{1}$ are given by expressions (A1.27).

Summarizing, we recapitulate the steps needed for computation of the Green's function: first, we must calculate the Bessel coefficients $\mathrm{P}_{\mathrm{K}}^{0}, \mathrm{P}_{\mathrm{K}}^{1}, \mathrm{Q}_{\mathrm{K}}^{0}, \mathrm{Q}_{\mathrm{K}}^{1}$ using (A1.26) and, hence, $\mathrm{F}_{\mathrm{K}}^{0}$ and $\mathrm{F}_{\mathrm{K}}^{1}$, using (A1.27), substitute it into (A1.28b,c) and, finally, perform the inverse Bessel transform (A1.28a). Our final goal is not the Green's function itself but the azimuthal velocity profile. It can be computed as a radial derivative of the Green's function:

$$
\mathrm{v}=\frac{\partial \mathrm{G}}{\partial \mathrm{r}}
$$

Azimuthal velocity is a function of radial and vertical coordinates and position of a PV anomaly: $v=v(r, \tilde{z}, h)$. Examples of the azimuthal velocity dependence on $r$ are shown in figure

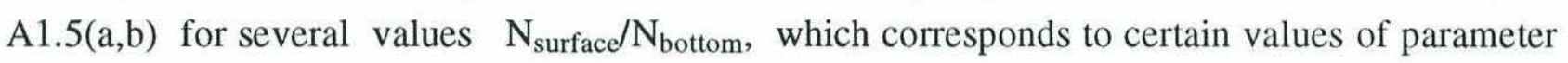
$\mathrm{m}_{0}$ (and $\lambda=0$ ). Altogether four cases are given: velocity profiles at the surface and the bottom for a PV anomaly placed near the bottom at $\mathrm{h}=0.1$ (fig. A1.5a) and at $\mathrm{h}=0.75$ (fig. A1.5b). One can see from these pictures that even for very non-uniform stratification (a large ratio of the surface to bottom buoyancy frequency) radial profiles are quite similar to the case of constant $\mathrm{N}$. When a PV anomaly is near the bottom the velocity at the bottom (where $\mathrm{N}(\mathrm{z})$ is minimal) is smaller than for constant $\mathrm{N}$ (when $\mathrm{N}_{\text {surface }} / \mathrm{N}_{\text {bottom }}=30$ the maximum radial velocity is about 2 times smaller than for the uniform stratification); at the surface the velocity is slightly larger for positive $m_{0}$. When a PV anomaly is near the surface the velocities are larger everywhere, but near the bottom the increase is very small while at the surface it is more substantial and can reach several times its value for $\mathrm{N}=$ const. Most of the difference for various stratification profiles is local, for $\mathrm{r}<1$, that is for horizontal distances less than the depth $\widetilde{\mathrm{H}}$ in stretched coordinates. For $\mathrm{r}>1$ stratification practically does not make any difference as barotropic behavior $(\sim 1 / \mathrm{r})$ takes over.

Using a set of such profiles for different parameters $m_{0}$ (and, generally, $\lambda$ ) and different depths $\mathrm{h}$ and $\tilde{\mathrm{z}}$ one can in principle work out a parameterization formula - an explicit analytical relation for behavior of azimuthal velocity $\mathrm{v}\left(\mathrm{r}, \mathrm{h}, \tilde{\mathrm{z}}, \mathrm{m}_{0}, \lambda\right)$ which would simulate all these profiles with needed accuracy. We would not consider this issue here in details, though we used a rather simple parameterization of velocity for the vortex - bottom topography interaction problem in chapter 6 . 

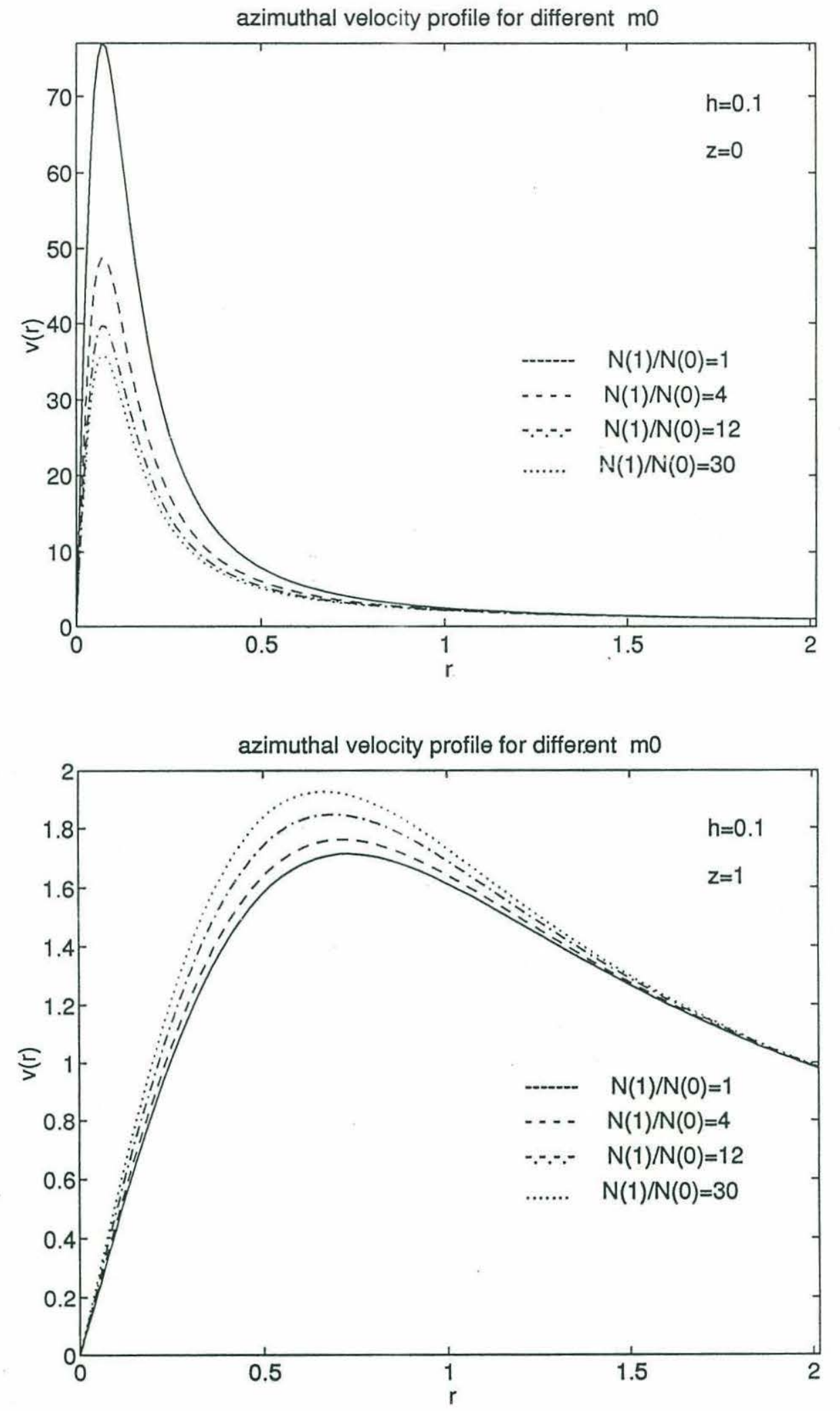

Figure A1.5 (a). Examples of azimuthal velocity profiles for near-bottom PV anomaly. 

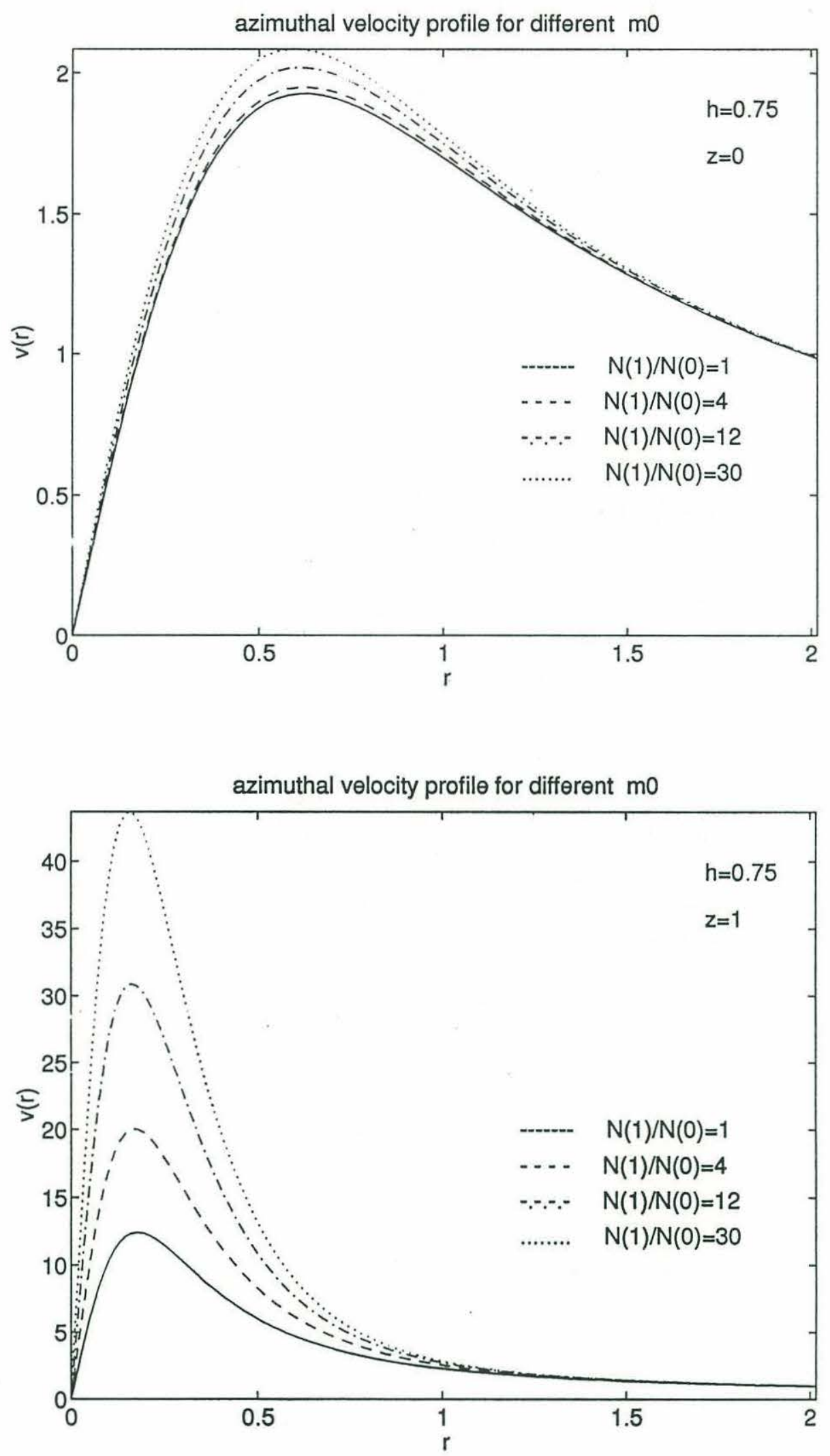

Figure A1.5 (b). Examples of azimuthal velocity profiles for near-surface PV anomaly. 


\section{Appendix 2}

\section{Some details of the numerical}

\section{implementation of the semi-}

\section{lagrangian contour dynamics method}

As I already mentioned in chapter 4 at each timestep in the model one need to perform the following procedures: interpolation of density at the bottom from the Lagrangian moving contours onto the Eulerian regular grid to obtain density and hence vorticity anomalies, inversion of the vorticity field at the bottom to obtain velocities at the same grid and interpolation of velocities back onto moving contours.

In the present case the treatment of moving contours was simplified: their cross-slope horizontal coordinate $\mathrm{y}$ was allowed to be only a univalued function of along-slope coordinate $\mathrm{x}$, so each contour had only one intersection with any "vertical" (cross-slope) line $\mathrm{x}=$ const. And I actually followed the $y$-coordinates of such intersection as dynamical variables of our model. I tried two methods to do this: reinterpolation of moving points on the vertical lines at each time step, and computing the $\mathrm{x}$-derivative of the contour displacement using high-order finite difference. Both techniques gave very similar results, and I present here only calculations with reinterpolation procedure. Therefore such setting could not actually describe the "wave-breaking" process, when contour can have multiple intersections with $\mathrm{x}=$ const lines, but we could monitor the conditions when this breaking is likely. Until some portions of the contours became aligned almost in the crossslope direction this procedure is accurate enough.

In this case the interpolation is reduced to a one-dimensional procedure. At each timestep I have a matrix $\mathrm{Y}_{\mathrm{i}, \mathrm{j}}$ of $\mathrm{y}$-coordinates where moving contours intersect the "vertical" lines $\mathrm{x}=\mathrm{x}_{\mathrm{j}}$, $\mathrm{j}=1 \ldots \mathrm{K}_{\mathrm{h}}$ is the total number of points in each contour, $\mathrm{i}=1 \ldots \mathrm{K}_{\mathrm{c}}$ is the total number of contours. We also have values of the function $\eta_{i}$ (density) at each contour. We interpolate this function along 
the "vertical" lines from the contours whose intersections are nearest to the given gridpoint. We use the 4-th order (5-point) Lagrangian polynomials:

$$
\eta_{\text {int }}=\sum_{\mathrm{i}=1}^{5} \eta_{\mathrm{i}}\left(\prod_{\mathrm{k}=1, \mathrm{k} \neq \mathrm{i}}^{5} \frac{\left(\mathrm{Y}_{\mathrm{int}}-\mathrm{Y}_{\mathrm{i}}\right)}{\left(\mathrm{Y}_{\mathrm{k}}-\mathrm{Y}_{\mathrm{i}}\right)}\right)
$$

Here the subscript "int" denotes the interpolated values and coordinates, and indices $i$ and $\mathrm{k}$ stand for summation and product over the nearest points. The interpolation of velocities back to the moving contours is made in a similar way.

The inversion from the regular grid is performed by a convolution with a corresponding Green's function (we used the Green's functions directly for velocities instead of the streamfunction). The velocity field at the gridpoints can be written as following:

$$
\begin{aligned}
\mathrm{u}_{\mathrm{i}, \mathrm{j}} & =\sum_{\mathrm{m}=0}^{\mathrm{m}_{0}} \sum_{\mathrm{k}=1}^{\mathrm{K}_{\mathrm{c}}} \widetilde{\mathrm{U}}_{\mathrm{i}, \mathrm{k}}^{\mathrm{m}}\left(\phi_{\mathrm{k}, \mathrm{j}+\mathrm{m}}+\phi_{\mathrm{k}, \mathrm{j}-\mathrm{m}}\right) \\
\mathrm{v}_{\mathrm{i}, \mathrm{j}} & =\sum_{\mathrm{m}=0}^{\mathrm{m}_{0}} \sum_{\mathrm{k}=1}^{\mathrm{K}_{\mathrm{c}}} \widetilde{\mathrm{v}}_{\mathrm{i}, \mathrm{k}}^{\mathrm{m}}\left(\phi_{\mathrm{k}, \mathrm{j}+\mathrm{m}}-\phi_{\mathrm{k}, \mathrm{j}-\mathrm{m}}\right)
\end{aligned}
$$

where Green's function matrices $\widetilde{U}_{\mathrm{i}, \mathrm{k}}^{\mathrm{m}}$ and $\widetilde{\mathrm{V}}_{\mathrm{i}, \mathrm{k}}^{\mathrm{m}}$ describe the velocities in the $\mathrm{j}$-th column of velocity matrix $v_{i, j}$ induced by density anomalies $\phi_{j+m}$ and $\phi_{j-m}$ at the colums with numbers $\mathrm{j}+\mathrm{m}$ and $\mathrm{j}-\mathrm{m}$ (and $\mathrm{k}$ is a summation index). By a "column" with number $\mathrm{j}$ I mean the vector of gridded values along the line $\mathrm{x}=\mathrm{x}_{\mathrm{j}}$. This summation is made directly for neighbouring colums up to the distance $m_{0}$ between them. For the colums which are separated further we appoximate the matrices $\widetilde{U}_{\mathrm{i}, \mathrm{k}}^{\mathrm{m}}$ and $\widetilde{\mathrm{V}}_{\mathrm{i}, \mathrm{k}}^{\mathrm{m}}$ by a sum of "basis" matrices $\widetilde{U}_{\mathrm{i}, \mathrm{k}}^{m_{b}(\mathrm{n})}$ and $\widetilde{\mathrm{V}}_{\mathrm{i}, \mathrm{k}}^{\mathrm{m}_{\mathrm{b}}(\mathrm{n})} \quad \mathrm{n}=1 . . \mathrm{n}_{\mathrm{b}}$. In the matrix form this will look like

$$
\mathbf{u}=\sum_{\mathrm{n}=1}^{\mathrm{n}_{\mathrm{b}}} \widetilde{\mathbf{U}}^{\mathrm{m}_{\mathrm{b}}(\mathrm{n})} \cdot \Phi \cdot \mathbf{C}_{\mathrm{u}}^{\mathrm{n}}, \quad \mathbf{v}=\sum_{\mathrm{n}=1}^{\mathrm{n}_{\mathrm{b}}} \widetilde{\mathbf{V}}^{\mathrm{m}_{\mathrm{b}}(\mathrm{n})} \cdot \Phi \cdot \mathbf{C}_{\mathrm{v}}^{\mathrm{n}}
$$

where the matrices $\mathbf{C}_{\mathrm{u}}^{\mathrm{n}}$ and $\mathbf{C}_{\mathrm{v}}^{\mathrm{n}}$ consist of corresponding interpolation coefficients. In the presented case the number of "neighbouring" columns is $\mathrm{m}_{0}=8$ and the number of "basis" matrices $\mathrm{n}_{\mathrm{b}}=5$.

The interpolation coefficients are computed with the "basis" functions $b_{n}(x)=\left(x^{2}+a_{n}^{2}\right)^{-1 / 2}$ instead of usual polynomials to represent the asymptotic structure of the velocity field (this is a very 
accurate procedure for our velocity structure, while the polynomial interpolation fails miserably in this case).

This way of inversion is certainly easier than the direct summation over each pair of points. It is easily and elegantly programmable in the matrix form and consists of only about a dozen lines of the MATLAB code. But still it does not fully utilize the potential of Green's functions.

It is interesting to note that inversion via Green's functions has not received much attention in numerical methods (contour dynamics is a rare exception) because of several reasons. It is conceptually more difficult than obvious finite-differencing, it is often not clear how to accommodate the boundary conditions for a domain with a complicated shape, and it was usually considered expensive numerically because in its primitive form it has quadratic cost in number of points. But using more sophisticated tricks, like the moment acceleration, its cost can be reduced to the order $\mathrm{N} \log \mathrm{N}$ instead of $\mathrm{N}^{2}$. A problem with boundary conditions can usually also be addressed and resolved. And these factors, combined with a superior accuracy, make this type of inversion procedure very promising. I have not implemented these ideas in the current version of the model but work is currently under way to use them to construct a much more efficient inversion procedure.

In addition to these main procedures of interpolation and inversion I perform some auxillary operations, mentioned in chapters 4 and 5. Some "smoothing" of contour lines is made which approximately corresponds to the ordinary viscosity. So at each time step the positions $\mathrm{Y}_{\mathrm{i}, \mathrm{j}}$ of contours intersections with "vertical" lines are corrected as follows:

$$
Y_{i, j}=Y_{i, j}+v_{h}\left(Y_{i, j-1}+Y_{i, j+1}-2 Y_{i, j}\right)+v_{v}\left(Y_{i-1, j}+Y_{i+1, j}-2 Y_{i, j}\right)
$$

where the viscosity coefficients $v_{\mathrm{h}}$ and $v_{\mathrm{v}}$ are small enough to ensure that dissipation is not important. The characteristic relaxation time due to this viscosity at our typical scales of motion is more than 20 units (periods of short topographic waves). Also to prevent topographic waves from reflecting back from the left end of the domain we relax their intersections $\mathrm{Y}_{\mathrm{i}, \mathrm{j}}$ towards the unperturbed positions in the "sponge" layer at the left side:

$$
Y_{i, j}=Y_{i, j}^{0}+\left(Y_{i, j}-Y_{i, j}^{0}\right) \cdot S_{i, j}
$$

where $\mathrm{S}_{\mathrm{i}, \mathrm{j}}$ is a "sponge matrix" which structure is shown in figure 5.3.

I also implement a "moving grid" technique to study steady motion of vortices to reduce the influence of the domain boundaries. To make this we set the "permissible" area within our computational domain bounded by some left, right, lower and upper coordinates: $\mathrm{x}_{\mathrm{l}}, \mathrm{x}_{\mathrm{r}}, \mathrm{y}_{\mathrm{l}}, \mathrm{y}_{\mathrm{u}}$ (in our calculations the "permissible" area was only about a quarter of the whole domain). Then in the course of calculations we do the following: when a vortex moves out of the "permissible" 
domain all density contours and the trajectory of the vortex itself are shifted one grid scale in the direction of vortex motion. The last contour behind a vortex is discarded and the first contour ahead of it is extrapolated (with some smoothing) from several neighboring ones. This process is repeated many times during a given run as the vortex tends to move out of the "permissible" domain.

To check the accuracy of the numerical procedures I looked for propagation of linear topographic waves as it can be represented in the model. I initialized the model with a packet of small-amplitude waves of a sinusoidal form (with an along-slope wavenumber) with a gaussian envelope. For very short waves such a packet is nearly monochromatic and should propagate through the domain without significant change of its shape with a constant group velocity which is given by the expression:

$$
c_{\mathrm{g}}=\frac{\mathrm{d} \omega}{\mathrm{dk}}=-\mathrm{N} \alpha(\mathrm{k} \tanh (\mathrm{k} \widetilde{\mathrm{H}}))^{-1}(1 / \mathrm{k} \widetilde{\mathrm{H}}+2 / \sinh (2 \mathrm{k} \widetilde{\mathrm{H}}))
$$

Here $\mathrm{k}$ is the along-slope wavenumber, $\omega$ is the frequency, $\widetilde{\mathrm{H}}$ is a "stretched" depth, $\alpha$ is the slope angle, $\mathrm{N}$ is the buoyancy frequency.

I performed several tests for the same domain but for different wavenumbers $k$ of the sinusoidal wave. One example is shown in the figure A2.1 for initial and final states. As usual the contour displacements and density anomalies are given. One can see that indeed the wave packet does not change its shape significantly and as a whole propagates slowly to the left of the upslope direction.

I estimated the group velocity in these tests as the along-slope displacements of the centroid of the wave amplitude (in this case the absolute value of the density anomaly) divided by the elapsed time. The results of these tests are presented in figure A2.2 where these estimates are compared with the theoretical curve computed using (A2.1). The errorbars in this figure were estimated as a relative difference between the computed initial position of the centroid and the theoretical center (maximum) of density anomaly which we put into the model. This figure shows a sensible behavior of the model group velocity depending on the wavenumber. The difference between the computed estimates and the theoretical curve is within the errorbars of the test. Any discrepancy includes combined effects of the waves dispersion (because of the non-monochromaticity), finiteness of the domain and dissipation. Considering these factors the model is good enough in representing the dynamics of linear waves.

Another example which we present here in figure A2.3 is the dispersion of the initial dipolar perturbation, similar to the one induced by the vortex in our previous calculations. One can see that the perturbation propagates to the left with a significant dispersion and after several short waves periods loses it shape completely. 
The whole program was written entirely in the MATLAB code which allows to put most of the operations in the short and convenient matrix form without writing long and incomprehensible cycles. The MATLAB is also very convenient to handle the output of the calculations and to present the results in the graphic form. 

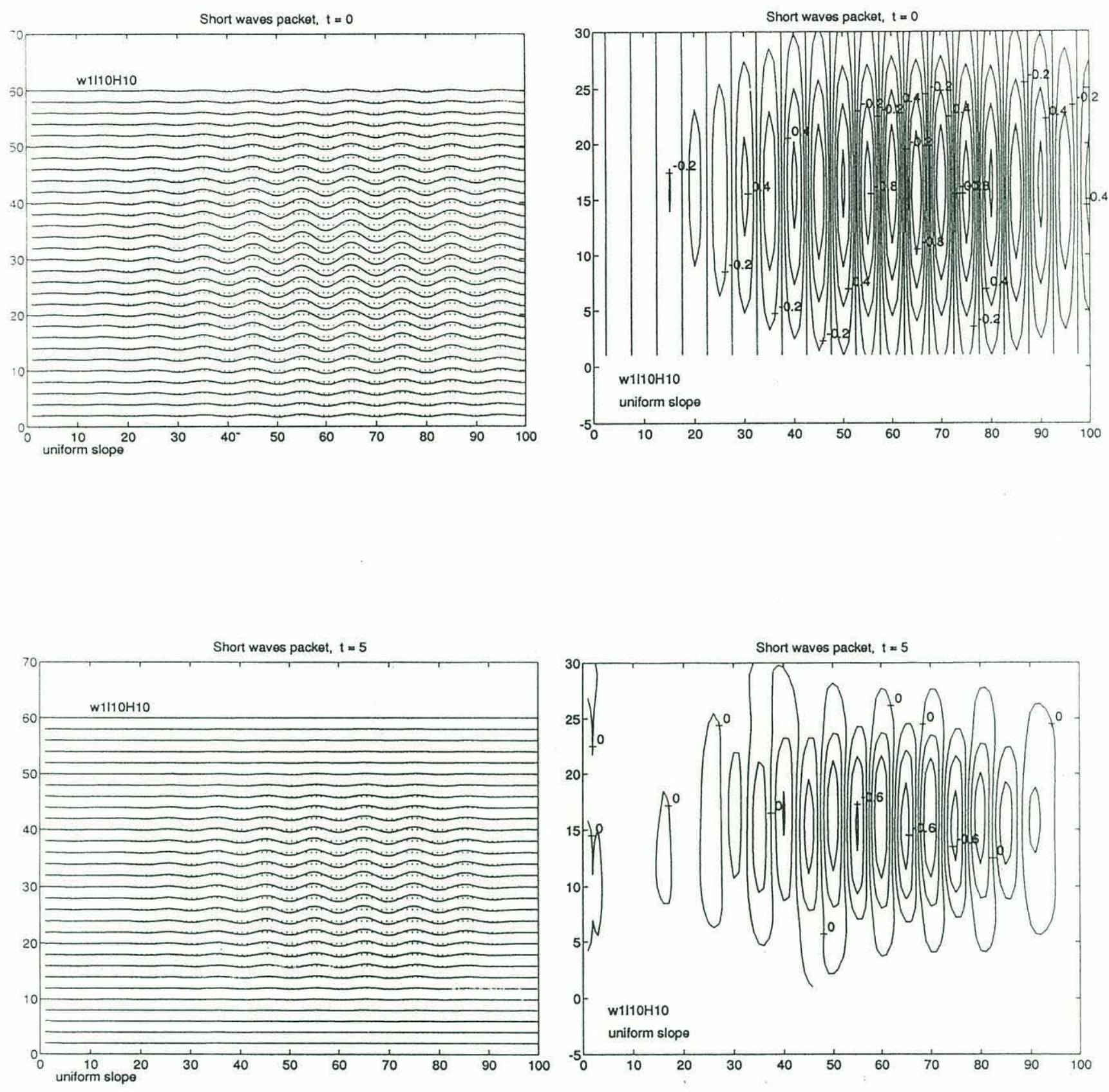

Figure A2.1. Evolution of the short wave packet. 
Test of the group velocity of a wave packet

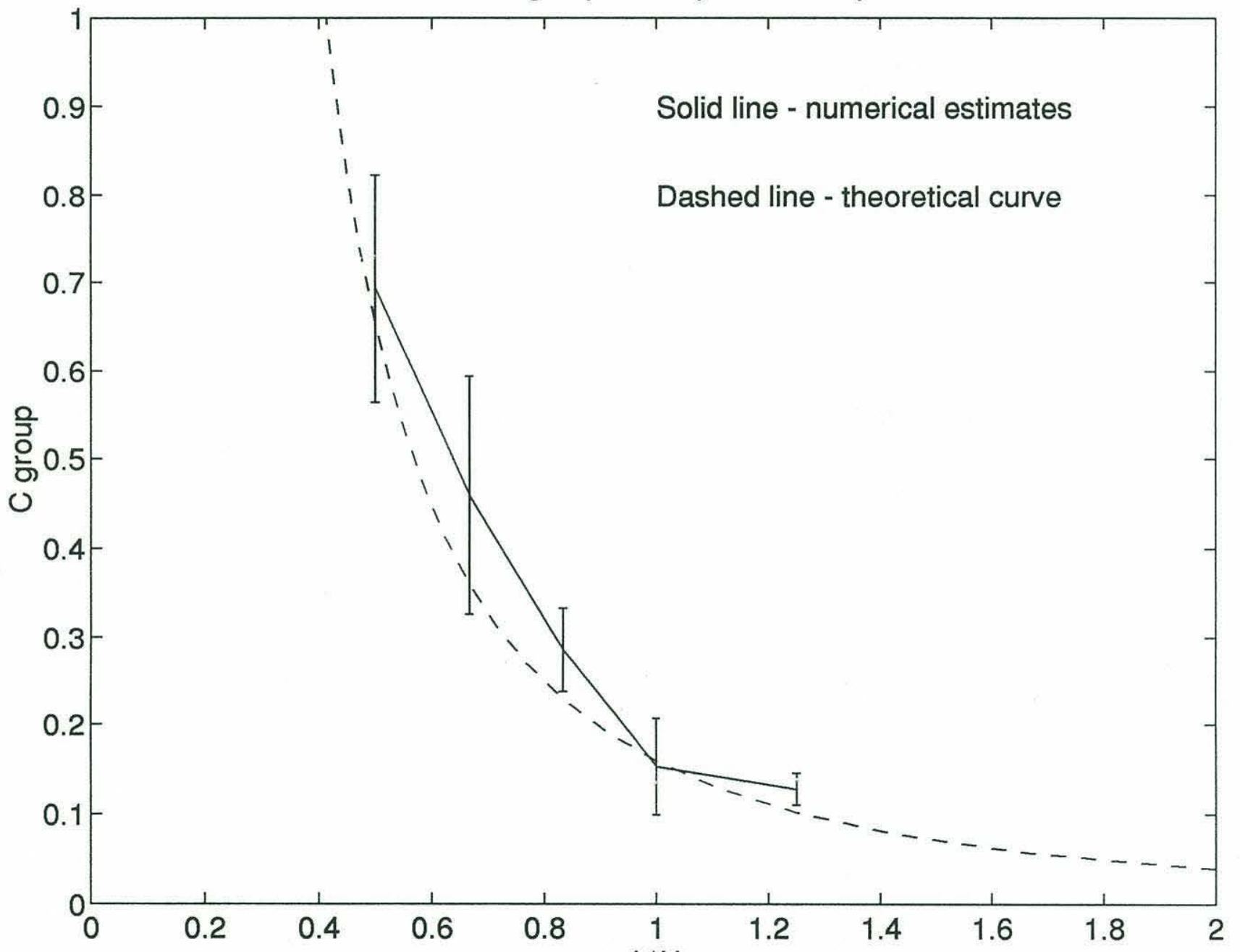

Figure A2.2 Comparison of the theoreticat athd computed group speeds of short wave packets. 

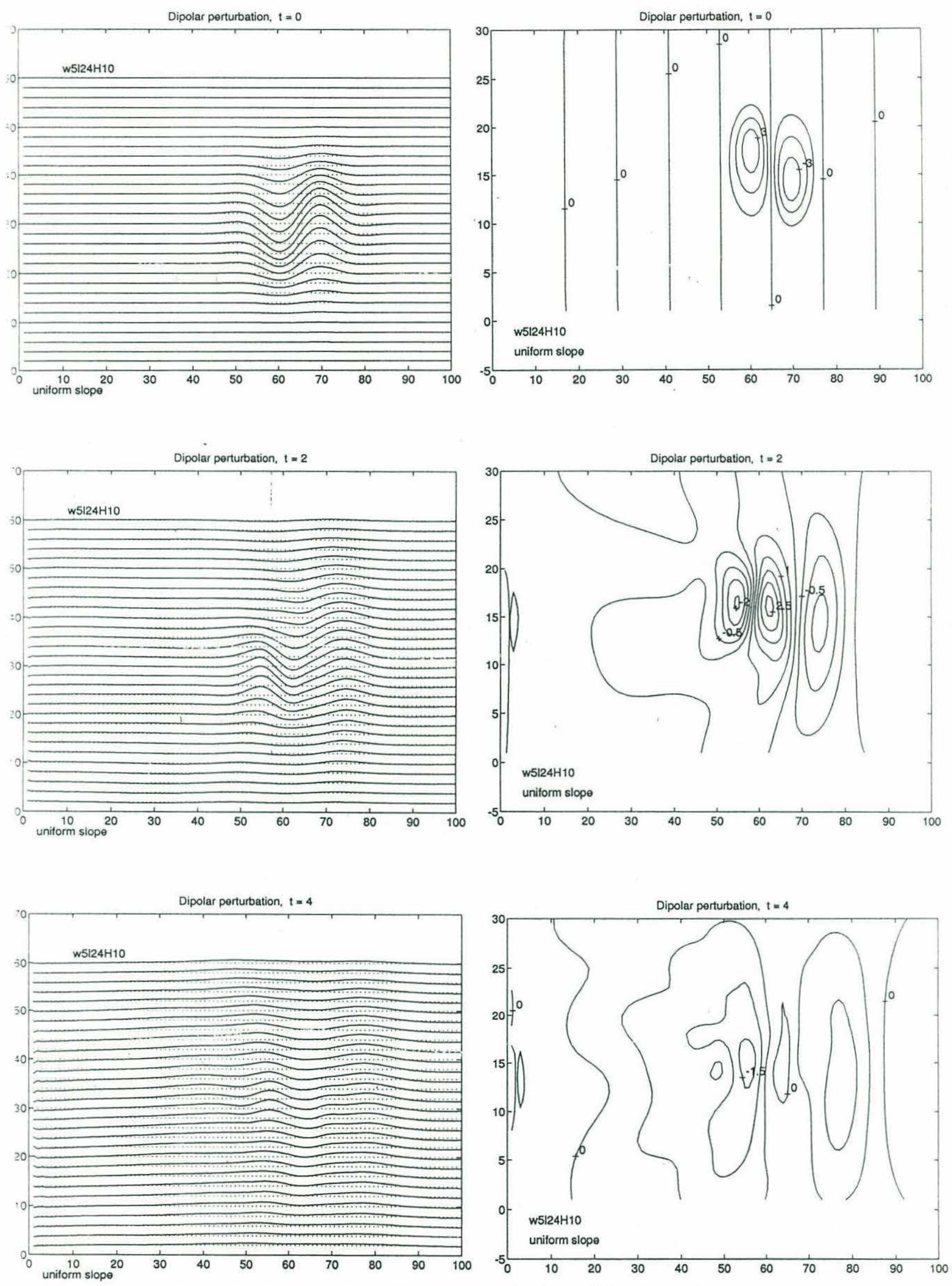

Figure A2.1 Evolution of the dipolar perturbation without a vortex. 


\section{References}

Bleck R. \& L. T. Smith, 1990. A wind-driven isopycnic coordinates model of the North and equatorial Atlantic ocean. 1. Model development and supporting experiments. J. Geophys. Res., V. 95, \# C3, 3273-3285.

Bogomolov V. A., 1985. Motion of a vortex on a rotating sphere. Izv. Akad. Nauk. SSSR, Fiz. Atmos. i Okeana. V.21, \#4, 391-396.

Brenner S., 1989. Structure and evolution of warm-core eddies in the eastern Mediterranian Levantine Basin. J. Geophys. Res., 94 (C9), 12593-12602.

Bretherton F. P., 1966. Critical layer instability in baroclinic flows. Quart. J. Royal Meteor. Soc., V. 92, 325-334.

Bretherton F. P. \& Haidvogel. D. E., 1976. Two-dimensional geostrophic turbulence above topogarphy. J. Fluid Mech., V. 78, 129-154.

Carnevale G. F., R. C. Kloostersiel \& G. J. F. van-Heijst, 1991. Propagation of barotropic vortices over topography in a rotating tank. J. Fluid Mech., V. 233, 119-140.

Carnevale G. F. \& Frederiksen J. S., 1987. Nonlinear stability and statistical mechanics of a flow over topography. J. Fluid Mech., V. 175, 157-181.

Chapman D. C. \& K. H. Brink, 1987. Shelf and slope circulation induced by fluctuating offshore forcing. J. Geophys. Res., V. 92, 11741-11759.

Charney J. G., 1971. Geostropic turbulence. J. Atmos. Sc., V. 28, \#9, 1087-1095.

Cheney R. E., W. H. Gemmill, M. K. Shank, P. L. Richardson \& D. Webb, 1976. Tracking a Gulf Stream ring with SOFAR floats. J. Phys. Oceanography, V. 6, 741-749.

Cornillon P., R. Weyer \& G. Flierl, 1989. Translational velocity of warm core rings relative to the slope water. J. Phys. Oceanography, 19, \#9, 1317-1332.

Gryanic V. M., 1983. Dynamics of geostrophic vortices in a two-layer model of the atmosphere (or the ocean). Oceanology, V. 19, \#3, 171-179.

Evans R. H., K. S. Baker, O. B. Brown \& R. C. Smith, 1985. Chronology of warm-core ring 82B. J. Geophys. Res., V. 90, \# C5, 8803-8811.

Flierl G.R., 1977. The application of linear quasi-geostrophic dynamics to Gulf Stream rings. J. Phys. oceanogr., V.7, 365-379.

Flierl G.R., 1978. Models of vertical structure and the calibration of two layers models. Dyn. Atmos. \& Oceans, V.2, \#3, 341-381.

Flierl G. R., M. E. Stern \& J. A.Whitehead. The physical significance of modons: laboratory experiments and general physical constraints. Dyn. Atmos. Oceans, V.7, 233-263. 
Flierl G.R., 1984. Rossby wave radiation from a strongly nonlinear warm eddy. J. Phys. oceanogr., 14, \#1, 47-58.

Flierl G. R., 1987. Isolated eddy models in geophysics. Ann. Rev. Fluid Mech. V.19, 493530 .

Herring , 1977. On the statistical theory of two-dimensional topographic turbulence. J. Atmos. Sci., V. 34, 1731-1750.

Hogg N. G. \& H. M. Stommel, 1985. The heton, an elementary interaction between discrete baroclinic vortices and its implications concerning eddy heat flow. Proc. R. Soc. London Ser. A 397: 1-20.

Holloway G., 1992. Representing topographic stress for large-scale ocean models. J. Phys. oceanogr., V. 22, \#9, 1033-1046.

Ivanov A. F. \& A. N. Paramonov, 1980. Evolution of an individual cyclonic vortex. In "Synoptic eddies in the ocean", Kiev, Naukova dumka, 107-114.

Kelley E. A. \& G. L. Weatherly, 1985. Abyssal eddies near the Gulf Stream. J. Geophys. Res., 90, \#C2, 3151-3159.

Lewis J. K. \& A. D. Kirwan Jr., 1985. Some observations of ring-topography and ring-ring interactions in the gulf of Mexico. J. Geophys. Res., 90, \#C5, 9017-9028.

Louis J. P., B. D. Petrie \& P. D. Smith, 1982. Observation of topographic Rossby waves on the continental margin off Nova Scotia. J. Phys. Oceanography, V. 12, \#1, 47-55.

Louis J. P. \& P. D. Smith, 1982. The development of the barotropic radiation field of an eddy over a slope. J. Phys. Oceanography, V. 12, \#1, 56-73.

Malanotte-Rizzoli P. \& M. C. Hendershott, 1980. Solitary Rossby waves over random relief and their stability. Part 1: Analytical theory. Dyn. Atmos. Oceans, V. 4, \#4, 247-260.

McWilliams J. C. \& Flierl G. R., 1979. On the evolution of isolated, nonlinear vortices. J. Phys. Oceanography, V.9, 1155-1182.

Meacham S. P., K. K. Pankratov, A. F. Shchepetkin \& V. V. Zhmur, 1993. The interaction of ellipsoidal vortices with background shear flow in a stratified fluid. To appear in Dyn. Atmos. Oceans.

Mied R. P. \& Lindeman G. R., 1979. The propagation and evolution of cyclonic Gulf Stream rings. J. Phys. Oceanography, V.9, 1182-1206.

Nof D., 1983. The translation of isolated cold eddy on a sloping bottom. Deep Sea Res., 30, 171-182.

Swaters G. E. \& G. R. Flierl, 1991. Dynamics of ventillated coherent eddies on a sloping bottom. J. Fluid Mech, V. 223, 565-589.

Owens W. B. and F. P. Bretherton, 1978. A numerical study of mid-ocean mesoscale eddies. Deep Sea Res., V.25, \#1, 1-14. 
Pedlosky J., 1984. The equations of geostrophic motion in the ocean. J. Phys. Oceanography, V. $14,448-456$.

Pedlosky J., 1985. Instability of heton clouds. J. Atmos. Sci., 42, 1477-1486.

Pedlosky J., 1987. Geophysical Fluid dynamics. New York: Springer-Verlag. 2nd ed.

Prandtl L., 1952. The essentials of fluid dynamics. Blackie and sons, 3rd ed., $452 \mathrm{pp}$

Qiu B., 1990. Low-frequency shelf-slope responses induced by localized offshore forcing. $J$. Geophys. Res., V. 95, 9447-9459.

Reznik, 1991. Motion of a point vortex on the beta-plane. Oceanology, V. 30, \#5, 523-528. (English transl.).

Rhines P. B., 1970. Edge-, bottom-, and Rossby waves in a rotating stratified fluid. Geophys. Fluid Dynamics, 1, \#3, 273-302.

Rhines P. B., 1977. The dynamics of unsteady currents. The Sea, V. 6, 189-318.

Shaw P. T. \& S. Divakar, 1991. Generation of topographic waves over the continental margin. J. Phys. Oceanography, V. 21, 1032-1042.

Smith D. C., IV \& J. J. O'Brien, 1986. The interaction of a two-layer isolated mesoscale eddy with bottom topography. J. Phys. Oceanography, V. 13,\#9, 1681-1697.

Smith D. C., IV, 1986. A numerical study of Loop current eddy interaction with topography in the western Gulf of Mexico. J. Phys. Oceanography, V. 16, \#7, 1260-1272.

Sutyrin G. G., 1988. Motion of an intense vortex on a rotating sphere. Izv. Akad. Nauk SSSR, Mech. Zhid. i Gasa, \#2, 68-77.

Sutyrin G. G. \& G. R. Flierl, 1993. Intense vortex motion on the beta-plane. Part 1. Development of the beta-gyres. In press.

Treguier A. M. \& B. L. Hua, 1988. Influence of bottom topography on quasigeostrophic turbulence in the ocean. Geophys. Astrophys. Fluid Dyn., V. 43, 265-305.

Treguier A. M. \& J. C. McWilliams, 1990. Topographic influences on a wind-driven stratified flow in a beta-plane channel: an idealized model for Antarctic Circumpolar Current. J. Phys.

Oceanography, , V. 20, 321-343.

Veronis G., 1981. Dynamics of large-scale ocean circulation. In: Evolution of Physical Oceanography, ed. B. Warren, C. Wunsch, Cambridge, Mass: MIT Press, 140-183.

Walsh D. A model of a Mediterranean salt lens in external shear. Ph.D. Thesis. WHOI-92-21. Wang X., 1992. Interaction of an oceanic eddy with a straight-line topography. Ph.D. Thesis. WHOI-92.

Weatherly G. L. \& E. A. Kelly, 1982. "Too cold" bottom layers in HEBBLE area. J. Mar. Res, V. 40, 985-1012. 
Wolff J.-O. \& E. Maire-Rainer, 1991. Wind-driven flow over topography in a zonal $\beta$-plane channel: a quasigeostrophic model of the Antarctic Circumpolar current. J. Phys. Oceanography, V. 21, \# 2, 321-343.

Yano J.-I. \& G. R. Flierl, 1992. Isolated potential vorticity patches in quasi-geostrophic zonal flows. Dyn. Atmos. Oceans, V. 16, \#6, 439-472.

Zabusky N. J., M. N. Hughs \& K. V. Roberts, 1979. Contour dynamics for the Euler equations in two dimensions. J. Comp. Phys., V. 30, 96-106. 